\title{
Complex Nanomaterials in Catalysis for Chemically Significant Applications: From Synthesis and Hydrocarbon Processing to Renewable Energy Applications
}

\author{
Utkarsh Chadha ${ }^{D},{ }^{1}$ Senthil Kumaran Selvaraj ${ }^{D},{ }^{1}$ Hridya Ashokan (D), \\ Sai P. Hariharan $\mathbb{D}^{2},{ }^{2}$ V. Mathew Paul $\mathbb{D}^{2},{ }^{2}$ Vishal Venkatarangan $(\mathbb{D})$, \\ and Velmurugan Paramasivam (iD ${ }^{3}$ \\ ${ }^{1}$ Department of Manufacturing Engineering, School of Mechanical Engineering (SMEC), Vellore Institute of Technology (VIT), \\ Vellore 632014, Tamilnadu, India \\ ${ }^{2}$ School of Chemical Engineering (SCHEME), Vellore Institute of Technology (VIT), Vellore 632014, Tamilnadu, India \\ ${ }^{3}$ School of Mechanical and Automotive Engineering, College of Engineering and Technology, Dilla University, Dilla, \\ P.O. Box 419, Ethiopia
}

Correspondence should be addressed to Senthil Kumaran Selvaraj; senthilkumaran.s@vit.ac.in and Velmurugan Paramasivam; drvelmuruganp@du.edu.et

Received 15 November 2021; Revised 24 December 2021; Accepted 4 January 2022; Published 8 February 2022

Academic Editor: Ashutosh Sharma

Copyright (C) 2022 Utkarsh Chadha et al. This is an open access article distributed under the Creative Commons Attribution License, which permits unrestricted use, distribution, and reproduction in any medium, provided the original work is properly cited.

\begin{abstract}
The world is rapidly changing, the resources are getting depleted, and the demand for newer technologies and products is increasing. To keep up with these new advances, highly efficient catalytic routes need to be taken to be sustainable and ensure a drawn-out existence of resources for future generations. Catalysis turns out to be a significantly important field of application when it comes to the era of nanoscience, where all devices and technologies are becoming smaller and smaller in size with improved properties. When deeming the usability of a catalyst, it is of paramount importance to have a good understanding of their properties and their synergistic effect on the other reagents in the reaction. Over the last decade, the field of nanocatalysis has grown rapidly, both in homogeneous and heterogeneous catalysis. Given that nanoparticles have a high surface-to-volume ratio when compared to bulk materials, they are appealing as catalysts. Catalysts accelerate and boost thousands of different chemical reactions on a daily basis, forming the foundation of the multibillion-dollar chemical industry worldwide, a pathway leading to green chemistry, and a novel, yet crucial, environmental protection technology. As a result, in this review, the use of nanocatalysts and the application of their special features in the renewable energy, hydrocarbon processing, and fine chemical synthesis sector was explored. A detailed explanation of the working mechanism of these nanocatalysts, starting from how they are synthesized to the effect of modification of their surface, has been put together. We have tried to collect all the current progresses in these three sectors to the best of our abilities. Furthermore, it is anticipated that this paper would be useful for future researchers and academicians wishing to contribute toward this subject of interest.
\end{abstract}

\section{Introduction}

Catalysis and sustainability are two terms that can be related to each other in quite a significant way. As we know, catalysis acts as the one supporting the chemical industry in achieving different kinds of sustainability. Its contribution further moves on to behave as an energy transmitter which acts as a very crucial factor for fulfilling the current energy requirement. In addition to chemically catalytic processes, current progress towards renewable energy generation is based on catalytic processes employing state-of-the-art technology. Catalysis was frequently seen not only as a component and a pillar in chemicals, but as a tool in the chemical business. In a world where rising numbers of people have all the necessary resources, sustaining the demand and supply ratio, sustainable society demands balance. 
Aside from its scientific importance, nanoscience is surrounded by a lot of hype. Fantastic claims have been made concerning quicker computers, lower-cost manufacturing, and medical discoveries. Tennis rackets, selfcleaning autos, paints, and cosmetics are among the products predicted to include nanotechnology.

A number of instances show instead that novel innovation results from the development of catalysis specifically in the chemical sector which worked toward fulfilling the sustainable development goals. For instance, studies have showcased a microwave-assisted fast technique to produce graphene nanosheets and zinc nanocomposites. It should be emphasized that the microwave approach not only accelerates nucleation and development but also allows for greater control when engineering anisotropic nanostructures over a carbon core [1]. A similar approach was given for the synthesis of $\mathrm{TiO}_{2}$ nanotripods which were further used for degrading dyes [2].

Hydrocarbon processing is important as we have many hydrocarbon-based compounds which we use in our daily lives. From aviation fuel to polymers to even false teeth, hydrocarbon-based compounds or a processed form of it are present. Hence, having well-established processes and production lines is important, and many industries which work with hydrocarbons are dependent on them. Appropriate catalysts ensure this and provide us with efficient conversion rates. In the hydrocarbon section of this review article, we will be looking at the various catalysts and nanocatalysts currently in use and also being researched upon, their performances in experiments, the conditions in which they work well, and the output they produce.

This review provides an insight into the use of nanomaterials as a greener, more efficient catalyst replacement for the toxic, corrosive traditional stoichiometric reagents. Onepot synthesis approach which is designed to make the production process simpler, faster, and minimize production costs is also explored. Green engineering has been in conjunction with nanomaterials since nanomaterials can be synthesized using renewable feedstocks, and in turn, nanomaterials can be used to replace catalysts that employ strong reaction conditions and produce a lot of waste. Nanomaterials are also used in environmental remediation. One such novel methodology was used by Rashid et al., where they explored fluoride removal using graphene oxideceria nanohybrids $\left(\mathrm{GO}-\mathrm{CeO}_{2}\right)$. Fluorides are known to be the leading cause of dental issues in people, so treating them before drinking is critical. The nanohybrid produced displayed ultrarapid kinetics for fluoride removal, with equilibrium achieved in 1 minute, one of the quickest kinetics for fluoride removal recorded to date [3]. The use of nanomaterials in biomedical applications as nanocarriers in drug encapsulation is also discussed. A comparison between the different catalytic reactions used has been drawn.

\section{Understanding Catalysis}

When a catalyst is used to accelerate the rate of the chemical reaction, it is called catalysis. Catalysis can be either heterogenous or homogenous in nature. Most chemical and processing industries use catalysis. Catalysts, as proved by the second law of thermodynamics, do not change the extent of the chemical reaction or influence the reaction. They rather only quicken the rate of the reaction by reducing the activation energy for the reaction to carry on. Some of the widely used catalysts are aluminosilicates, vanadium, iron, platina + alumina, and nickel. They have multiple uses from catalytic cracking in the crude hydrocarbon industry to producing ammonia in Haber's process. Mechanism: catalysts change the activation energy of a reaction and hence quicken the rate at which the reaction happens. Activation energy is the minimum energy required for the reaction to proceed. They lower the activation energy required by orienting the particles in order to get more successful collisions or by reacting with the substance to form a highly reactive intermediate and then come back to its natural form after the reaction is done and the products have been formed. Pickering emulsions stabilized by solid emulsifiers and which have high interfacial area are one of the recent developments in the field of catalysis.

There are usually two types of catalysts present, namely, homogeneous and heterogeneous catalysts. A heterogeneous catalyst works in a separate phase than its reactants, whereas a homogeneous catalyst works in the same phase. Solid heterogeneous catalysts function within gaseous or liquid reactants in the vast majority of cases. It has active areas on its surface, which the reactants adsorb to before interacting with the catalyst. The reactivity of the molecules is improved as a result of this contact. The difference between both is provided in (Table 1).

The goal of catalysis science research in the past was to optimise turnover rates. Selectivity to generate the intended product without forming byproducts will be a big research problem in the future. We need to improve our understanding of the molecular components of selectivity. For exact control of size, structure, position of additives, and location of catalyst particles on supports, new synthetic methods of catalyst manufacture are required. The development of certain novel characterization techniques, modelling, and fabrication methods have turned catalysis into a trendy topic of discussion. With the future generation of scientists, scholars, students, and researchers, this trend will continue to rise.

There has been significant progress in the solid emulsifier design, the scope of reactions that can be catalyzed, and in the engineering aspects of Pickering emulsions. Homogeneous organomagnesium, lanthanides, and group 2 metals show great reactivity as catalysts. Their availability, low cost, nontoxicity, and ecologic advantages show great potential for them to be turned into viable catalysts [9]. Redox catalysis has been the next big thing. They are an alternative when electrode passivation and over-reaction pose a problem during reaction control. Redox catalysts have good potential in applications such as battery research, optoelectronics, and photoelectron transfer processes [10]. Artificial switchable catalysts which are equipped with smart structural features respond reversibly to light, $\mathrm{pH}$, redox events, etc. Hence, these catalysts provide us with a good degree of control over the outcome of catalytic reactions 
TABLE 1: Comparison of homogeneous and heterogeneous catalysts.

\begin{tabular}{|c|c|c|c|}
\hline Properties & Homogeneous catalyst & Heterogeneous catalyst & Reference \\
\hline $\begin{array}{l}\text { Operating } \\
\text { temperature }\end{array}$ & $\begin{array}{l}\text { In most cases, at low temperatures, the inclusion of a } \\
\text { solvent, which is required to dissolve the catalyst, may } \\
\text { reduce the reaction temperature, but it also mandates } \\
\text { the use of mild conditions. }\end{array}$ & Wide range of temperatures, which are mostly high & {$[4]$} \\
\hline Active sites & $\begin{array}{l}\text { They are, for the most part, well-defined. The vast } \\
\text { majority of catalysts are made up of single-atom } \\
\text { transition metals that are held together by ligands. }\end{array}$ & $\begin{array}{c}\text { The vast majority of the time, it is ill-defined. When } \\
\text { dealing with metal particles on a support, the active } \\
\text { site may represent only a small percentage of the } \\
\text { metal. }\end{array}$ & {$[5]$} \\
\hline Selectivity & $\begin{array}{l}\text { High due to the simplicity with which the catalyst's } \\
\text { steric and electronic characteristics can be fine-tuned }\end{array}$ & $\begin{array}{l}\text { Low because controlling the steric and electrical } \\
\text { characteristics of the catalyst is more complex }\end{array}$ & {$[6]$} \\
\hline Recyclability & $\begin{array}{l}\text { Given the fact that the complexity of the catalyst } \\
\text { treatment, it is costly }\end{array}$ & $\begin{array}{l}\text { Even though the catalyst may need to be treated, } \\
\text { recycling is quite straightforward }\end{array}$ & [7] \\
\hline $\begin{array}{l}\text { Removal of } \\
\text { catalyst }\end{array}$ & $\begin{array}{c}\text { Separating the catalyst from the product is costly, as it } \\
\text { is with recyclability }\end{array}$ & $\begin{array}{l}\text { As previously said, catalyst separation from the } \\
\text { product, like recycling, is simple }\end{array}$ & {$[8]$} \\
\hline $\begin{array}{l}\text { Removal of } \\
\text { product }\end{array}$ & $\begin{array}{l}\text { An organic solvent with a low boiling temperature is } \\
\text { used for product extraction. As a catalytic mixture, } \\
\text { the aqueous solution can be reused. }\end{array}$ & $\begin{array}{l}\text { They have the advantage of being easily eliminated by } \\
\text { simple filtration at the end of the reaction. The } \\
\text { outcome, in theory, is contaminant-free, allowing the } \\
\text { catalyst to be recycled into the following step. }\end{array}$ & [6] \\
\hline
\end{tabular}

[11]. However, in the rest of the paper, we will be talking indepth about the latest advancements in the field of nanomaterials regarding catalysis.

\subsection{Catalysis in Fine Chemical Synthesis}

2.1.1. Understanding Fine Chemicals. Fine chemicals are pure substances with high added value manufactured in comparatively small amounts. The classification of fine chemicals can be made into active pharmaceutical ingredients (API), agrochemicals, and high-purity specialty chemicals. Fine chemicals have a much higher E factor than bulk chemicals, which means much more unwanted products produced need to be discarded or recycled in fine chemical production, even though the annual production is lesser than bulk chemicals. Traditionally, fine chemicals are produced by organic synthesis using stoichiometric reagents. Catalysts are to be used instead of stoichiometric reagents according to green chemistry.

It is important to keep in mind the characteristics of fine chemicals in order to synthesize fine chemicals. They are unstable at elevated temperatures and susceptible to changes in their environment. Fine chemical synthesis methods need to be flexible due to the diversity of types and the relatively small volumes required. The production that takes place in batch multipurpose plants involves multistep syntheses with short product lives. The degree of utilization of the reactants must be optimal to make the process profitable, achieved by selectivity. Selectivity is important in separating the product from the side products. Catalysis is significant in increasing selectivity.

2.1.2. Catalysis in Fine Chemical Synthesis. Acid catalysis is a highly used reaction in the fine chemical industry. The catalysts which are utilized must be recovered and reused so that we can get maximum utilization. Catalysis provides improved production processes and removes or transforms unwanted or toxic byproducts. Good selectivity and activity at low temperature is required to produce fine chemicals.

Catalysts can be either homogeneous or heterogeneous. Industries usually prefer homogenous catalysts for asymmetric synthesis because they have high turnover frequencies (TOFs) along with good enantioselectivities. However, homogeneous catalysis has certain drawbacks such as volatile pollutant emissions, need for waste management, and inability to recover catalysts. Supports are used to immobilize homogenous catalysts to help in product/catalyst separation and promote the reaction through higher acidity. Commonly used supports for catalysts in fine chemical synthesis are charcoal, silica, and alumina.

In an attempt to synthesise and efficient catalyst for hydrogenation of arenes, Hong Yang et al. used metal catalyst support to contain the tethered complex $\mathrm{Rh}(\mathrm{N}-\mathrm{P}) /$ $\mathrm{Pd}-\mathrm{SiO}_{2}$ and $\mathrm{Rh}(\mathrm{N}-\mathrm{N}) / \mathrm{Pd}-\mathrm{SiO}_{2}$, which showed better activities than the usual homogenous catalysts used for this purpose [12]. However, immobilized catalysts are costly and have limited access. They have lower selectivity, and their activity reduces with reuse, and (metal) active species are subjected to leaching in solution [13].

The catalysts employed in the reactions in the fine chemical synthesis must be extremely selective and efficient in order to minimize wastage and make the process profitable. It is easier to separate heterogeneous catalysts from the reaction products. Sometimes, they do not even need to be separated at all. They are easy to recover and reuse. Moving from homogeneous to heterogeneous catalysis solves waste disposal problems while simplifying steps used in production such as recycling and neutralization. Heterogeneous catalysis is usually used to produce bulk chemicals, and noncatalytic organic synthesis is used in fine chemical synthesis.

Mineral acids like sulfuric acid and organic liquid acids like p-toluenesulfonic acids have had a prolonged existence in the fine chemical synthesis industries as catalysts. However, there is a scope for making the process greener by 
its replacement with solid catalysts. The advantage of shifting from liquid to solid catalysts for fine chemical production includes the possibility of reuse, reduction in waste, and there is no need to neutralise the products. Synthesis of some of the catalysts currently being employed in fine chemicals is shown in Table 2, and their applications are shown in Table 3 .

A lot of hydrogenation reactions have been found to rely on metal-based catalysts. Organometallic complexes are used in hydrogenation reactions in fine chemical synthesis. They are also used in cross-coupling reactions such as Suzuki-Miyaura coupling, Sonogashira coupling, and Buchwald-Hartwig amination. Ruthenium, platinum, and palladium can homogeneously catalyze carbonylation of olefins, alcohols, amines, and phenols in fine chemical synthesis at mild conditions more efficiently.

\subsection{Catalysis in Hydrocarbon Processing}

2.2.1. Catalytic Cracking. The naphtha fraction and the gas oil fraction from the fractional distillation of crude contain the largest hydrocarbon molecules. Catalytic cracking is the breaking up of large hydrocarbons into smaller units. These bits are then easier for further processing. Olefins and hydrocarbons, which have lesser molecules and light in nature, are preferred in the petrochemical industry. For cracking to occur in comparatively lower temperatures, catalysts have to be used. Catalytic cracking or thermal catalytic cracking (TCC) requires lesser temperatures compared to thermal cracking and hence has lesser energy requirements.

\subsubsection{Oxide-Based Catalysts. Oxide catalysts such as} modified alumina catalysts impregnated with potassium carbonate $(0-8.8 \%)$, when operated in a fixed bed reactor (at the conditions of $\left.760-82^{\circ} \mathrm{C}, 1 \mathrm{~atm}\right)$, show reduced coke deposition. This is a good thing as coke formation leads to problems such as poisoning and sintering. However, it was also noticed that production rate of methane, ethylene, and propylene had decreased as well. The next catalyst is $\mathrm{KVO}_{3}$ impregnated alpha- $\mathrm{Al}_{2} \mathrm{O}_{3}$ which was again operated in a fixed bed reactor. When naphtha was cracked using this, yields of propylene and ethylene increased to about $10 \%$ from the initial 5\%. It was noted that addition of $\mathrm{B}_{2} \mathrm{O}_{3}$ increases the interaction between $\mathrm{KVO}_{3}$ and alpha- $\mathrm{Al}_{2} \mathrm{O}_{3}$. The coke generation on the catalyst surface during pyrolysis, increased with reaction temperature and axial length and decreased with catalyst amount. $n$-Hexane over $\mathrm{MoO}_{2}$ showed an increase in the selectivity for olefins but a decrease for alkanes when tested in the temperature range of $557-640^{\circ} \mathrm{C}$. $5 \mathrm{~mm}$ alpha- $\mathrm{Al}_{2} \mathrm{O}_{3}$ spheres were explored for their catalytic activity in the catalytic cracking of naphtha. Although there was an increase in the yield of ethylene and propylene, the deposition of coke on the catalytic surface increased along with the axial length. The coke formation was influenced by the physical properties of the alpha- $\mathrm{Al}_{2} \mathrm{O}_{3}$ spheres. $n$-Heptane over different calcium aluminates in a fixed bed reactor showed significant reduction of coke deposition on the catalyst's surface. The process which was done here was steam pyrolysis. Modified alumina $/ \mathrm{K}_{2} \mathrm{CO}_{3}$, $\mathrm{KVO}_{3} / \mathrm{Al}_{2} \mathrm{O}_{3}, \quad n$-hexane $/ \mathrm{MoO}_{2}$, alpha- $\mathrm{Al}_{2} \mathrm{O}_{3}$ spheres, $n$-heptane $/ \mathrm{CaAl}_{2} \mathrm{O}_{4}$ were reacted in a fixed bed reactor and gave the following products: reduced coke deposition: propylene (10\%) and ethylene (10\%); selectivity for olefins increased: propylene (15\%) and ethylene (15\%); and reduced coke deposition [30].

2.2.3. Zeolites. Zeolites are one of the most commonly used catalysts in hydrocarbon processing. Zeolites can be obtained naturally, or they can also be produced synthetically. Synthetic zeolites tend to have more desirable structures and chemical features. The significance of zeolites and their unique characteristics which make them beneficial are discussed as follows [31]:

(1) They exhibit high thermal, hydrothermal stability, and frequent oxidative regeneration.

(2) They are crystalline microporous solids; hence, they are capable of allowing a high concentration of reactant molecules inside their cages.

(3) Zeolites can be produced in various structures with pore openings consisting from 8 to 12 member rings. These pore openings are often similar to the shape and size of hydrocarbons and hence form the fundamental of catalytic shape-selective behaviour of zeolites. The mesoporous system of zeolites was also found to have a say in the transport of reactants or products.

(4) Zeolites and related materials can usually be produced using a variety of chemicals.

(5) Zeolites have ion-exchange properties which enables them to be used in detergents. This is because of their higher acidity content which can later be adjusted to fit the framework of aluminium [32].

HZSM-5 is a common type of zeolite-based catalyst commonly used in catalytic cracking of hydrocarbons. The important thing with HZSM-5 is to have the correct Si/Al ratio. This ratio usually varies depending on the material to be cracked. Cyclohexane was cracked with HZSM-5 with varying $\mathrm{Si} / \mathrm{Al}$ ratios and temperatures. This experiment showed better yield of the expected products. HZSM-5 having different structures such as hierarchical (h-HZSM5) and crystalline (c-HZSM-5) where tested as catalysts in the catalytic deoxygenation of oleic acid, at $300^{\circ} \mathrm{C}$. All showed more than $99 \%$ conversion of oleic acid. h-HZSM-5 showed 3 times greater hydrocarbon yield compared to the other variants. This was due to the enhanced accessibility to acidic sites in its hierarchical structure. It was observed that when the pore size is decreased in HZSM-5, selectivity of lighter olefins increases [9]. $n$-Hexane isomerization using various Pt-loaded catalysts were compared in which $\mathrm{Pt} / \mathrm{H}$-beta showed a conversion of $72 \%, \mathrm{Pt} / \mathrm{HM}$ showed a conversion of $14.2 \%$, and Pt/HZSM-5 showed $46 \%$. $\mathrm{Pt} / \mathrm{H}$-beta also showed the highest selectivity to isomerization at $95.6 \%$ followed by Pt/HZSM-5 at $89.4 \%$ and then $\mathrm{Pt} / \mathrm{HM}$ at $81.3 \%$ [33]. 
TABLE 2: Synthesis methods of some of the catalysts used in fine chemical synthesis.

\begin{tabular}{|c|c|c|c|}
\hline $\begin{array}{l}\text { Catalyst used in the } \\
\text { FCS }\end{array}$ & Synthesis of the catalyst used & Reaction conditions & Reference \\
\hline Zeolites & $\begin{array}{l}\text { It is prepared from sodium aluminate, silica colloidal } \\
\text { solution, } \mathrm{NaOH}, \mathrm{H}_{2} \mathrm{SO}_{4} \text {, and tetrapropylammonium } \\
\text { bromide }\end{array}$ & $\begin{array}{l}\text { Hydrothermal method takes place at } 90-180^{\circ} \mathrm{C} \text {, } \\
\text { at a pressure of } 15 \text { bars using water as a solvent }\end{array}$ & {$[14]$} \\
\hline Titanium silicates & $\begin{array}{l}\text { Tetrabutyltitanate is slowly added to sodium } \\
\text { hydroxide dissolved in distilled water and stirred } \\
\text { vigorously. Aqueous hydrogen peroxide is added after } \\
\text { stirring, and a yellow solution is obtained to which } \\
\text { TPABr is added. After this, fumed silica is } \\
\text { incorporated into the solution. The mixture is } \\
\text { crystallized, and the titanium silicate crystals are } \\
\text { filtered, washed, and dried. }\end{array}$ & $\begin{array}{l}\text { The reaction takes place at room temperature, } \\
\text { and then, it is crystallised at a temperature of } \\
\qquad 180^{\circ} \mathrm{C}\end{array}$ & {$[15]$} \\
\hline $\begin{array}{l}\text { Nugent-RajanBabu } \\
\text { reagent }\end{array}$ & $\begin{array}{l}\text { Titanocene dichloride is reduced with metal-reducing } \\
\text { agents to give the green Ti(III) chloride bridged } \\
\text { dimer. This undergoes further reactions with } \\
\text { acetonitrile to make the blue acetonitrile coordinated } \\
\text { titanocene complex. }\end{array}$ & The reaction takes place at room temperature & {$[16]$} \\
\hline $\begin{array}{l}\text { Amine- } \\
\text { functionalized } \\
\text { mesoporous silica }\end{array}$ & $\begin{array}{l}\text { Pluronic }(4 \mathrm{~g}) \text { and } \mathrm{NaCl}(11 \mathrm{~g}) \text { are dissolved in } \mathrm{HCl} \\
\text { solution and stirred. TEOS is added, and the solution } \\
\text { is hydrolyzed for one or two hours before slowly } \\
\text { adding the organic silane. This mixture is then filtered, } \\
\text { and the solid product is dried at RTP overnight. Then, } \\
\text { it is refluxed in ethanol for a day. The product is } \\
\text { filtered, washed with water and ethanol, and dried. }\end{array}$ & $\begin{array}{l}\text { The mixture is then stirred at } 40^{\circ} \mathrm{C} \text { for } 20 \mathrm{~h} \text {, } \\
\text { then poured into a polypropylene bottle, and } \\
\text { aged at } 90^{\circ} \mathrm{C} \text { under static condition for } 24 \mathrm{~h}\end{array}$ & [17] \\
\hline
\end{tabular}

Table 3: Application of some catalysts in fine chemical synthesis.

\begin{tabular}{lll}
\hline $\begin{array}{l}\text { Sr. } \\
\text { no }\end{array}$ & Catalyst used in the FCS & Application in FCS \\
\hline
\end{tabular}

\begin{tabular}{lll}
\hline 1 & Titanium silicates & Synthesis of paracetamol and caprolactam \\
& Industrial oxidation and ammoxidation type processes
\end{tabular}

2 Hydrotalcite

3 Titanium

4 Nugent-RajanBabu reagent

5 Amine-functionalized mesoporous silica

Phosphotungstic acid

$6 \quad\left(\mathrm{H}_{3} \mathrm{PW}_{12} \mathrm{O}_{40}\right)$

$7 \quad \mathrm{TiO}_{2}$

$8 \quad$ Montmorillonite K10 (solid bronsted acid)

9 Acid resins like amberlyst-15 or

9 Nafion SAC-13 15 Synthesis of $\gamma$-lactones carbonates potassium hydroxide Inert gas gloveboxes

Flavanone synthesis

Production of camphor
(1) The products obtained from the hydroxylation reaction of phenol reaction are used in flavoring agents, pesticides, photographic chemicals, dyes and pigments, antioxidants, rubber processing, leather tanning, and polymerization inhibitors and in drugs and pharmaceuticals

(2) Efficient catalysts for the condensation reactions like aldol condensations, Knoevenagel condensation, and Claisen-Schmidt condensation reactions

(3) Used as a catalysts precursor in many reactions

(4) In production of benzaldehyde of natural essence by retroaldol condensation reaction of cinnamaldehyde

(5) Ketone oxidation to ester

(6) Transesterification of glycerol with diethyl carbonate to obtain glycerol

(7) They are capable of replacing conventional base catalysts like sodium and

(8) Epoxidation of unsaturated ketones

(9) Transesterification of rape oil with methanol

Hydroaminoalkylation, formal redox catalysis and hydroamination reactions

(1) Used in omeprazole synthesis

(2) Formation of methyl- $\alpha, \beta$-glucosides from cellulose

(1) One-pot reaction between phthalaldehydic acid and substituted ketones using microwave-assisted methodology to obtain phthalides

(2) One-pot reaction between 2,5-dimethoxytetrahydrofuran and aromatic amide to produce $\mathrm{N}$-Acylindoles 
TABLE 3: Continued.

\begin{tabular}{|c|c|c|c|}
\hline $\begin{array}{l}\text { Sr. } \\
\text { no }\end{array}$ & Catalyst used in the FCS & Application in FCS & Reference \\
\hline 10 & $\mathrm{ZnCl}_{2}$ supported on $\mathrm{SiO}_{2}$ & Production of octahydroacridines & {$[24]$} \\
\hline 11 & acid zeolites & $\begin{array}{l}\text { Production of ortho-hydroxyacetophenones which is used to make salicylic acid } \\
\text { and para-hydroxyacetophenones, an intermediate in production of paracetamol }\end{array}$ & [25] \\
\hline 12 & HY zeolite & Formation of nitriles from aldehydes & [26] \\
\hline 13 & $\mathrm{AlCl}_{3}$ & 2-Methylanthraquinone synthesis & [27] \\
\hline 14 & $\mathrm{InBr}_{3}$ & Synthesis of $\alpha$-silyloxy- $\gamma$-indolyl nitriles & [28] \\
\hline 15 & Sn- and Zr-beta zeolites & $\begin{array}{l}\text { In synthesizing } 4 \text {-methoxybenzyl 1-methylpropyl ether having a pear scent. Using } \\
\text { these catalysts for this reaction is advantageous because the problem of water } \\
\text { formation which might affect the efficiency of the process is removed since they } \\
\text { have hydrophobic surfaces. }\end{array}$ & [29] \\
\hline
\end{tabular}

Binary cobalt/zeolite catalysts when used during the production of synthetic high-octane gasoline (by FischerTropsch method) revealed that the presence of a zeolite component limits the overheating of the catalyst in the catalyst zone and hence reducing collateral reactions and carbon decomposition. Binary catalytic systems having zeolite bases showed high stable activity as well. In the chemical and petrochemical industries, hydrogenation of naphthalene is a common method for producing solvents that may be used to store hydrogen in proton-exchange membranes (PEMs) and fuel cells. When a variety of catalysts were tested to see which one performed the best, it was discovered that transition metal catalysts outperformed noble metal catalysts like platinum because transition metals are less expensive. Zeolite was discovered to be an excellent catalyst for this purpose, but the main disadvantage was that it possessed micropores, which might cause poor diffusion.

Two primary zeolite-based catalysts, cobalt over ZSM-5 and $\mathrm{Ni} / \mathrm{HY}$, were chosen and compared based on their distinct characteristics. The findings were compared to those obtained with traditional Co-Ni catalysts. When compared to $\mathrm{Co} / \mathrm{ZSM}-5$, the $\mathrm{Ni} / \mathrm{HY}$ catalyst had a greater naphthalene conversion, a longer life, and improved tetralin selectivity. The activity of $\mathrm{Ni} / \mathrm{HY}$ remained constant over time, whereas the commercial NiMo/alumina catalyst showed modest deactivation. Although the conversion of the $\mathrm{Co} / \mathrm{Silica}$ catalyst was high during the first hour of the process, it declined considerably beyond that time due to coke deposition, which was aided by the high catalyst acidity [34].

Furthermore, several types of studies were carried out to determine their morphological distinctions. In comparison with other catalysts, TGA examination of used catalyst samples indicated a higher coke deposit on the $\mathrm{Ni} / \mathrm{HY}$ catalytic reaction. This demonstrates the overall quantity of coke was not connected to the catalyst's total acidity, but rather to the catalyst's activity in converting naphthalene. The catalytic process of forming HCs from carbon monoxide and hydrogen, also known as syngas, is a complex catalytic process involving a significant number of sequential and parallel reactions. In the presence of a catalyst based on group VIII transition metals, the process is operated at normal or increased pressure (mainly iron or cobalt). The Fischer-Tropsch synthesis can be thought of as carbon monoxide reductive oligomerization. Alkanes, alkenes, and oxygenated compounds are the end products.
Hydrogenation of $\mathrm{CO}$ to $\mathrm{CH}_{4}$; disproportionation of $\mathrm{CO}$ to generate carbon dioxide; and water gas shift process, which primarily involves the reduction of syngas to carbon dioxide and hydrogen, are some of the additional reactions that could be involved. Secondary reactions such as the generation of lower alkenes and alcohols, hydrogenation of alkenes, dehydrogenation of alcohols, partial hydrocracking of primary alkanes, and internal hydrogen transfer can occur over the whole temperature range in which paraffins from syngas are synthesised. The equilibrium composition of hydrocarbons synthesised from $\mathrm{CO}$ and $\mathrm{H}_{2}$ differs greatly from the actual product composition. The Fischer-Tropsch reaction is a kinetically regulated reaction.

It was found that cobalt catalyst complexes for fabricating alkanes from syngas are pretty ideal. Studying the active sites of $\mathrm{Co}$ and $\mathrm{Ni}$ catalysts for hydrocarbon synthesis from syngas can provide insight into the relationships between catalytic activity and selectivity on the one hand and structure of active areas on the surface of cobalt catalysts on the other hand. The synthesis of hydrocarbons from syngas over metal-based catalysts occurs in a multiphase system with syngas, hydrocarbons, and carbon dioxide in the gas phase. The liquid phase is made up of liquid C5-C18 hydrocarbons and dissolved solid C19+ products, and water, while the solid phase is made up of a catalyst. Ergo, the result mainly involves increasing the contact of these compounds with the catalyst and at the same time, promoting any other reactions which might come through [35].

2 main sites, according to Lee et al., was found to be the most active with respect to the conversion of syngas to hydrocarbons. The first one is the sites wherein the sorption of $\mathrm{CO}_{2}$ takes place via the dissociative routes, to produce carbon, which in turn, when hydrogenated, produces methane. The second one has two different parts to it: the metal itself and its oxide. The metal is as is, and the oxide acts as a support. $\mathrm{CO}_{2}$ is adsorbed at the metal site post which it moves toward the support, i.e., the oxide part to form metal formyl complexes. Similar is the gas with hydrogen, wherein after getting adsorbed by the metal, comes to the support to react with the formyl complex to form $\mathrm{CH}_{x} \mathrm{O}$. Aromatic hydrocarbons are formed on Co catalysts via synthesis from $\mathrm{CO}$ and $\mathrm{H}_{2}$. This is an unusual occurrence. When a synthesis is carried out using bifunctional catalysts at a high temperature, these chemicals are generated. On Co-zeolite catalyst systems, aromatic hydrocarbons can occur. The 
hydrocarbon synthesis from $\mathrm{CO}$ and $\mathrm{H}_{2}$ is mediated by the metal sites of such catalysts. On zeolite acid sites, the resultant olefins are aromatized. The rate at which aromatic hydrocarbons are formed is governed by the rate at which olefins are formed [36].

2.2.4. SAPO-34. SAPO catalysts are basically silicon added to molecular sieves of aluminophosphate (ALPO). ALPO is usually weak, and hence, silicon is added. Although being very similar to zeolite, SAPO catalysts have Al-O-P structure instead of $\mathrm{Si}-\mathrm{O}-\mathrm{Si} / \mathrm{Si}-\mathrm{O}-\mathrm{Al}$ structures found in zeolites. SAPO-34 variant was found to be having highest selectivity over others. SAPO-34 has a shape of chabazite with a pore size opening of 3.5-6 A. In a study where the SAPO-34, HZSM-5, and another catalyst called $\mathrm{H}$-modernite were tested in the catalytic cracking of C4-olefins, HZSM-5 showed the highest yield of light olefins. H-modernite had the least stability, but it was more acidic than the other two. During the study of catalytic cracking of butane using SAPO-34 and other zeolites, SAPO-34 showed the highest selectivity to light olefins but also exhibited deactivation much quicker than the other catalysts. This quick deactivation was attributed to its smaller pore sizes. Lastly, SAPO34 and HZSM-5 injected with cerium and lanthanum were studied for their catalytic activity. Results showed that, about $0.8 \%$ wt. of lanthanum and $8 \%$ wt. of cerium at $750^{\circ} \mathrm{C}$ produced the maximum yield of propylene, and changing cerium's percentage alone to $12 \%$ wt. produced the maxim yield of ethylene [30].

2.2.5. Catalysts Used in Alkene Oligomerization. Alkene oligomerization is used to convert alkenes which are gaseous in nature in ambient conditions and later convert into products which are liquid in nature. The mechanism of alkene oligomerization has been demonstrated in Figure 1. Oligomerization is important as it helps in producing heavier olefinic compounds such as motor fuels, pharmaceuticals, and detergents from light alkenes. For this, many compounds such as solid phosphoric acid (SPA), acid resins such as amberlyst line of products, amorphous silica-alumina, zeolitic materials such as crystalline and microporous zeolites, and lastly some nonacidic solids have been tested as potential catalysts. Metal catalysts such as aluminium, nickel, group IV metals, group V metals, and cobalt- and iron-based catalysts have also been tested. The abovementioned catalysts show good catalytic activity. The mechanism of oligomerization has been depicted as follows (Figure 1) [37].

Since the early 1930s, solid phosphoric acid catalysis has been employed in industry to produce "polymer gasoline" by oligomerizing light olefins. SPA catalysts are traditionally made by combining phosphoric acid with kieselguhr (diatomaceous earth), extrusion, and high-temperature calcination. The resulting catalyst is a "glassy layer" of catalytically active phosphoric acid species $\left(\mathrm{H}_{3} \mathrm{PO}_{4}, \mathrm{H}_{4} \mathrm{P}_{2} \mathrm{O}_{7}\right.$, and polyphosphoric acid) atop a substrate of silica and other silicon phosphates. An improved form of SPA catalyst was synthesized by Coetzee et al. The effect of acid concentration, impregnation temperature, calcination temperature, and water addition was tested. This improved catalyst showed higher conversion rates and life time than commercially available SPA catalysts. For instance, commercial SPA catalysts clocked about $475 \mathrm{~kg}$ of product $/ \mathrm{kg}$ of catalyst, and the synthesized and improved ones produced about $620 \mathrm{~kg}$ of product $/ \mathrm{kg}$ of catalyst [38].

Antunes et al. review the various types of resin catalysts used in oligomerization. Nafion resins were shown to have higher conversion levels compared to SPA in the propene oligomerization. Macroporous sulfonic resins were analysed and showed good conversion rates when mixed with isobutene. The pressure required to keep all the reactants in, and liquid phase was also lesser in this case. Various other processes such as butene oligomerization, isobutene dimerization, isobutene trimerization, isoamylene dimerization, and isoamylene trimerization were studied by the paper. Many types of resin-based catalysts were used in them. One notable type was amberlyst (macroporous ion exchange resin) catalysts, which showed high rates of conversion and selectivity [39].

$\mathrm{N}$-heterocyclic carbenes (NHCs) are established and effective ancillary ligands on homogeneous metal catalysts. Different types of NHCs such as simple monodentate NHCs, metal complex NHCs, Grubbs-type NHC-Ru, and others were tested with early transition (Sc, Y, Ti, Zr, V, Lu, and $\mathrm{Ho}$ ), midtransition ( $\mathrm{Co}, \mathrm{Fe}$, and $\mathrm{Ir}$ ), and late transition (Ni and $\mathrm{Pd}$ ) metals by forming complexes with them and then using them as catalysts in polymerisation and oligomerization reactions. This review was conducted by McGuiness, and varying results were noted for each combination [40].

\subsection{Catalysis in Renewable Energy}

2.3.1. Significance of Catalysis in Renewable Energy. As energy transporters and carbon resources, it is pretty obvious that the feedstock of coal, petroleum, natural gas, and alternative fuels is driving the energy systems right now. The entire market value chain depends on the latter. With the amount of feedstock being generated, the process of taking raw materials, adding value to them through production, fabrication, and other means to create a finished final product and selling it has become easier. Their use comprises either direct burning which can produce heat or other means of burning to produce chemical value chain goods such as polymers, textiles, medicines, cosmetics, and dyes. This is pretty ideal given that these two processes combined or not combined, eventually convert the product into $\mathrm{CO}_{2}$, which has been using its power to store energy from the Sun for the longest time. It is quite self-explanatory that the pace at which the $\mathrm{CO}_{2}$ liberation is taking place, precisely around 40 Gigatons per year, is placing this generation on an unsustainable course [41].

In the twenty-first century, we are facing a lot of challenges, the majority of which are related to resource availability, change of climate, demand and supply of energy, and process efficiency for more sustainable growth. Future generations will be required to solve these issues through a 

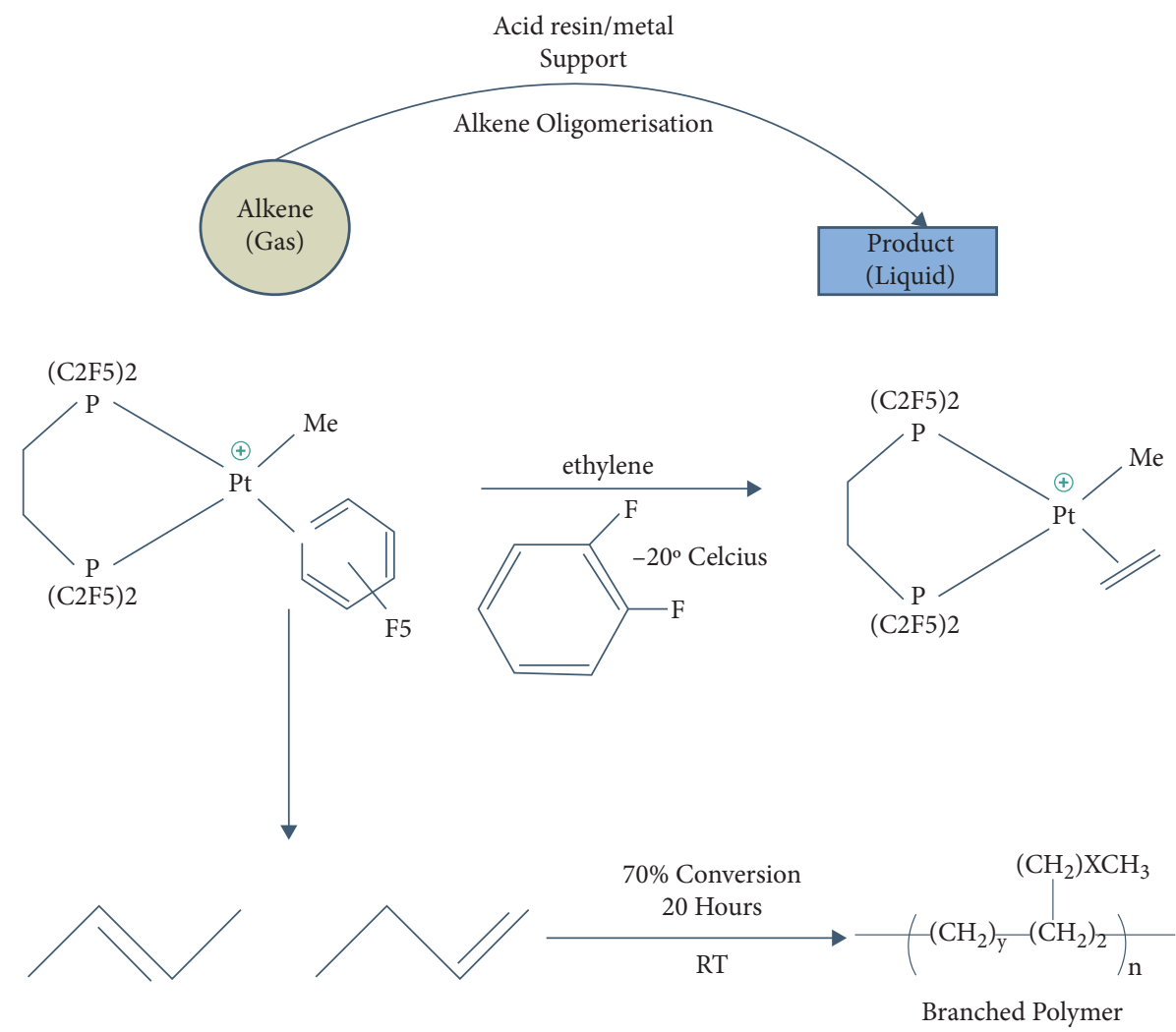

FIGURE 1: Mechanism of alkene oligomerisation.

multidisciplinary approach involving disciplines such as biotechnology, biochemistry and material sciences, as well as unparalleled coordinated monitoring and modelling of a wide range of variables, from social to economic to environmental. As we know, most of the existing methods for making potential electrocatalysts need the use of dangerous and costly precursors, external models, and chemicals containing heteroatoms, which ultimately raise costs and restrict their economic applications. Converting natural resources into carbon-based products or modifying inexpensive industrial graphite for a better product to address these disadvantages are opening new horizons as successful methods for engineering materials that are more sustainable, thereby being environmentally friendly, with state-of-the-art electrocatalytic properties [42]. Various $\mathrm{CO}_{2}$ routes for renewable-based products have been demonstrated in (Figure 2).

The conventional industries which are particularly based on oil-derived chemicals are in depletion right now, which enables the fabrication of new materials which are made from biomass such as levulinic acid and glycerol [43]. It was reported that the levulinic acid when made to pass through hydrogenation along with $\mathrm{CuAg} / \mathrm{Al}_{2} \mathrm{O}_{3}$ as a bimetallic catalyst achieved as good as $100 \%$ yield to g-valerolactone even when the same process was carried out for about 9 times. Moreover, levulinic acid was proposed as one of the main constituents for synthetizing polyrridones. Reductive amination of levulinic acid in the presence of graphitic carbon nitride, with the catalyst being platinum was found to have a selectivity greater than $60 \%$ [44].

\subsection{2. $\mathrm{CO}_{2}$ Reduction to Fuels}

(1) $\mathrm{CO}_{2}$ to Methanol. Syngas to methanol is a process that is known quite well commercially. This takes place using $\mathrm{Cu}-$ $\mathrm{Zn}$-oxide catalysts. Starting with $\mathrm{CO}_{2}$, the same catalysts can be used. The classic method employs a system involving 2 catalysts, which are mainly copper and their oxides to catalyze RWGS (reverse water gas shift), followed by a CO reduction to methanol catalyst. A common multicomponent catalytic system $\mathrm{Cu} / \mathrm{ZnO} / \mathrm{ZrO}_{2}$ was found to exhibit good productivity and stability. Nonetheless, the complex equilibria involving $\mathrm{CO}, \mathrm{CO}_{2}, \mathrm{H}_{2} \mathrm{O}$, and $\mathrm{CH}_{3} \mathrm{OH}$ make comparing catalysts problematic because the performances are highly reliant on feed composition. Sometimes though, water can act as an inhibitor to this process. As an alternative, the option is to develop new types of catalysts that are particular for methanol synthesis from $\mathrm{CO}_{2}$, which on water inhibition do not become vulnerable, and may involve a different than the conventional mechanism that does not pass through the first step of the RWGS reaction, which is syngas production.

Low-temperature methanol synthesis, also known as LTMS, has emerged to be a superb alternate technique to solid phase catalysts carrying a gas-phase process. LTMS is based on the hydrogenation of $\mathrm{CH}_{3} \mathrm{OH}$ to $\mathrm{CH}_{2} \mathrm{O}_{2}$, which can be further used for different applications. LTMS is settled using catalysts followed by etherification to form methyl formate which is the methyl ester of formic acid as an alternative to methyl formate (initially synthesized from syngas). Similarly, LTMS also involves the catalytic hydrogenation of methyl 


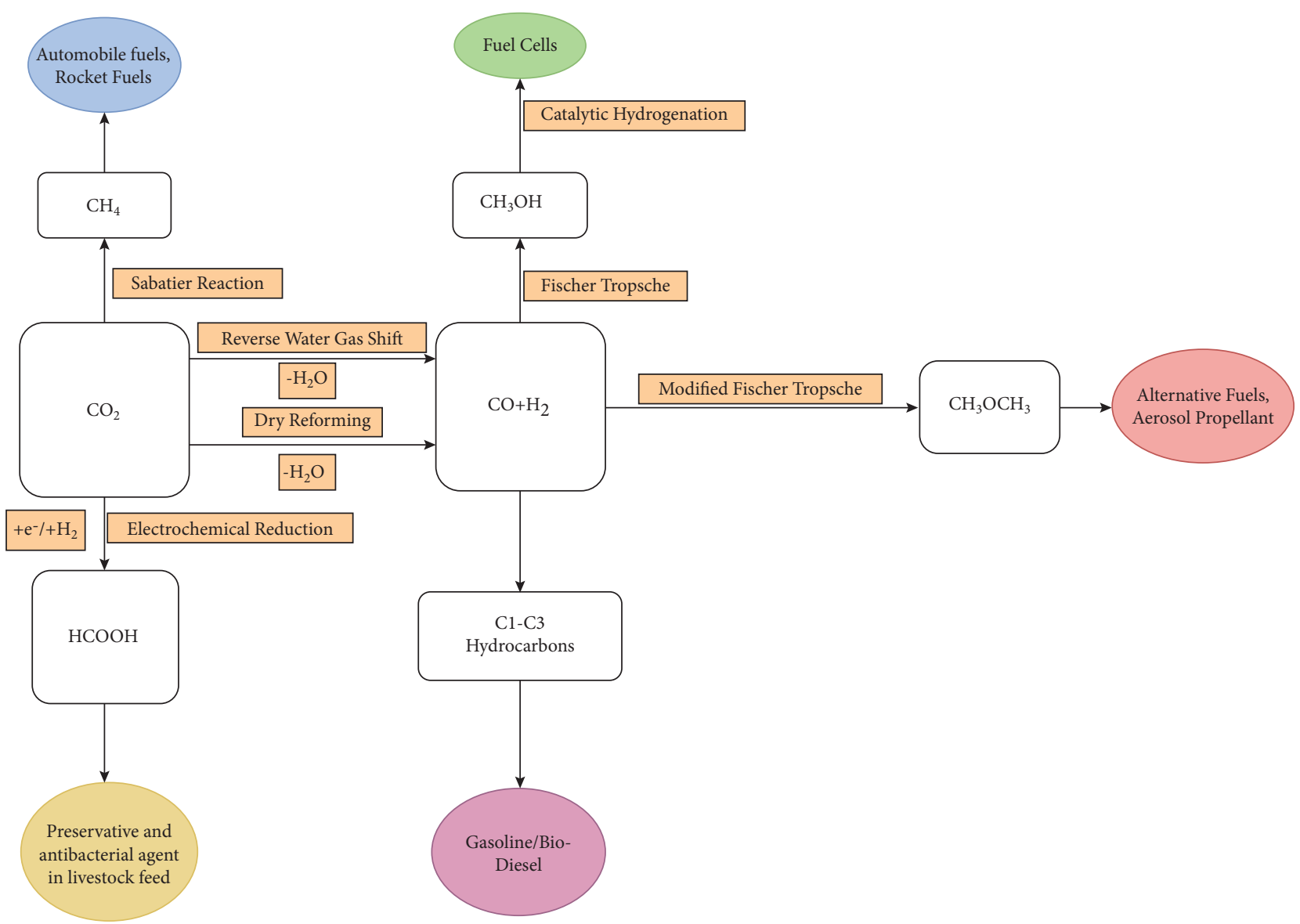

FIgURE 2: Flowchart describing $\mathrm{CO}_{2}$ routes for renewable-based products.

formate to form 2 different $\mathrm{CH}_{3} \mathrm{OH}$ molecules. Ruthenium complexes which are based on Pincer-type ligands can also be used to carry this process out. These catalysts can efficiently hydrogenate organic compounds such as carbamates, formats, and carbonates even with decent circumstances, opening up novel avenues for the production of methanol through the hydrogenation of these compounds, which can be generated from $\mathrm{CO}_{2}$ and $\mathrm{CO}$.

(2) $\mathrm{CO}_{2}$ to Short-Chain Olefins. The reverse water gas shift (RWGS) is usually used to convert $\mathrm{CO}_{2}$ to short chain olefins, and it is frequently enhanced by the same catalysts used in the subsequent processes. However, due to thermodynamic restrictions, developing direct $\mathrm{CO}_{2}$ conversion pathways would be preferred. It was demonstrated that utilising FischerTropsch Fe/K catalysts coated atop cerium-modified alumina, around $62 \%$ selectivity of C2-C5 was achieved and a greater number of hydrocarbons in one step at approximately 50 percent $\mathrm{CO}_{2}$ conversion rate. Sai Prasad et al. converted a CO/ $\mathrm{CO}_{2} / \mathrm{H}_{2}$ syngas using a copper/aluminium catalyst at $1 \mathrm{~atm}$. The $\mathrm{C}_{2}-\mathrm{C}_{4}$ fraction had over $85 \%$ selectivity to alkene (about $40 \%$ of the total hydrocarbon fraction), which was substantially higher than usual. Methanol-to-propylene (MTP) process was recently carried out for the first time in China before slowly being developed to be known as one of the best commercial processes for synthesizing methanol. It is mainly established on the utilisation of a zeolite catalyst capable of providing a yield measuring to approximately 69\% (with about 96 percent selectivity) at approximately $425^{\circ} \mathrm{C}$ and near 1 atm. By altering the equilibrium with hybrid catalysts, methanol systems can be extended to DME (dimethyl ether, having a high cetane number thereby used as a replacement for different liquid fuels) [45].

(3) $\mathrm{CO}_{2}$ to FT (Fischer-Tropsch) Products. Several recent research studies have focused on the influence of $\mathrm{CO}_{2}$ on Fischer-Tropsch synthesis, which transforms water gas into liquid hydrocarbons. When the feed was changed from syngas to $\mathrm{CO}_{2}+\mathrm{H}_{2}$, there was a considerable increase in methane production for cobalt and iron catalysts. For Fe/Co catalysts, similar results were obtained. It was also discovered that the product distributions of $\mathrm{CO}$ and $\mathrm{CO}_{2}$ hydrogenation varied [46].

(4) $\mathrm{CO}_{2}$ to Methane. The catalysts employed are comparable to those used in $\mathrm{CO}_{2}$ reforming. At low temperatures, 10 wt. $\% \mathrm{Ni} / \mathrm{CeO}_{2}$ has a strong activity of $\mathrm{CO}_{2}$ conversion and a high $\mathrm{CH}_{4}$ selectivity. Another effective catalyst for reforming, $\mathrm{Rh} /-\mathrm{Al}_{2} \mathrm{O}_{3}$ catalysts also function well in $\mathrm{CO}_{2}$ methanation at low temperatures. As a result, there is a relationship between $\mathrm{CO}_{2}$ and $\mathrm{CO}$ methanation catalysts, the latter of which is frequently based on supported $\mathrm{Ru}$ catalysts, as $\mathrm{Ru}$-doped ceria also has remarkable $\mathrm{CO}_{2}$ methanation performance. Although no particular method of operation 
has been found out to be efficacious for maintaining the stability of NPs, particle size still is considered as an important factor when creating highly active catalysts [47].

(5) $\mathrm{CO}_{2}$ to Formic Acid. Formic acid, or $\mathrm{HCOOH}$, is very useful for the environment in terms of its use in cattle feed as a preservative and antibacterial agent. It is frequently used to maintain winter feed for cattle when sprayed on fresh hay, clovers, oats, or any other silage. It helps to stop the degradation of the feedstock as well as lets it to retain its nutritious content for a longer than usual time. There are currently few reports available on converting $\mathrm{CO}_{2}$ to formic acid. Homogeneous catalysts, on the other hand, have proven the most successful in converting $\mathrm{CO}_{2}$ to derivatives of formic acids, such as formamides. Ionic liquids are used to aid in the electrocatalytic conversion of $\mathrm{CO}_{2}$ to FA. While the initial hydrogenation of $\mathrm{CO}_{2}$ into formamides quite a while back, the usage of supercritical $\mathrm{CO}_{2}$ and chelating phosphine has substantially enhanced the reaction speeds [48]. Generally, catalysts can be used to convert $\mathrm{CO}_{2}$ into various products as shown in Table 4.

Typically, noble metal catalysts are utilized in devices which help promote the clean energy conversion with respect to ORR (oxygen reduction reaction) and OER (oxygen evolution reaction) to boost these crucial but inactive chemical reactions. Moreover, the goal is to reach a good amount of efficiency of these devices, which can only be done by using noble metal catalysts. COFs (covalent organic frameworks) as an alternative to noble metal-based catalysts are a recent expansion in the hunt for efficient and profitable ORR and OER catalysts. Given that 2D COFs have a similar structure as graphene (conjugated macromolecular design), they can be used to create lithium-ion batteries or solar cells by regulating functional groups [52].

Carbon dioxide reduction is currently popular due to the fact that $\mathrm{CO}_{2}$ emissions cause global warming by functioning as a greenhouse gas. Metal-related materials are the most commonly used electrocatalysts for $\mathrm{CO}_{2}$ conversion. Some of them, however, are unstable and easily degraded in water, limiting their application in aquatic media. COF derivatives were recently discovered to be excellent electrocatalysts for converting $\mathrm{CO}_{2}$ to $\mathrm{CO}$.

The imine condensation of cobalt (Co (TAP)) terephthaldehyde yielded the Co-COF. After that, the activated product, i.e., $\mathrm{COF}$ was put on a porous conducting carbon fabric. Under applied potentials, the electrolytes were made to react in a $\mathrm{CO}_{2}$-saturated aqueous bicarbonate buffer. The only identifiable carbon-based product was carbon monoxide. When the end product's qualities were examined using a SEM, it was discovered that there was no discernible change in the crystallinity or morphology of the final product. More unique structures of COF derivatives are predicted to be synthesized, potentially increasing the efficiency of carbon dioxide reduction [52].

2.3.3. Water Splitting. For turning solar energy into hydrogen, water splitting via photocatalytic route is found to be a potentially expansible and a profitable technique. Given the fact that semiconductors with either of the two reactions, that is, $\mathrm{H}_{2} \mathrm{O}$ reduction or oxidation, can be used for water splitting, Z-scheme systems which are based on a two-step process, involving photoexcitation of a hydrogen evolution photocatalyst (HEP) and an oxygen evolution photocatalyst (OEP) are well adapted. However, a systematic transmission of electrons between HEP and OEP particles is difficult to achieve.

The photocatalyst taken into consideration was divided based on HEP and OEP as follows:

(i) $\mathrm{SrTiO}_{3}: \mathrm{La}$ as a HEP

(ii) $\mathrm{BiVO}_{4}$ : $\mathrm{Mo}$ as an OEP

(iii) Embedding is done in $\mathrm{Au}$ and $\mathrm{Rh}$

The conduction band has photoexcited electrons, and the valence band consists of positive holes. Through gold, the transfer of electrons takes place from the conduction band of the OEP to the donor band of HEP with the embedding taking place at $\mathrm{Rh}$. Meanwhile, excited electrons in $\mathrm{BiVO}_{4}$ reduce $\mathrm{H}_{2} \mathrm{O}$ to $\mathrm{H}_{2}$, thereby acting as a HER cocatalyst, while holes in $\mathrm{BiVO}_{4}$ oxidize $\mathrm{H}_{2} \mathrm{O}$ to $\mathrm{O}_{2}$, acting as an OER cocatalyst, resulting in total water splitting. Therefore, enabling the transfer of charge through the Au layer should essentially improve the photocatalyst sheet's overall water splitting performance [53].

Water splitting by photocatalysis, photovoltaic-driven electrolysis, and photo electrolysis are all examples of chemical-friendly pathways for HER. The most direct route of HER is photocatalysis, in which sunshine is utilized directly to convert $\mathrm{H}_{2} \mathrm{O}$ to $\mathrm{H}_{2}$. It, however, has low energy absorption and conversion efficiency in terms of solar energy along with a slow reaction rate. Heat has recently been shown to overcome the foregoing constraints, resulting in improved photocatalytic performance. Photocatalysis is the most straightforward path, in which sunlight directly converts $\mathrm{H}_{2} \mathrm{O}$ into $\mathrm{H}_{2}$. However, it has low efficiency when it comes to the absorption and conversion of energy, as well as a slow reaction rate. Heat has recently been shown to overcome the foregoing constraints, resulting in an improved performance in photocatalysis.

Furthermore, photovoltaic-driven electrochemical water splitting has gained a lot of interest because of its controllability, long-term prolificacy, and reaction upgradeability with less expensive and more efficient PV cells. However, because of the significant overpotentials, future efficiency improvement is restricted and must be addressed. Heat may reduce the required energy for electrocatalytic $\mathrm{H}_{2}$ evolution, which is being investigated to overcome the above concerns [54].

Initially, $\mathrm{MoS}_{2}$ was nonreactive to HER; therefore, was first dismissed as a viable hydrogen evolution electrocatalyst. However, it was discovered in 2005 that the edge structure of $\mathrm{MoS}_{2}$ was very similar to the one found in the nitrogenase. In addition, from the energy diagram, it was found that these $\mathrm{MoS}_{2}$ edge-based structures demonstrated computational free energy of atomic hydrogen bonding which was comparable to that of platinum, implying that $\mathrm{MoS}_{2}$ could be a potential HER electrocatalyst in theory. To improve the 
TABLE 4: Novel catalysts that are used to convert $\mathrm{CO}_{2}$ into the respective products and their applications.

\begin{tabular}{lcccc}
\hline Sr. no. & Catalyst & Product & Application of product & Reference \\
\hline 1 & $\mathrm{Cu} / \mathrm{ZnO} / \mathrm{Al}_{2} \mathrm{O}_{3}$ & $\mathrm{CH}_{3} \mathrm{OH}$ & Feedstock to produce chemicals & [46] \\
2 & Pincer-type ruthenium $(\mathrm{II})$ & $\mathrm{CH}_{3} \mathrm{OH}$ & Feedstock to produce chemicals & Manufacture of polymers \\
3 & $\mathrm{Fe} / \mathrm{Mn} / \mathrm{K} /$ alumina & $\mathrm{C}_{2}-\mathrm{C}_{5} \mathrm{HCs}$ & Manufacture of polymers \\
4 & $\mathrm{Fe} / \mathrm{Cu} / \mathrm{Al} / \mathrm{K}$ & $\mathrm{C}_{2}-\mathrm{C}_{5} \mathrm{HCs}$ & [48] & [41] \\
5 & $\mathrm{ZSM}-5$ zeolite & Propylene & Polypropylene for plastic products. \\
6 & $\mathrm{Fe} / \mathrm{Co}(\mathrm{FT}$ synthesis & Liquid $\mathrm{HCs}$ & Feedstock in petrochemical plants & {$[49]$} \\
7 & $\mathrm{Ni} / \mathrm{CeO}_{2}$ & $\mathrm{CH}_{4}$ & Fuel for heat and light & Fuel for heat and light \\
8 & $\mathrm{Rh} /-\mathrm{Al}_{2} \mathrm{O}_{3}$ & $\mathrm{CH}_{4}$ & Fuel for heat and light & {$[50]$} \\
9 & $\mathrm{Ru}-$ doped ceria & $\mathrm{CH}_{4}$ & $\mathrm{CH}_{2} \mathrm{O}_{2}$ & Preservative in livestock feed \\
\hline
\end{tabular}

catalytic performance of $\mathrm{MoS}_{2}$, a traditional but effective method, was to create a porous structure in them to increase their specific surface areas. $\mathrm{MoS}_{2}$ with a nanoporous structure can be actively pursued to maximize the number of the exposed active sites [55]. Apart from having a greater surface area, the porous structure may actually give certain benefits also, including a wider area of contact and adequate reactant and product transport. Similarly, doping an external element into the lattice structure allows for the engineering of the material's structures to improve the HER performance. Like sulphides, metal selenides, carbides, nitrides, and phosphides were also showing a similar performance in terms of water splitting [56].

The selection of a low-cost metal core is critical for designing a molecular catalyst which performs well, for HER. Fe, $\mathrm{Mn}, \mathrm{Cu}$, and Mo-based molecular HER catalysts are in short supply. Cobalt and nickel complexes, on the other hand, have been extensively researched, presumably due to their high activity as a catalyst in the HER. Nickel is more common on the planet and biorelevant, making research into nickel-based molecular catalysts more appealing. Second, the design of the ligand scaffold as well as modification procedures is important, as this can help to stabilize a low-oxidation-state metal and reduce the needed overpotential [57].

2.3.4. Environmental Treatment. Organic pollutants such as volatile organic compounds, 4-nitrophenol, are extensively present in the air and water, posing a major threat to the ecosystem. We should develop a method for degradation of such pollutants via the catalytic route that is both sustainable and efficient, and photothermocatalysis appears to be a viable option. As the name suggests, this mechanism uses both light and heat from solar energy for pollution indemnification. There are quite a few ways of reducing VOCs. VOCs are significant air pollutants that are toxic to both human health and the environment. PTC has been in interest of the researchers given the fact that it can exploit sunlight's UV spectra for efficient VOC reduction at low temperatures and with a faster rate of reaction [54].

A tremendous effort has been made to find out ways in which VOCs can be mitigated to enhance the environmental treatment. Initially, the catalytic activity of $\mathrm{TiO}_{2}$ (underlayer on Hg lamp) for photothermocatalytic oxidation of benzene without the use of an extra heater was investigated, with the lamp serving as both a light/heat source. $k_{\mathrm{PTC}}$ of $\mathrm{TiO}_{2}$ is fairly high when compared to the total of $k_{\mathrm{PC}}$ and $k_{\mathrm{TC}}$ of the latter, implying that it belongs to PTCC due to the combined use of UV light and the heating effect generated from the underlaying $\mathrm{Hg}$ (photo thermal cocatalysis) [58].

It is found that, under the radiation arriving from natural sunlight, the $\mathrm{Nd}^{3+} / \mathrm{NiFe}_{2} \mathrm{O}_{4}$ system was a propitious material in terms of its photocatalytic ability. The inert $\mathrm{NiFe}_{2} \mathrm{O}_{4}$ is converted into a highly solar light active photocatalyst when neodymium is substituted into the $\mathrm{NiFe}_{2} \mathrm{O}_{4}$. This is because the nickel ferrite band gap has shrunk. Furthermore, $\mathrm{NiFe}_{2 x} \mathrm{Nd}_{x} \mathrm{O}_{4}$ photocatalysts are inexpensive, recyclable, and recoverable photocatalysts with high effectiveness (up to approximately 97\%) for decomposition of various organic contaminants. The concentration of $\mathrm{Nd}^{3+}$ substitution was increased to achieve substantial visible light absorption in order to construct an efficient photocatalyst [59].

To improve the catalytic ability ultimately to promote environment protection, we can imbibe the concept of supports. Given the fact that the metal fraction in touch with the support increases, the effect of such interactions on catalysts becomes more pronounced as the metal cluster or particle size decreases. These interactions change the state of the catalysts electronically, thereby affecting the catalytic activity as well as durability at an ambient temperature [60].

Carbon monoxide is usually produced when fossil fuels are burned incompletely. $\mathrm{Cu}_{2} \mathrm{O}$ and $\mathrm{CuO}$, on the other hand, usually have high activity when it comes to oxidizing CO. After investigating the performance of copper oxides catalyst, it was found that the catalytic activities of these two were in the order $\mathrm{Cu}_{2} \mathrm{O}>\mathrm{CuO}$, with the capacity to seize or release the surface lattice associated. The higher surface concentration oxygen and resulting enhanced interplay with $\mathrm{CO}$ provided to the considerably increased activity on the samples employed when compared to fresh $\mathrm{Cu}_{2} \mathrm{O}$ catalysts. In the oxidation of $\mathrm{CO}$, the shape also plays a crucial effect [61].

The reduction of environmentally hazardous $\mathrm{NO}_{x}$ released by power plants is difficult, especially in the realm of catalysis. Given that the nitrogen oxides $\left(\mathrm{NO}_{x}\right)$ create a variety of environmental issues, including acid rain and smog (mixture of VOCs and other pollutants) in city-like regions, the removal of $\mathrm{NO}_{x}$ has gotten a lot of attention in recent decades. Extensive study has been done on selective catalytic reduction (SCR) of $\mathrm{NO}_{x}$ which utilizes $\mathrm{NH}_{3}$. The standard catalyst for commercial $\mathrm{NO}_{x}$ SCR using ammonia $\left(\mathrm{NH}_{3} \mathrm{SCR}\right)$ is $\mathrm{V}_{2} \mathrm{O}_{5} \mathrm{WO}_{3} / \mathrm{TiO}_{2}$. 
VOCs are key contributors to secondary PMs and atmospheric oxidants and are generated by a variety of industrial operations and transportation activities. Oxidation via catalytic routes is one of the most advanced ways for removing VOCs since it uses less energy and produces less $\mathrm{NO}_{x}$ as compared to thermal oxidation. $\mathrm{Co}$ and $\mathrm{Cu}$ oxides are inexpensive and high-performance proxies to catalysts made of noble metals for oxidation of VOC. In the oxidation of hydrocarbons, copper oxides usually have high catalytic activity. $\mathrm{Cu}_{2} \mathrm{O}$ 's morphology and form have been proven to influence the $\mathrm{CH}_{x}$ oxidation activity [62].

Organic pollutants in waste water from industrial activities can harm not only just the aquatic life but also the ecosystem as a whole. The majority of the chemicals found in wastewater is carcinogenic and must be removed. Advanced oxidation processes (AOPs), which are based on the formation of reactive oxygen species (ROS), are found pretty productive for degrading such harmful organic contaminants in wastewater among other water remediation technologies. Heterogeneous catalysts have garnered more attention than homogeneous catalysts mainly because of their ease of separation as well as recycling from the liquid reaction environment. The use of heterogeneous Fenton-like reactions to degrade persistent organic pollutants has obtained a lot of interest. In aqueous solution, the Fenton reaction utilizes $\mathrm{Fe}^{2+} / \mathrm{Fe}^{3+}$ and $\mathrm{H}_{2} \mathrm{O}_{2}$ to form one of the most powerful oxidants, $\mathrm{OH}$ radical. $\mathrm{Cu}$ uses the Fenton methods to follow a similar pattern. In the presence of $\mathrm{H}_{2} \mathrm{O}_{2}$ and organic contaminants, a copper catalyst can perform a Fenton-like reaction [61].

Due to the substantial harm done to the human body and the environment, soot particulates generated by diesel engines have obtained a lot of attention. $\mathrm{CuO}$ has been demonstrated to be very efficient for soot combustion via catalytic routes. Sometimes, oxides including mixed oxides and perovskite-like oxides also do a good job to catalyze the combustion of soot. The catalytic activity for soot combustion very much depends on the shape of the catalyst. Ergo, the activity can be increased by designing the catalyst shape carefully [61].

2.3.5. Fuel Cell. Fuel cells, which use electrochemical reactions to convert a fuel's chemical energy into electricity, are one of the essential enabling technologies for the necessary conversion to a hydrogen-based economy. Polymer electrolyte fuel cells (PEFCs), one of the most important fuel cells under research today, have been identified as a prospective power source in the future for zero-emission automobiles [61].

Binary alloy catalysts such as Pt-Co and Pt-Ni were developed to better understand where the increased activity in the ORR comes from. Various methodologies were used to characterize these catalysts. The particle size of the catalysts was changed and modified by thermal treatment to study the particle size effect properly. The particle size rose as the temperature of the thermal treatment increased, whereas the surface area dropped. It was found that when the surface area decreases, the specific activity of the said Pt-based alloy catalysts increased. Furthermore, due to the reduced Pt-Pt adjacent distance, the Pt-Co and Pt-Ni binary alloy catalysts had higher activity when compared to a single Pt having the same surface area as the latter [63].

As far as PEFCs are concerned, keeping aside the expensive cost of the Pt catalysts, PEFCs have poor performance durability, which is primarily caused by electrode active surface area loss as well as carbon support corrosion. The Pt cathode in a direct methanol fuel cell (DMFC) suffers a performance loss due to its inability to tolerate the diffusion of methanol from the anode side through the membrane of the cell. As a result, whether we utilize $\mathrm{H}_{2}$ fuel or $\mathrm{CH}_{3} \mathrm{OH}$ fuel, PEFCs require efficient, long-lasting, and, most significantly, low-cost catalysts to replace $\mathrm{Pt}$ and Pt-based materials [63].

One method is to make catalysts out of cheap metals like palladium or ruthenium. Despite the fact that Pt is avoided, the effect is that one valuable metal is replaced with another that is, on the whole, less active than Pt. Replace Pt with a catalyst which is abundant and nonprecious which are not subjected to price inflation in high-demand situations as an alternative. As a result, this class of composites can be used to make a number of additional nonprecious composite materials that can be used as catalysts for the PEFC cathode [63].

\section{Nanomaterials in Catalysis}

Nanocatalysis is an emerging field connecting homogenous and heterogeneous catalysis. Nanomaterials are finding large number of applications in catalytic reactions owing to a great number of surface atoms and several other unique characteristics. As a result, the surface area of nanomaterial plays governing aspect in determining their activity and selectivity. Nanomaterials possess dominant optical, electrical, optical, and mechanical properties. As a result, they find extensive functions in various fields, including electronic, magnetic and optoelectronic, biomedical, pharmaceutical, cosmetic, energy, catalytic, and materials. Enhancements made in the relative surface area of the nanomaterial and quantum effects modify the physicochemical characteristics of nanomaterials. The catalytic performance of the nanomaterials is improved by using, naked monometallic, bimetallic, and multimetallic nanomaterials reinforced with porous and stable supports.

3.1. Significance of Nanomaterial Properties in Catalysis. Over the years, nanomaterials have found a huge number of applications in the fields of science and technology. On the basis of different structural modifications of the nanocatalyst, radovic has classified the nanostructure (CNFs/CNTs) into (i) graphitic carbon atoms with flat $\mathrm{sp}^{3}$ hybridization; (ii) allotropes of carbon like nanofibers made of carbon atoms with curved $\mathrm{sp}^{2}$ hybridization; and (iii) carbon allotrope with $\mathrm{sp}^{3}$ hybridization, for example, diamond [64]. Compared to other allotropic forms, nanofibers of carbon containing curved $\mathrm{sp}^{2}$ hybridization; despite containing curved $\mathrm{sp}^{2}$-hybridized carbon atoms, have shown 
notably different nanostructures and properties. Compared to nanofibers, in nanotubes, the hexagonal substructures are present in a centered cylindrical plane in an axial alignment, owing to the differential alignment of carbon atoms in the underlying planes. Even though both CNTs and CNFs possess analogous characteristic properties of a typical carbon materials, for example, activated charcoal and CBNP (carbon black nanoparticle), they offer enhanced catalytic properties due to characteristic features, such as fibrous/ tubular structure, stronger resistivity to acidity and basicity, stronger electrical conductance, higher exterior surface area ranging from $80 \mathrm{~m}^{2} / \mathrm{g}$ to $200 \mathrm{~m}^{2} / \mathrm{g}$, ensuring higher catalytic activity, definite structure, alterable properties of the mesoporous structure, and high structural stability [65].

Since the number of edge sites in CNFs is greater, it acts as a catalyst precursor anchoring site. Through structural engineering and altering the properties of the interface, the nanoframework of the carbon atoms can be adjusted with respect to its primary and secondary nanostructures. This provides new possibilities of tuning the properties of the external surface of carbon nanofiber and the supporting metal nanoparticle [65]. This is done by means of surfaceoxidation [66], foreign-ion doping (using nitrogen, boron etc.) [65], and confinement-effect [65]. Due to large facial area and increased electrothermal conductivity in its crystalline structure, CNTs and CNFs offer better catalytic activity compared to traditional catalysts such as alumina and silica [67]. This results in a better interaction between CNFs/ CNTs and metal-ions. The modifications introduced in the morphology of the catalyst material leads to enhancement in its selectivity and performance [65]. Due to the mesoporous structure, combined with large external surface area, there is an increase in the catalytic reactivity in case of liquids, gases and several other complex reactions [68].

3.1.1. Consequences of Textural Properties, Surface Functional Groups, and N-Doping on CNFs/CNTs. CNFs possess a mesoporous structure with large surface area (approx. $10-300 \mathrm{~m}^{2} / \mathrm{g}$ ), owing to the 3 -dimensional stacking of fibers $[65,69]$. These catalysts are designed to decrease the diffusional differences between liquid and gaseous phase, i.e., in gas-phase reactions, the transportation of the particles over the gas and diffusion of particles within the gas-filled pores are faster, compared to liquid phase. Due to the resistivity of carbon nanomaterials to acidic and basic agents, and its preferential hydrophobic nature, they find wide range of applications in liquid phase catalytic reactions. As a result, CNTs and CNFs, supported by its larger surface area and mesoporous structure, become an ideal alternative catalyst in liquid-phase reactions, as reduced mass-transport limitation is needed [70]. Liquid phase reactions using catalysts of carbon nanotubes or carbon nanofibers with a metal support are provided as follows (Table 5).

The amphoteric nature of carbonaceous surfaces enables the acidity or basicity of the solution to control the sum of surface charge within the catalytic material, in terms of proton transfer. This is supported by the propensity of the graphene edges/defects, ensuring surface functionalization.
The surface of the catalyst becomes protonated when the $\mathrm{pH}$ becomes less than the point of zero charge (PZC) or isoelectric point (IEP). Moreover, as $\mathrm{pH}$ rises above IEP or PZC, surfaces deprotonation takes place and gets negatively charged. The structure of CNF and the kind of functional groups brings variations in the zeta potential [65]. For example, oxygen groups present on the catalyst surface varies the zeta potential value, depending on the presence of acids [79]. Therefore, oxidation in acids, such as $\mathrm{HNO}_{3}$ and $\mathrm{HNO}_{3} / \mathrm{H}_{2} \mathrm{SO}_{4}$ mixtures, results in the formation of aldehydes, quinones, and carboxy groups, and generates negative value for the zeta potentials as the $\mathrm{pH}$ value rises above 2 . As the $\mathrm{pH}$ approaches 2 , the catalyst surface gets positively charged, owing to the presence of the basic groups. Formation of large number of basic phenol groups through steam/plasma oxidation leads to positively charged surface at $\mathrm{pH}$ value below 5 .

The interactive force between the positive/negative charge carrying surface of CNT/CNF and the metal precursor results in the functionalization and activation of the material. The dispersion rate and anchoring of precursors are controlled by PZC and IEP values [80]. The adsorption rate depends on the type of metal precursor, owing to the carbon-surface charge. Anion impregnation is achieved at lower $\mathrm{pH}$ value, whereas cation impregnation is achieved at higher $\mathrm{pH}$ value. Therefore, optimum $\mathrm{pH}$ value is maintained for obtaining maximum impregnation, as extremely low/high $\mathrm{pH}$ inhibits adsorption due to high ionic strength. Moreover, the potential difference between CNTs/CNFs and the metal precursor affects the colloidal deposition in the solution [81]. Due to increment in metal precursor $(\mathrm{Pt} / \mathrm{Pd})$ deposition rate, the potential difference between CNF/CNT and metal precursor increases, that is, there is an increase in the deposition of metal colloid (negatively charged) on the CNFs/CNTs with the rise in point of zero charge (PZC) or its basicity. As a result, the size of the metal nanoparticles decreases [82].

Nitrogen doping on CNTs increases its metal dispersion capability when compared to an undoped CNTs. The polar nature of the material is increased by the presence of nitrogen functional group, thus making it more hydrophilic [83]. The pyridinic nitrogen atoms donate the $\mathrm{e}^{-}$lone pairs of the nitrogen sites in a $\mathrm{sp}^{2}$ orbital hybridization in the carbon ring plane. As a result, the basicity of the carbon support increases. The metal-support interaction decreases the size of the clusters, owing to the presence of $N$ [65]. Therefore, due to the decrease in electron density through negative inductive effect against platinum, ruthenium, palladium, and silver increases due to the presence of nitrogen-groups on the CNT surface. Additionally, the silver nanoparticles, which are anchored onto nitrogen-doped MWCNTs ( $\mathrm{CN}_{\mathrm{x}} \mathrm{MWNTs}$ ), increase its catalytic activity as pyridinic $\mathrm{N}$ in $\mathrm{CN}_{x} \mathrm{MWNT}$ makes the CNTs more reactive. This is supported by an increase in chemical affinity between the $\pi$ molecular orbital and Ag clusters, owing to the surface pyridinic sites. During the dispersion of Pt particles, they get uniformly dispersed over the materials surface as they possess particle size less than that on carbon supports without nitrogen doping. Therefore, in reactions involving 
TABLE 5: Liquid-phase reactions using catalysts of CNTs/CNFs with a metal support.

\begin{tabular}{|c|c|c|c|}
\hline Catalyst & Reactant & Remark & Reference \\
\hline $\mathrm{Pd} / \mathrm{CNF}$ & Cinnamaldehyde & $\begin{array}{l}\text { The mesoporous structure of the catalyst causes an enhancement in the active and selective } \\
\text { nature of } \mathrm{C}=\mathrm{C} \text { (double bond) hydrogenation }\end{array}$ & [71] \\
\hline $\begin{array}{l}\mathrm{Pd} / \\
\mathrm{CNT}\end{array}$ & Cinnamaldehyde & $\begin{array}{l}\text { The mesoporous structure of the catalyst causes an enhancement in the active and selective } \\
\text { nature of } \mathrm{C}=\mathrm{C} \text { (double bond) hydrogenation }\end{array}$ & [71] \\
\hline $\mathrm{Pt} / \mathrm{CNF}$ & Cinnamaldehyde & $\begin{array}{l}\text { The mesoporous nanostructure results in an enrichment of catalytic performance of the } \\
\text { material }\end{array}$ & {$[72]$} \\
\hline $\mathrm{Pt} / \mathrm{CNT}$ & Cinnamaldehyde & $\begin{array}{l}\text { The mesoporous nanostructure results in an enrichment of catalytic performance of the } \\
\text { material }\end{array}$ & {$[73]$} \\
\hline $\mathrm{Pt} / \mathrm{CNT}$ & $\begin{array}{l}\text { Nitrobenzene } \\
\text { hydrogenation }\end{array}$ & $\begin{array}{l}\text { The mesoporous nanostructure results in an enrichment of catalytic performance of the } \\
\text { material }\end{array}$ & {$[74]$} \\
\hline $\mathrm{Pt} / \mathrm{CNT}$ & Aniline oxidation & $\begin{array}{c}\text { The contact between } \mathrm{C}_{6} \mathrm{H}_{5} \mathrm{NH}_{2} \text { and the active sites is facilitated by its larger surface area of } \\
\text { contact and inadequacy of micropores }\end{array}$ & {$[75]$} \\
\hline $\begin{array}{l}\mathrm{Cu} / \\
\mathrm{CNT}\end{array}$ & Aniline oxidation & $\begin{array}{c}\text { The contact between } \mathrm{C}_{6} \mathrm{H}_{5} \mathrm{NH}_{2} \text { and the active sites is facilitated by its larger surface area of } \\
\text { contact and inadequacy of micropores }\end{array}$ & {$[75]$} \\
\hline $\begin{array}{l}\mathrm{Ru} / \\
\mathrm{CNT}\end{array}$ & Aniline oxidation & $\begin{array}{c}\text { The contact between } \mathrm{C}_{6} \mathrm{H}_{5} \mathrm{NH}_{2} \text { and the active sites is facilitated by its larger surface area of } \\
\text { contact and inadequacy of micropores }\end{array}$ & {$[75]$} \\
\hline $\mathrm{Ru} / \mathrm{CNF}$ & Bromate reduction & $\begin{array}{l}\text { The macroporous structure of the catalyst entangled with the CNF layers increases the } \\
\text { mass-transfer rate to the active sites of ruthenium }\end{array}$ & {$[76]$} \\
\hline $\mathrm{Pt} / \mathrm{CNF}$ & Methanol oxidation & $\begin{array}{l}\text { The large surface area and crystalline structure of the catalyst supports the mesoporous } \\
\text { CNF making it an excellent electrode for } \mathrm{MeOH} \text { fuel cells }\end{array}$ & [77] \\
\hline $\mathrm{Pt} / \mathrm{CNT}$ & Methanol oxidation & $\begin{array}{l}\text { The large surface area and crystalline structure of the catalyst supports the mesoporous } \\
\text { CNF making it an excellent electrode for } \mathrm{MeOH} \text { fuel cells }\end{array}$ & {$[77]$} \\
\hline $\mathrm{Pt} / \mathrm{CNF}$ & Heck reaction & $\begin{array}{l}\text { High dispersion rate of Pd particles over the mesopores of CNF increases the catalytic } \\
\text { activity and low-level leaching in Heck coupling }\end{array}$ & {$[78]$} \\
\hline
\end{tabular}

oxidation of hydrogen and reduction of oxygen atoms, hydrogenation of nitroarenes etc., the metal particles show huge increase in its catalytic activity [84]. This was supported by the improvement in the active phase nucleation, increasing strength of metal/CNTs interactions.

The presence of edge sites and functional groups on the basal plane influences the electron localization and its affinity to proton. The absence of functional groups such as oxygen and nitrogen doping along the edge positions of the structure strengthens its basicity, which improves the anchoring capability of the metal nanoparticles. However, functional groups of oxygen present on the surface are necessary for the deposition to take place. N- and B-doping, alters the Fermi level of CNFs and CNTs, thus changing its electronic nature. Additionally, $\mathrm{N}$-doping was observed to enhance the selective nature for $\mathrm{C}=\mathrm{C}$ (double bond) hydrogenation [65].

Carbon nanofibers grown with impregnated iron atoms showed greater ORR activity compared to one without it, although they were less performant [85]. N-doped vertically aligned carbon nanotubes (N-vCNTs) showcased superior ORR activity compared to undoped vCNTs. Glassy N-vCNTs was observed with greater stability in ORRs which was unaffected by $\mathrm{CO}$ and methanol poisoning [86]. $\mathrm{N}$ atoms that substituted the $\mathrm{C}$ atoms in the hexagonal ring determined the amount of current needed for achieving the required reaction at the electrode preceding the simultaneous discharge of additional ions the electrode reaction along with the transfer of $\mathrm{e}^{-}$from graphene particles to the orbitals that weakens the chemical bond between the two oxygen atoms to increase the energy of the molecule relative to its separated oxygen atoms. However, pyridinic nitrogen was observed to weaken the bond between the oxygen atoms, thus improving its onset potential [86, 87]. The metallic or semiconducting properties of the nanotubes controlled the size of activation barrier in the oxygen molecule. $\mathrm{O}_{2}$ adsorption barrier was observed to be higher for pyridinic nitrogen site compared to graphitic nitrogen sites. Heteroatom (nitrogen, boron, sulfur, and phosphorous) doping in graphitic networks increased the number of active sites, thus improving its reactivity. Nitrogen-doped materials were dual-doped to increase its stability and resistivity to $\mathrm{CO}$ poisoning $[86,88]$.

\subsubsection{Metal Nanoparticles, Electronic Properties, and Pen-} tagon Defects. The binding energy between metal nanoparticles or clusters and the CNF surfaces depends upon the energy of adsorption required by the complex present over the carbon surfaces. Density functional theory (DFT) explains how a covalent bond forms between a carbon and a metal atom [89]. Experiments were conducted to study how the CNF structure affected the metal nanoparticle. For example, the strength of interaction between metal nanoparticles or clusters and the $\mathrm{CNF}$ surfaces decreases as follows: palladium/platelets $>$ palladium/fishbone $>$ palladium/tubes. Experimentally, it was observed that the surface proton affinity in metal/CNF followed the same order $[65,90]$. Orbital analysis studies conducted on the synergetic relation between carbon materials and palladium atoms suggested the formation of a strong Pd-C bond on the basal graphite plane, which was stabilized by the electrons present in the $\pi$-bonds of the nearby aromatic rings $[65,91]$. Metal oxides-carbon nanotube interaction depended upon the inequality in the electron density between inside and outside of the carbon nanotube structure. Therefore, metal oxide particles which are present inside the CNT tubes can be 
reduced easily compared to outer parts of the structure, owing to the difference in interaction between the metal oxide and the carbon nanomaterial. Since nitrogen doping elevated the rate of dispersion of the metal particles over the support surface, there is an increment in the catalytic activity in reactions, such as cinnamaldehyde hydrogenation. Additionally, it increases the stability of the catalyst when minimal leaching of metal is found. The surface functional groups weakened the interaction between CNTs/CNFs and the metal nanoparticles, owing to increased fraction of electrons at the outer rim of the graphene structure $[65,86]$.

Electronic perturbation results in the transmission of delocalized $\pi$ electrons of the graphene support to the metal particles, which influences the properties of the catalyst particles [92]. X-ray photoelectron spectroscopy (XPS) techniques have shown that the rise of support defects strengthened metal-support interactions. Scanning tunneling spectroscopy (STS) and tunneling conductance measurement experiments done on $\mathrm{Pd}$ and $\mathrm{Au}$ metal clusters revealed that those clusters with size less than $1 \mathrm{~nm}$ showcased nonmetallic properties, which was further substantiated by the fact that these small clusters varied in reactivity towards $\mathrm{CO}$, when analyzed against larger clusters [65]. Due to the positive difference in the electron density of ruthenium particles along the outer surface and interior of the carbon nanotubes, graphene sheets suffer from structural curvature leading to the transfer of $\pi$ electron density from the interior concave surface to the exterior convex surface. This results in a variation in its electric potential. This phenomenon of metal nanoparticle confinement is greater in smaller CNTs compared to larger CNTs. Nitrogen- and boron-doping, alters the Fermi level in CNFs and CNTs, thus changing its electronic nature [65]. Since boron has one electron less than carbon, during boron doping carbon atoms are substituted with boron inside a CNT, resulting in a p-type nanoconductor [65, 93]. Compared to boron-doped CNTs, nitrogen doping in CNTs results in the formation of two types of carbon-nitrogen bonds. Substitutional nitrogen doping results in the formation of n-type conducting nature and pyridinic nitrogen doping results in either $\mathrm{p}$-type conducting nature or $\mathrm{n}$-type conducting nature, which changes according to the doping level [85].

Preferential binding between clusters of metal particles and edge structures in carbon nanofiber changes the particle strain, thus tuning the electronic characteristics of the metallic nanoparticles. This is supported by the change in bond length caused by d-band shift. Due to the difference in the length of the bond present in the atoms in the cluster and the length of gap between different layers of graphene at the CNF edges, a bind is formed between the metal clusters and the edge sites of the carbon nanofiber, causing a structural reconstruction [65]. DFT studies have shown a similar situation in induced strains because of the interactive relation between the graphene and the platinum nanoparticles. During the formation of metal nanoclusters, for example, in case of platinum nanoclusters, the catalytic performance is affected by the rate at which gases such as $\mathrm{O}_{2}, \mathrm{CO}, \mathrm{OH}$, and $\mathrm{H}$ gets adsorbed on to the platinum catalyst. This results in a charge redistribution. As a result, the selectivity for hydrogenation of unsaturated aldehydes and olefins is affected by this charge redistribution [94]. The selective hydrogenation reactions using CNF-supported metallic catalysts are given in Table 6.

While conducting XPS studies on different types of nanofibers structures (platelets, tubes, and fishbones), CNFs with platelet structure showcased strongest interactions with palladium atom. This was supported by the shift in electron density from palladium to carbon atom and high dispersion of Pd particles [104]. Additionally, compared to fishbone and tubular CNFs, platelets possessed the stronger adsorption properties. This results in a difference in the performance of the palladium/carbon catalysts during selective hydrogenation. The increase in number of $\mathrm{Pd}^{2+}$ particles in the catalysts resulted in lowering of net activity of the palladium/carbon catalyst along with its selectivity. Additionally, the palladium particles get stabilized in its ionic state due to the interaction between $\mathrm{Pd}^{2+}$ ion and the ends of graphitic structure [65].

By substituting phosphorous and boron particles on the surface of the CNFs, its edge structures can be altered, thus adjusting its crystallographic arrangement [105]. For example, by blocking the zigzag sites on the surface of CNFs by using boron resulted in a decrement in the catalytic performance during the hydrogenation of $\mathrm{C}_{2} \mathrm{H}_{4}$ and $\mathrm{CH}_{3} \mathrm{CH}_{2} \mathrm{CH}=\mathrm{CH}_{2}$ using nickel. Additionally, the decrement in the electron fraction/density on the catalyst activated carbon nanofibers increased the strength of adsorption of acetylene on the exterior reaction site of the palladium [88]. However, by increasing the fraction of functional groups with acidic characteristics on the carbon fiber, its hydrogenation activity was observed to decrease. X-ray photoelectron spectroscopy (XPS) analysis performed further justified that those functional groups containing oxygen present on the surface of carbon nanofibers did not bring any significant variations to the electronic structure of metal atoms. This further proved that, during hydrogenation, the oxygen-containing surface groups gets replaced by cinnamaldehyde on the carbon support through adsorption (Figure 3).

Density function theory (DFT) was used to study how intrinsic pentagon defects altered the electrochemical characteristics of carbon nanomaterials. The redistribution of localized electrons, caused by the intrinsic pentagon defects, resulted in increased charge density in the carbon matrix, with higher oxygen affinity [106]. The pore structures developed in the carbon matrix promoted its capacitance along with its electrocatalytic activity. Additionally, nitrogen doping was observed to enhance the super capacitor properties of carbon nanomaterials.

Pentagon defect-rich carbon nanomaterials (PD-C) were designed to study the effect on intrinsic pentagon on electrochemical reactivity. It was observed that PD-C contains 3-dimensional carbon networks with porous structure with unique areas of electron diffraction patterns. The intrinsic defect (edge and pentagon defect) was confirmed through $\mathrm{X}$-ray diffraction patterns in $\mathrm{PD}-\mathrm{C}$ which was supported by a 
TABLE 6: Selective hydrogenation reactions using CNF/CNT-supported metallic catalysts.

\begin{tabular}{|c|c|c|c|}
\hline Catalysts & Reactant & Comments & Reference \\
\hline $\mathrm{Pd} / \mathrm{CNF}$ & 1,3-Butadiene & The Pd2+ ion stabilizes the catalyst, but lowers the catalyst activity & [95] \\
\hline $\mathrm{Pd} / \mathrm{CNF}$ & Acetylene & $\begin{array}{c}\text { Due to low electron density of } \mathrm{H}_{2} \mathrm{O}_{2}-\text { or } \mathrm{O}_{3^{-}} \text {, initial TOF and selectivity for ethylene is increased } \\
\text { by initial CNF }\end{array}$ & {$[96]$} \\
\hline $\mathrm{Ru} / \mathrm{CNF}$ & Paracetamol & The structure of the catalyst affects the selective nature of the catalyst & [97] \\
\hline $\begin{array}{l}\mathrm{Pt}-\mathrm{Co} / \\
\mathrm{CNT}\end{array}$ & Cinnamaldehyde & $\begin{array}{l}\text { Due to the presence of platinum or cobalt or boron sites, the activity of catalyst along with its } \\
\text { selectivity for cinnamyl alcohol is increased }\end{array}$ & [98] \\
\hline $\mathrm{Ni} / \mathrm{CNF}$ & 1-Isobutene & The activity and stereoselectivity of the catalyst are affected by the microstructure of CNF & [98] \\
\hline $\mathrm{Ni} / \mathrm{CNF}$ & Crotonaldehyde & The activity and stereoselectivity of the catalyst are affected by the microstructure of CNF & [99] \\
\hline $\mathrm{Pt} / \mathrm{CNT}$ & Cinnamaldehyde & $\begin{array}{l}\text { The selectivity for unsaturated alcohols is increased by the presence of Pt atoms at the tip of } \\
\text { CNT structure }\end{array}$ & {$[100]$} \\
\hline $\begin{array}{l}\mathrm{Pt} / \\
\mathrm{MWNT}\end{array}$ & Cinnamaldehyde & $\begin{array}{l}\text { The formation of unsaturated alcohols determines the oxygenated functionalities in the final } \\
\text { catalyst }\end{array}$ & [101] \\
\hline $\mathrm{Pt} / \mathrm{CNF}$ & Cinnamaldehyde & $\begin{array}{l}\text { Selectivity for cinnamyl alcohol is reliant on the size of the size of the particle and the presence } \\
\text { of oxygen groups on the exterior of the material }\end{array}$ & [102] \\
\hline $\mathrm{Ru} / \mathrm{CNF}$ & Cinnamaldehyde & $\begin{array}{c}\text { Selectivity for cinnamyl alcohol is reliant on the size of the size of the particle and the presence } \\
\text { of oxygen groups on the exterior of the material }\end{array}$ & [103] \\
\hline $\begin{array}{l}\mathrm{Pt} / \\
\mathrm{MWNT}\end{array}$ & Cinnamaldehyde & $\begin{array}{c}\text { Electronic effect causes a fluctuation in the MWCNT particle diameter and thus altering the } \\
\text { selectivity of the catalyst }\end{array}$ & {$[104]$} \\
\hline
\end{tabular}

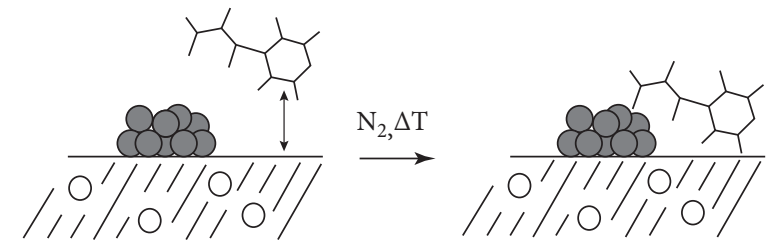

FIGURE 3: Representation of cinnamaldehyde adsorption after removing the functional groups containing oxygen, thus improving the catalytic performance of CNF supported Pt catalyst.

wider $\mathrm{D}$ band and large value of $I_{\mathrm{D}} / I_{\mathrm{G}}$, approximately 1.00 . The adsorption desorption isotherm of pentagon defect-rich carbon nanomaterials further justified that the pores present on the inner parts of the material were largely located in the mesopores and micropore regions [106]. The $\mathrm{C}$-sp $\mathrm{sp}^{3}$ bonds in the 3-dimensional carbon matrix resulted in high electrocatalytic active sites which were observed to intensify the intrinsic pentagon defects, owing to a large activity of oxygen reduction reaction. The current versus time $(I-t)$ chronoamperometric response test suggested that $\mathrm{PD}-\mathrm{C}$ structures have better immunity to $\mathrm{CH}_{3} \mathrm{OH}$ and $\mathrm{CO}$ compared to $\mathrm{D}-\mathrm{G}$ (defective graphene). It was observed that compared to common hexagons, intrinsic pentagon results in high oxygen reduction reaction. $\mathrm{PD}-\mathrm{C}$ with nitrogen doping ( $\mathrm{PD} / \mathrm{N}-\mathrm{C})$ possessed large number of active catalytic sites and oxygen adsorption energy. The rate capability curve of $\mathrm{PD} / \mathrm{N}-\mathrm{C}$ showed that the materials possessed a capacity retention of 94.5 with long-term cycling durability [106].

\subsubsection{Graphene-Based Nanomaterials and Heteroatom} Doping. Graphene (GN) based nanomaterials are used as electrode material for cathodic oxygen reduction reaction due to its high electrocatalytic conductivity, larger specific surface area (SSA), morphology, morphology of the interlayer present, and various functional groups. Nitrogen-doped GN (N-GN) showcased better catalytic activity and carbon monoxide tolerance, thus reducing the catalytic deactivation nature of CO. GN-based hybrid nanocomposite and electrocatalytic activity of GN-based nanomaterials is supported by its precursor graphene oxide (GO) [87]. Vertically aligned nitrogen-doped carbon nanotubes (VA-NCNTs) are resistant to anode-crossover and carbon monoxide poisoning and possess high catalytic activity compared $\mathrm{Pt} / \mathrm{C}$ catalyst [85]. $\mathrm{N}-\mathrm{GN}$ nanomaterials synthesis involves GO reduction followed by treatment of GO with nitrogen containing precursors. Polydiallydimethylammonium chloride (PDDA) acts as an electron acceptor in noncovalently functionalized graphene and CNTs which increases the catalytic performance of oxygen reduction reactions in fuel cells. The conductivity and electrocatalytic activity are increased by improving its SSA by one-step ultrarapid thermal expansion exfoliation process. GN-based 3D architectures (foam and aerogel) were observed to have tunable morphology and larger SSA [87]. Oxygen adsorption is facilitated by the weakening of double bond in oxygen, owing to large fraction of positive charge on the nearby carbon molecule due to the large electron density of the nitrogen atom present in the framework. Through four-electron mechanism, the pyrrolic $\mathrm{N}$ functioned as oxygen adsorbate and water reductant. The pyridinic- $\mathrm{N}$ was observed to increase its onset potential, thus converting ORR mechanism from 2 electron to 4 electron processes, while graphitic- $\mathrm{N}$ increased its limiting current density $[85,87]$. Nanotubes with vertically aligned $\mathrm{N}$ and $\mathrm{B}$ (VA-BCN) showed alterable nitrogen/boron-doping levels through thermal annealing of graphene oxide with the help of 
$\mathrm{NH}_{3}$ and $\mathrm{H}_{3} \mathrm{BO}_{3}$. Thus, obtained $\mathrm{BCN}$ and $\mathrm{GN}$ showed enhanced electrocatalytic activity due to synergic effect. Hybrid catalysts such as $\mathrm{Co}_{3} \mathrm{O}_{4} / \mathrm{N}-\mathrm{GN}, \mathrm{GN} / \mathrm{MnCo}_{2} \mathrm{O}_{4}$, $\mathrm{Co}_{(1-x)} \mathrm{S}-\mathrm{GN}$, and $\mathrm{GN} / \mathrm{Mn}_{3} \mathrm{O}_{4}$ nanocomposites showed higher ORR electrocatalytic activity and enhanced discharge capacitance.

Chemically reduced graphene oxide (CRGO) was observed with defect sites that function as traps for organic molecules, thus increasing the activity of supported catalysts. $\mathrm{N}$-heterocyclic carbene (NHC) stabilized $\mathrm{Cu}^{\prime}$-catalyst with strong NHC $\sigma$-donors and poor acceptor ligands have high catalyst activity and reactivity [107]. The dispersibility of CRGO/CNTs is improved by imidazolium groups which act as an ionic liquid moiety. The click reaction on the surface of CRGOs are adjusted by utilizing mixture of water and tertbutyl alcohol as solvent and copper(II) sulphate/sodium ascorbate as the catalyst. N-heterocyclic carbene (NHC) stabilized $\mathrm{Cu}^{\prime}$-catalyst (CRGO-lma-Cu') is obtained by proton exchanging between imidazolium moiety and $\mathrm{Cu}^{\prime}$ salt. The reaction yield and catalytic activity of CRGO-lma$\mathrm{Cu}^{\prime}$ was observed to increase (by 60\%) in the presence of tetrahydrofuran/methanol (THF/MeOH $30: 1)$ [107]. The reactivity of CNT-lma-Cu' and CGRO-lma-Cu' was compared using the equation:

$$
\text { rate }=k^{\prime} \times[\text { alkyne }]^{(1.3 \pm 0.2)} \times[\text { azide }]^{(1 \pm 0.2)} .
$$

It was observed that CNT-lma-Cu' has lower reactivity

The electronic structures of carbon nanotubes are modified via doping of p-block elements, such as nitrogen, boron, phosphorous, sulphur, silicon, selenium, fluorine, chlorine, bromine, and iodine to improve oxygen evolution reaction (OER). While studying the intrinsic catalytic activity of a catalyst material measured via overpotential (U) in ORR/OER showed better performance for catalyst with less overpotential. When graphene is doped with p-block elements, the third step in oxygen evolution reaction is the rate limiting step for doped carbon nanostructures. Relation between free energies of $3 \mathrm{rd}$ and 2 nd step in OER is $\Delta G_{3}=\left(-\Delta G_{2}\right)+C_{x}\left(C_{x}\right.$ dependent on dopant type $\left.X\right)$. However, for ORR, the rate limiting steps are first or the last step defining the correlation as $\Delta G_{4}+\Delta G_{1}=1.603-C_{x}$. [85]

Electron affinity and relative electronegativity were found as descriptor in OER/ORR reactions in terms of activity by utilizing volcano plot. The OER/ORR activity was plotted against $\phi=\left(E_{x} / E_{c}\right) \times\left(A_{x} / A_{c}\right)\left(A_{x}\right.$ and $E_{x}$ are electron affinity and electronegativity, respectively). $\phi$ is a dimension less factor representing the effects of electro negativity and electron affinity. For all dopants $X$, the overpotential exhibited volcano relationship against adsorption free energy $\triangle G O^{*}-O H^{*}$ for OER, $\triangle G O H^{*}$ for ORR and descriptors. By studying the volcano plot for $U$ (min) vs $\phi$, it was observed that nitrogen functioned as a better dopant in graphene for oxygen reduction reactions, while phosphorous showed least $U$ for oxygen evolution reaction [85]. When $\phi$ (oxygen reduction reaction) $=1-3$ and $\phi$ (oxygen evolution reaction $)=0.5-2$, the nitrogen or phosphorous doped graphene act as bifunctional catalyst with better performance compared to their noble-metal counterpart.
Elements with $\phi<1$ (example: boron, phosphorous, silicon, and antimony) acted as oxygen reduction reaction active centers. However, dopant with $\phi>1$ (example: nitrogen, sulphur, selenium, and halogens) could not do so. During nitrogen doping, more ORR and OER centers are formed in the proximity of the dopant in N-doped structures [85]. The graphene edge showed faster $\mathrm{e}^{-}$transfer rate and catalytic activity compared to basal plane of graphene since overpotential was directly proportional to the distance from the dopant to the edge.

3.1.4. Structural Properties and Catalytic Consequences. Grain boundary (GB) rich ultrathin transition metal hydroxide (TMH) network of nanosheets is synthesized via growing cobalt metal-organic framework (Co-MOFS) ZIFL-Co nanosheets on nickel foam which is then converted it into $\mathrm{Co}-\mathrm{M}(\mathrm{M}=\mathrm{Co}$ and $\mathrm{Ni}$ ) hydroxides via facile etching process. Defect engineering is utilized for increasing the number of reaction sites during electrocatalysis by creating active grain boundaries in TMO (transition metal oxides) [108]. The TMO particles are converted into ultrasmall nanocrystals, and their oxygen evolution reaction (OER) activity is increased by improving the grain boundaries using $\mathrm{Li}+$ electrochemical tuning (LiET). Thus, the GB defect and the $2 \mathrm{D}-\mathrm{GB}-$ rich ultrathin $\mathrm{TMH}$ results in high electron catalytic efficiency. The defect-rich ultrathin $\mathrm{Co}(\mathrm{OH})_{2}(\mathrm{D}-\mathrm{U}-$ $\mathrm{Co}(\mathrm{OH})_{2}$ ) possesses high electron catalytic activity for OER under alkaline conditions [108]. D-U-Co(OH) 2 was observed to have high electrocatalytic and intrinsic activity due its active surface area and double layer capacitance (Cdl) and reduced onset potential and massive cation/anion vacancy defect based on mass activity (measured at $\eta=300 \mathrm{mv}$ ).

When $\mathrm{Au}$ nanoparticles are loaded on to the surface of ceria by the method of incipient wetness impregnation process, it was observed to have smaller AU particles size and uniformly compared to the deposition-precipitation method [109]. The sample obtained via incipient wetness impregnation method showed improved performance during the catalytic oxidation of CO. This was supported by higher activity of gold nanoparticles owing to its oxidation states and smaller particle size and high dispersion. Ceria nanomaterials with different morphologies are synthesized via hydrothermal method. $\mathrm{CeO}_{2}$ showed higher catalytic activity due to high oxygen storage capacity, oxygen vacancies, and easy conversion between $\mathrm{Ce}^{3+}$ and $\mathrm{Ce}^{4+}$ ions. Nanorods and nanotubes of Ceria showed (100) and (110) surface, whereas nanotubes only showed (100) surface [109]. Carbon monoxide conversion rate was observed to be $100 \%$ in case of incipient wetness impregnation sample, $0.5 \% \mathrm{Au} /$ $\mathrm{CeO}_{2} \mathrm{~N}_{5}$ at a temperature of $70^{\circ} \mathrm{C}$ which had improved catalytic performance and stability than $5 \% \mathrm{Au} / \mathrm{CeO}_{3}$ rod and $5 \% \mathrm{Au} / \mathrm{CeO}_{2} \mathrm{NC}$ because these structures acted as anchoring sites for $\mathrm{O}_{2}$ molecules, thus showing an increased molecular oxygen adsorption and $\mathrm{CO}$ oxidation. The gold nanoparticles presence on $\mathrm{CeO}_{2}$ nanotubes was able to grow into larger size which led to its catalytic deactivation. Ceria nanotubes and nanorod showed high stability. Gold nanoparticles and bimetallic catalysts, such as Pt-Au, 
weighted on the polydopamine substrate showcased better catalytic performance to synthesize 4-aminophenol from 4-nitrophenol. Polydopamine with metal Pd (PDA-Pd), Ag, or Au loaded polymeric membranes also resulted in efficient reduction of 4-nitrophenol [110].

Metals, such as platinum, palladium, and gold, with a nanoporous structure find wide application in electrocatalytic reactions owing to its greater intrinsic catalytic activity in $\mathrm{CH}_{3} \mathrm{OH}$ oxidation, reduction of oxygen, etc. This is supported by its alterable porous size and high surface area leading to its high catalytic activity, sensitivity, and selectivity in the electrocatalytic applications. Binary nanoporous metal catalysts were observed to have high synergic effect, thus supporting its high catalytic activity [111]. Nanoporous metals ranging from microporous to macroporous are made via the method of template synthesis, which is further classified into hard template method and soft template method. Through surfactant mediated synthesis, nanoporous metals with alterable shape and size distribution are obtained [111].

The porous structure of the electrode materials used in energy systems provides it a large specific area. This facilitates the accommodation of large volume, thus improving the cycling stability and reversible energy storage capacity [111]. Currently, metals such as platinum, palladium, and gold are utilized for preparing nanoporous metals. Additionally, nonnoble porous metals, such as copper, nickel, iron, and cobalt, is also being used. During the reaction process, nanoporous metals tend to lower its surface energy, thus hindering its applications industrial processes. Therefore, it is coated using a second metal to improve its stability [111]. However, the catalytic activity of the material is improved by the complete removal surfactant during its catalytic applications.

Metal-free carbon nanomaterials are widely accepted due to its availability, environment acceptability, resistivity to corrosion, and several characteristic surface properties. They include fullerene, carbon nanotubes (CNTS), and nanodiamonds graphene sheets [112]. For carbon nanostructures, the hexagonal arrangements of carbon atoms present on the basal plane are high energy sites. The activity of these catalysts is improved by the active sites present on its surface, thus owing is high catalytic reactivity and long-term stability of oxidative dehydrogenation (ODH).

The catalytic activity of multiwalled CNTs (MWCNTs) is improved by passivation of defects with phosphorous and boron in case of ODH. However, due to longer productionretention time, the propene selectivity of $\mathrm{P}$ - and B-MWCNTs is less compared to metal oxide catalysis [112]. Aligned CNTs like N-doped carbon nanofibers prepared via high temperature treatment possess higher ORR electrocatalytic activity, due to the active sites of $\mathrm{FeN}_{2}-\mathrm{C}$ or/and $\mathrm{FeN}_{4}$-C. Metal-free nitrogen-containing CNTs (NCNTs) were observed to catalyze 4 electron ORRs more efficiently than Pt-based electrodes in alkali electrolyte [112]. The $\mathrm{N}$-induced change transfer between carbon atoms in NCNTs lowered its ORR potential. Graphene with nitrogen doping synthesized via chemical vapor deposition (CVD) by using ammonia showed similar ORR performance like metal free
NCNTs [112]. X-ray photoelectronic electroscopic (XPS) measurements are taken to decrease the presence metalcatalyst that are used for the preparing metal free NCNTs and $\mathrm{N}$-doped graphene. In polymer electrolytic fuel cell (PEMFC) with acidic electrolyte, ORR ac is increased by using NCNTs prepared via pyrolysis of acetonitrile. The catalytic reactivity metal free carbon-based catalysis is improved by doping with heteroatoms of $\mathrm{N}, \mathrm{O}, \mathrm{P}$ etc., in polymer electrolytic fuel (PEMFC) [112].

Ultrathin 2D layered metal hydroxides (LMHs) show high performance in electrocatalysis, photocatalysis, and supercapacitors. Layered metal hydroxides (LMHs), containing layered single metal hydroxides (LSHs) and layered double hydroxides (LDHs), are classified into two: $\mathrm{M}(\mathrm{OH})_{2}$, containing divalent metal cations which are coordinated by six $\mathrm{OH}^{-}$ions $\left(\mathrm{M}(\mathrm{OH})_{6}\right)$, which share the edges to create $2 \mathrm{D}$ charge-neutral layers without any intercalated species. The metal cations occupy the centers of edge-sharing octahedra. The second one, $\mathrm{M}(\mathrm{OH})_{2-\mathrm{m} x} \mathrm{~A}_{x}{ }^{\mathrm{m}-} \cdot \mathrm{nH} 2 \mathrm{O}\left([\mathrm{A}]^{\mathrm{m}-}\right.$ represents $\mathrm{Cl}^{-}, \mathrm{NO}_{3}{ }^{2-}$ ), which reconstitute $\mathrm{OH}^{-}$ions and replace it with molecules of water. Ultrathin LMHs or LMH monolayers are synthesized via exfoliation of their bulk counterparts [113]. Due to an easy control over the morphology and structure of LHMs, ultrathin layered metal hydroxides are synthesized using the bottom-up method. Modifications are made by using molecules, combinations with nanoparticles and 1D nanowires, and by assembly with other two-dimensional materials, to create hybrids. Ultrathin layered metal hydroxides, acts as a precursor in the production of other $2 \mathrm{D}$ structures or materials [113]. It displayed better activity in electrocatalysis, especially in the electro-oxidation of urea, hydrogen evolution reaction and oxygen evolution reaction; photocatalysis; and supercapacitors. It was observed that ultrathin LMHs showed improved catalytic performance because of the synergic effects of the hybrid interfaces [113].

Exfoliation and one-pot precipitation cannot be utilized for controlled production of distinct types of ultrathin layered metal hydroxides with covet structures. Moreover, when exfoliation crack phenomenon take place, it results in the formation of byproducts with lateral size of nanometers, while using inorganic precursors like anion intercalated bulk LDHs. By the method of bottom-up one-pot precipitation, layered single metal hydroxides such as cobalt(II) hydroxide and nickel(II) hydroxide can be formed. Intrinsic characteristics of the catalysts, which influence the activity of the catalyst, photochemical, and photo-electrochemical activity, are affected during the surface modification of the material owing to its extreme thinness [113]. Hybrids like plane-toplane heterostructures or van der Waals heterostructures, graphene-LMHs, and layered metal oxides displayed improved electrocatalytic and photocatalytic activity. Ultrathin layered metal hydroxides can also be obtained via partial conversion of layered metal hydroxides nanosheets to distinct species through oxidation, reduction, or substitution reactions [113].

2D nanoporous architecture method is utilized to increase edge active sites of ultrathin layered metal hydroxides, without altering crystallinity of the plane. As a result, the pore structures can be utilized as pathways for mass-transfer, 
in a direction perpendicular to the 2-dimensional planes. Furthermore, it can enhance the rate of ion transport between various layers of layered metal hydroxides in the electrode, thus ensuring the ion access the whole surface area [113].

The catalytic activity and performance of a silver-based catalyst depends upon its particle size of silver; the distribution pattern of atoms, type of clusters, and/or the fraction of nanoparticles of silver on the outer and subsurface layer, reactivity of functional groups containing oxygen formed over the surface of Ag; synergistic interaction between the components of the catalyst; and catalyst self-organization [114]. The activity of Ag containing catalysts can be increased by providing a proper support system, thus boosting the metal-support interaction (MSI) and by regulating the shape and size of silver particles. MSI over a $\mathrm{SiO}_{2}$ surface can be strengthened to obtain high dispersion state of silver particles by the following methods: (i) by conditional adsorption of $\mathrm{Ag}^{+}$particles on the surface of the support (when the $\mathrm{pH}$ is above 8 for $\mathrm{SiO}_{2}$ ) followed by the reduction into $\mathrm{Ag}$ nanoparticles through heterogenous nucleation; (ii) by using the organic derivatives of ammonia and carbamide for reduction, for functionalizing the active sites in one-pot synthesis; (iii) by the formation of silver nitrate particles with the help of isolate hydroxyl groups ( $\mathrm{OH}$ groups) through elevated temperature pretreatment that decompose to form defective multidomain silver nanoparticles which are active in low-temperature carbon monoxide oxidation [114].

The surface morphology of the transition metal oxides influences the Ag-support interaction. While using $\mathrm{MnO}_{x}$, the structural characteristics of the octahedral molecular sieves increase the rate of exchange of cation between silver and potassium. However, in cerium(IV) oxide catalysts, the reactivity is increased by the impregnation of $\mathrm{Ag}^{+}$ions into the inner layers of $\mathrm{Ce}$ through vacancies present in the oxygen atom [114]. During low-temperature oxidation of $\mathrm{CH}_{2} \mathrm{O}$, carbon monoxide, and $\mathrm{C}_{2} \mathrm{H}_{5} \mathrm{OH}$, the structural sensitivity between the various planes of Ce influences the creation of silver containing active sites. The transforming molecules get adsorbed onto the surface of the substrate during the cleavage of $\mathrm{C}-\mathrm{H}$ single bond in alcohols. The silver containing sites and acid-base sites of the support surface also helps in this adsorption process [114].

3.1.5. Material Morphology and Catalytic Consequences. $2 \mathrm{D} \mathrm{WO}_{3}$ nanomaterials are synthesized via hydrothermal, solvothermal methods, solid-liquid phase arc discharge (SLPAD) route, cold plasma method, and exfoliation techniques. $2 \mathrm{D} \mathrm{WO}_{3}$, when used as a supporting material, improved noble metals dispersion, interaction between the supporting material and the metal, and increased the electron density of platinum in the catalyst [115]. 2D $\mathrm{WO}_{3}$ nanomaterial finds application in photo-electrocatalysis processes, such as oxidation of water to oxygen, degradation of methanol using light/photon, and evolution of hydrogen through water splitting [115]. The direct synthesis route and indirect synthesis route of $2 \mathrm{D} \mathrm{WO}_{3}$ nanomaterials are given in Figure 4.

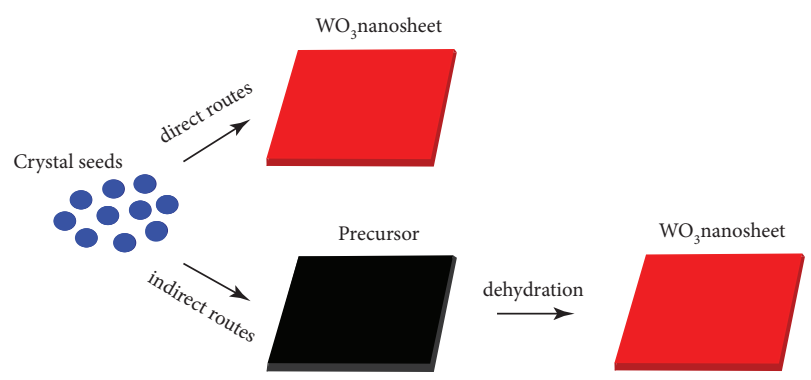

FIGURE 4: Direct and indirect synthesis route for 2D WO3 nanomaterials.

The photocatalytic activity of $2 \mathrm{D}^{-} \mathrm{WO}_{3}$, in $2 \mathrm{D}$ nanostructure, can be increased by decreasing the thickness of the material and size of the particle which resulted in an increment in the transferring rate of charge-carrier, lowering of $\mathrm{e}^{-}$-hole recombination of electrons and holes generated which were photogenerated, tuning of photoabsorption properties, and improved specific surface area. During solar energy conversion, the $2 \mathrm{D} \mathrm{WO}_{3}$ catalysts displayed high activity, optical characteristics, energy of bands, and greater charge-carrier transfer [115]. Additionally, $\mathrm{WO}_{3}$ nanosheets highlighted effective carbon monoxide oxidation rate, by changing the dispersion and electronic characteristics of the noble metal support and the interaction between the metal and the support. The surface electronic structure effect, owing to the difference in percentages of facets of $\mathrm{WO}_{3}$, affects the intrinsic absorption rate. Consequently, $\mathrm{WO}_{3}$ nanosheets helped in effective photoreduction of carbon dioxide molecules to methane compared $\mathrm{WO}_{3}$ catalyst with a cubic structure. $\mathrm{WO}_{3}$ act as a precursor in transition metal dichalcogenides $\left(\mathrm{WS}_{2}\right)$, with applications in Li-ion batteries and HER. In ultrathin monolayered $\mathrm{WS}_{2}$ nanosheets $\left(1 \mathrm{~T}-\mathrm{WS}_{2}\right)$, the strong interaction between the neighboring layers for restacking, deteriorates its electrochemical activity. As a result, $2 \mathrm{D} \mathrm{WO}_{3}$ nanomaterials having an ultrathin thickness is used as a precursor in the production process for ultrathin $\mathrm{WS}_{2}$ which possess a less restacking tendency [115]. Over the past few years, studies are conducted for the facile fabrication of monolayer $\mathrm{WO}_{3}$ materials to improve its semiconducting property to promote the absorption rate of visible light and photocatalytic performance.

The synergistic structural combination of noble metals and 3D-transition metals in the nanomaterials results in unique catalytic properties [116]. Due to the dynamic nature of $3 \mathrm{~d}$-transition metals, the activity of the catalyst in chemical/electrochemical reactions is supported by lattice strain of the structure and electronic/ligand effect. The highresolution TEM experiments done on each of the individual nanoparticles suggests the idea of "Pt-skin" in which the whole of 3D-transition is covered by noble metal shells [116]. However, in electrochemical reactions, a synergistic participation of 3D-transition metals on the active sites of the catalyst was observed, thus providing a stable alloy structure. Alloying-dealloying-realloying processes was done for dynamically evolve the structure of the catalyst. Furthermore, during dealloying, the dissolution of metal ion was facilitated by the presence of electrolyte at the interface of the catalyst. 
The core-shell/alloy dynamic structural changes were studied under oxidative environments for improving the performance of the catalyst in a gas-phase reaction, and thus to the design an active and stable catalysts [116]. It was observed that the oxygen-activation sites which were located on the outer regions of the noble metal catalyst with oxide support governed the gas-phase hydrocarbon oxidation reactions. By alloying the material using 3D-transition metals and noble metals, the activity of the adsorption sites carbon monoxide molecules was improved. Furthermore, surface-oxygenated 3D-transition metals offered additional oxygen-activation sites. Noble-metal/3D-transition metal alloy nanoparticles underwent surface oxygenation leading to the formation of composition homogenous alloy (CHA) type core with 3D-transition metal-oxygenated shell structure [116].

The function of $\mathrm{CuO}_{x} / \mathrm{CeOCeO}_{2}$ nanointerface in the catalytic oxidization of diesel soot was studied. $\mathrm{CeO}_{2}$ finds numerous applications in heterogeneous catalysis due to the ability of $\mathrm{CeO}_{2}$ to alter its ionic state between $\mathrm{Ce}^{4+}$ and $\mathrm{Ce}^{3+}$, thus creating oxygen vacancy defects. This is supported by the fact that the activation energy required for creating oxygen vacancies is less for transferring $\mathrm{Ce}^{4+}$ to $\mathrm{Ce}^{3+}$ over the $\{100\}$ facets/surface of cerium(IV) oxide than on nanoparticles [117]. The catalytic performance of $\mathrm{CeO}_{2}$ can be improved by the addition of transition metals to $\mathrm{CeO}_{2}$ nanocubes. Considerable number of oxygen vacancies are created by the dispersion of $\mathrm{CuOx}$, strong interaction with $\mathrm{CeO}_{2}$ and the facial coaction between $\mathrm{Cu}^{1+/ 2+}$ and $\mathrm{Ce}^{3+/ 4+}$ in $\mathrm{CuO}_{x} / \mathrm{CeO}_{2}$. The soot oxidation is promoted by the synergic effect at the nanointerface site in $\mathrm{CuO}_{x}-\mathrm{CeO}_{x} \cdot \mathrm{CeO}_{2}$ nanocubes are produced via template-free alkaline hydrothermal technique [117]. The $\mathrm{Cu}$ particle gets loaded on to the surface of $\mathrm{CeO}_{2}$ nanocubes via wet impregnation method. Both the catalytic and redox properties of $\mathrm{CuO}_{x} /$ $\mathrm{CeO}_{2}$ is supported by $\mathrm{CuO}_{x}$ and cerium(IV) oxide interaction along the edges of $\mathrm{CeO}_{2}$. The $\mathrm{CuO}_{x} / \mathrm{CeO}_{2}$ has a mesoporous structure. The percentage of $\mathrm{Ce}^{3+}$ ions $(10.98 \%)$ occurring to large amount of oxygen vacancies by transformation of $\mathrm{Ce}^{4+}$ to $\mathrm{Ce}^{3+}$, thus supporting its high catalytic reactivity [117]. $\mathrm{Ce}^{3+}$ ions facilitated the development of active species of oxygen at the $\mathrm{CuO}_{x}-\mathrm{CeO}_{2}$ interface, which can oxidize the soot as shown in Figure 5.

Ultrasmall niobium pentoxide nanomaterials having fluctuated structural motifs, acidity, and distortion act as an effective acid catalyst due to its unique structural properties and strong acid sites. Hydrated amorphous niobium pentoxides $\left(\mathrm{Nb}_{2} \mathrm{O}_{5} \cdot \mathrm{nH}_{2} \mathrm{O}\right.$, niobic acid) containing Lewis and Bronsted acid sites are utilized as a catalyst [118]. The ultrasmall nanoparticles of niobium pentoxide acid catalyst are produced via reacting $\mathrm{NbCl}_{5}$ with acetophenone, through aldol condensation, and results in the formation of hydroxylate niobium oxide nanoparticle $\left(\mathrm{Nb}_{2} \mathrm{O}_{5}-\mathrm{NP}\right)$, nanoparticles supported on holey graphene oxide $\left(\mathrm{Nb}_{2} \mathrm{O}_{5}-\mathrm{hGO}\right)$, and spherical arrangement of nanoparticles $\left(\mathrm{Nb}_{2} \mathrm{O}_{5}-\mathrm{NS}\right)$. High resolution transmission electron microscopy (HRTEM) indicated the existence of fringes in the lattice structure, suggesting a crystalline structure and defects in the structure of the nanomaterial, such as irregularities in the surface

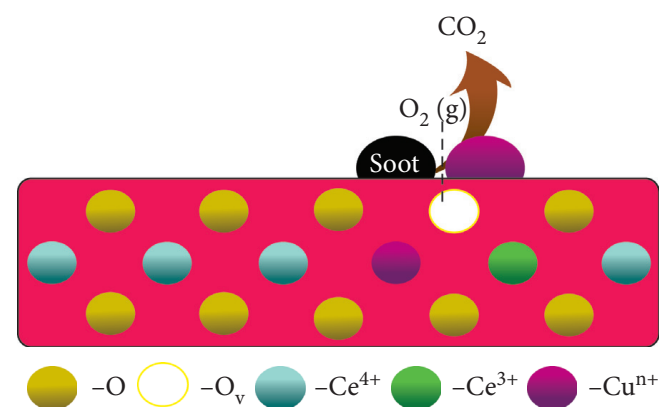

Figure 5: The oxidation of diesel soot over the interface of $\mathrm{CuO}_{x}-\mathrm{CeO}_{2}$.

boundaries and structural distortion. $\mathrm{Co}^{2+}$ ions used during the synthesis process (added during aldol condensation) expand the area available for reaction in nanoparticles, thus increasing its catalytic activity [118]. All the structures contained mesopores, with pore distribution size between $3-10 \mathrm{~nm}$. The ratio of number of Lewis acid sites to the number of Bronsted acid site was found out to be $\mathrm{Nb}_{2} \mathrm{O}_{5} \cdot \mathrm{nH}_{2} \mathrm{O}>\mathrm{Nb}_{2} \mathrm{O}_{5}-\mathrm{NS}>\mathrm{Nb}_{2} \mathrm{O}_{5}-\mathrm{NP}>\mathrm{Nb}_{2} \mathrm{O}_{5}-$ hGO. By spectroscopy, it was found out that $\mathrm{Nb}_{2} \mathrm{O}_{5}-\mathrm{NP}$ and $\mathrm{Nb}_{2} \mathrm{O}_{5}-\mathrm{NS}$ showed higher structural distortion than $\mathrm{Nb}_{2} \mathrm{O}_{5}-\mathrm{hGO}$ due the presence of substantial number of bridging hydroxyls in $\mathrm{Nb}_{2} \mathrm{O}_{5}-\mathrm{NP}$ and $\mathrm{Nb}_{2} \mathrm{O}_{5}-\mathrm{NS}$. By spectroscopy, $\mathrm{Nb}_{2} \mathrm{O}_{5}-\mathrm{hGO}$ was observed to have weaker acidity compared to other structures [118]. Ultrasmall niobium pentoxide nanomaterials showed better catalytic reactivity than $\mathrm{Nb}_{2} \mathrm{O}_{5} \cdot \mathrm{nH}_{2} \mathrm{O}$ in the conversion of hydroxymethylfurfural and in reaction between $\alpha$-angelica lactone and ethanol. $\mathrm{Nb}_{2} \mathrm{O}_{5}-\mathrm{NS}$ and $\mathrm{Nb}_{2} \mathrm{O}_{5}-\mathrm{NP}$ showed greater density of available acid sites, acidic strength and performance when compared to hydrated amorphous niobium pentoxides $\left(\mathrm{Nb}_{2} \mathrm{O}_{5} \cdot \mathrm{nH}_{2} \mathrm{O}\right)$. The synergetic adsorptive interaction between oxides and the carbon support is balanced out the weak acidity of $\mathrm{Nb}_{2} \mathrm{O}_{5}-\mathrm{hGO}$ in its catalytic activity [118].

Catalysts with varying inorganic morphologies and high catalytic reactivity are synthesized via peptide scaffolds based on the $\mathrm{Pd} /$ peptide ratio, including linear nanoribbons, nanoparticles, and complex nanoparticle networks [119]. Still, C-C coupling and 4-nitrophenol reduction were studied to understand the catalytic activity of bioinspired peptide-based systems. Because of large fraction of inorganic surface area and the depth of penetration within the scaffolds improved the catalytic reactivity of palladium nanoparticles and nanoparticle networks. The ligands present in the structure stabilized the catalyst along with improving its activity. Within these structures, the material aggregation is prevented by altering the surface layer of the material. By directly binding the ligands to the nanoparticle surface, the material stability is improved via steric effect or electrostatic repulsion. However, this could prevent the reactant from accessing the inorganic component, thus decreasing the reactivity [119]. The presence of bulky ligands such as peptides and dendron branching results in a steric bulk and surface binding mode, causing an improved exposure to inorganic components compared to a standard alkane thiol. High reactivity degree of the palladium particles supports 
several catalytic reactions, for example, carbon-carbon coupling and hydrogenation. The inorganic components of the catalyst are enclosed with in the organic framework of the catalyst, thus preventing bulk aggregation [119]. For example, the $\mathrm{Pd}^{2+}$ and $\mathrm{Pd}^{4+}$ ions are impregnated within the scaffolds to form a metallic nanomaterial through reduction. The exterior of the template provides high catalytic selectivity depending on polarity, composition, and size of the reagent. Having a complex morphology, these templates govern the rate of rection. During the reaction, the reagent should seep through the organic scaffold to react with the metallic part of the catalyst and pass in through the template and finally get released into the solution. Through templatebased synthesis, the depth of penetration of the template can be controlled along with the amount of surface area available for reaction, thus controlling its functionality [119].

Metal-based nanocatalysts have several chemical and industrial applications. However, due to the decrement in its strength at higher temperatures, it renders them unsuitable for processes involving higher thermal energy. Newly emerging nanocrystalline structures of high entropy alloys (HEA) showcases higher yield strength and durability to high-stress compared to nanocrystalline metals [120].

\subsection{Nanomaterials as a Catalyst in Fine Chemical Synthesis.} In this section, the scope of using the different types of nanomaterials and their applications as a nanocatalyst in fine chemical synthesis has been reviewed. The effect of functionalisation, modification with modifiers, promoters, dendrimers, and other such approaches have been mentioned. For the purpose of this review, nanomaterials have been divided into subdivisions (Figure 6).

3.2.1. Metal Nanomaterials. Modifiers are used to improve selectivity of catalysts. A common example of modifiers is "poisoned" Lindlar catalysts which lead to deactivate certain sites on the surface of palladium catalysts to prevent further hydrogenation, thus improving the selectivity. Li et al. successfully developed a more efficient replacement for "poisoned" Lindlar catalyst $\left(\mathrm{Pd} / \mathrm{CaCO}_{3}\right)$, in hydrogenation reactions of phenyacetylene and 3-phenyl-2-propyn-1-ol. They loaded palladium nanocubes onto $\mathrm{N}$-doped carbon nanofibrous microspheres (NCMs) to form Pd NCs@NCM which has an enhanced catalytic performance. Use of Mott-Schottky catalysts can be explored more deeply for metal nanomaterials in fine chemical synthesis [121].

Biaryls are produced by Suzuki-Miyaura cross-coupling reactions of organic halogen and organoboron using Palladium as catalyst. Their ability to bind multiple receptors is the reason why it is used in fine chemicals, which are complex, multifunctional molecules. This reaction also produces styrenes and alkenes. This reaction has a variety of applications in fine chemical synthesis, including synthesis of conjugated fluorescent polymers that remain stable even at $300^{\circ} \mathrm{C}$. They are used in sensors and organic light-emitting diodes (OLEDs) [122].

Jiao et al. carried out Suzuki-Miyaura coupling reaction of aryl bromide and chloride and phenylboronic acid. They used $\mathrm{NiO}$ nanodot-decorated $\mathrm{Pd} / \mathrm{SiC}$ as a catalyst which has Mott-Schottky contact which enabled efficient transfer of electrons to $\mathrm{SiC}$ from the palladium nanoparticles [122]. Aminophenols are very important in the fine chemical synthesis industry because they serve as intermediates for a number of fine chemicals, including dyes and pesticides. Pillararene can be used with gold nanoparticles catalyst supported by graphdiyne and covalent organic frameworks for the synthesis of aminophenol by reducing nitrophenol. Their catalytic performance was enhanced by the synergic effect between pillararene and the nanomaterials. The stabilized AuNPs displayed interesting sensing and self-assembly properties [123].

Mechanochemistry is a method or a protocol to synthesize highly active and stable catalytic nanomaterials [124]. Conventional mechanochemical methodologies like incipient wetness impregnation, co-precipitation among many others, can be sued for loading metal nanoparticles onto porous supports. Reactive milling can be used to make advanced bifunctional nanomolecular entities [125]. Nanoparticles tend to aggregate and supports like carbon nanotubes and grapheme, and mesoporous solid supports are used to stabilize them. Supports have a great influence on the catalytic performance, and if it gets modified suitably, it has the potential to behave as a cocatalyst [126].

Polyamine dendrimers are used to manipulate the morphology of metal nanoparticles. Dendrimer encapsulated nanoparticles are used to avoid agglomeration or precipitation and at the same time, provide accessible catalytic surfaces. Polyamine dendrimer with magnetite core that is encapsulated with gold nanoparticles was used to catalyze benzyl alcohol to form aldehyde. They showed good selectivity, stability, activity, and more active catalytic sites. This catalyst is reusable and does not lose its activity once reused [127].

MOFs exhibit properties such as superb selectivity, sensitivity, control, mass transfer and reaction rate improvement, and mechanical and chemical stability, making it very easy for the latter to be applied in copious areas of heterogenous organocatalysis. The ability to tune and control the morphological and the surface electronic properties make MOFs ideal. In general, the catalytic activity (based on size and dispersity) and stability (based on structure and interactive forces) of the supports are key constituents required for ideal support material. Impregnation, colloidal, and precipitation deposition are key methods for synthesizing single metal nanoparticles with MOFs as support [128]. MOF-derived materials provide a huge range of catalytic systems in the production of fine chemical. For example, MOF-derived cobalt-nickel alloy catalyst using N-doped carbon support (CoNi@NC800) are used in hydrosilylation of ketones [129].

It is interesting to note that metal catalysts can be used in colloidal form to avoid using supports, and it becomes easier to modify its morphology. Rhodium catalysts can be used in the colloidal form in hydrogenation reaction of anisole under mild conditions using a stabilizer [130]. The catalytic activity of colloidal metal catalysts relies on its method of production. However, the problem with colloidal catalyst is 


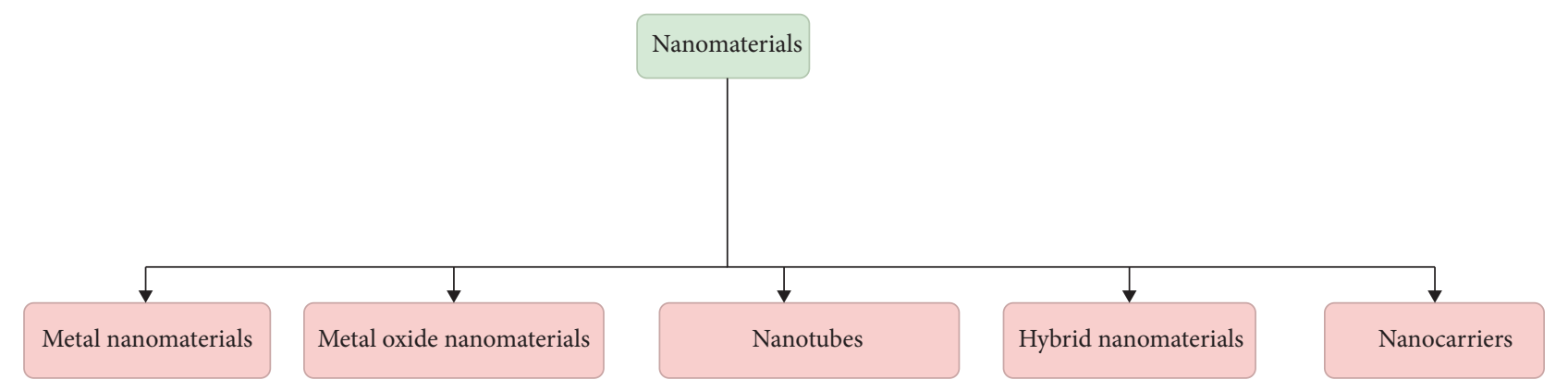

FIGURE 6: Demonstrates the classification of nanomaterials used in fine chemical synthesis.

quite obvious, it is difficult to separate them from the desired product and it is not stable. A thorough understanding of surface and the morphology is needed in order to design catalysts. Factors affecting the performance of heterogeneous catalysts include [9] the effect of metal loading introduced, use of supports, modifiers and promoters. Organic ligands are one of the stabilizers used for nanoparticles [131]. Organic ligands are observed to have adverse effect on the colloidal metal catalysts. They make certain sites on the surface of the metal inaccessible. Thus, a number of attempts have been made to remove these ligands. However, it has been found out that they can be tuned to enhance the catalytic activity and selectivity, which are otherwise highly responsive to the structure of organic species.

Organic and inorganic modifiers have been used to tune catalyst properties. Organic compounds reduce the free energy of between the metal and metal oxide surfaces and their environment which is why they adsorb these organic compounds on their surfaces (Figure 7). These organic compounds can also alter the interfacial surfaces and improve their stability and prevent aggregation. Surfactants are used as stabilizers because of their amphiphilic monolayers. Self-assembled monolayer (SAM) is an assembly of organic molecules with a headgroup which interacts with a solid substrate to form a stable monolayer film, and a tailgroup which can be tuned to alter the properties of a catalyst (Figure 8) [131, 132]. SAM can be used to increase selectivity or specificity in heterogeneous catalysts by tuning their properties by tuning their active sites or the interaction between the molecules of modifiers and reactants and steric effects. Chen and Rodionov [126] reported the use of bimetallic alloy nanomaterials modified with ligands and polymers, in controlling the activity and selectivity of catalysts.

3.2.2. Metal Oxides. Doped $\mathrm{TiO}_{2}$ nanomaterials were used as biocidal agents and water and soil remediation. Zinc oxide simultaneously senses and degrades organic contaminants in water such as 4 -chlorocatechol [133]. $\alpha$-Silyloxy- $\gamma$-indolyl nitriles were synthesised by employing $\mathrm{InBr} 3$ as a catalyst in Michael 1,4-addition of indoles to $\alpha, \beta$-unsaturated ketones and succeeding 1,2-addition of trimethylsilyl cyanide [134]. Kantam et al. replaced $\mathrm{InBr} 3$ by nanocrystalline $\mathrm{TiO}_{2}$ which resulted in complete conversion with $54-73 \%$ yield within 10 hours [135].

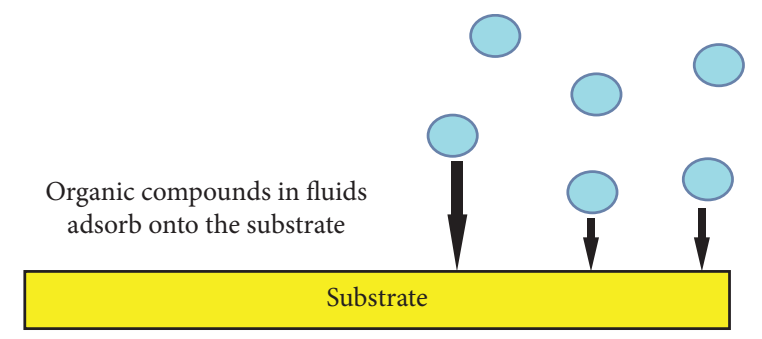

Figure 7: The substrate is the metal or the metal oxide which adsorbs the organic compound into their surface. A self-assembled monolayer is formed due to this interaction.

Mahmoud et al. made nanosorbents to eliminate $\mathrm{Cu}(\mathrm{II})$ and $\mathrm{Pb}(\mathrm{II})$ heavy metals from water which was synthesized via functionalization of (nano- $\mathrm{Fe}_{3} \mathrm{O}_{4}-\mathrm{SiO}_{2}$ ) sorbent with triethylenetetramine [136].

Cupric oxide has been reported to exhibit antioxidant and antibacterial activity [137]. Biopolymer-based nanomaterials are used in anticancer and antimicrobial drugs. Karthikeyan et al. designed an environmentally benign synthesis method for producing chitosan-copper oxide nanomaterial which was more active than amoxicillin against bacteria [138]. The material also showed anticancer cytotoxic activity targeting cervical cancer cells.

$\mathrm{N}$-doped mesoporous carbon has been reported to be efficient supports for metal nanomaterials because MottSchottky effect and also the uniformity of their highly accessible, porous, and heterogeneous sites which can be tuned easily [139-141]. Li et al. [132] reported the use of magneticdoped graphene support for $\mathrm{Co}_{3} \mathrm{O}_{4}$ nanocatalyst in alkene epoxidation reaction was more efficient than nonsupported and unreduced nanocatalyst, with $93.2 \%$ conversion rate and $88.4 \%$ epoxide selectivity. The catalyst could be reused by retrieving them using a permanent magnet.

Spirooxindoles have antidiabetic, antimicrobial, and antitumor properties. They are used in the pharmaceutical industry as precursors for drugs [142]. Moradi and Ataei synthesized high yield of spirooxindoles via a green one-pot process by employing copper oxide nanoparticles as catalyst at mild conditions. This low-cost catalyst could be reused, retrievable, and reduced the reaction time significantly [143]. Karmakar et al. synthesized fused spirooxindoles using nanocrystalline $\mathrm{MgO}$ as catalysts [144]. Spirooxindoles fused with isatin have medicinal applications [134, 142, 145, 146]. Tameh et al. synthesized spirooxindoles via a one-pot 


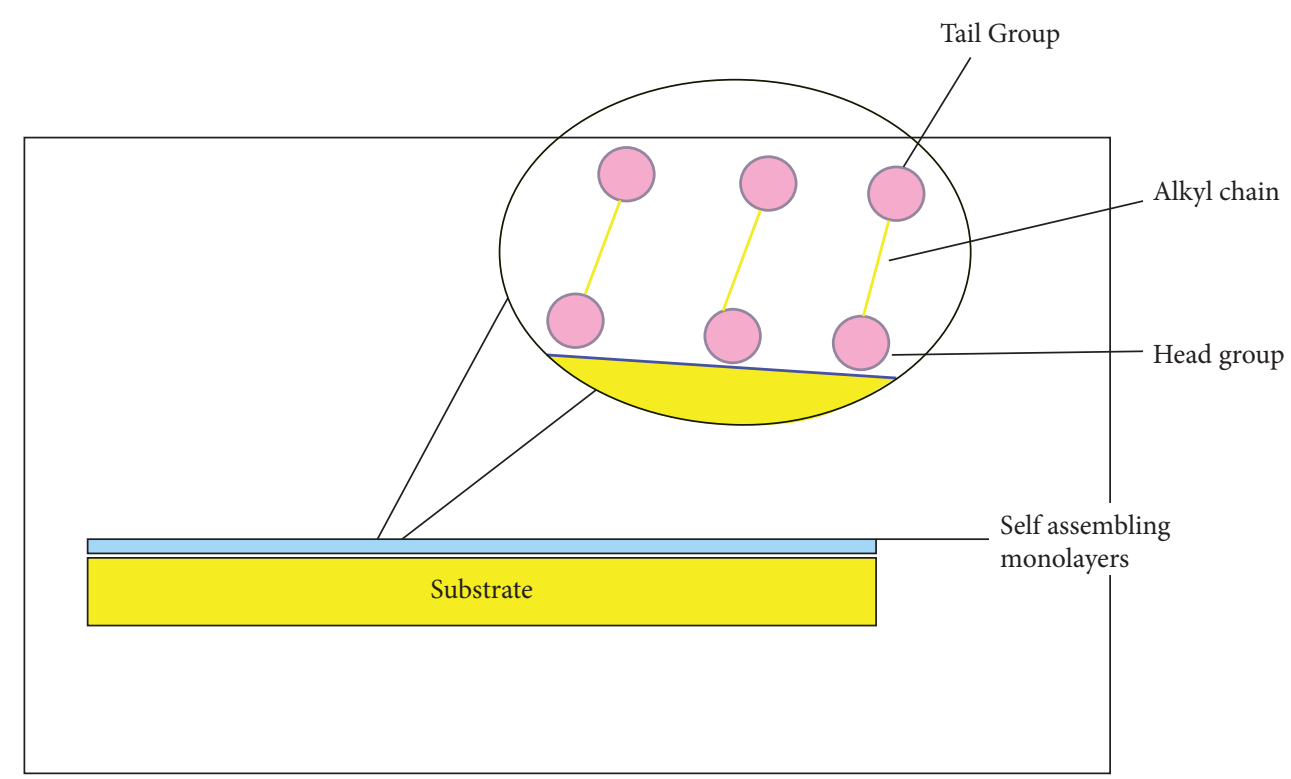

FIGURE 8: Self-assembled monolayer (SAM) is an ordered assembly of organic molecules with a headgroup which interacts with a solid substrate to form a stable monolayer film and a tuneable tailgroup.

catalytic reaction using magnetic iron nanoparticles functionalized by guanidine [147]. Guanidines are stronger organic bases than the other nitrogen-based compounds. Application of magnetic metal nanoparticles in the reaction made the separation process easy and green since the catalyst can be recycled after subjection to magnetic field. Surface functionalization of magnetic nanoparticles has been gaining a lot of attention. Enzymes, transition metal catalysts, bioactive polymers, and organocatalysts can be immobilized on magnetic nanoparticles [148]. Sumit et al. synthesized an efficient method to synthesize zinc ferritesulphated nanoparticles which is used as a catalyst in fine chemical synthesis [149].

Chiral ligand-modified metal nanoparticle has a lot of scope in asymmetric synthesis. Wu et al. [131] used modified iron oxide as a magnetic catalyst for hydrogenation asymmetric reaction. The reaction had good enantioselectivity and reusability up to 5 cycles, but their activity was low. Metal ligand systems are preferred over supramolecular catalysts because they are more cost efficient and have better enantioselectivities. However, using multiple supramolecular systems has a potential of enhancing catalytic activity and selectivity and cut down cost due to potential of reduced amount of catalyst required for the reactions [150].

3.2.3. Nanotubes. Metal-free carbon nanomaterials are widely accepted because they are easily accessible, environmentally benign, resistant to corrosion, and possess exclusive surface properties. They include fullerene, carbon nanotubes (CNTS), and nanodiamonds graphene sheets. Classification of CNTs based on the number of graphene sheets present is shown in Figure 9. Carbon nanomaterials can be divided, based on their confinement, into four categories: zero-dimensional (spherical or nearly spherical shapes), one-dimensional (long length nanowire
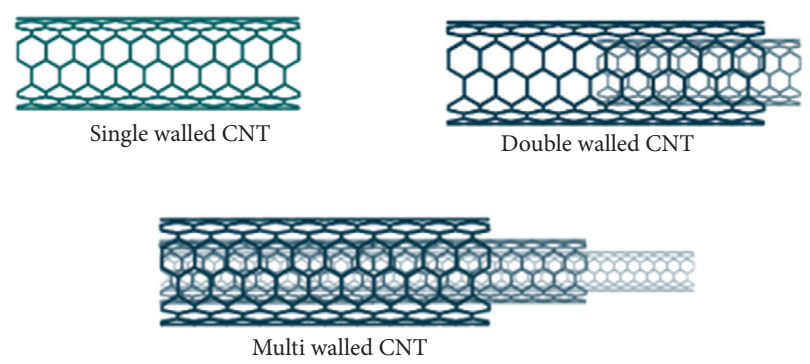

FIGURE 9: Classification of CNT based on the number of graphene sheets.

or nanotubes), two-dimensional (well aligned sheet-like structure called nanosheets or nanoplates), and threedimensional (3D). Each dimension has its impact and advantages [151]. Metal catalysts supported by carbon nanomaterials have higher surface area, stability, and lesser tendency to allow immobilized metal particles accumulation than carbon nanotubes. The catalytic activity of CNTs depends on its diameter and the applied metal deposition method used in them [107].

Carbonaceous materials have large BET surface areas, are stable, and have excellent corrosion resistance. Their hydrophobic and wettability property make them suitable for acid site functionalization. Graphene and CNTs are thermally and mechanically stable and have better hydrophobicity than the other amorphous carbonaceous materials [152]. Doping nanostructured carbon materials with heteroatoms increases reactivity. Graphene flakes and MWCNT doped with heteroatoms can be used to reduce 4-Nitrophenol. Doped materials are active catalysts with good efficiency in reducing 4-nitrophenol in the presence of $\mathrm{NaBH} 4$. As opposed to the pristine materials, the materials refined by doping with heteroatoms yield excellent catalytic activity [153]. 
3.2.4. Hybrid Nanoparticles. Organization nanoparticles with a complex structure and coordination polymers are the most common examples of hybrid nanomaterials. In this review, the use of hybrid nanoparticles in environmental remediation and drug synthesis is highlighted.

Usually, the properties of a material are enhanced when used in conjunction with another material. Metal nanoparticles achieve stabilization and increased catalytic performance when combined matrices are used. Hybrid nanoparticles can be a composition of only organic compounds, only inorganic compounds, or a combination of organic and inorganic compounds. The possibilities of combination of these compounds are endless, and hence, there is a lot of potential for this nanomaterial to be used in fine chemical synthesis. The properties of the resulting hybrid nanoparticle can be synergized, or they can be an average of the properties of that component or an additional property may be provided from one component to the other. Quantum size effect results from the extremely nanosize of the nanoparticles which gives them special properties. The surface atoms of the nanomaterials are predominant because of its large surface area and small size. Core-shell systems can be used in pharmaceutical industry for drug delivery, when it is enclosed in a porous matrix, and in catalysis in drug synthesis. There are a lot of studies revolving around organic-inorganic hybrid nanomaterials [154].

Carbon black can be used to support nickel-metal nanoparticles, which have good catalytic activity, chemical stability, and conductivity. This hybrid C-Ni nanocomposite is used for efficient hydrogenation of 2-nitrophenol to form 2-aminophenol under mild conditions [155]. Graphenebased composites can be used in photocatalytic selective oxidation in fine chemicals synthesis for sustainable and efficient exploitation of available feedstocks [156].

Metal organic frameworks modified with chiral ligands have good stability, accessibility of active sites and porous structure [157]. Chirally modified MOFs have been researched as a catalyst in asymmetric synthesis [158-161] for the past twenty years and synthesis methods with selectivity and enantioselectivity as high as $99 \%$ have been developed. Sayed et al. [162] synthesized an efficient chiral hybrid nanomaterial with metal-organic framework which had selectivity and enantioselectivity of $99 \%$ in nitroaldol condensation reaction. They are still working on this strategy and trying to improve it since the pores did not accommodate the asymmetric Henry condensation. The pores of MOFs can successfully incorporate multiple distinct active sites which makes it an attractive support for one-pot synthesis. There should be further research to implement asymmetric catalytic synthesis by MOFs modified with chiral ligands in practical applications along with working on battling their drawbacks such as leaching, improving their TON and stability, increasing their pore size and finally, and improving the versatility of their applications. This approach has a high scope in fine chemical synthesis because of the possibility of high enantioselectivities achieved [159].

Biomass-derived substrates such as chitosan and starch and cellulose have been used to prepare nanomaterials. This method of nanocatalyst production is environmentally benign and complied to the green engineering principle. Chitosan has also been used as a biopolymer to produce biocide formulations having $\mathrm{ZnO}$-interlinked chitosan nanoparticles [43].

Inorganic nanoparticles substituted with natural organic biopolymer are of low cost, stable with no particle agglomeration, the toxicity of metal ions is lower, and are reusable. Nanomaterials having more oxygen vacancies with good photocatalytic activity induced more generation of ROS (singlet oxygen and hydroxide radicals) molecules destroyed cells walls of the bacteria by exertion of oxidative stress, killing them. CuO NPs generate ROS and inactivate the bacterial cells proteins by binding $\mathrm{Cu} 2+$ ions to the bacterial cell surface. They are used to kill E. coli by sol-gel and biosynthesis methods. CuO-curcumin used with ultrasonication method is used to kill $E$. coli and S. dysenteniae. CuO-GO-Ag can be used to kill E. coli using chemical bath deposition methodology. rGO-CuO NPs can transfer electrons from the cells of $P$. aeruginosa, leading to their inactivation [138].

Attia et al. used $\mathrm{Ag} / \mathrm{ZnO} /$ graphene-Si hybrid nanocatalyst in green synthesis of nictonic acid (vitamin B3). This method is an alternative, environmentally benign approach to make the use of corrosive chromic acid redundant as an oxidant in the fine chemical industry [163]. Nafion resin is highly acidic but has a small surface area which leads to lower activity. Hinze et al. synthesised a highly stable and active Nafion/silica composite with nanosized Nafion particles enclosed in a silica matrix. This nanocomposite can be used in fine chemical synthesis for production of paracetamol and vitamin E [164].

3.2.5. Nanocarriers. Nanocarriers have significant application in pharmaceutical industry in drug targeting and drug delivery. They make use of the advanced characteristics of nanomaterials which can be tuned and functionalised to encapsulate important drugs and enhance their efficiency. Their extremely small size and interfacial properties ensure increased bioavailability of the drug in the body. The classification of nanocarrier has been illustrated (Figure 10). The use of nanocarriers in drug delivery along with some of their applications will be explained in this section. Nanocarriers provide the following advantages in drug delivery:

(1) They increase the bioavailability of the drug since the level of absorption of the drugs by the tissues is increased

(2) They improve drug stability by protecting moieties from chemical and light degradation

(3) They can regulate the release of the drug into the body

(4) They prevent the drug from trans-cis isomerization

(1) Vesicular Nanocarrier. Phospholipids are amphipathic compounds that have the ability to form bilayer membranes in aqueous medium because of their hydrophilic head and hydrophobic tails that cause them to fold into closed 


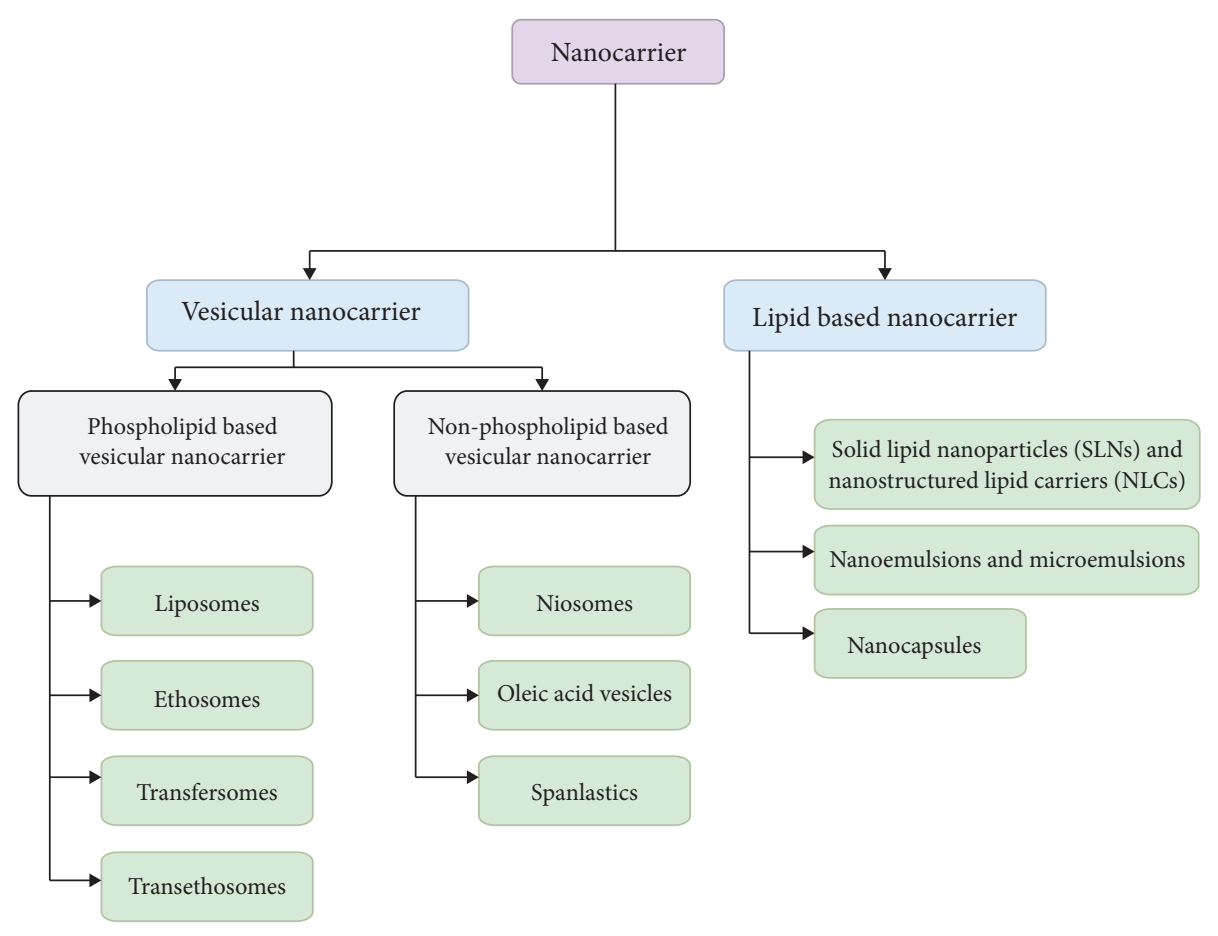

Figure 10: Classification of nanocarriers.

concentric vesicles to make the structure stable and reduce the surface tension. They have emulsifying and wetting properties and can self-assemble and form different structures at specific environmental conditions. These properties make them eligible to be used in the drug delivery system. They can be used to coat hydrophobic drugs to increase their hydrophilic property of the drug [164]. They have been used in drugs such as doxil, cleviprex, valium, and silybin phytosome $[165,166]$. Lipid-based nanoparticles such as solid lipid nanoparticles (SLNs), nanostructured lipid carriers (NLCs), nanoemulsions and microemulsions, and nanocapsules can be used in drug delivery for encapsulating hydrophobic drugs [167].

(a) Liposomes

Liposomes can be used to encapsulate a wide variety of drugs (Table 7) by passive or active drug loading [177]. They are vesicular structures where an aqueous core is enclosed by a membrane of phospholipid bilayers which form when they are dispersed and exposed to water. These biocompatible systems can enclose lipophilic and hydrophilic compounds as demonstrated (Figure 11).

The cancer cells require fat for the cell growth and perceive liposomes as a good source of fat. They get absorbed into the liposome and get exposed to the anticancer drugs and get killed (Figure 12). Liposomes can be used to encapsulate cytosine arabinoside, a chemotherapy drug used to cure acute myeloid leukemia to increase its bioavailability [178]. (b) Niosomes

These nonphospholipids based vesicular nanocarriers are used in drug delivery and enhances the efficacy of the drug they encapsulate. They form by self-assembly when nonionic surfactants are introduced in the formation of liposomes. The aqueous core entraps the hydrophilic drugs and the lipophilic drugs are loaded on the bilayer membrane of the nanocarrier. Their effect on certain drugs is demonstrated (Table 8).

\section{(2) Lipid-Based Nanoparticles}

(1) Solid lipid nanoparticles (SLNs) and nanostructured lipid carriers (NLCs)

(2) Nanoemulsions and microemulsions

(3) Nanocapsules

Lipid-based nanoparticles are used for encapsulating hydrophobic drugs and improve drug transport and aborption in the gut for lipophilic compounds [167]. They are being used used to encapsulate Pfizer and Moderna COVID-19 mRNA vaccine, (Figure 13). The mRNA containing spike proteins is optimised to be used in the vaccine. It is then injected into the body where it comes in contact with human cell and released mRNA into the cell, ordering it to produce spike proteins. The immune system fights against these spike proteins by producing antibodies. At physiological $\mathrm{pH}$ range, lipid-based nanocarriers have a neutral charge on the lipids, eliminating any toxic side effect of using these nanocarriers which is otherwise associated with positive charge of liposomes. They are also used in patisiran drug to carry nucleic acid. 
TABLE 7: Effects of encapsulation of the drug by liposome on its activity.

\begin{tabular}{|c|c|c|c|}
\hline $\begin{array}{l}\text { Sr } \\
\text { no. }\end{array}$ & Drug encapsulated by liposome & Effect & Reference \\
\hline 1 & Resveratrol & $\begin{array}{l}\text { Liposomal incorporation was found to make resveratrol more efficient in } \\
\text { treating the oxidative damage caused by UV-B on skin }\end{array}$ & [168] \\
\hline 2 & Polymixin B & $\begin{array}{c}\text { Improved the efficiency in reducing Pseudomonas aeroginosa bacteria count } \\
\text { in lung infection }\end{array}$ & [169] \\
\hline 3 & $\begin{array}{l}\text { N-methyl-N-D-fructosyl amphotericin B } \\
\text { methyl ester (MFAME) }\end{array}$ & Enchanced antifungal activity and reduction of toxicity & {$[170]$} \\
\hline 4 & Doxorubicin & Enchanced antitumor activity & [171] \\
\hline 5 & Gentamycin & Enhanced the efficiency of the drug & [172] \\
\hline 6 & Paclitaxel & Improved the solubility of the drug & [173] \\
\hline 7 & Topotecan $\mathrm{HCl}$ & Stabilised and improved the efficiency of the anticancer drug & [174] \\
\hline 8 & Ketaconazole & Decreased the side effects of the drug along with increasing its efficacy & [175] \\
\hline 9 & Hydroxyzine & Improved efficiency of the antihistamine sedative drug & [176] \\
\hline
\end{tabular}

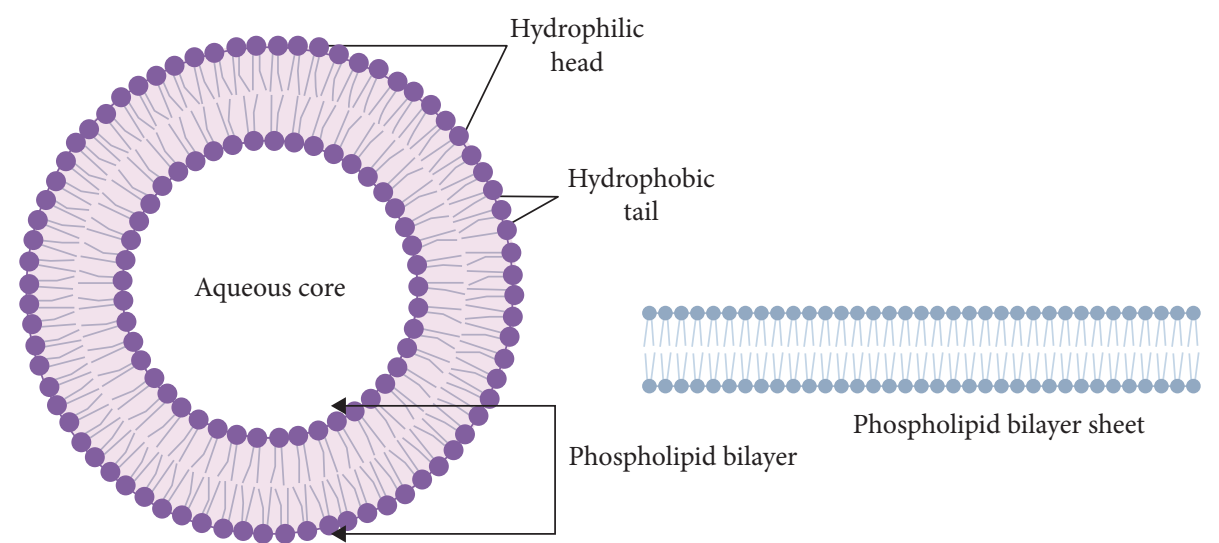

Figure 11: Structure of liposome and its phospholipid bilayer sheet.

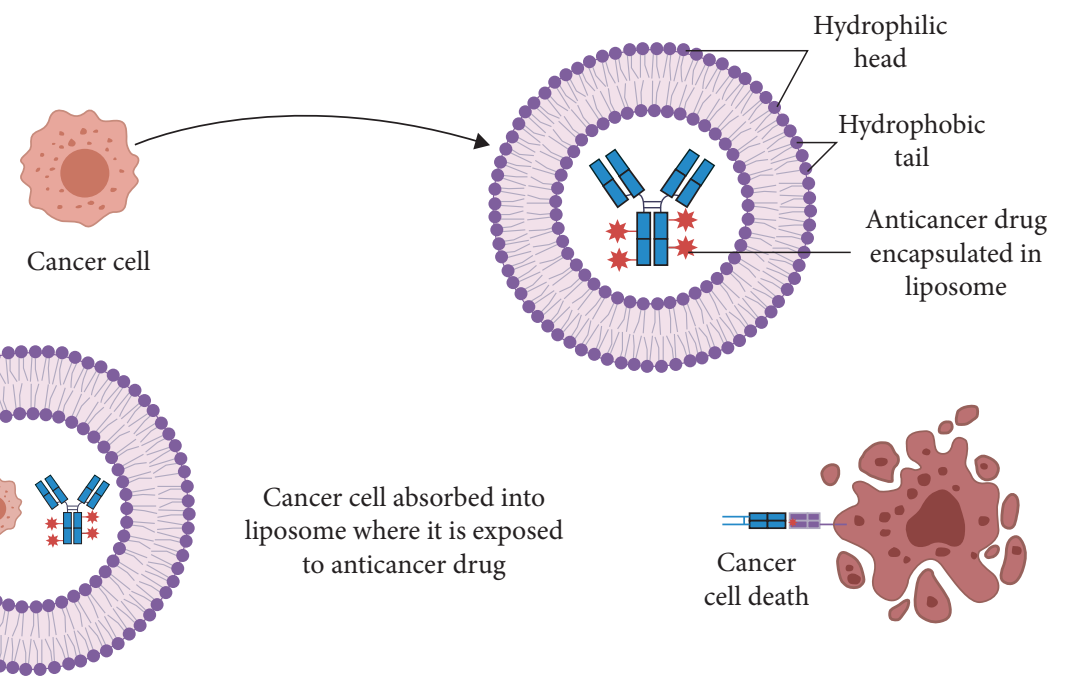

FIGURE 12: Illustration of working of a liposome encapsulating anticancer drug against cancer cell.

TABLE 8: Effects of encapsulation of the drug by niosomes on its activity.

\begin{tabular}{cccc}
\hline Sr no. Drug encapsulated by Niosomes & Effect & References \\
\hline Artemisone & Increased the selectivity to kill melanoma cells without harming the skin cells & {$[179]$} \\
5-Fluorouracil & Improved the efficiency of the drug & {$[180]$} \\
Cisplatin & Decreased the toxic effects of toxic cisplatin and improved the antimetastatic activity & {$[181]$} \\
Adriamycin & Prolonged the lung tumour growth & {$[182]$} \\
Pentoxifylline & Improved the efficiency of tumour reduction in the lung & {$[183]$} \\
\hline
\end{tabular}


3.2.6. One-Pot Reactions in Fine Chemical Synthesis. Catalysts are used to increase the molecular interactions between the reactants and hence enhance the rate of reaction by reducing the energy of activation. Homogeneous catalysts have same phase as the reactants so the interaction between the catalyst and the reactant is higher than in heterogeneous catalysts, but retrieving these catalysts is difficult and costly techniques such as nanofiltration and column chromatography separation need to be used [184].

There are a lot of reaction steps involved in fine chemical synthesis which can be reduced using multifunctional catalysts in one-pot synthesis, which also improves atom economy. Selective reactions with economical and maximum conversion of reactant atom to product atom are required in fine chemical synthesis. Transition metals like palladium and gold have good selectivity and conversion rate, especially in synthesis of cyclic structures, as a result of their large, highly specific surface area which contain a lot of active sites [126].

Nickel carbonyl is toxic which is why stable palladium is used instead of nickel catalysts in cross-coupling reactions, even though their reactivity is lower. A major breakthrough in the organic synthesis industry was the usage of palladium catalyst in cross-coupling reactions, which can be in the form of complexes comprising ligands in organic solvent and are generally homogeneously catalysed [185].

Palladium is a transition metal with incompletely filled d-orbitals which can bond with multiple functional groups, which is why they are used in cross-coupling reactions to mediate the reaction between aryl halides, an organic electrophile, and an organic nucleophile [186]. Some of the applications of palladium as a catalyst in fine chemical synthesis are listed in Table 9.

Pdo is used as a catalyst in Suzuki-Miyaura, where the reactants are aryl halide and organoboron, and Sonogashira reactions. Aidan et al. synthesised supported Pd0 nanoparticles by exposing $\mathrm{Pd}^{2+}$ to alkenes [201]. Homogenous palladium catalysts use N-heterocyclic or even phosphine ligands for catalysing Heck and Suzuki reactions. Oral concentration of residual palladium in pharmaceutical substances should not be more than $5 \mathrm{ppm}$. The purification process for bringing the concentration to the acceptable limits is expensive and time-consuming, which is why using reusable heterogeneous catalyst is preferred [184].

$\mathrm{Pd} / \mathrm{C}$, one of the first solid catalysts to be used, has thirtysix thousand TON value in Suzuki, Sonogashira, and Heck coupling reactions. Jadhav et al. dispersed $\mathrm{Pd} / \mathrm{C}$ in a hydrotropic medium to stabilise them for its application in Suzuki and Heck cross-coupling reactions [202]. The reaction was carried out without toxic organic solvents, had a good yield, and was reusable thrice without losing its activity. In addition to all of this, there was no leaching observed [184].

Negative interactions between multiple homogeneous organometallic catalysts used in the same system reduce their catalytic activity because of the difference in their coordination chemistry [203]. Supported liquid-phase catalyst (SLPC) is used in organic reactions to make the catalytic reactions recyclable and reusable due to ease of separation of catalyst from the reactants. The support contains the organometallic complex in a hydrophilic liquid film. A hydrophobic solvent is used to contain the SLPC. Multiple SLPC systems can be separated by the hydrophobic solvent, and metal leaching is successfully circumvented [204]. Application of SLPC in fine chemical synthesis has the potential to increase since it significantly reduces the steps involved in the reaction and is a greener option. Dibenzyls are used in the pharmaceutical industry in lunularic acid and bifluranol. A number of experiments which have been conducted in an attempt to optimize the synthesis of Dibenzyls are mentioned as follows.

Bhanage et al. used SLPC for a bifunctional catalytic system of palladium and rhodium for concurrent $\mathrm{C}=\mathrm{O}$ and $\mathrm{C}=\mathrm{C}$ bond hydrogenation and heck reaction followed by hydroformylation reaction to produce styrene which is then converted to 2-phenylpropionaldehyde, which is used in synthesis of naproxen and ibuprofen. Yield in a system composed of (palladium + rhodium) SLPC was lower than that of a mixture consisting of palladium-SLPC and rhodium-SLPC. They demonstrated that using SLPC, two homogeneous catalysts can maintain their catalytic performance with reduced negative interactions [205]. Two shortcomings of this system is the need for the organometallic complexes to be supported and the performance of SLPC is not enhanced, and it stays the same as the individual activity of the catalyst. For this reason, homogeneous catalysts in one-pot synthesis must be carried out using only one catalyst which can catalyze the entire series of reactions solitarily since use of more than one homogeneous catalyst results in negative mutual interactions causing reduced catalytic activity [203].

Heterogeneous catalysts have a different phase than the reactants and have binding sites on their surface which adsorb the reactants. The performance of these catalysts is determined by the availability of the binding sites because the reaction can only continue after the products are removed from the binding site. Filtration can be used to separate the catalyst from the product so that precious metal catalysts can be reused. Their use in one-pot reactions is demonstrated in the following experiments. Reusing expensive metals and reducing the waste and metal contamination in the resultant product is very important for a sustainable and green catalytic organic synthetizes which can be achieved using heterogeneous catalysts.

Organic and inorganic supports are used for making palladium catalysts more efficient, reusable, and easy to separate from the products in coupling reactions. Inorganic supports include zeolites, alumina, and silica. The problem with using these supports is reduced activity due to poor molecular interaction among the reactants and the active sites on supports.

Some factors reducing yields in palladium catalysts with supports are mentioned. Palladium nanoparticles tend to aggregate and leaching occurs after a number of cycles, reducing surface area of the catalyst. Active sites are also damaged and blocked by poisoning and accumulation of salt, reducing the amount of palladium available to catalyze the reaction [206]. 

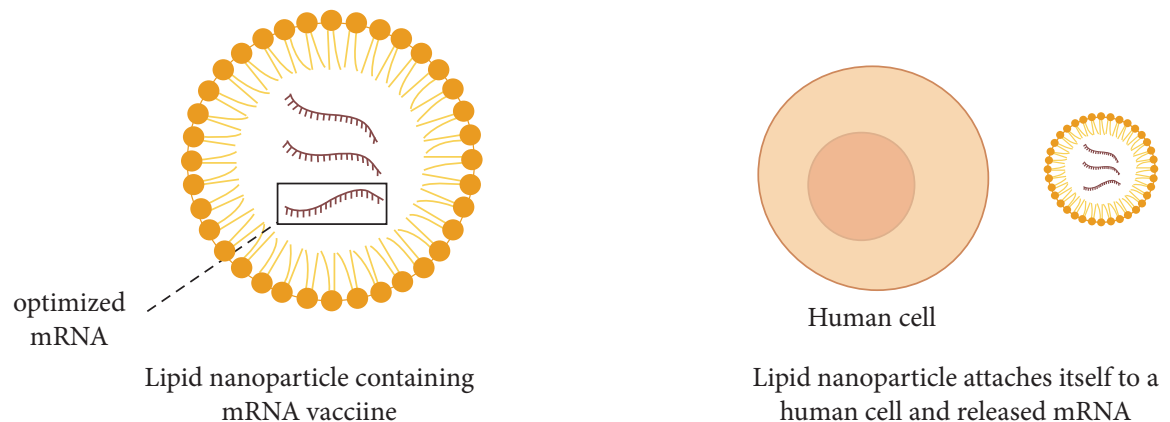

FIGURE 13: Illustration of COVID-19 antibody containing lipid nanoparticle containing the mRNA.

TABLE 9: Synthesis methods using palladium as catalysts.

\begin{tabular}{lccc}
\hline Sr. no & Reaction & Application in fine chemical synthesis & Reference \\
\hline 1 & Heck coupling reaction & Dibenzyl synthesis & {$[187]$} \\
2 & Heck coupling reaction & 2-Styryl-phenylamine synthesis & Wadsworth-Emmons reagents \\
3 & Heck coupling reaction & 4-Styryl-biphenyl & {$[187]$} \\
4 & Heck and Suzuki coupling reaction & Terphenyls & {$[188]$} \\
5 & Double Suzuki cross-coupling reactions & Oxindoles & [190] \\
6 & Heck coupling reaction & 2-Quinolones & {$[191,192]$} \\
7 & Heck coupling reaction & Asymmetrical ethers synthesis & {$[191]$} \\
8 & Acetalization followed by hydrogenolysis & Asymmetric linear ketones with $\alpha$-stereogenic center synthesis \\
9 & Asymmetric tautomerization & Imines and secondary amines & {$[194]$} \\
10 & Oxidation-condensation & Chiral diols & {$[195]$} \\
11 & Asymetric diethylzinc addition & Imines & {$[196]$} \\
12 & Oxidation-condensation & Quinoxalines & {$[197]$} \\
13 & Oxidation-condensation & Isopulegol epoxide & {$[198]$} \\
14 & Cyclization-epoxidation: & N-acylated-amino alcohols & {$[200]$} \\
15 & Hydrogenation & &
\end{tabular}

Lamblin et al. reported that, after a number of reuses of heterogeneous catalysts supported on active carbon and polyaniline, they will start to deactivate due to leaching and degradation of the support [207]. Carbon nanotubes, graphenes, chitosan, and dendrimers act as a support for palladium in cross-coupling reactions [186]. Activated carbon leads to catalyst poisoning and stability issues [208]. So, better alternatives to this must be found.

Among all the carbon materials, CNTs are known to have better thermal and electrical conductivity. The molecular interactions between the reactant and the palladium supported on carbon nanotubes are high due to the small size of CNTs which increases the availability of pores to the reactants. Using CNTs as supports increases molecular interactions since they have smaller size and can be uniformly dispersed in the solution. They are divided into single-walled carbon nanotubes (SWCNTs), double-walled carbon nanotubes (DWCNTs), and multi-walled carbon nanotubes (MWCNTs). CNTs have good yields and conversion rates, but their activity reduces after a number of cycles due to leaching and poisoning of their active sites. Labulo et al. [185] reviewed techniques for functionalizing the surface of CNTs in an attempt to make it a better catalyst support.

Surface functionalization increases binding sites and surface anchor groups and increases the surface area by addition of functional groups to improve the deposition of the nanomaterial on the surface. Thus, the molecular interactions between the active phase and the support are improved, and its properties are tuned [185]. Surface oxidation is a covalent method of functionalization of CNT in which the functional group with oxygen like phenols is formed on the CNT surface. The methods used for functionalization, like wet chemical methods or gas phase methods, also help in purifying and can be used to cut CNTs [209]. Surface halogenation can be performed for functionalizing MWCNT with halogen, and the resulting halogenated CNT exhibits photoluminescence [210]. Chlorinated and brominated CNTs cannot be used in substitution reactions [211], and fluorinated CNTs can be used as insulators. Noncovalent functionalization preserves the electrical properties of the CNTs unlike covalent functionalization [212]. CNTs can be doped with heteroatoms to improve its stability and activity. Li et al. reported use of nitrogen-doped CNT support with palladium nanoparticles for the one-pot synthesis of pyrazole [104]. N-doped MWCNTs have been used for coupling reactions where palladium leaching did not take place, number of active sites increased, and the catalytic performance was improved [213].

ike CNTs, graphene oxides have been reported to have high surface areas and excellent electrical properties which is why it has been used as a support in fine chemical synthesis. 
Rana et al. [214] used a diamine to functionalise the surface graphene oxide to be used as a catalyst for one-pot synthesis of pyrazolo-pyranopyrimidine derivatives which can be used as scaffolds. The reaction was successful with good yield and selectivity.

Lakshminarayana et al. [215] synthesised PdO nanoparticles supported on a variety of carbon-based nanostructures such as carbon nanofiber, SWCNT, MWCNT, graphene oxide, and reduced graphene oxide. They examined their effect as a support in Heck olefination of aryl halides and concluded that $\mathrm{PdO}$ supported by graphene oxide, $\mathrm{PdO} / \mathrm{GO}$ outperformed the other characterised supports with its high performance. Gruber et al. [187] attempted to use palladium catalyst supported on activated carbon, $\mathrm{Pd} / \mathrm{C}$, to synthesise dibenzyl in a one-pot reaction.

Kantam et al. reported that using a palladium catalyst with nanocrytalline magnesium oxide (NAPMgO-Pd) in a one-pot synthesis for synthesis of dibenzyls prove to be the most efficient catalyst among other palladium catalysts based on activated carbon, silicon dioxide, titanium dioxide, and aluminium oxide. This catalyst was reusable and did not lose its stability or activity in six subsequent cycles. Leaching of palladium did not take place [216].

Palladium finds application in hydrogen-based reaction because of its unique capability of absorbing hydrogen even at mild conditions. The activation energy needed for dissociative adsorption of hydrogen on its surface is significantly lesser [217].

Palladium catalysts are widely used in the pharmaceutical and agrochemical industry. Prosulfuron is a broadspectrum herbicide synthesized by Matsuda-Heck reaction of 2 sulfonatobenzenediazonium on 1,1,1-trifluoropropene, using palladium as a catalyst.

Reactions in which the products of the catalyzed coupling reaction require hydrogenation have a particular advantage. The spent homogeneous catalyst is used in the hydrogenation reaction as a heterogeneous catalyst once it is precipitated out. This is easy to separate and is recyclable. Using homogeneous palladium-catalyzed coupling has better selectivity than the Friedel-Craft type reaction, but there are certain drawbacks. Palladium is costly, and it has limited reusability and results in contamination, producing a lot of waste.

Even though they are not environmentally benign, catalytic Friedel-Craft-type reactions are still used in pharmaceutical industries. They are common, and it is easy to identify and rectify any reaction difficulties. Pharmaceutical companies would like to replace Friedel-Craft reactions on inactivated systems because of the large amount of acidic and aluminous waste produced when quenched with water. Product purification generates the most amount of waste is in the pharmaceutical industry. Improved catalyst technologies are required for better selectivity, activity, and turnover numbers (TON's) [218]. A greener approach in the reduction reaction of aminophenols was initiated by Kamal who successfully synthesized an agar biopolymer supported copper oxide nanomaterial catalyst which could be reused for up to five times and showed high catalytic activity [219]. Some of the applications of gold as a catalyst in fine chemical synthesis along with the reactions in which they take part are listed in Table 10.

\subsection{Nanomaterials as Catalyst in Hydrocarbon Processing}

3.3.1. Carbon Nanotubes. Carbon nanotubes (CNTs) are cylinder-shaped molecules made up of rolled-up single-layer carbon atom sheets (graphene). Single-walled nanotubes (SWCNT) have a diameter of less than 1 nanometer $(\mathrm{nm})$, while multiwalled nanotubes (MWCNT) have diameters of more than $100 \mathrm{~nm}$ and are made up of multiple concentrically interconnected nanotubes. Their length might range from a few micrometers to millimeters. CNTs are chemically connected with $\mathrm{sp}^{2}$ bonds, an incredibly strong form of molecular contact, just like its building block graphene. This property, combined with carbon nanotubes' natural tendency to rope together due to van der Waals forces, allows for the development of ultrahigh strength, low-weight materials with highly conductive electrical and thermal properties. This makes them very appealing for a wide range of applications [30].

3.3.2. Nano-SAPO-3. Nano-SAPO-3 catalysts have also been synthesized and then modified with cerium and zirconium using wet impregnation methods. They were then experimented with thermal cracking and thermal catalytic cracking of naphtha. It was found that they had better performance for the catalytic cracking [30].

\subsubsection{Mechanochemically Produced Nanocatalysts.} Mechanochemically prepared $\mathrm{Ag} / \mathrm{Al}_{2} \mathrm{O}_{3}$ nanomaterials have been prepared and tested in the selective catalytic reduction of NOs using hydrocarbons in the presence and absence of hydrogen. A significant increase in the activity especially at lower temperatures where noted compared to traditional catalysts. This was found due to the surface modifications which provided an increased affinity towards hydrocarbons relative to water. This reduced the activation barrier for the reduction of $\mathrm{NO}_{x}$. Transitional metal nanoparticles (NPs) such as $\mathrm{Co}, \mathrm{Fe}$, and $\mathrm{Ni}$ can also be produced by extending dry ball milling methods. These are then used as conventional catalysts in the hydrogenation of $\mathrm{CO}$ to hydrocarbons (Fischer-Tropsch process). Bio-oil (containing simple aromatics) production seemed to be enhanced when depolymerised lignin was catalysed using 10\% wt Ni-base aluminosilicate [229].

3.3.4. Core-Shell Nanomaterials. Core-shell nanomaterials have been found out to be effective catalysts in the reduction of hydrocarbons. A nanozyme (core-shell) catalyst composed of an $\mathrm{Ag}$ core and porus $\mathrm{Cu}$ shell was fabricated using a two-step seeded process. The Ag core was grown using $\mathrm{AgNO}_{3}$, and the porous $\mathrm{Cu}$ shell was made by reducing $\mathrm{Cu}(\mathrm{NO})_{3}$ in the presence of hydrazine. The porous shell of $\mathrm{Cu}$ is rich with channels which can be used for cascade reactions. This helps in the $\mathrm{C}-\mathrm{C}$ formation and preventing the diffusion of CO [230]. 
TABLE 10: Synthesis methods using gold as catalysts.

\begin{tabular}{lccr}
\hline Sr. no & Reaction & Application in fine chemical synthesis & Reference \\
\hline 1 & Direct esterification & Methyl ester synthesis & {$[121]$} \\
2 & Oxidative condensation & Amide synthesis & {$[220]$} \\
3 & Hydrogenation-oxidation & Aromatic azo compounds & {$[221]$} \\
4 & Hydrogenation-condensation & Imines & {$[222]$} \\
5 & Hydrogenation-amine-alkyne coupling & Imines & {$[223]$} \\
6 & Oxidation-condensation & Imines & {$[224]$} \\
7 & Hydrogenation-condensation & Substituted secondary amines & {$[222]$} \\
8 & Oxidative condensation & Secondary benzylamines synthesis & {$[224]$} \\
9 & Hydrogenation-condensation & Pyrrolidones & {$[225-228]$} \\
10 & Reduction-N-carbamoylation & Carbamates & {$[205]$} \\
\hline
\end{tabular}

3.3.5. Layered Double Hydroxides (LDH). Layered double hydroxides (LDH) (which are multifunctional nanomaterials) controlled calcination produces mixed oxides. These mixed oxides have catalytic applications in the polymerisation, condensation, synthesis, etc., in hydrocarbon synthesis. Fe/CaxO catalysts made of different $\mathrm{Ca} / \mathrm{Fe}$ molar ratios were produced from $\mathrm{CaxFe}-\mathrm{LDH}$ precursors. $\mathrm{Fe}$ percentage had a significant impact on the composition, particle size $\mathrm{CO}_{2}$ absorption, and catalytic activity. A maximum of $48.3 \%$ wt was obtained as gasification yield, while $37.48 \%$ vol of $\mathrm{H} 2$ was obtained [231].

3.3.6. Gamma- $\mathrm{Al}_{2} \mathrm{O}_{3}$ Doped with $\mathrm{MnO}_{2}$. Gamma- $\mathrm{Al}_{2} \mathrm{O}_{3}$ doped with $\mathrm{MnO}_{2}$ is effective in the adsorption and oxidation of $\mathrm{SO}_{2}$ in the desulfurization of oil and gas. In this field, one of the latest developments is the synthesis of amphiphilic tungsten oxide with oxygen vacancies. These catalysts remove sulphur at reportedly $100 \%$ efficiency and last up to 15 cycles of recycling [133].

\subsubsection{Highly Dispersed $\mathrm{Cr}_{2} \mathrm{O}_{3}-\mathrm{ZrO}_{2}$ Binary Oxide} Nanomaterials. Catalytic conversion of alcohol to hydrocarbons is effectively catalysed using highly-dispersed $\mathrm{Cr}_{2} \mathrm{O}_{3}-\mathrm{ZrO}_{2}$ binary oxide nanomaterials. The catalytic activities of the binary oxides were found to be greater than that of pure oxides [232].

3.3.8. Hydrogenolysis. Hydrogenolysis is the chemical reaction which leads to the cleaving of a carbon-carbon or carbon-heteroatom single bond or lysis by hydrogen. In the petrochemical industry, the formation and cleavage of $\mathrm{C}-\mathrm{C}$ bonds are critical because the conversion of crude oil into hydrocarbons is required. A lot of catalysts have been explored and implemented for this reaction, but a stable nanocatalyst is yet to come. Fibrous nanosilica (KCC-1) supported ruthenium nanoparticles have been reported as stable and sustainable by Fihir et al. 3-Aminopropyltriethoxysilane was used for the postsynthetic modification of the silica fibres. The functionalized fibers were then reacted with $\mathrm{RuCl}_{3}$ and then reduced with hydrogen to form Ru NP-supported KCC-1. This catalyst showed high activity for the hydrogenolysis of propane and ethane at atmospheric pressure and temperature. Its hexagonal- shaped particles with many corners and sharp edges gave this catalyst superior catalytic activity [233].

3.3.9. Oxidation. Mesoporous nanoparticles (MSNs) like MCM-41 are known catalysts for oxidation. MSNs have been synthesized using microemulsion methods by Yokoi et al. These were $20 \mathrm{~nm}$ on average and $3 \mathrm{~nm}$ in diameter. Epoxidation of different-sized alkenes, cyclohexane, cis-stilbene, and others were carried out using this catalyst. Ti-MSN showed superiority in the experiment with a pretty high conversion percentage. Epoxidation of styrene by immobilizing salicylaldimine transition-metal (copper, manganese, and cobalt) complexes on MSNs with diameters of $120-150 \mathrm{~nm}$ was carried out by Tang and coworkers. Excellent activity of the catalyst was noted. Polyoxometalates (POMs) are also said to be efficient in catalysing oxidation reactions. Epoxidation of geraniol with the benign oxidant $\mathrm{H}_{2} \mathrm{O}_{2}$ was carried out by Sousa and coworkers using polyoxotungstate@ $\mathrm{SiO}_{2}$ heterogeneous nanocatalyst. The alkaline hydrolysis of tetraethoxysilane was used to make $\mathrm{POM} / \mathrm{SiO}_{2}$ nanocomposites utilizing a reverse micelle and sol-gel process [233].

3.3.10. Cumene Conversion. Cumene conversion, or cumene catalytic cracking, which produces benzene and propene, is a commonly used test to determine a catalyst's activity. Using the sol-gel process, Sazegar and coworkers created a mesostructured silica-NP-based solid acid catalyst with 1,2propanediol as the cosolvent, followed by aluminium grafting and protonation (HAlMSN). The catalyst's activity for cumene conversion was next investigated in a pulsed microcatalytic reactor at $227-573 \mathrm{~K}$. Cumene conversion on HAlMSN was shown to be very active, yielding propylene, benzene, and toluene via cracking at protonic acid sites. Furthermore, dehydrogenation at the Lewis acidic sites produced methylstyrene as the primary result at high reaction temperatures. The catalyst's excellent stability and activity allowed for the generation of methylstyrene via a subsequent dehydrogenation step [233].

3.3.11. Olefin Hydroformylation. Hydroformylation is a crucial synthetic reaction with widespread industrial use. Olefins are changed to aldehydes, which can then be further altered into various functional groups including acids, 
amines, and alcohols. In most cases, greater olefin hydroformylation takes place in a single organic phase, and the homogeneous catalyst is frequently damaged during the distillation process. The group of $\mathrm{Li}$ was able to separate the pricey rhodium catalyst by immobilizing the $\mathrm{HRh}(\mathrm{CO})\left(\mathrm{P}\left(\mathrm{m}-\mathrm{C}_{6} \mathrm{H}_{4} \mathrm{SO}_{3} \mathrm{Na}\right)_{3}\right.$ complex onto nonporous fumed silica nanoparticles. In the hydroformylation of 1hexene, this solid catalyst performed quite well. Furthermore, in the presence of basic alkali metal salts such as $\mathrm{Na}_{2} \mathrm{CO}_{3}, \mathrm{~K}_{2} \mathrm{CO}_{3}$, and $\mathrm{NaH}_{2} \mathrm{PO}_{4}$, the catalyst's activity was improved [233].

3.3.12. Esterification Reaction. The use of solid-supported catalysts in the oxidative esterification of alcohols has paved the way for the efficient, long-term, and environmentally friendly production of esters from widely available materials. Aluminium-derivatized monodisperse silica nanospheres (Al-MSS-22) are an intriguing example of a solid-supported catalyst that has been used to successfully synthesize esters. The preparation of the Al-MSS-22 catalyst was achieved by Nyalosaso et al. through a one-step synthesis-functionalization methodology. In esterification reactions of ethanoic acid with alcohols or polyols such as butanol, isoamyl alcohol, ethylene glycol, or glycerol, the catalytic behaviour of Al-MSS-22 in the liquid phase was examined [233].

3.3.13. In Situ Catalytic Upgrading. Yusuf et al. reported that NiMo-oleate promoted the catalytic aquathermolysis of Omani heavy oil. The catalyst reduced viscosity by 69 percent at its maximum $\left(1490-490 \mathrm{cP}\right.$ at $\left.70^{\circ} \mathrm{C}\right)$. The optimum reaction conditions were established in a Parr reactor under nitrogen atmosphere at $277^{\circ} \mathrm{C}$ with catalyst dose, glycerol concentration, and water content of $1 \%, 5 \%$, and $10 \%$, respectively [234].

3.3.14. Catalytic Cracking. In both the laboratory and the field, Chao et al. used a bifunctional catalyst, alkyl ester sulfonate copper, for the aquathermolysis reaction of heavy oil. The viscosity of heavy oil fell by 90.72 percent with a 10.12 percent conversion of heavy to light content employing $0.3 \mathrm{wt}$ \% catalyst, according to laboratory results. The catalyst's exceptional activity was further validated by the field test findings [234].

3.3.15. Hydroprocessing of Crude Oil. Palos et al. [137] produced NiMoP-based catalysts on several supports (MCM-41, SBA-15, and $\mathrm{HY}-\mathrm{Al}_{2} \mathrm{O}_{3}$ ). In the hydroprocessing of light cycle oil, their catalytic activity was examined (LCO). The goal of the research was to increase sulfur removal while lowering the polyaromatics content of LCO. At $320-400^{\circ} \mathrm{C}$, the hydroprocessing reaction was carried out in a fixed bed reactor under a trickle bed regime. Because of the hierarchical pore architecture, which allowed higher LCO accessibility, $\mathrm{NiMoP} / \mathrm{HYAl}_{2} \mathrm{O}_{3}$ had the best hydrodesulfurization activity of the three catalysts [234].
3.3.16. $\mathrm{TiO}_{2}$ Nanoparticles. $\mathrm{TiO}_{2}$ nanoparticles (NPs), nanofibres (NFs), and nanotubes (NTs) were synthesized and tested for hydrocarbon oxidation by Qadir et al. They later carried out the allylic oxidation of 1,5-cycloctadiene, indane, and cyclohexane [235].

\subsection{Nanomaterials as Catalyst in Renewable Energy}

3.4.1. Catalytic Transformations of Renewable Substrates. For renewable chemical plants to achieve efficient catalytic conversion of $\mathrm{CO}_{2}$, a high pressure concentrated stream of $\mathrm{CO}_{2}$ is currently needed. There are two approaches used in designing renewable substrates, (Figure 14). In a bottom-up path, the core, which is usually inorganic, is covered with either an inorganic or organic shell before being used as a core-shell catalyst. This is most commonly seen in biomass and $\mathrm{CO}_{2}$ transformations. The shell covers and stabilises the inner catalytic center, allowing reactions with forcing conditions to proceed more quickly. Core-shell structured materials are known to be used for gas separation and, in this case, to store $\mathrm{CO}_{2}$. This material has a sorption capacity which has $30 \%$ more $\mathrm{CO}_{2}$ than the actual shell which progresses to selective $\mathrm{CO}_{2}$ capture. The reduction of $\mathrm{CO}_{2}$ can be modified by using an $\mathrm{Au} / \mathrm{Pd}$ core-shell catalyst and by modifying the thickness of Pd shell coating the Au core.

Top-down techniques have also been used, in which the NM surface is inscribed so that the core structure feels different when compared to the shell. The interactivity which takes place between the 2 components strengthens the catalytic processes in both bottom-up and top-down generated core-shell catalysts as shown in the figure, possibly through the modifications in the electronic nature by the noncatalytic component, or by each component playing a distinct role in the catalytic cascade reactions [236].

3.4.2. Remediation of Wastewater. In recent work, it was found that the trivial graphite layer which was extracted from pencils were used as anode after certain modifications with $\mathrm{TiO}_{2}$ nanoparticles to treat and reform nodularin (empirical formula: $\mathrm{C}_{41} \mathrm{H}_{60} \mathrm{~N}_{8} \mathrm{O}_{10}$ ) which is a cyanotoxin present in water bodies which induces oxidative stress and promotes tumour in animals.

This procedure helps control the processes to its fullest extent for enabling complete mineralisation. Moreover, this method is utilized to treat a broad spectrum of wastewaters containing different types of contaminants at different quantities. It is one of the most productive wastewater restorative procedures due to its cost-effectiveness, simplicity, compactness, and robustness, as well as the potential for scale-up applications. A flowchart is given to explain the abovementioned procedure, (Figure 15) [42].

Environmental sensors are a novel approach to feel and understand the environment, having an enormous capability in environmental sciences. The risk generated by the outbreak of water pollution necessitates the quick progress of systems for monitoring water quality. Given their distinctive localised surface plasmon resonances, plasmonic NMs, for example, are intriguing prospects for the construction of biosensors 
BOTTOM UP APPROACH

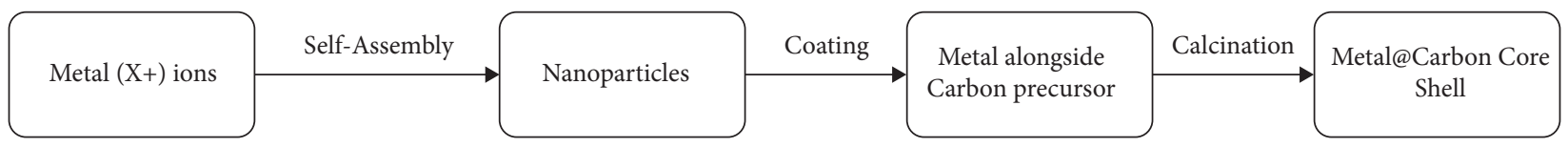

TOP DOWN APPROACH

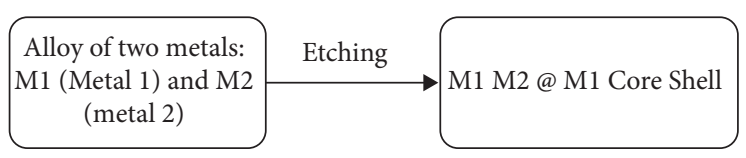

FIGURE 14: Two different approaches for designing renewable substrates.

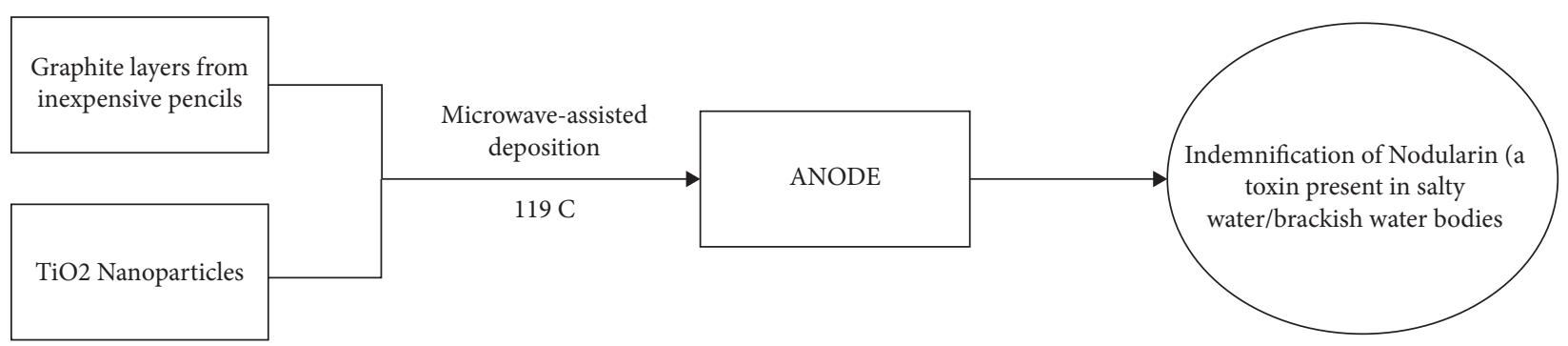

FIGURE 15: Remediation of nodularin using microwave-assisted deposition-based anode.

which may be sensitive. Pollution remediation should receive more awareness and consciousness based on environmental studies performed by environmental sensors [237].

Metallic nanoparticles have come out to be of great interest given the fact that they have copious applications in degrading different kinds of VOCs present in wastewater such as pesticides, impurities, and toxic organic compounds.

When it comes to degrading PCCPs, semiconductor nanocatalysts are particularly effective since they require low operating temperatures and are highly effective even when low concentrations are present. The photoexcitation of electrons in the catalyst is the basic operating mechanism of photocatalysis.

Conduction Band: light irradiation produces holes and expelled electrons.

The holes $\left(\mathrm{h}^{+}\right)$are captured by $\mathrm{H}_{2} \mathrm{O}$ molecules and produce $\mathrm{OH}$ radicals which when reacted with the wastewater containing organic compounds oxidise them, thereby converting them to water and gaseous breakdown products.

$\mathrm{TiO}_{2}$ and $\mathrm{ZnO}$ have been studied extensively to be found as two of the most widely used photocatalysts for wastewater treatment due to their wide band gap as well as their high reactivity. Moreover, their properties have also been studied properly including the factors which will influence their catalytic power such as dose, $\mathrm{pH}$, and band gap energy [238]. $\mathrm{CdS}$ nanoparticles have sparked a lot of attention as a photocatalyst for wastewater treatment of industrial dyes [239]. However, metallic nanocatalysts such as $\mathrm{TiO}_{2}$ and $\mathrm{ZnO}$ are efficient only at ultraviolet radiations which lead the researchers to think about mechanisms which can segue into visible region which is much feasible. To achieve this feat, it was proposed to use certain mechanisms such as hybridize nanoparticles with semiconductors, doping metal impurities, and so on. In solar spectrum, both $\mathrm{TiO}_{2}$ and $\mathrm{ZnO}$ albeit them having a wide gap which do not absorb a lot of portions, thereby decreasing the efficiency. But loading their surface with metals decreases the band gap energy leading to the metal oxides absorbing major portion.

Many dopants are employed to improve and maximise the surface area of metal oxide nanostructures, including Co, $\mathrm{Si}, \mathrm{Mn}$, and Cr. Nitrogen is also thought to be the most viable and cost-effective dopant for industrial use.

Doping nanomaterials has two major advantages [240]:

(i) Reduces the catalyst's surface area

(ii) Protects the nanocomposite from size reduction, morphology, and any change per se which might affect their working/efficiency

$\mathrm{CuO} / \mathrm{ZnO}$ can be fabricated as a photocatalyst which aid the breakdown of a red textile wastewater which might pose to be a problem for the aquatic life. This is done under visible light. When compared to unmodified $\mathrm{ZnO}$, they achieved almost twice as much amount of photocatalytic degradation with $\mathrm{CuO} / \mathrm{ZnO}$. Similarly, an organic homopolymer polyaniline (PANI) was modified with $\mathrm{ZnO}$ nanocomposite which allowed the removal of pollutants present in wastewater up to $99 \%$ [238].

Similar to semiconductor-based nanocatalysts used to degrade the organic pollutants, photocatalysts are, on the other hand, used as antimicrobial agents to inactivate pathogenic bacteria. $\mathrm{TiO}_{2}$ could be used as an effective catalyst, but the separation of the immobilized nanoparticles after the reaction is complete is relatively difficult. However, this could always be solved by increasing their effectiveness 
by modifying/doping them with other additives. $\mathrm{Ag}$ nanoparticles modified on the $\mathrm{TiO}_{2}$ are quite effective in terms of the removal of Escherichia coli, an anaerobic bacteria, than the conventional $\mathrm{TiO}_{2}$. This can be associated to the increase in surface area of $\mathrm{TiO}_{2}$ which therefore provides more area and environment for the microorganisms to degrade. Moreover, the presence of extracellular polymeric substance (EPS) is very essential since it allows us to find out the kinetics of these antibacterial reactions. Ergo, to achieve high photocatalysis efficiency for wastewater disinfection, it is critical to eliminate EPS.

Apart from the abovementioned catalysts, Fenton-based catalysts were also found to be very useful for removing toxic organic pollutants from wastewater. As we know, Fenton's reagent is a hydrogen peroxide $\left(\mathrm{H}_{2} \mathrm{O}_{2}\right)$ solution containing ferrous iron sulphate as a catalyst for oxidising pollutants or waste fluids. Organic molecules such as trichloroethylene (TCE) and tetrachloroethylene can be destroyed using Fenton's reagent. Although this reaction is quite useful in terms of its effectiveness of wastewater treatment, it has its disadvantages. For the optimum reaction to take place, a $\mathrm{pH}$ of 3 is needed. Moreover, there is a consistent loss of catalyst material which decreases the economic feasibility of the latter. This can be solved by employing $\mathrm{Ni}, \mathrm{Zn}, \mathrm{Co}$, and $\mathrm{Cu}$ based spinel ferrites because of their unique magnetic and electrical properties. The inclusion of these metals in the morphology changes certain characteristics of ferrites, mainly their stability, thereby improving the catalytic efficiency even more.

Nanomaterial catalysts can be used in the degradation of organic contaminants in wastewater treatment. Noble metal nanoparticles have a high potential for the destruction of a wide range of pollutants. When compared to traditional treatment procedures, nanocatalysts certainly have an edge due to their potential to target resistant molecules, improve the kinetics of the reaction, and convert wastes into valuable byproducts. Although there are many advantages to using nanocatalysts, there are some drawbacks, such as the expensive cost of metal nanoparticles $(\mathrm{Pt})$ and the difficulty of recovering them for reuse. To troubleshoot this, we can try to hybridize these metals with transition metals which are very economically viable, thereby decreasing the cost of the overall water treatment. $\mathrm{Pt} / \mathrm{Ni},\left(\mathrm{Pd} / \mathrm{Fe}_{3} \mathrm{O}_{4}\right)$ catalysts can be manufactured with the capacity to re-extract the catalyst magnetically. They also have a lot of activity when it comes to treating halogenated organic contaminants. $\mathrm{Fe}_{2} \mathrm{O}_{3}$ catalyst, in combination with other chemicals, has a strong effect on phenol oxidation and removal from wastewater. Other ions, such as $\mathrm{Ca}, \mathrm{Mg}, \mathrm{Na}$, and others, were tried and evaluated, but they had no effect on the kinetics of phenol oxidation and removal [239].

3.4.3. $\mathrm{CO}_{2}$ Fixation. For $\mathrm{CO}_{2}$ capture under the required pressure conditions, nanomaterials such as mesoporous silica functionalized by amine, COFs, porous organic polymers (POPs), MOFs, polymers made from organic compounds, porous carbon-based structures, and porous inorganic oxides of metals with a simple morphological framework have been studied extensively.
Given the wide range of reactions that are used during $\mathrm{CO}_{2}$ fixation, as well as the presence of a metal that can catalyse the reactions at certain places, $\mathrm{CO}_{2}$ fixation reactions over porous NMs will rightly be investigated in the future. $\mathrm{CO}_{2}$ fixation processes attached to high surface area NMs have been studied in the manufacture of fine chemicals, value added chemicals, DMCs $\left(\mathrm{C}_{3} \mathrm{H}_{6} \mathrm{O}_{3}\right)$, which is important in the context of successful $\mathrm{CO}_{2}$ use. Applications of catalysts used in renewable energy have been demonstrated in Table 11.

This makes it a ubiquitous carbon source for two reasons:

(1) Increasing the length of the chain as well as functionalizing it

(2) A source of environmentally beneficial renewable energy

Aside from chemical fixation, the transformation of carbon dioxide into value-added chemicals is conceivable via photocatalytic and electrocatalytic routes. Metallic nanoparticles often play an important part when it comes to accelerating the said reactions. For the electroreduction of $\mathrm{CO}_{2}$ to formate, Pd NPs demonstrated high catalytic efficiencies. On the other hand, Au/Pd NPs that are carried on $\mathrm{TiO}_{2}$ nanotubes can boost the photocatalytic conversion of $\mathrm{CO}_{2}$ to methane [241].

3.4.4. Hydrogen Uptake. Rising petroleum costs, in particular, and global energy demand in general, were caused by greenhouse gas emissions and the depletion of fossil fuel supplies. This, in turn, stimulated the use of renewable energies as well as the development of various methods to produce them from renewable sources. Many experts view the use of pure hydrogen production technologies in this venue to be an alternate way to replacing fossil fuels. This is mainly because $\mathrm{H} 2$ fuels have the minimum emission, thereby leading to protecting the environment unlike conventional fossil fuels. Steam reforming is the most frequently used method for generating hydrogen. This is a wellestablished approach that has been in use since the 1930s. This technique did, in fact, supply almost $60 \%$ of the hydrogen production around the world. The fundamental advantage of this extensive production range is its low cost. However, the enormous amount of $\mathrm{CO}_{2}$ emissions produced by steam reforming is a major drawback. Despite this, hydrogen synthesis from biomass or waste is regarded as one of the most effective and ecologically sound processes [251].

Bogdanovic and Schwickardi's pioneering work established that $\mathrm{TiCl}_{3}$ functions as a catalyst to reduce $\mathrm{NaAlH}_{4}$ to produce hydrogen as well as to adsorb hydrogen. This has given rise to hope that alanates could be useful materials to store hydrogen. When $\mathrm{NaAlH}_{4}$ is layed on a nanomaterial like CNT or $\mathrm{C}_{60}$, the energy which may be required to separate the hydrogen out reduces to quite an extent. This process is roughly identical to when Ti is substituted at the $\mathrm{Na}$ site in $\mathrm{NaAlH}_{4}$. Na contributes an electron to become electronegative which weakens the link between $\mathrm{Al}$ and $\mathrm{H}$, thereby lowering the hydrogen desorption energy and improving the kinetics. The $\mathrm{C}_{60}$ molecules' full surface is most 
TABLE 11: Catalyst used in laboratories, their products, and respective applications.

\begin{tabular}{|c|c|c|c|c|}
\hline $\begin{array}{l}\text { Sr. } \\
\text { no. }\end{array}$ & Catalyst used & Product & Application of product & Reference \\
\hline 1 & $\begin{array}{l}\mathrm{Fe}, \mathrm{Ru}, \text { and Ir homogenous } \\
\text { complexes }\end{array}$ & Formic acid (FA) & $\begin{array}{c}\text { Due to its large volumetric } \mathrm{H}_{2} \text { storage capacity, moderate } \\
\text { toxicity, and flammability in ambient settings, it is an excellent } \\
\text { hydrogen energy carrier }\end{array}$ & {$[241]$} \\
\hline 2 & Ni complexes/boryl ligand & $\mathrm{HCHO}$ & $\begin{array}{l}\text { Used in the fabrication of different resin products. Preservative } \\
\text { in foods and paints. }\end{array}$ & [242] \\
\hline 3 & $\begin{array}{l}\text { Poly(ethyleneimine)- } \\
\text { functionalized Ir complex }\end{array}$ & FA & In livestock feed as a preservative and antibacterial agent & [243] \\
\hline 4 & $\mathrm{Pd} / \mathrm{ZnO} / \mathrm{Al}_{2} \mathrm{O}_{3}$ catalyst & $\mathrm{CH}_{3} \mathrm{OH}$ & Chemical storage carrier for excess $\mathrm{H}_{2}$ generated & {$[244]$} \\
\hline 5 & $\mathrm{Cu}-\mathrm{ZnO}-\mathrm{Al}_{2} \mathrm{O}_{3}$ catalysts & $\mathrm{CH} 3 \mathrm{OH}$ & Used in renewable energy production & [244] \\
\hline 6 & $\mathrm{Cu} \mathrm{NPs} / \mathrm{mesoporous} \mathrm{alumina}$ & DME (dimethyl ether) & Transportation fuel for IC engines & {$[245]$} \\
\hline 7 & $\mathrm{Cu} / \mathrm{ZnO} / \mathrm{Al}_{2} \mathrm{O}_{3}$ hybrid NPs & DME & Replacement of diesel in the future & [245] \\
\hline 8 & $\mathrm{Cu}-\mathrm{ZnO}-\mathrm{ZrO}_{2}$ & DME & As a substitute for other fuel cells in power generation & [246] \\
\hline 9 & Ce and $\mathrm{Mg}$ & $\mathrm{CH}_{4}$ & $\begin{array}{l}\text { Natural gas is made up primarily of methane, which is widely } \\
\text { used as a fuel }\end{array}$ & {$[247]$} \\
\hline 10 & $\mathrm{Ni}$ direct methanation & $\mathrm{CH}_{4}$ & Used as fuel & [247] \\
\hline 11 & $\mathrm{Mg}_{2} \mathrm{NiH}_{4}$ & $\mathrm{CH}_{4}$ & Sometimes used as rocket fuel after combining with oxygen & [247] \\
\hline 12 & $\mathrm{Co} / \mathrm{SiO}_{2}$ & $\mathrm{CH}_{4}$ & Fuel for turbines & [248] \\
\hline 13 & $\mathrm{Au} \mathrm{NPs}$ & Formamide $\left(\mathrm{CH}_{3} \mathrm{NO}\right)$ & Pharmaceutical products & [249] \\
\hline 14 & Pd-supported catalysts & Formamide $\left(\mathrm{CH}_{3} \mathrm{NO}\right)$ & Synthesizing nitrogen-bridged heterocycles & [249] \\
\hline 15 & Hydrosilanes & Formamide $\left(\mathrm{CH}_{3} \mathrm{NO}\right)$ & Production of isocyanides for medicines & [250] \\
\hline 16 & $\mathrm{Ni} / \mathrm{PdCl}_{2}$ catalysts & $\begin{array}{l}\text { Hydrocarboxylated } \\
\text { alkenes }\end{array}$ & To make sure $\mathrm{CO}_{2}$ as a feedstock is utilised to the fullest extent & [247] \\
\hline 17 & Lewis acidic Zn Salts & Wide range of ethers & Fats, oils, waxes, and perfumes & {$[241]$} \\
\hline 18 & Tin-based complex & Dimethyl carbonate & Drug development & [241] \\
\hline 19 & Finely distributed Ag NPs & Cyclic carbonates & Paper industry & {$[241]$} \\
\hline 20 & AgNPs@CO-MOF & Alkyne carboxylates & Organic synthesis catalysts & [241] \\
\hline 21 & Pd NPs & Benzylic alcohol & Solvent for paints, waxes, and coatings & [249] \\
\hline 22 & Ni NPs & Aryl bromides & Medicinal chemistry & [247] \\
\hline
\end{tabular}

likely accessible for bonding with $\mathrm{NaAlH}_{4}$. In case of graphite particles, their particle sides will be available for $\mathrm{NaAlH}_{4}$, but CNTs are well known in case of aggregating and forming molecules that are grouped together, reducing the amount of surface area available to $\mathrm{NaAlH}_{4}$ [252].

A sequence of $\mathrm{Ni}$ nanostructured catalysts was produced on $\mathrm{Al}_{2} \mathrm{O}_{3}, \mathrm{MgO}$, and their mixed supports, and their physiochemical properties were investigated. It was therefore proved that the $\mathrm{Al} / \mathrm{Mg}$ ratio had a substantial impact on nickel catalysts. The catalytic activity of $\mathrm{Ni} / \mathrm{Al}_{2} \mathrm{O}_{3}$ and $\mathrm{Ni} /$ $\mathrm{MgO}$ materials was found to be less than their $\mathrm{Ni} / \mathrm{A}_{x} \mathrm{M}_{y}$ equivalents. The $5 \mathrm{wt} \% \mathrm{Ni} / \mathrm{A}_{2} \mathrm{M}_{1}$ catalyst demonstrated relatively high catalytic activity to obtain glycerol (92\%) as well as decent selectivity for producing hydrogen (76\%). Overall, this new $\mathrm{Ni} / \mathrm{A}_{2} \mathrm{M}_{1}$ catalyst is an innovative material for producing $\mathrm{H}_{2}$ via the aqueous phase reforming (APR) process. The electron affinity of numerous carbon substrates has revealed how the hydrogen sorption mechanism is substantially associated with the electron affinity. The greater the electron affinity demonstrated by the substrate, the more likely it is that $\mathrm{Na}$ will donate its electron to it. The substrate had a significant impact on the hydrogen removal energy. When tested, it was found that $\mathrm{C}_{60}$ fullerene was a superior catalyst than CNTs. Theoretically, this is owing to its extremely high curvature. However though, in the dehydrogenation of $\mathrm{NaAlH}_{4}, \mathrm{CNT}$ may be a better catalyst than $\mathrm{C}_{60}$ fullerene [112].
3.4.5. Water Splitting. Water splitting produces hydrogen, which is an appealing possibility for bridging the gap between the energy demand and supply in the future. The reaction is split into two different parts: hydrogen evolution reaction (HER) and oxygen evolution reaction (OER). Direct use of sunlight (photocatalysis) and energy generated from renewable resources are the two most explored low-cost approaches (i.e., electrocatalysis). Given that the two reactions occur at the cathode and anode separately, it is possible to modify the design of HER and OER catalysts separately in the electrocatalytic method. In the last few decades, many catalysts for both photocatalytic and electrocatalytic methods have been proposed and extensively explored [253].

Development of effective catalysts is a must for the abovementioned reactions to take place at a faster rate compared to conventional rates [253].

For OER and HER to take place effectively, strong electrocatalysts must be exploited and assessed. pH-based catalysts are good, but they are still in their starting stages. However, nanomaterial-based catalysts are quite widely known and accepted as the rulers of electrocatalysts. For $\mathrm{HER}, \mathrm{Pt}$ based catalysts and for $\mathrm{OER}, \mathrm{IrO}_{2}$ can be considered as an effective catalyst. Iridium, an emerging metal which also belongs to the platinum group, has a high conductivity and superb catalytic activity which acts as a boon in terms of acting as a good electrocatalyst. 
In both acidic and alkaline media, the HER properties of Ir are inferior to $\mathrm{Pt}$, which may be linked to Ir's weak dispersion as its enthalpy of atomization is much higher than Pt. The enhanced HER properties of some Ir-support hybrid systems are better than the conventional Ir based catalysts due to their components being well scattered. Ir-based nanomaterials which include Ir metals, alloys, oxides, different facets of oxides, and other compounds have been tested in OER and HER to find out whether they are good in terms of their catalytic activity. An effort has also been taken to redefine, modify, and enhance the morphology of the aforementioned materials to infer if the properties of such materials have any change.

As far as water splitting was concerned, the coupling of HER and OER electrocatalysts was considered, and for this, the development of bifunctional catalysts was critical. Therefore, hybrid systems were generated from two or more nanomaterial-based components to obtain properties of all the individual components. The main advantages of having a bifunctional catalyst are integrating OER and HER components together to form effective composites; allowing redox reactions to take place for fine tuning the catalysts; and improving the intrinsic properties of the said catalysts. However, much research is still pending in this case, and must be done to advance, utilize, and exploit these Ir-based electrocatalysts to their fullest extent to get the product as per the desired application [254].

Yoshida and coworkers [255] developed a new and a novel method for efficient water splitting that enclosed $\mathrm{Rh}$ nanoparticles in a thin porous lanthanum oxide shell to keep the Rh surface accessible for protons while avoiding contact with $\mathrm{O}_{2}$. As a result, $\mathrm{H}_{2}$ formation in water splitting was significantly improved. Similarly, different catalysts in industries also use a porous surface as a protection for the catalysts from poisons.

$\mathrm{Wu}$ et al. made a proposal involving porous hybrid of mixed oxide nanosheet array made up of $\mathrm{Co} / \mathrm{NiO} / \mathrm{NiFe}_{2} \mathrm{O}_{4}$ atop a foam of $\mathrm{Ni}$ which was found to be a stable low water splitting device [256].

Layered perovskites are proving to be one of the most effective materials for hydrogen separation. A perovskite is a crystal structure that is similar to calcium titanate $\left(\mathrm{CaTiO}_{3}\right)$. $\mathrm{ABX}_{3}$ is the chemical formula for perovskites, where the cations are $\mathrm{A}$ and $\mathrm{B} ; \mathrm{X}$, an anion which bonds to both $\mathrm{A}$ and $B$. The layered structure lowers electron-hole recombination and hence plays an important role in controlling the activity of water splitting, along with factors like cation valency and layer thickness. For photocatalytic water splitting [257], Busser et al. investigated the characteristics of $\mathrm{Cr}_{2} \mathrm{O}_{3}$ nanoparticles placed on (111)-layered perovskite $\mathrm{Ba}_{5} \mathrm{Ta}_{4} \mathrm{O}_{15}$.

Metal-organic frameworks are used to make a material that has porous nitrogen-doped carbon materials enclosing cobalt and tungsten carbide to form Co/W-C@NCNSs (MOFs). MOFs at the nanoscale level were created using a microwave technique. In an alkaline medium, it was discovered that Co/WC@NCNSs performed better than OER and HER when utilising a water electrolyser. This was due to the carbon layer's protective properties. As a result, it was determined that cobalt/tungsten carbide-based NCNSs can be used as a superb material to split water via electrocatalytic routes [258].

Emerging 0D carbon quantum dots have also been employed in hydrogen production to improve photocatalytic performance. Carbon quantum dots combined with $\mathrm{MoS}_{2}$ produced through a hydrothermal procedure demonstrated strong reduction activity for hydrogen evolution [259].

2D chalcogenides are semiconductors that may be utilised in photocatalytic reactions for transforming solar energy into chemical fuels. They have quite the potential for solving global energy shortages and pollution. 2D cadmium sulphide (CdS) nanosheets are one of the most commonly used 2D chalcogenides. It has a high electrocatalytic activity, allowing it to be employed in water splitting to generate hydrogen [260].

By using an external mechanical force to produce a piezopotential, piezocatalysis can convert mechanical into chemical energy directly. It has an advantage over other approaches in that it may be employed in low-light circumstances and uses available low-frequency vibration energy sources. By garnering ultrasonic vibrations, we can fabricate piezopotential-driven water splitting to create $\mathrm{H}_{2}$ utilising $\mathrm{ZnO}$ and $\mathrm{BaTiO}_{3}$ micro/nanofibers [261].

3.4.6. Advanced Electrolysis Fuel Cells. ORR (oxygen reduction reactions) are one of the most significant molecular reactions which are used in many industrial applications. Fuel cells are used to generate electricity by this reaction with the support of a cathodic oxygen. To construct such fuel cells, a well-regulated catalyst is required. Platinum catalysts are generally used for this purpose, but it should be realized that platinum has CO poisoning effects and a couple more disadvantages associated with it. Several ORR electrocatalysts in alkaline electrolytes were found to be amusing for the researchers as a low-cost alternative to platinum. But they were still expensive and also far from what the ideal durability for applications looked like. Such catalysts were also found to be fast decaying primarily because of their high catalytic activities.

In polymer electrolytic fuel cell (PEMFC) with acidic electrolyte, ORR ac is increased by using NCNTs prepared via pyrolysis of acetonitrile. The catalytic reactivity metal free carbon-based catalysis is improved by doping with heteroatoms of $\mathrm{N}, \mathrm{O}, \mathrm{P}$ etc., in polymer electrolytic fuel (PEMFC) [112].

Fuel cells and photoelectrocatalytic water splitting etc. can be considered as a next step towards power generation and fuel production processes. With the help of high performance electrocatalysts, the reactivity and reaction kinetics of these processes can be improved. Basic processes used in water splitting like the ORR and OER are hindered due to dormant-like kinetics, while the present valuablenoble metal-based electrocatalysts for the beforementioned mechanisms suffer from extravagant pricing and deficient strength. To overcome these key issues, 2D nanomaterials are utilized. Design principles of catalysts, advanced perspectives in determining where all the active sites and intermediates are present, and different strategies for engineering these materials were studied [262]. 


\section{Results and Discussion}

4.1. Nanocatalysts in Fine Chemical Synthesis. Nanomaterials have enhanced chemical and physical properties as a result of their nanometre level size and large surface area which make nanomaterials good catalysts. A diverse range of nanomaterials are available, and the possibility of its application in fine chemical synthesis is limitless. The constantly increasing demands caused by modernisation puts a heavy pressure on the fine chemical synthesis industry to come up with products. The synthesis of these products must be very efficient with minimum wastage because most of the raw materials available on the planet are nonrenewable.

The synthesis of the catalysts usually used in fine chemical synthesis takes place in strong conditions, consumes a lot of energy, and cannot be derived from renewable feedstocks like nanomaterials can be. Researchers must come up with technologies which are highly efficient but also at the same time are economical. They used to use homogeneous nanomaterials as catalysts before finding out the advantages of heterogeneous catalysts with cleaner reaction profiles along with other added benefits.

Homogeneous catalysts are predominant in the production of fine chemicals because of their good enantioselective property and high turnover frequency. They have a greater molecular interaction with the reactants since they are in the same phase as them, resulting in good catalytic performance. Retrieving these catalysts is difficult and costly techniques like nanofiltration and column chromatography separation are used [184]. Other problems related to homogeneous catalysts include volatile pollutant emissions, need for waste management, and inability to recover catalysts. Using supports by immobilising these catalysts overcomes some of these issues, but this process is costly, their activity reduces with reuse, and (metal) active species are subjected to leaching in solution [13]. Mineral acids are used in the fine chemical industry as acidic catalysts. Environmental concerns tied with using traditional stoichiometric Lewis and Brønsted acid catalysts are forcing an increasing number of fine chemical industries to switch to a greener, sustainable, and environmentally benign method. Heterogeneous solid acid catalysts have been used as a green substitute since they enable easier separation of catalyst and products, reusability, and reduction in waste. The problem with these solid acid catalysts is their strong and corrosive reaction conditions and the waste they produce need to be neutralised. Additional costs need to be spent to purify these waste streams. Hydrotalcite has been used as a solid base replacement for alkaline homogeneous base in organic synthesis in fine chemical synthesis [14]. They can be assisted with ultrasonic or microwave irradiations to promote a benign, polar solvent- free reaction [156, 263]. Energy that would be used for heating for increasing the temperature of the reaction is saved this way, and a lot of time is saved since the reaction time is also shortened. Ultrasonic irradiation of catalysts improves their mass transfer rates and increases the surface area of the catalysts available for the reactants by ultrasonic dispersion. Pillai et al. [264] used ultrasonic assisted hydrotalcite catalysts and reported reduced use of catalysts and faster reactions.

Modifiers, dendrimers, MOFs, and functionalisation with support groups are used to enhance metal nanomaterial properties. Agglomeration of nanomaterials forms nanoclusters which reduce their surface area to volume ratio, and the nanomaterial catalysts do not possess plasmonic behaviour anymore. The catalytic activity reduces as the active sites become blocked. Poisoning of the active sites is another concern leading to reduced catalytic activity. Metal nanomaterials like palladium are subjected to leaching with also reduces the surface area.

Palladium has been extensively used in coupling reactions to produce fine chemicals. Palladium nanoparticles tend to aggregate and after a few consecutive runs and leaching starts to take place, reducing the catalytic surface area. Biffis et al. reported that leaching in palladium was triggered by aryl halide in heck conditions due to the oxidative addition of the halide present on the surface of palladium. Coordinating agents such as sodium acetate has a promoting effect on the metal leaching [265]. Leaching of the catalyst is a major concern associated with using palladium as a catalyst, and a lot of research has gone into coming up with ways to avoid this. The heterogeneous catalysts using supports will start to deactivate due to leaching which leads to degradation of the support. CNT can be used to support palladium since it has a small size which ensures better molecular interaction with the reactants. The surfaces of CNTs can also be functionalised to make them more relevant for the reactions. CNTs have good yields and conversion rates, but their activity reduces after a number of cycles due to leaching and poisoning of the active sites. This limits its sustainability and hence is not a good solution to the problem. Supported liquid-phase catalyst (SLPC) is reusable since it is easy to separate the catalyst from the reaction. The negative interactions between multiple homogeneous organometallic catalysts are avoided when this method is employed since the components are separated by a hydrophobic solvent. This approach could be used in fine chemical synthesis since it significantly reduces the steps involved in the reaction and is a greener option, and leaching does not take place [204]. N-doped MWCNTs as supports for palladium catalysed coupling reactions showed no signs of palladium leaching, and catalytic performance was improved since there was no loss of active sites. There is still a need for a better catalyst which is less expensive with no leaching observed.

Magnetic nanoparticles prove to be good reusable and recyclable catalysts because of the ease of separation with a magnetic field. Their surface can be functionalised and tailored to the needs of the reaction.

Fine chemical synthesis involves multistep reactions which can be reduced using multifunctional catalysts in onepot synthesis. This strategy improves atom economy and simplifies the production of complex fine chemicals which require selective reactions with economical and maximum conversion of reactant atom to product atom.

Synthesis of metal nanomaterials is controllable. Metal nanomaterials and organic nanomaterial polymers have 
large surface areas that can be modified. Organic nanomaterial polymers have easy self-assembly properties. Inorganic nonmetal nanomaterials like carbon nanotube and graphene have high stability and good dispersity. Nanomaterials are also used in drug delivery in the form of nanocarriers to control the drug release into the body and prevent the drug from trans-cis isomerization. They increase the bioavailability of the drug because the level of absorption of the drug by the tissues increases. They protect moieties from chemical and light degradation, thus improving the stability of the drug. There have been attempts to synthesise these nanomaterials from biomass for safer reactions. Nanocarriers can be used to encapsulate and protect nucleic acid which has anticancer properties [266].

4.1.1. Green Chemistry. Green chemistry is an approach to synthesise chemical compounds in a safe and environmentally benign way. It demands optimal use of all the reactants with minimal wastage. Hazardous chemical wastes must be avoided or reduced, and atom economy must be maximised.

It is interesting to note that green chemistry has been found to work in conjunction with nanomaterials. Nanomaterials are being synthesized by environmentally benign and sustainable methods complying with green chemistry, and in return, the use of nanomaterials in the industry leads to a greener, more sustainable route to chemical reactions. Nanomaterials are being used as nanocarriers, catalysts, supports for homogeneous catalysts, in water and air remediation, and biomedical devices [163].

Quantum dots are semiconductor nanoparticles with internal geometry of the bulk solid and molecular electronic properties. They have tuneable properties due to quantum confinement effects [267]. Large number of parameters contribute to the morphology of the synthesised nanomaterial, which makes coming up with alternatives for the current methods tricky. Peng and Peng reported that good quality cadmium selenide, cadmium telluride, and cadmium sulfide quantum dots can be synthesised in a mild one-pot reaction by replacing the toxic and high energy consuming dimethylcadmium solvent precursor used in the traditional method with cadmium oxide [268]. As they continued working on this method, they discovered that use of strong acid anions results in complexes with high stability that will not be able to start the process; hence, weak acid anions are better solvents, and precursors are preferred. They reported that fatty acids, amines, and cadmium acetate can also be used and are better substitutes in synthesising good quality quantum dot [269].

Stabilizers such as cetyltrimethylammonium bromide (CTAB), also a surfactant, and poly(vinyl)alcohol (PVA), a polymer have been demonstrated to have sculpturing effects on the morphology of silver nanoparticles [270]. Gold nanoparticles have bulk metal-like conduction band, and their scattering depends on their morphology and their aggregation. Gold nanoparticles have tuneable properties which increases the possibilities of its application to a wide range [271, 272]. Dahl et al. synthesized ultrasmall gold nanoparticles by taking green route [273]. Metal nanoparticles including gold, nickel, and cobalt among others, have been synthesised using biomass-derived extracts like starch and algae [274-279]. More research should be allocated into defining the chemical nature of the nanoparticle formed. The functional groups attached on the functionalised nanomaterial may be toxic to a living organism, so to make it more biocompatible, polymer has been used for coating. Catalysts are to be used instead of stoichiometric reagents according to green chemistry.

Fine chemical industry employs acid catalysts for the synthesis processes, but mineral acids have been reported to have issues related to its toxic and corrosive nature and additional costs for regenerating them. A greener and more sustainable approach is highly sought after. Heterogeneous solid acid catalysts carry with them the added benefits of heterogeneous catalysis, for example, ease of separation, reusability, and recyclability.

Solid acid catalysts are lesser prone to corrosion, are highly selective, and exhibit good catalytic activity. The problem with solid acids like acidic resins and heteropolyacids is their smaller surface area, even though they can be reused and portray high catalytic activity [280]. Zeolites are microporous inorganic solids having large internal surface areas, good stability selectivity, and tenability properties; however, their micropores are too small and cannot catalyse bulky molecules. Zapata et al. modified the surface of HY zeolites with hydrophobic organic silica to improve its hydrophobic properties [281]. Mesoporous solids like silica have amorphous, irregularly spaced and sized pores which can be altered by intercalation by surfactant species into their layers. Figure 16 demonstrates the formation of mesoporous silica from surfactants. Silica can be obtained from rice husk ash which is a low cost and green resource [282]. ZSM-5, a mesoporous acidic zeolite, can catalyse bulky molecules and have strong acidity [283-286]. Kresge et al. [154] synthesised mesoporous solids with a regularly spaced pores using surfactants. SBA-15 and MCM41 are ordered mesoporous silicas having large and regularly spaced pores which can be immobilised with lewis acids or sulfonic acids to integrate acidic sites [287, 288]. Strong acid sites are incorporated on the walls of SBA-15 when arenesulfonic groups are immobilised on it, making it a desirable catalyst in fine chemical synthesis [287].

The presence of acid sites and supports on solid acid catalysts make them hydrophilic. Sulfonic groups, which are used to make the active sites on solid catalysts more acidic, reduce the hydrophobicity of carbon-based organic frameworks [289-292]. The acid sites are deactivated and the framework can be subjected to hydrolysis in the presence of water. Thus, hydrophobic solid acids are preferred to maintain stability and reusability $[154,280]$. Controlling the hydrophobicity and tuning the acid sites on surface of arene sulfonic modified SBA-15 can increase the scope of this catalyst in fine chemical synthesis. Melero et al. [287] used arene sulfonic-modified SBA-15 for a greener route for Friedel-Crafts acylation instead of the traditional aluminium chloride which have degrading effects on the environment. Friedel-Crafts acylation is used for synthesising 


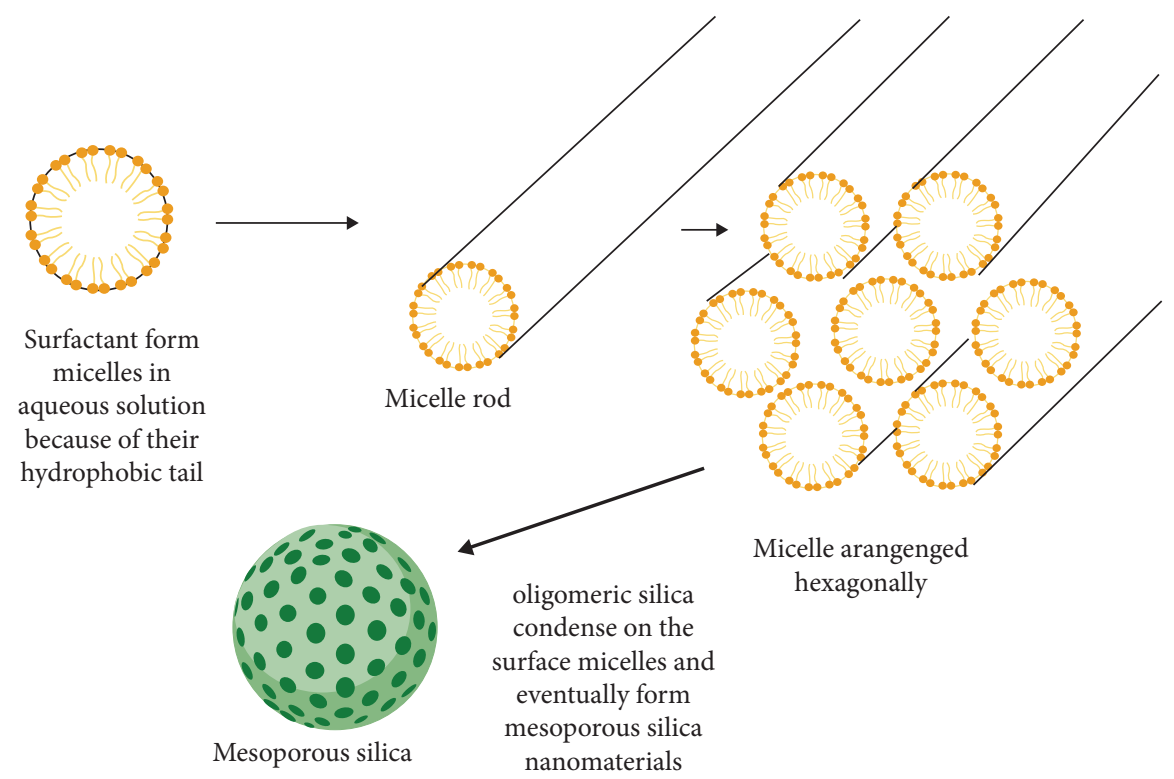

FigURE 16: Formation of mesoporous silica nanoparticle.

aromatic ketone synthesis which are intermediates in fine chemical synthesis.

Polymer-silica nanocomposites have good tolerance against water and are compatible with organic compounds. Incorporating mesoporous materials in polymer-silica nanocomposites ensures even distribution of acid sites and changes the surface wettability. Wang et al. functionalised ordered mesoporous phenolic resin-silica nanocomposites with sulfonic group [293]. This nanocomposite portrayed increased hydrophobic properties, huge BET surface areas, and the acid sites could be controlled.

Wang et al. synthesised 5-hydroxymehylfurural (HMF), a fine chemical, from cellulose in a one-pot process [294]. They used porous polydivinylbenzene (PDVB) solid bases which are hydrophilic and have good wettability for glucose and hydrophobic porous polydivinylbenzene solid acids to catalyse the reaction leading to HMF production [154].

Furfural is usually used for the synthesis of FFA, which has applications in fine chemical synthesis [295-301]. A greener route of FFA synthesis can be through HMF decarbonylation. Jeong et al. came up with a very efficient capillary microreactor for the HMF decarbonylation using $\mathrm{Pd} / \mathrm{Fe}_{3} \mathrm{O}_{4} / \mathrm{SiO}_{2}$ catalyst [302].

Common ways to synthesise lactones are through dehydrogenation or even oxidation of the corresponding $\alpha$, $\omega$-diols and cyclic ketones, but the approaches employed presently for lactone synthesis cause loss of some of the reactants and require strong reaction conditions. Using a catalytic route for oxidation of diols is a greener alternative. Using gold-based catalysts like $\mathrm{Au} / \mathrm{TiO}_{2}, \mathrm{Au} / \mathrm{FeOx}$, gold supported on hydrotalcite (Au/HT) [303], $\mathrm{Au} / \mathrm{MgO}, \mathrm{Au} /$ $\mathrm{Al}_{2} \mathrm{O}_{3}$, and $\mathrm{Au} / \mathrm{SiO}_{2}$ have been proven to give better results than copper-based catalyst which resulted in lower catalytic activity and require more heat. The calcination temperature and the amount of gold used for the catalyst is very important for better activity. Among these, Au/HT proved to be the best catalyst with higher activity and yield, which was accredited due to the basicity of the hydrotalcite support. Hydrotalcite supported gold showed the best activity, stability, and reusability for this reaction when compared with hydrotalcite supported on any other metal like palladium or silver. Using hydrotalcite catalyst assisted ultrasonic or microwave processes provide environmentally benign processes with easier catalyst recovery, safer and easier operation modes along with reduction in waste. They do not need a polar solvent, and the reaction can be conducted at higher temperatures or in vapour phase under heterogeneous condition. Green alternatives to catalysts used in fine chemical synthesis have been demonstrated in (Table 12).

\subsubsection{Green Solvents}

(1) Asymmetric Catalysis under Solvent-Free Conditions. Asymmetric catalysis in benign, solvent-free, and highly concentrated reactions has been used in fine chemical industries for highly stereoselective, cost efficient reactions, better than organic solvent reactions [308]. Generally, a transition metal chiral ligand complex is used as a catalyst in asymmetric catalysis in fine chemical synthesis to direct the reaction into dominating the production of one commercially important stereoisomer product. A greener approach to this catalysis conversion reaction is achieved by using nanomaterials in the chiral catalyst. Functionalization of nanomaterials endorses controllable synthesis and reaction. These metal nanomaterials based chiral catalysts are more accessible owing to their small size, and they are more reactive because of their large surface area and abundance of active sites. Sayed et al. [162] are working on improving their previous approach of using chiral hybrid nanomaterial with metal-organic framework in nitroaldol condensation reaction since the pores did not accommodate the asymmetric 
TABLE 12: Catalysts and their respective application in fine chemical synthesis.

\begin{tabular}{|c|c|c|c|}
\hline $\begin{array}{l}\text { Sr. } \\
\text { no. }\end{array}$ & Catalyst & Application in fine chemical synthesis & Reference \\
\hline 1 & HBeta zeolites & 2-Methylanthraquinone synthesis & [304] \\
\hline 2 & Nafion NR50 & 1,3 -Oxadiazoles derivatives synthesis & [187] \\
\hline 3 & $\begin{array}{l}\text { Titanium silicate (TS)-1 } \\
\text { zeolite }\end{array}$ & Caprolactam (CPL) production & [305] \\
\hline 4 & zeolites & $\begin{array}{l}\text { Zeolite H-BEA is used as a green method for acetylation of substrates such as anisole } \\
\text { (1) Eco-friendly synthesis of jasminaldehyde }\end{array}$ & [306] \\
\hline 5 & Hydrotalcite & $\begin{array}{l}\text { (2) A green alternative base catalysts in Henry reaction, Michael addition, and } \\
\text { Meerwein-Ponndorff-Verley hydrogenation of aldehydes and ketones reactions }\end{array}$ & [14] \\
\hline 6 & $\mathrm{Au} / \mathrm{HT}$ & Lactone synthesis & [303] \\
\hline 7 & $\begin{array}{l}\text { Gold and palladium- } \\
\text { based catalysts }\end{array}$ & Benzimidazoles synthesis & {$[307]$} \\
\hline
\end{tabular}

Henry condensation. Asymmetric catalysis in solvent free conditions has certain drawbacks as follows:

(1) Solvent significantly improves enantioselectivity and catalytic efficiently

(2) Absence of solvent may end up in reactions with high rapid heat generation

(3) Enantioselectivity changes when catalytic concentration changes, and it is difficult to predict the effect on the reactants when the catalyst used is altered

Ionic liquids can be used in place of organic solvents as a green solvent. It can be used in reactions like heck and epoxidation reactions as a stable solvent whose properties are tuneable. Water can be used as a solvent for Suzuki-Miyaura and Sonogashira coupling reactions. Microwave-assisted reactions can improve the solubility of the reagents in water.

Observing the current trends, one can notice that the use of metal and metal oxide nanomaterials is significantly higher than the other types of nanomaterials. Hybrid nanomaterials, CNTs, and fullerenes have a lot of scope, and more research should be conducted using these materials to come up with greener alternatives to the wasteful methods employed in fine chemical synthesis. More research should be allocated into one-pot reactions using heterogeneous catalysts since using homogeneous catalysts has problems relating to catalyst retrieval. It is important to come up with techniques to tackle the problems arising due to difference of coordination chemistry between a mixture of catalysts used in one-pot reactions to increase its application in fine chemical synthesis. Currently, only one catalyst is being used to catalyse multiple reaction which limits its applications.

4.2. Nanocatalysts in Renewable Energy. Catalysis research has changed rapidly during the course of a couple decades to address this growing character as a fundamental constituent of sustainable chemical research. In the past, research on this had been based on a trial-and-error sort of an approach, but new modes of comprehending catalysis have become more available and hence easier to explore. The growing concern for natural resources depletion, carbon dioxide emissions, and other greenhouse gases, which lead to environmental depletion, has driven the transition to a sustainable society to be stepped up mainly because chemistry covers all social goals, from the accessibility of better materials which may decrease the exploitation of resources to innovative solutions that enhance health quality.

In case of energy-based applications, there has been a lot of research which has taken place and is still going strong. Specifically, there have been 3 prime parameters to consider when researching about advanced energy catalysis science:

(1) Advanced feedstocks (fossil energy) transformation catalysts

(2) Advanced catalysts for biological feedstock conversion and especially catalyst conversion to important fuels

(3) Photo- and electropowered carbon dioxide and water conversion advanced catalysts

Catalytic transformation of solar or electrical energy of the carbon dioxide into liquid fuels is a tremendous possibility for new energy sources. In addition, water catalyst production of hydrogen may offer the fuel and process fossil and biomass food supplies with a carbon-free source of hydrogen. These reactions continue to provide great problems for sustainable energy; however, in this field, there are numerous catalytic possibilities to really make a breakthrough. Another significant field, which requires an increased research effort, is catalysis for fuel cells. New innovations are to be made in conjunction with enhancement of the fuel cell components.

Catalytical research in the field of new photocatalysts and electrocatalysts in particular provides state-of-the-art opportunities for solving social issues and making catalytic research even more central in future technologies for a sustainable society [309].

In this paper, we shall be mainly talking about how catalysis is relevant in renewable energy by mentioning things of importance with respect to the following:

(i) Reduction of $\mathrm{CO}_{2}$ emissions and converting them to items which could be used in the future

(ii) Water splitting

(iii) Environmental treatment

(iv) Remediation of wastewater 
(v) Hydrogen uptake

(vi) Fuel cells

Research of these new catalytic domains in regard of the conventional disciplines offers up new prospects for catalysis itself.

4.2.1. Reduction of $\mathrm{CO}_{2}$. There are different ways in which the $\mathrm{CO}_{2}$ reduction takes place. Firstly, talking about reduction via catalysts which do not use Nanomaterials as a medium to enhance their catalytic activity:

(1) $\mathrm{CO}_{2}$ to Methanol. The process of producing methanol from syngas has been in use since quite a while now. The production of methanol from $\mathrm{CO}_{2}$ or as a matter of fact from any given source is counted in methanol economy. The typical technique is to catalyze the RWGS reaction via a 2-catalyst system such as copper and its oxides such as $\mathrm{CuO} / \mathrm{Cu}_{2} \mathrm{O}$. The $\mathrm{CO}$ reduction is followed by a methanol production. The greater ratio $\mathrm{CO} / \mathrm{CO}_{2}$ leads to higher production. Further decreases in $\mathrm{CO}_{2}$ reduce the rate as well, demonstrating a complicated profile, because carbon dioxide can also be fabricated by adding steam to the flux arriving from syngas. However, $\mathrm{H}_{2} \mathrm{O}$ may forbid the reaction.

In the CAMERE process (carbon dioxide hydrogenation to form methanol via a reverse-water-gas-shift reaction) (Figure 17) a RWGS reaction leads to $\mathrm{CO}_{2}$ hydrogenation to methanol (Figure 18). When the water inhibition was removed along with the change of equilibrium, it was proven that the 2-stage mechanism (with the inhibitor $\left(\mathrm{H}_{2} \mathrm{O}\right)$ removal) permits thrice the productivity compared to the initially given 1-stage method.

Samimi et al. modelled and optimized $\mathrm{CO}_{2}$ hydrogenation to $\mathrm{CH}_{3} \mathrm{OH}$ on an industrial scale using the reverse water gas shift reaction (CAMERE). Syngas is first produced in this process by $\mathrm{CO}_{2}$ hydrogenation via reverse water gas shift (RWGS) reaction over a nickel catalyst, and then the syngas is fed into a reactor as the feedstock to produce methanol. The reactor's inner tubes were coated with a water perm-selective membrane to remove $\mathrm{H}_{2} \mathrm{O}$, which was the cause of catalyst poisoning. Furthermore, the results of the CAMERE process's methanol production reactor were compared to the conventional route for producing methanol from coal. In the case of methanol synthesis membrane reactor, the CAMERE process outperformed the CR by producing $20.8 \%$ more methanol.

It was also recently shown by Schlögl et al. that the presence of the interface between copper and zinc oxide incorporates a lattice strain in the copper particles. During the water gas shift reaction, there could be a possibility that the activity of methanol synthesis is more than usual. As a result, fault defects and strain defects could appear in the lattice of copper.

(2) LTMS (Low Temperature Methanol Synthesis). LTMS uses certain homogenous catalysts, which are quite good at hydrogenating materials involving carbonates and formates effectively, under moderate circumstances, and therefore offer novel pathways for synthesizing $\mathrm{CH}_{3} \mathrm{OH}$ from $\mathrm{CO}_{2}$ and
CO (Figure 19). However, in practical terms, the efficient productivity of the catalyst per unit reactor volume is still far enough from that obtainable by employing the standard gasphase methanol catalyst. The method of methanol synthesis from $\mathrm{CO}$ or $\mathrm{CO}_{2}$ is also more cost-effective in terms of the recovery of catalysts and product segregation [310].

(3) $\mathrm{CO}_{2}$ to Short Chain Olefins. Albeit there exist several processes for converting $\mathrm{CO}_{2}$ to short chain olefins, and the essential way of fabricating short chain olefins is steam cracking of natural gas fractions. Olefins of 2 or 3 carbons are ideal for storage and integration of solar energy in the value chain for chemical synthesis, thanks to their high energy formation.

Light olefins, one of the most important building blocks in the chemical industry, can be produced from syngas using Fischer-Tropsch synthesis (FTS). High-temperature FT synthesis would result in light olefins. It is critical to comprehend the effects of metals and promoters and supports as the most influential parameters on the catalytic performance of catalysts. The main active metals in FT catalysts, Fe and Co, are studied in terms of pore size, crystal size, and crystal phase to achieve desirable light olefin selectivity.

The RWGS method can be used here as well, but the only constraint being to maintain the thermodynamic equilibrium of the reaction. Thus, RWGS is rarely avoided and to replace the same, and modified Fischer-Tropsch synthesis reactions can be brought in which has the reactant as syngas $\left(\mathrm{CO}+\mathrm{H}_{2}\right)$. Fischer-Tropsch and modified Fischer-Tropsch synthesis have been demonstrated in Figure 20.

The existence of a bifunctional catalyst that works on integrating RWGS with the FT process would be needed to fabricate short-chain olefins from $\mathrm{CO}_{2}+\mathrm{H}_{2}$ and not syngas. Iron-based FT catalysts that are stacked above alumina can perhaps utilized as a catalyst to provide us with reasonable selectivity, leading to additional study for carrying out major improvements in this field.

Two methanol molecules might dehydrate into $\mathrm{CH}_{3} \mathrm{OCH}_{3}$, which can also be transformed into short chains of olefins. This can be done in the presence of an acid catalyst. Phosphoric acid, zeolites, etc., are the acid catalysts commonly used [310]. The introduction of rhodium catalyst complexes inside the zeolite results in the selective synthesis of lower olefins, especially ethylene. Ethanol is formed by DME isomerization or methanol homologation. This ethanol is catalyzed by Rh post which it gets dehydrated into ethylene. This is an interesting method to steer clear of the selectivity concerns which were described in the conventional FT mechanism.

(4) $\mathrm{CO}_{2}$ to Methane. The Sabatier reaction, which is the hydrogenation of $\mathrm{CO}_{2}$ to $\mathrm{CH}_{4}$, is very popular and has been known since quite a while but has only lately garnered attention. This process necessitates the use of four hydrogen atoms for every one carbon dioxide molecule:

$$
4 \mathrm{H}_{2}+\mathrm{CO}_{2} \longrightarrow 2 \mathrm{H}_{2} \mathrm{O}+\mathrm{CH}_{4}+\text { heat. }
$$

The Sabatier process was first used to take out carbon oxides and their traces from input gas for $\mathrm{NH}_{3}$ production. $\mathrm{CO}_{2}$ methanation has recently sparked considerable 


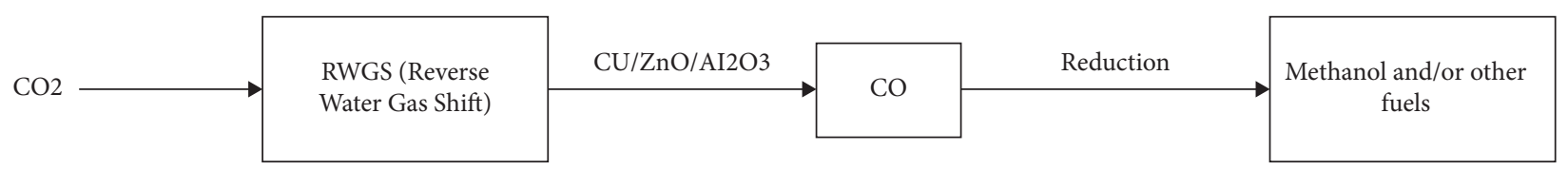

FIGURE 17: Flowchart of RWGS to produce methanol from $\mathrm{CO}_{2}$.

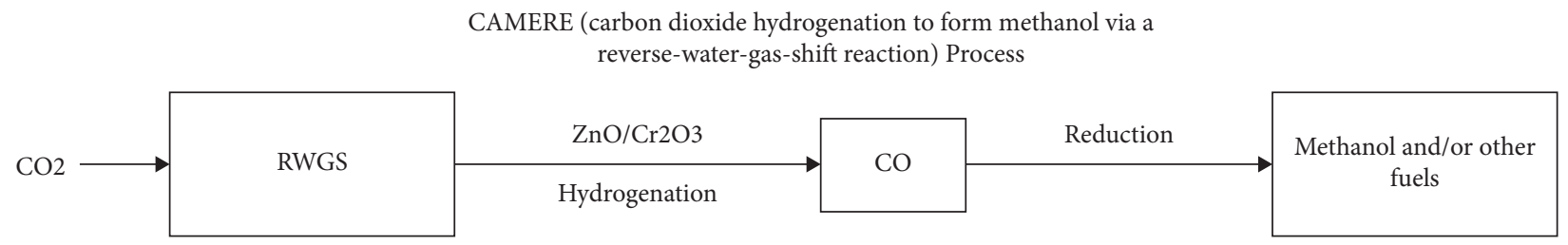

FIGURE 18: CAMERE process for producing methanol.

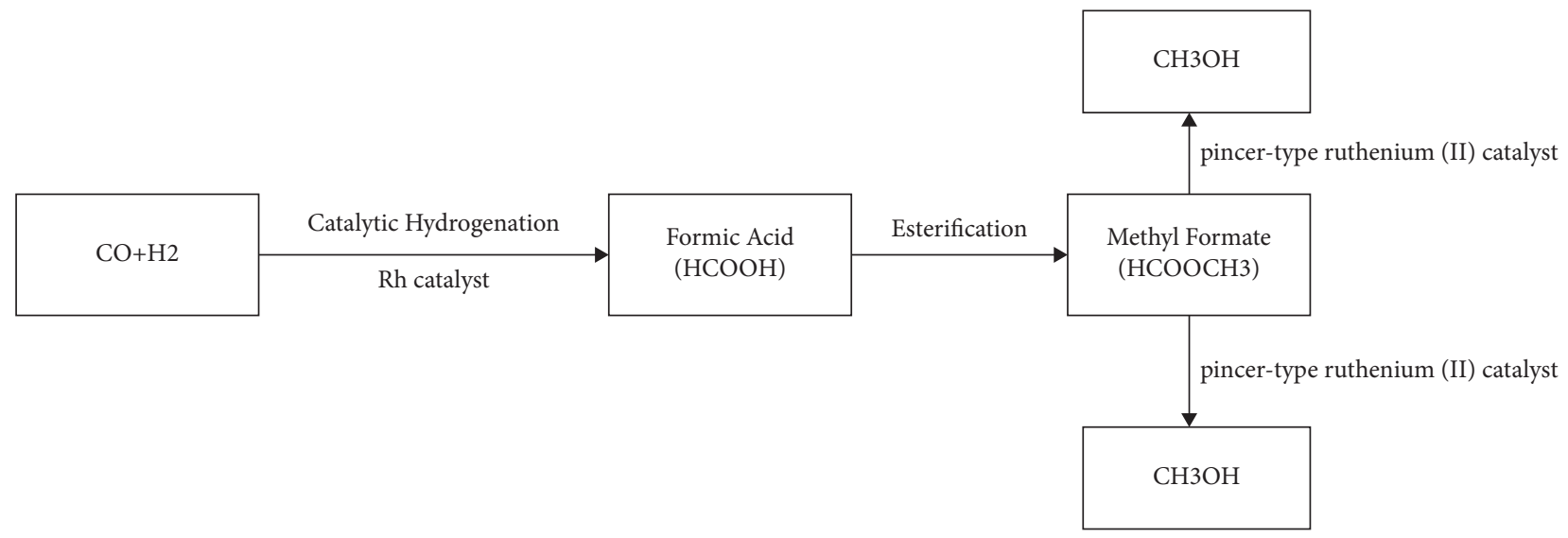

Figure 19: LTMS (syngas to methanol).
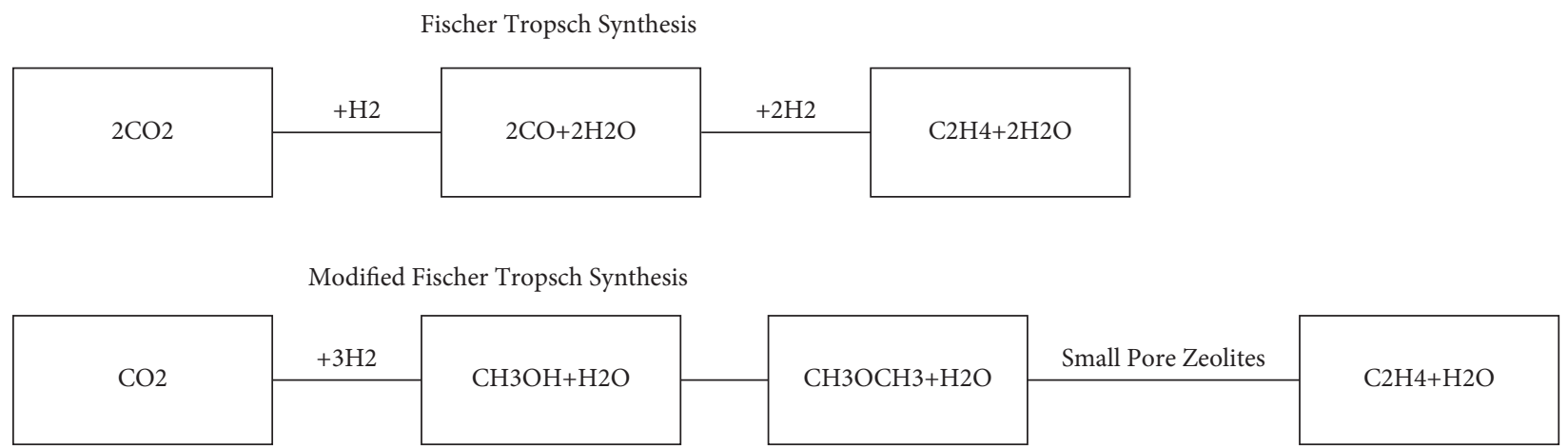

FIGURE 20: Fischer-Tropsch synthesis. Modified Fischer-Tropsch synthesis to find ethylene.

attention given its use in what is called power-to-gas technology and biogas upgrading. In this technology, surplus renewable energy is combined with $\mathrm{CO}_{2}$ and chemically converted to $\mathrm{CH}_{4}$, which can then be stored and delivered using the existing infrastructure involving natural gas. The Sabatier reaction, which is catalyzed by titania-supported ruthenium $\left(\mathrm{Ru} / \mathrm{TiO}_{2}\right)$, was carefully studied. Dry processing was used to create a variety of $\mathrm{Ru} / \mathrm{TiO}_{2}$ powder catalysts. At temperatures below $300^{\circ} \mathrm{C}, \mathrm{CO}_{2}$ was effectively hydrogenated to methane without the production of carbon monoxide (CO). It is worth noting that even after being immobilized in a three-dimensional framework, some of the
$\mathrm{Ru} / \mathrm{TiO}_{2}$ catalysts retained their chemical characteristics. Furthermore, the use of immobilized catalysts resulted in considerable reductions in both catalyst weight and temperature variations in the reactor.

(5) $\mathrm{CO}_{2}$ to Formic Acid. The production of $\mathrm{HCOOH}$ from carbon dioxide to store hydrogen in liquid form broadens the range of potential reactions for employing hydrogen derived from sources which are ultimately renewable and therefore the proportion of renewable energy in the chemical industry. There have been few instances of direct attempts to produce formic acid by direct $\mathrm{CO}_{2}$ 
electroreduction. Homogeneous catalysts have shown to be the most effective in converting $\mathrm{CO}_{2}$ to derivatives of formic acids, namely, formamides or formates. Homogenous complexes of $\mathrm{Fe}$ and Ir can be used for this conversion. Certain novel approaches have also been found out to be really effective as far as the amount of $\mathrm{HCOOH}$ which is produced. Nitrogen-doped titanium dioxide is used for both the reduction of $\mathrm{CO}_{2}$ to formic acid as well as the photo-oxidation of methyl orange.

The hydrogenation of formic acid was reported for the first time back in 1935, when a nickel-aluminium alloy called Raney Nickel was used. But the process parameters used were quite extreme. To avoid such extreme pressure and temperature, some different catalysts were put into use. Pdcomplex was then used just to find out that the process under this catalyst was thermodynamically unstable. Although reasonable formic acid conversion and yield have been achieved employing catalysts that are homogenous, separation of $\mathrm{HCOOH}$ from catalysts still poses as a challenge. As a result, developing a heterogeneous catalytic system is extremely desired. Ruthenium complex catalysts adsorbed on $\mathrm{SiO}_{2}$ are quite effective. The activated carbon supported ruthenium is a more appealing heterogeneous system. This approach has an upper hand over the other processes due to the use of toxic chemicals such as LAH, being totally eliminated, thereby making this procedure highly cost effective when compared to the conventional process [311].

In case of catalysts which use nanomaterials, the reduction of $\mathrm{CO}_{2}$ takes place in a different way.

(6) Photocatalytic Reduction of $\mathrm{CO}_{2}$. The scarcity of hydrocarbon fuel resources has encouraged study of photocatalytic reduction of $\mathrm{CO}_{2}$ into $\mathrm{HC}$-based fuels. This was a very appealing alternative for a sustainable and environmentally friendly path when it came to generating energy.

Through a proton-assisted multiple electron-transfer process, certain semiconductor oxide/sulfide nanomaterials such as $\mathrm{TiO}_{2}$ and $\mathrm{CdS}$ along with metal complexes, under the influence of the sun when suspended atop $\mathrm{CO}_{2}$-saturated water can produce methane, methanol, and formic acid. $\mathrm{Cu}$ nanoparticles produce methane, ethylene, and ethane when they are used as a cocatalyst with silicon carbide in the process of photocatalytic $\mathrm{CO}_{2}$ reduction.

It was found that supported core-shell $\mathrm{Au}$ nanoparticles ( $\mathrm{Au} @ \mathrm{SiO}_{2}$ ) which have sizes ranging from $8-25 \mathrm{~nm}$ are responsible for methanation of $\mathrm{CO}_{2}$ via the size-dependent localized surface plasmon resonance (LSPR) effect. 3D porous nanostructures of bismuth tungstate $\left(\mathrm{Bi}_{2} \mathrm{WO}_{6}\right) \mathrm{QDs}$, on the other hand, may selectively catalyse the photoreduction of $\mathrm{CO}_{2}$ to methanol when ethylene glycol is taken as the solvent and a solvothermal process happens [312].

It was revealed recently that $\mathrm{Zn}$ on top of a $\mathrm{Cu}-\mathrm{Ga}$-based LDH (layered double hydroxide) can be used as a coherent photocatalyst in the photocatalytic reduction of $\mathrm{CO}_{2}$ to $\mathrm{CH}_{3} \mathrm{OH}$. Moreover, Nile red, a well-known dye which is implanted on $\mathrm{TiO}_{2}$ electrode, was used in the photocatalytic reduction of $\mathrm{CO}_{2}$ to $\mathrm{CH}_{3} \mathrm{OH}$. As a byproduct of this process, oxygen is produced, simulating the photosynthetic path irrespective of whether or not $\mathrm{Pd}$ is present as an extra constituent of the electrode material [313].

A simple type of photocatalytic reduction over a homogeneous iridium catalyst was then discovered in which $\mathrm{CO}_{2}$ can be reduced to formic acid (FA) directly. Furthermore, formic acid can be selectively transformed into methanol in the presence of sulphuric acid by a disproportionation in high yield [314].

(7) Electrocatalytic Reduction of $\mathrm{CO}_{2}$. Two major parameters determine the selectivity of the product for electrocatalytic reduction of $\mathrm{CO}_{2}$ :

(1) Metallic nanoparticles size

(2) Dispersion rates on suitable supports

Metallic tin quantum particles which were present in graphene oxide nanosheets demonstrated extremely strong $\mathrm{CO}_{2}$ reduction electrocatalytic activity. Similarly, a high activity of electrocatalytic reduction of $\mathrm{CO}_{2}$ to $\mathrm{HC}$ fuels was seen when $\mathrm{Cu}$ nanoparticles held by faulty graphene nanosheets were tested [315].

Sometimes, for certain NPs such as Ag NPs which are suffused over MWCNTs, the case is different, as in, they selectively yield $\mathrm{CO}$ from $\mathrm{CO}_{2}$.

CNFs, CNTs, nanoporous carbon, or any given nanomaterial which is metal-free can be said to be used for the reduction of $\mathrm{CO}_{2}$ to one-carbon molecules such as methanol, formaldehyde, methane to name a few or two-carbon molecules such as ethanol or ethane) right after doping with atoms such as Boron, Nitrogen etc.

The abovementioned materials are opted more than metal-based catalyst systems when it comes to the electrochemical reduction of $\mathrm{CO}_{2}$ because of reasons like the flexibility of synthesis of carbon-based NMs from ubiquitously available resources, superb properties of the final product such as good surface area, and relatively higher resistance to acidic/basic conditions [315].

(8) Photoelectrochemical Reduction of $\mathrm{CO}_{2}$. The important criteria for obtaining high efficiency of these systems are complete control over the electron hole separation of the nanostructures. Moreover, the potential of the respective nanosystems going in place for the $\mathrm{CO} 2$ reduction. Since $\mathrm{Cu}$ has its own flexibility and novel capacity to reduce $\mathrm{CO}_{2}$ to $\mathrm{CO}$ via an electrochemical route, they have been investigated for photoelectrocatalytic reduction of $\mathrm{CO} 2$ to formate. This comes with an additional condition wherein the $\mathrm{Cu}$ NPs are coated on the $\mathrm{Co}_{3} \mathrm{O}_{4}$ nanotube arrays.

Different types of catalysts are used to convert $\mathrm{CO}_{2}$ to products which might be of great use in the future (Table 13).

It was only recently discovered that heterophase $\mathrm{Au}$ nanorods (NRs) have improved catalytic selectivity and activity in $\mathrm{CO}_{2} \mathrm{RR}$. The FCC-2H-FCC heterophase structure of $\mathrm{Au} \mathrm{NR}$ is well established. It displayed exceptional catalytic efficacy toward $\mathrm{CO}$ generation when employed as a catalyst for $\mathrm{CO}_{2} \mathrm{RR}$. Amorphous nanomaterials are also efficient $\mathrm{CO}_{2} \mathrm{RR}$ catalysts. As an example, amorphous $\mathrm{Cu}$ NPs were produced and subsequently employed as a $\mathrm{CO}_{2} \mathrm{RR}$ 
TABLE 13: Catalysts employed in copious types of carbon dioxide reduction.

\begin{tabular}{|c|c|c|c|c|}
\hline Sr. no. & Catalyst & Product & Type of $\mathrm{CO}_{2}$ reduction & Reference \\
\hline 1 & $\mathrm{Pt} \mathrm{NPs} / \mathrm{TiO}_{2}$ & $\mathrm{CH}_{4}$ & Photoelectrochemical reduction & [316] \\
\hline 2 & $\mathrm{Cu}_{2} \mathrm{O}$ & $\mathrm{CO}$ & Photoelectrochemical reduction & [317] \\
\hline 3 & Ultrathin metallic colayers & $\mathrm{CO}$ & Photoelectrochemical reduction & [317] \\
\hline 4 & $\mathrm{Pd} / \mathrm{Ni}$ & HCOO- & Photoelectrochemical reduction & [318] \\
\hline 5 & Coporphyrin & $\mathrm{HCO}_{2} \mathrm{H}$ & Photoelectrochemical reduction & [241] \\
\hline 6 & $\mathrm{~g}-\mathrm{C}_{3} \mathrm{~N}_{4}$ & $\mathrm{HCO}_{2} \mathrm{H}$ & Photoelectrochemical reduction & [241] \\
\hline 7 & $\mathrm{Cu}$ NPs & $\mathrm{C}_{2} \mathrm{H}_{4}$ & Photoelectrochemical reduction & {$[319]$} \\
\hline 8 & CdS & $\mathrm{HCHO}$ & Photocatalytic reduction & [319] \\
\hline 9 & $\mathrm{ZnO}$ & $\mathrm{CH}_{2} \mathrm{O}_{2}$ & Photocatalytic reduction & [319] \\
\hline 10 & $\mathrm{ZnS}$ & $\mathrm{CH}_{3} \mathrm{OH}$ & Photocatalytic reduction & [319] \\
\hline 11 & $\mathrm{SrTiO}_{3}$ & $\mathrm{CH}_{4}$ & Photocatalytic reduction & {$[320]$} \\
\hline 12 & $\mathrm{Cu} \mathrm{NPs} / \mathrm{SiC}$ & $\mathrm{CH}_{4}$ & Photocatalytic reduction & {$[320]$} \\
\hline 13 & $\mathrm{Cu} \mathrm{NPs} / \mathrm{SiC}$ & $\mathrm{C}_{2} \mathrm{H}_{2}$ & Photocatalytic reduction & {$[320]$} \\
\hline 14 & $\mathrm{Cu} \mathrm{NPs} / \mathrm{SiC}$ & $\mathrm{C}_{2} \mathrm{H}_{4}$ & Photocatalytic reduction & {$[320]$} \\
\hline 15 & $\mathrm{Au} / \mathrm{SiO}_{2}$ & $\mathrm{CH}_{4}$ & Photocatalytic reduction & {$[321]$} \\
\hline 16 & Bi2WO6 quantum dots & $\mathrm{CH}_{3} \mathrm{OH}$ & Photocatalytic reduction & {$[321]$} \\
\hline 17 & $\mathrm{Zn} / \mathrm{Cu}-\mathrm{Ga}$ & $\mathrm{CH}_{3} \mathrm{OH}$ & Photocatalytic reduction & {$[321]$} \\
\hline 18 & Nile Red ( $\mathrm{TiO}_{2}$ electrode) & $\mathrm{CH}_{3} \mathrm{OH}$ & Photocatalytic reduction & [321] \\
\hline 19 & Homogenous Ir catalyst & $\mathrm{CH}_{2} \mathrm{O}_{2}$ & Photocatalytic reduction & {$[321]$} \\
\hline 20 & $\mathrm{Cu}-\mathrm{Pd}$ alloys/organic polymer & $\mathrm{CH}_{4}$ & Electrocatalytic reduction & {$[321]$} \\
\hline 21 & Graphene oxide nanosheets & $\mathrm{CH}_{4}$ & Electrocatalytic reduction & {$[322]$} \\
\hline 22 & $\mathrm{Cu}$ NPs/Graphene & $\mathrm{CO}$ & Electrocatalytic reduction & {$[322]$} \\
\hline 23 & Ag NPs/MWCNTs & $\mathrm{CO}$ & Electrocatalytic reduction & [322] \\
\hline 24 & $\mathrm{CNT}$ & $\mathrm{HCHO}$ & Electrocatalytic reduction & {$[322]$} \\
\hline 25 & Nanoporous carbon & $\mathrm{CH}_{2} \mathrm{O}_{2}$ & Electrocatalytic reduction & [323] \\
\hline
\end{tabular}

catalyst. It was proposed that the higher $\mathrm{CO}_{2}$ adsorption ability and increased electrochemically active surface area of the amorphous $\mathrm{Cu}$ NPs contribute to their superior $\mathrm{CO}_{2} \mathrm{RR}$ performance. Furthermore, long-term measurements demonstrated that amorphous $\mathrm{Cu}$ NPs have superior electrocatalytic endurance than $\mathrm{Cu}$ NPs [324].

4.2.2. Water Splitting. Water splitting is one of the more important processes involved to fabricate hydrogen. In case of mechanisms involving catalysts that are not associated with nanomaterials:

Given the global economy's expansion, predictions are that the consumption of energy will continue to rise significantly in the times to come, and $\mathrm{H}_{2}$ which acts as an alternative fuel with a nature actively used to promote sustainable goals, is recommended as the eventual and perfect fuel for civilization in the future. Water splitting and fuel cells are two energy conversion processes that may be used to generate and use $\mathrm{H}_{2}$ in a sustainable manner. The hydrogen produced by this mechanism may be powered by renewable energy sources such as solar energy or wind energy; $\mathrm{H}_{2}$ used in fuel cells also actively releases electric energy. Water splitting, an innovative technique for energy conversion, which is powered by renewable energy, is gaining popularity for producing clean hydrogen fuel from water. To complete the energy transfer process, two essential processes, hydrogen evolution reaction (HER) and oxygen evolution reaction (OER), are involved. For this process, cost-effective catalysts are also necessary. Iron nickel combined (FeNi) catalysts are particularly favourable, owing to the FeNi synergistic relationship which is associated with boosting basic chemical reactions. It is noted that, when compared to other conventional electrocatalysts, FeNi catalysts perform better in catalysis of both HER and OER due to the presence of the synergistic effect that takes place between Fe and Ni. Nickel and iron are usually found together on Earth, and even small quantities of iron in the electrolyte can significantly improve the HER and OER performance of Ni-based catalysts. Much research has been conducted to investigate the synergistic impact of $\mathrm{Fe}$ and $\mathrm{Ni}$ in the FeNi system. Given the fact that there exists a strong intrinsic activity of iron-nickel catalysts for water splitting reactions, future research efforts may be better directed toward exploring catalysts in actual devices, most likely by combining with commercial applications, rather than new nano catalysts screening [325].

Albeit there exist quite a few challenges for sunlightemployed water splitting, several methods were still devised and they grew over time. Copper oxide layered over titanium dioxide was found to be a photocatalyst with a catalytic activity which was increasing as the temperature increased. Glycerol can be used as a sacrificial agent to enhance the catalytic activity of the latter. Light-induced heating was carried out constantly to boost the catalytic performance. Ruthenium anatase catalysts were then fabricated for a photo thermal production of hydrogen via water splitting. This process used $\mathrm{CH}_{3} \mathrm{OH}$ as a sacrificial agent. Nickel combined with $\mathrm{TiO}_{2}$ also showed a similar kind of a process as ruthenium anatase catalyst. The catalytic performance and activity increased as the temperature of the reaction kept increasing [54].

Nickel complexes' design and catalytic characteristics for water splitting have been explored in terms of their 
molecular catalysis. Nickel-based catalysts have garnered a lot of interest in the HER for both electrocatalytic applications as well as photocatalytic applications. The rational design may entail the identification of suitable donors for ligand coordination and functionalization. Limited nickel type molecular catalysts have been described in the OER, notably in photocatalysis. More efforts should be made to create nickel catalysts with an enhanced activity in order to accomplish total water splitting. During the last several decades, remarkable progress has been made in the creation of nickel-based molecular catalysts with outstanding electro/ photocatalytic activity for all the three reactions: hydrogen evolution, oxygen evolution, and carbon dioxide reduction. However, the proper utilisation of these catalysts for electrocatalytic and photocatalytic production of hydrogen or any fuel is still a long way off. Significant efforts will also be required in the times to come to enhance and boost the catalytic activity of these systems [57].

In case of those catalysts which use nanomaterials and their superb properties for the enhancement of the catalytic activity.

The rapid growth of the world's population has resulted in an ever-increasing need for energy. The search for sustainable energy sources is becoming a pressing problem. When burnt in oxygen, hydrogen is one such fuel that emits no pollutants. It is a potential renewable fuel that promises great performance in various applications concerning copious departments from cars to electronic gadgets to even propulsion of spacecrafts. Techniques for separating hydrogen from HCs and other organic compounds which contain hydrogen are shown. Water splitting is considered to be one of the most fascinating methods of hydrogen extraction. Principle for water splitting has been demonstrated (Figure 21).

Semiconductors are unique in their ability to serve as photocatalysts. As a result, it is critical in triggering the reduction and oxidation reactions which may take place in the presence of light. These photocatalyst electrodes may capture light, which provides the energy for reactions, and an externally supplied electric/chemical bias gives the extra voltage necessary to carry out the process. This externally supplied bias compensates for the sluggish kinetics and supplies enough voltage for the PEFC to control the reaction at their respective process parameters.

There are different things on which the PEC water splitting depends. The effectiveness of water splitting based on PECs is primarily determined by their orderliness, homogeneity, and morphology. When compared to amorphous materials, highly ordered crystalline materials perform significantly better. Temperature is an important factor in increasing PEC efficiency. Experiments are typically carried out at high temperatures. According to one study, low temperature treatments enhance PEC efficiency. Larger particles have band bending characteristics, allowing for better charge extraction at the electrode-electrolyte interface. As a result, bigger cocatalysts are more suited for improved PEC performance. Strategies for enhancing hydrogen evolution include expanding active sites or boosting the electrical conductivity. To get going with total water splitting in a more straightforward manner, exposure of active sites, electron transport capabilities, and gaseous product release should be improved. Plasma treatments and layer thickness reduction can be used to increase the active site. Doping can be used to improve electrical conductivity.

Nanoscale diameters are similar to carrier scattering lengths, resulting in a substantial reduction in scattering rate and increased carrier collecting efficiency. Because of the rise in oscillator strength, nanomaterials have high absorption coefficients, allowing for great conversion efficiency. Nanoparticles are useful for photocatalysts because their mass transfer is rapid and they absorb more light. The nanoparticles can be used in increasing the photocatalytic activity by being coated on the electrode. This can also be done by having the nanoparticle suspended in the reaction solution. The metal nanoparticles were localised at the surface between semiconductor and the electrolyte where they improved water splitting efficiency.

Different nanomaterials were tested for water splitting, and almost always had a good result. Controlling and modifying the band gap levels of energy can change the electrical characteristics of NMs. Nanomaterials in semiconductors lower the band gap, allowing for more absorption of solar light. Given the unique characteristics of nanomaterials, there are several possibilities for designing systems with high photocatalytic effectivity for splitting of water utilising sunlight [326].

4.2.3. Environmental Treatment. VOC abatement in this generation for treating the environment has become necessary nowadays. VOCs tend to exist in air or water to pollute the same. It is highly desirable to create a technique for catalytic degradation that is both sustainable and efficient, and photothermocatalysis appears to be a viable option owing to the synergistic use of both light and heat from solar energy for pollution clean-up. With the combination of UV and NIR spectra, photothermocatalysis does the job of enhancing the abatement of VOC. When bombarded with the whole solar spectrum and IR light, Ce and Ni-based catalysts demonstrate exceptionally high catalytic activity for the oxidation of VOCs [54].

VOCs are reduced using Au-based catalysts that are either nanomaterial coupled or not. Its catalytic activity is dependent on the oxide support and how they are prepared, showing that $\mathrm{Au}$ and the support have synergistic effects. Indeed, such interactions have gathered awareness lately, with various sorts of interchange being disclosed based on the metals and supports used and how they were prepared. By altering the characteristics of the catalysts, strong metalsupport interactions (SMS) and vital oxide-support interactions (SOS) can dramatically affect catalytic activity (size, morphology, and electronic states). The addition of $\mathrm{NaBH} 4$ to Pt improves its catalytic activity in the oxidation of benzene and formaldehyde. At standard temperature, $\mathrm{CO}$ and $\mathrm{HCHO}$ can be degraded catalytically into $\mathrm{CO}_{2}$, while most contaminants with aromatic ring(s) cannot. As a result, photocatalytic degradation of such pollutants (such as toluene) has gotten much interest, with the ultimate goal of 


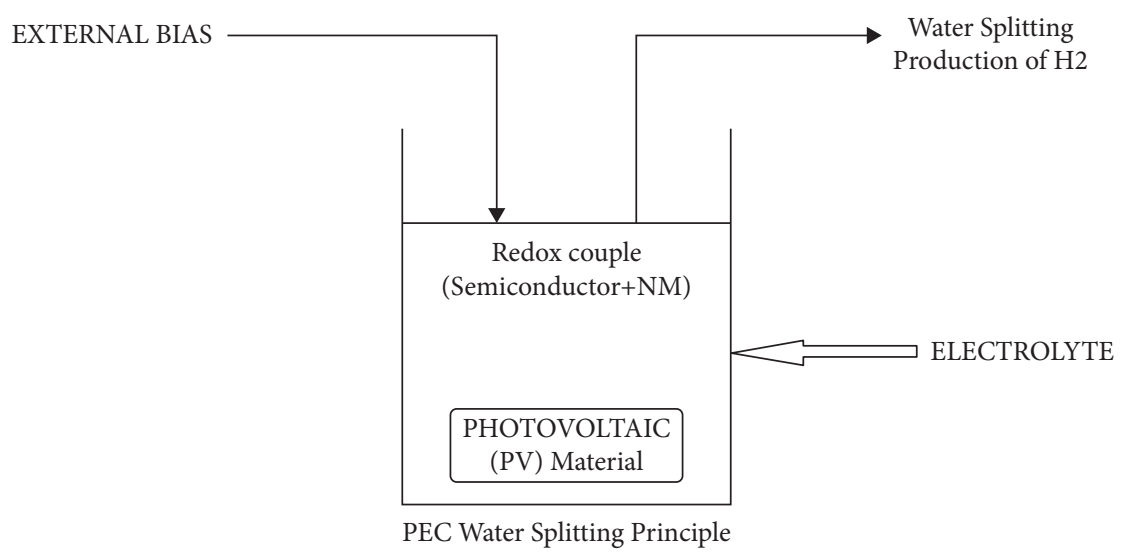

FIGURE 21: Principle by which water splitting takes place.

complete pollutant elimination. Photocatalysts possessing high visible activity are required for such applications to use solar energy effectively [60].

Due to rising worries currently about the change of climate, impact of ecology, and human health, an enormous amount of effort is taken to restrict the discharge of contaminants. The development of robust and highly effective catalysts for environmental clean-up has been a significant focus of this study. Noble metal catalysts are typically highly active; however, they come at a high cost and are susceptible to contamination poisoning. Transition metal oxides with high low-temperature catalytic activity are being investigated as inexpensive replacements to conventionally available precious catalysts made of Platinum, Pd, etc. Copper and its oxides have gotten much attention lately. $\mathrm{Cu}$ is a transition metal with unique physical and chemical characteristics. Because of their three distinct oxidation states, $\mathrm{Cu}$-based materials may stimulate a wide range of reactions. Copper oxides such as $\mathrm{CuO}$ and $\mathrm{Cu}_{2} \mathrm{O}$ are used widely as catalysts in NOx's selective catalytic reduction (SCR) utilizing $\mathrm{NH}_{3}$, catalytic combustion of VOCs, etc. Copper oxides play a significant role in reducing $\mathrm{CO}$ emissions from the atmosphere, thereby saving the environment. $\mathrm{Cu}-\mathrm{Mn}$ based oxides are developed as effective catalysts for $\mathrm{CO}$ oxidation at low temperatures. The increased activity for $\mathrm{CO}$ oxidation is due to the high surface area and large pore volume of the $\mathrm{Cu}-\mathrm{Mn}$ bimetallic catalyst.

Due to the reduction of 4 to 3 electrons in the redox mechanism of $\mathrm{Ce}$, which may transmit electrons and further reduce to form $\mathrm{Cu}^{2+}$ or $\mathrm{Cu}^{+}$, cerium oxide in contact with copper ion $\left(\mathrm{Cu}^{2+}\right)$ promotes the production of $\mathrm{Cu}^{+}$. This improves $\mathrm{CO}$ adsorption, resulting in high catalytic activity. $\mathrm{Cu}_{2} \mathrm{O}$ has a good oxidizing $\mathrm{CO}$ at low temperatures, but it has poor stability as far as the thermal and hydrothermal parameters are concerned, limiting its commercial use.

Tang et al. recently used the supercritical antisolvent precipitation technique to make nanocrystalline copper manganese oxide catalysts with catalytic activity equal to more than twice the traditionally produced catalysts for oxidation of CO. The nanocrystalline and homogenous character of the produced copper manganese oxides was linked. In catalysis, the reduction of ecologically hazardous
$\mathrm{NO}_{x}$ compounds produced remains a difficult challenge, with SCR utilizing $\mathrm{NH}_{3}$ being the most commonly employed method. The conventional catalyst for commercial SCR of $\mathrm{NO}_{x}$ compounds utilizing $\mathrm{NH}_{3}$ is vanadium pentoxide placed atop titanium dioxide. $\mathrm{Cu}$-based catalysts supported on zeolites have lately emerged as appealing options given their eco-friendly properties and excellent SCR performance across a wide temperature range alongside having good selectivity, even after many attempts [60].

CuO's morphology and form have been demonstrated to impact $\mathrm{CH}_{x}$ oxidation activity significantly. In the catalytic combustion of volatile organic compounds, mixed metal oxides have been widely used. Toluene combustion has been produced and tested using copper oxides [327].

As far as wastewater treatment was concerned, Fentonbased processes were unique in their ability to degrade the organic pollutants in water. The Fenton process is an advanced oxidation process that uses $\mathrm{Fe}^{+3}$ ions and $\mathrm{H}_{2} \mathrm{O}_{2}$ to generate hydroxyl radicals, which combine with the reactant, which is copper, to decompose the organic pollutants present inside the wastewater, thereby treating it. Copper has also demonstrated Fenton-like solid catalytic activity when placed on different supports, improved the dispersion of $\mathrm{Cu}$ active sites and supported copper oxides were produced. Copper placed over aluminum oxide creates a heterogeneous catalyst with high efficiency and operational stability. In the presence of excess $\mathrm{NaBH}_{4}$, Ghosh et al. filled $\mathrm{Cu}$ nanoparticles with SBA15, a kind of silica for removing different dyes, such as 4-nitrophenol and combinations of dyes. This material's catalytic solid activity aided the breakdown of dyes. On the other hand, mesoporous copper ferrite (mesoCuFe $\mathrm{O}_{4}$ ) was discovered to have a high specific area and a well-ordered lattice, resulting in an increased number of active sites and facilitating the adsorption and mass transfer of giant molecules that might contaminate water [328].

Similarly, catalysts based on copper have also been extensively studied in solid waste treatment, with encouraging results. Several oxides are efficient for combusting soot catalytically, including mixed oxides and perovskite-like oxides [61]. The application of catalysts has been displayed (Table 14). 
TABle 14: Catalysts and its degrading effect on substrate.

\begin{tabular}{|c|c|c|c|c|}
\hline Sr. no. & Catalyst & Reduction/degradation of & Source/effects & Reference \\
\hline 1 & Carbon dots/ $/ \mathrm{TiO}_{2}$ & $\mathrm{Cr}(\mathrm{VI})$ & Toxic groundwater & [329] \\
\hline 2 & FGT (F-doped $\mathrm{GO} / \mathrm{TiO}_{2}$ ) & Bromate & Carcinogenicity of inorganic pollutant & [330] \\
\hline 3 & Carbon nanofiber/titanate NT & Acetaldehyde & Common workplace contaminant & 0] \\
\hline 4 & CQD-modified- $\mathrm{Bi}_{2} \mathrm{WO}_{6}$ nanosheets (CBW) & Methyl orange (MO) & pollutant & {$[331]$} \\
\hline 5 & CQD-modified- $\mathrm{Bi}_{2} \mathrm{WO}_{6}$ nanosheets (CBW) & BPA (bisphenol A) & Wastewater treatment plant byproduct & [331] \\
\hline
\end{tabular}

When photocatalysts enriched with carbon nanomaterials are bombarded with light, electrons are synthesized in the conduction band, and holes are synthesized in the valence band. Electrons can be delivered to electron acceptors to react with molecular $\mathrm{O}_{2}$ to form superoxide radicals that can oxidize organic contaminants, thereby treating the environment. Holes with significant oxidizing properties participate directly in oxidation via photocatalytic route or bond with the $\mathrm{OH}^{-}$ions which were adsorbed to create $\mathrm{OH}$ radicals.

When it comes to degrading organic contaminants, CNMs are an excellent choice. The photocatalytic performance of C60 fullerene-modified ZnAlTi-LDO (layered double oxide) on the breakdown of bisphenol A was excellent. The electrical link between MWCNTs and $\mathrm{TiO}_{2}$ facilitates photoinduced electron transfer from $\mathrm{TiO}_{2}$ to MWCNTs, resulting in increased catalytic activity. This stems from the fact that MWCNT-doped $\mathrm{TiO}_{2}$ films have been touted as effective photocatalytic degraders of P-nitrophenol, an environmental hazard. A high MWCNT content would alter the film's transparency and prevent $\mathrm{TiO}_{2}$ from interacting with light, lowering photocatalytic activity. Typically, platinum supported on carbon is utilized for this purpose. Platinum is a highly costly catalyst, as is evident. Apart from the expensiveness, PEFCs have poor performance durability, primarily due to catalyst migration.

Lately, metal porphyrins are coming out to be one of the most attractive non-precious cathode catalysts, with Co and Fe porphyrins being the most favorable precursors. There has been a protracted search for catalysts other than precious metal catalysts for the PEFC cathode, which has been ongoing for quite some time. There have been few materials exhibiting potential ORR activity in either primary or fuelcell investigations and almost none with high efficiency. Even the most exhaustively researched metal-porphyrins lack durability under real-world fuel-cell operating circumstances, reinforcing the necessity for additional research into non-precious cathode electrocatalysts for PEFCs.

After almost half a century of hard work toward researching the catalytic activities of ORR catalysts, a new segment of catalysts without metals was discovered as an alternative to the conventional ORR, which was found to have low cost as well as increased efficiency. In particular, VANCNT (vertically aligned nitrogen carbon nanotube) had a metal-free design, turned out to have almost three times stability and durability compared to Pt catalysts. One more significant advantage was the latter being free from methanol crossover effects and $\mathrm{CO}$ poisoning, which existed in platinum catalysts [332].

For inorganic pollution remediation, a photocatalytic reduction over carbon nanomaterials is shown to be effective. Bromate, a common inorganic contaminant produced as a byproduct of the oxidation process used to purify water, was highly toxic. A lower $\mathrm{pH}$ was found to be quite beneficial for bromate reduction in the photocatalytic process because of its good effect on bromate adsorption. By generating low harmful analog ions, the photoreduction process is an effective way to remove the virulent materials present inside the heavy metal ions.

In the case of photocatalytic disinfection, carbon nanomaterials serve three unique but crucial roles. To begin with, they serve as a scaffold to prevent photocatalyst aggregation in the composites generated. Second, conductive carbon nanomaterials served as an acceptor or donor of electrons, improving charge carrier separation and promoting OER to create $\mathrm{H}_{2} \mathrm{O}_{2}$. Finally, chemical connections between CNMs and photocatalysts can help boost photocatalytic disinfection performance [259].

(1) Remediation of Wastewater. The growing population increases the demand and supply levels of the amount of water available for drinking. Over 1.1 billion people are projected to be without adequate drinking water. The continual pollution of freshwater by range contaminants is a severe problem in the water supply chain. These problems can be alleviated by treating wastewater and drinking water. Traditional treatment procedures, on the other hand, are unable to eliminate new pollutants and fulfill stringent water quality criteria.

Furthermore, current wastewater treatment methods have numerous disadvantages, including high energy consumption, inadequate pollutant removal, and the production of hazardous sludge. Biological wastewater treatment is extensively used, although it is often sluggish, restricted by the presence of nonbiodegradable pollutants, and can sometimes induce microorganism toxicity owing to toxic chemicals. Physical methods like filtering can remove pollutants by converting one phase to another, producing a hazardous and difficult-to-dispose-of sludge. A range of efficient, ecofriendly, and cost-effective nanoparticles with distinct capabilities for possible decontamination of industrial effluents have been created for wastewater treatment applications.

As far as the nanomaterials concerning wastewater treatment are concerned, there are mainly 3 types in which they could be divided: 
(1) Numerous studies on the alleviation of contaminants from wastewater using nano-adsorbent materials have recently been published in nano-adsorption technology. Chemically active elements and those elements having a good adsorption capacity on the surface of the NM can be used to make nano-adsorbents. Activated carbon and metal oxides are among the many materials that are utilized in the creation of nano-adsorbents.

(2) Second, scientists have paid close attention to nanomaterials, nanocatalysts to be precise, such as metal oxides and semiconductors for the establishment of wastewater treatment systems. For the degeneration of pollutants in wastewater, several types of nanocatalysts are used, such as electrocatalysts and Fenton-based catalysts, for enhancing the chemical oxidation of organic pollutants.

(3) Thirdly, nanomembranes have become a popular study area. Pressure-driven wastewater treatment has been shown to be suitable for increasing desired water quality. Nanofiltration (NF) is a form of membrane filtration that is widely used in industries for wastewater treatment due to its small pores and high effectiveness. Different types of nanomaterials can be used to create nanomembranes.

For the elimination of heavy metals from wastewater, nanoadsorbents are often employed. The most widely utilized nanoparticles for this purpose are carbon nanotubes and metal oxides. These nanoparticles have several distinguishing characteristics, including a large BET surface area, a high dispersion capacity, and are both economically and environmentally beneficial. However, secondary pollution will occur as a result of the smaller particle size and problems in separating them from the aqueous solution. This increases the bioavailability and mobility of heavy metals, resulting in environmental toxicity. Another issue for these nanoparticles is their costeffective reuse and regeneration. To address these problems, several researchers used modifications like multiwall carbon nanotubes (MWCNT). New nanoadsorbent trends, such as organic-inorganic hybrids, are a viable alternative for overcoming the limits of the nanoadsorption process.

Advances in catalytic wastewater treatment might benefit significantly from the use of nanoparticles. Due to their large bandgap energy, widely used metal oxide catalysts in photocatalysis have a limited demand for UV light. Workers may be exposed to UV radiation due to the industrial use of these materials, posing a severe health risk such as skin cancer and DNA mutation. Because it is necessary for the industry to generate high-quality water, there exists a clear need to fabricate durable materials and ways to address said issues. The usage of catalysts made of NMs has several restrictions in terms of process. To solve the difficulties associated with traditional catalysts, researchers are currently developing novel photocatalysts made of metal oxide or a combination of metals and semiconductor oxide. Pt is a frequently used catalyst in fuel cells; however, its application has several drawbacks that impact the electrocatalysis process.
The advantages of membrane filtering technology as far as the water treatment are concerned, efficient sterilization, and minimal plant area requirements are the major reasons for its use. In addition, compared to other treatment methods, it is very cost-effective and easy to implement. The abolishment of dyes and heavy metals can be accomplished with nanomembrane separation technology. Nanomembranes have a high ecological impact throughout their production process, which raises environmental issues. Another drawback is the fouling of the membrane, which is produced by organic molecules in $\mathrm{H}_{2} \mathrm{O}$ interacting with the hydrophobic membrane. Particle deposition on the membrane surface or within the membrane pores increases the risk of membrane fouling. Membrane fouling lowers water flux, necessitating a membrane clean-up operation using chemical or mechanical techniques or, in certain cases, a full membrane replacement. To address these issues, researchers are concentrating on covering the membrane with a polymer layer, such as polyvinyl alcohol. This layer is also designed to be hydrophilic. Furthermore, nanoparticles such as $\mathrm{TiO}_{2}$ can be introduced into membranes to improve their hydrophilicity and therefore reduce fouling [259].

4.2.4. Hydrogen Uptake. The increased dangers posed by global warming due to the use of fossil fuels necessitate the adoption of innovative techniques to exploit the limitless amount of energy available. Hydrogen is an energy carrier with great potential as a source of novel renewable energy. $\mathrm{H}_{2}$ is a handy and adaptable fuel that can readily be transformed into any given form of energy while emitting no hazardous pollutants. Because of their unique surface and bulk absorption properties, NMs hold great promise in hydrogen storage. Furthermore, NMs allow for the control of tailoring characteristics of the materials independent of their bulk equivalents. Moreover, they contribute to the development of lightweight systems of hydrogen storage with improved storage properties [333].

Steam reforming is the most often used technique for hydrogen absorption. However, the vast amount of $\mathrm{CO}_{2}$ emissions produced by steam reforming is a significant drawback. As a result, other techniques other than steam reforming have emerged in recent years. The current work uses heterogeneous nanocatalysts to create a low-cost, ecologically friendly method for producing hydrogen from glycerol.

The use of heterogeneous nanocatalysts to produce hydrogen from glycerol offers numerous benefits over traditional steam reforming:

(1) Removing the vaporization stages of water, reformed raw material, resulting in depletion of energy requirement

(2) Operating at lower temperatures, which prevents parallel breakdown processes of oxygenated compounds while boosting $\mathrm{CO}$ removal, allowing $\mathrm{H}_{2}$ generation and a low $\mathrm{CO}$ concentration in a onestage operation 
(3) Low operating pressure, allowing for the purification of $\mathrm{H}_{2}$ using adsorption

Noble metals were shown to be quite active when it came to generating hydrogen for the aqueous phase reforming of oxygenated HCs that are spread over various metal supports. However, as we all know, the exorbitant expense of noble metals in general, and their restricted supply in particular, prompted research into finding less costly catalysts for reforming purposes. As a result, nickel was chosen as the active phase in the current study. Furthermore, the structural features were extensively researched before making this selection. Nickel can be combined with the support of $\mathrm{Al}_{2} \mathrm{O}_{3}$ for the further enhancement of the catalytic activity, which allows more capture and production of hydrogen.

$\mathrm{MgO}$ is commonly utilized as a promoter for $\mathrm{Ni} / \mathrm{Al}_{2} \mathrm{O}_{3}$ catalysts because it improves nickel sintering stability. In the literature, the addition of $\mathrm{Al}_{2} \mathrm{O}_{3}$ to this catalyst was also reported to be an ingredient capable of increasing its stability. Aluminum oxide and magnesium oxide were mixed in various ratios to discover which one worked the best. It was discovered that mixed-oxide samples outperformed singleoxide samples in catalytic conversion. Ni/A2M1 (nickel/ $\mathrm{Al}_{2} \mathrm{O}_{3}: \mathrm{MgO}$ ratio of $2: 1 \mathrm{OR}$ nickel coated over two parts aluminum oxide and one part magnesium oxide). According to the findings, Ni/A2M1 catalysts also had greater reforming activity [325].

Microorganisms that use hydrogenase enzymes to consume hydrogen may provide a competitive alternative to traditional Pt catalysts. These metalloproteins catalyze the interconversion of $\mathrm{H}_{2}$ in both directions. Furthermore, when adsorbed on an electrode, they function without any extra applied voltage. Unfortunately, hydrogenases are oxygensensitive, which means they are destroyed and corroded in the presence of oxygen. Furthermore, their manufacturing in the active form is challenging to scale up to meet technological demand; thus, they are not exploited to their full economic potential. Since hydrogenases require just $\mathrm{Fe}$ and $\mathrm{Ni}$ centers for catalysis, the active sites were superb sources for developing synthetic catalysts that were much sustainable when compared to platinum [252].

There are various ways in which nanomaterials can participate to produce good results. Nanomaterials can act as dopants in advanced complex hydrides and borohydrides. Nanocatalyst doping also works perfectly to form a nanocrystalline form of $\mathrm{MgH}_{2}$ which will ultimately help for hydrogen uptake. On the other hand, carbonaceous materials are appealing options for $\mathrm{H}_{2}$ storage due to an integration of the ability to adsorb, high specific surface area, and pore-type structure.

4.2.5. Fuel Cells. For optimal utilization of expensive $\mathrm{Pt}$ catalysts, they are distributed as tiny pieces over conductive substrates possessing high surface area. In terms of both electrocatalytic activity and practical use of catalysts, $\mathrm{Pt}$ particles' size plays a crucial role in the kinetics of ORR, thereby helping fuel cell applications. Platinum with carbon support has a huge surface area and higher catalytic activity.
PEMFC (polymer exchange membrane fuel cell) model has been explained in (Figure 22).

Nanocatalysts have a considerable number of applications in the fields of science and technology due to their unique physical and surface properties. The different structural modifications produce nanocatalysts with flat $\mathrm{sp}^{3}$ hybridization, curved $\mathrm{sp}^{2}$ hybridization, and allotrope with $\mathrm{sp}^{3}$ hybridization. This specific hybridization, along with the presence of hexagonal substructure in a centered cylindrical plane in an axial alignment, provides nanotubes its unique catalytic features over nanofibers, with varying fibrous/tubular structure, resistivity to acidity and basicity, electrical conductance, exterior surface area, etc. Additionally, it is supported by the edge structures in its microstructure, which acts as a catalyst precursor anchoring site. These edge sites undergo an unsaturated coordination configuration, supported by the restrictive character of electrons inside the substructural dimensions of other particles, resulting in the size effect and the properties of the metal support provided for the catalyst, resulting in a support effect [334]. This was studied by analyzing the hydrocracking characteristics of the nanocatalyst material based on the amount of toluene and benzene formed in the process. For example, take the case of titanium, aluminum, silicon, and magnesium oxides, which were used as metal supports for the catalytic hydrocracking of diphenylmethane. The efficiency rate of each of these metal support decreases in the following manner:

$$
\mathrm{TiO}_{2}>\mathrm{SiO}_{2}>\mathrm{Al}_{2} \mathrm{O}_{3}>\mathrm{MgO} \text {. }
$$

The titanium dioxide-supported catalyst provided maximum hydrocracking activity, while the magnesium oxide-supported catalyst provided zero conversion. The selectivity nature for catalyst supported over the oxides of silicon and aluminum was observed to be really high [334]. Nevertheless, these metal supports showcased deficient hydrocracking activity, even though they possessed high external reaction surface area. During the hydrocracking process of 1-methylnapthelene, the rate of activity of the oxides of metal supports (titanium, silicon, aluminium, and magnesium) followed the order:

$$
\mathrm{Al}_{2} \mathrm{O}_{3}>\mathrm{TiO}_{2}>\mathrm{MgO}>\mathrm{SiO}_{2} .
$$

During the reaction mentioned above, the highest hydrocracking activity was observed in the aluminum dioxide-supported catalyst compared to the rest of the oxides. However, during the hydrocracking reaction of diphenylmethane, silicon dioxide-supported catalyst produced the highest hydrogenated products compared to the corresponding metal oxide-support catalyst of aluminum [334]. The metal-support interaction stabilized the atoms present in the metal dispersed during the reaction process during these reactions. Through structural engineering and altering the properties of the interface, the nanoframework of the carbon atoms can be adjusted with respect to its primary and secondary nanostructures. This provides new possibilities for tuning the properties of the external surface of carbon nanofiber and the supporting metal nanoparticle. Due to the 


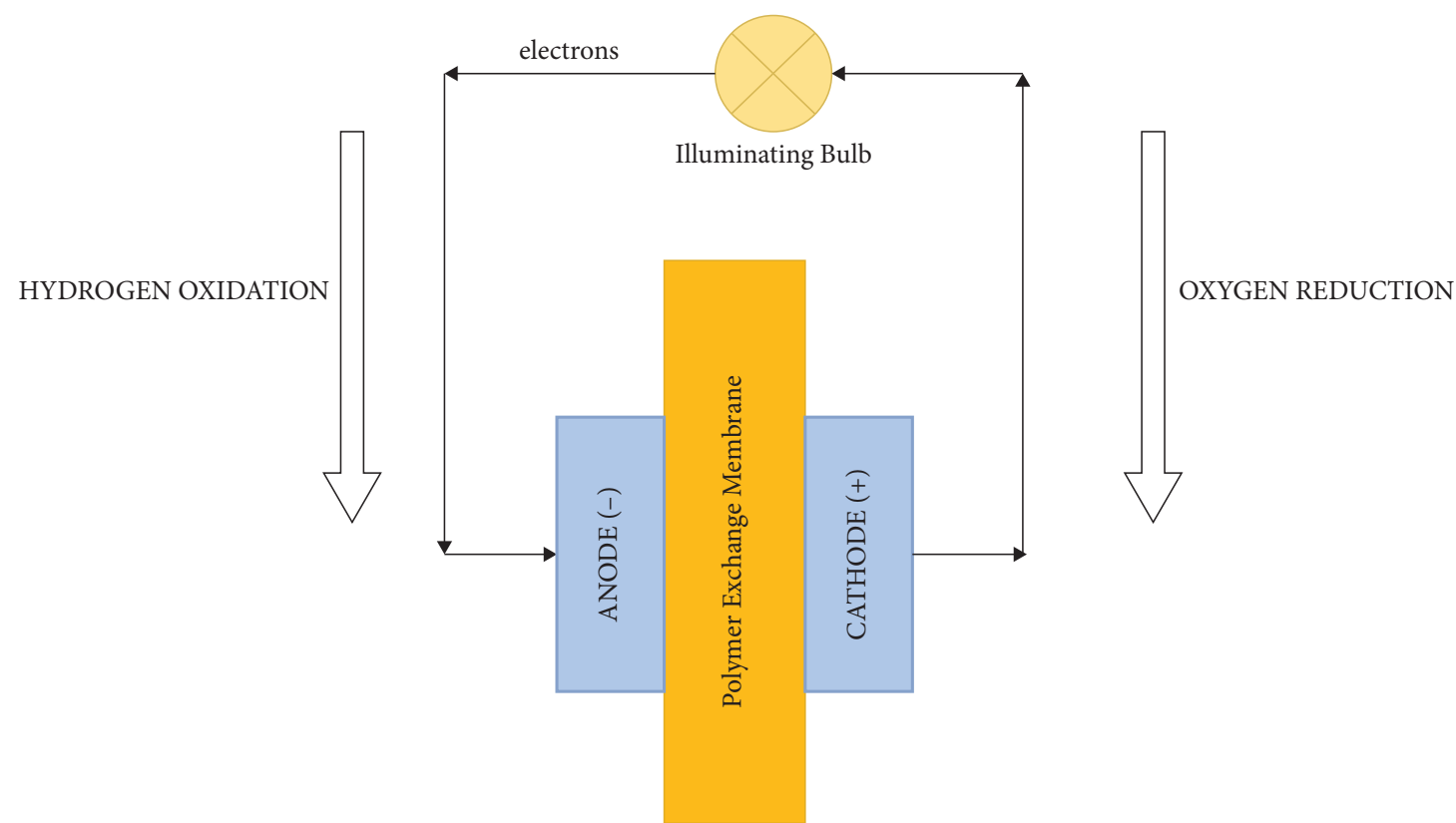

FIgURE 22: Reactions (oxidation and reduction) taking place in the PEMFC (polymer exchange membrane fuel cell). Illuminating bulb shows that the current is flowing through the circuit.

large facial area and increased electrothermal conductivity in its crystalline structure, CNTs and CNFs offer a better catalytic activity than traditional catalysts such as alumina and silica.

\subsubsection{Application of Dimensional Variation in Electrocatalysis.} Fundamental developments in electrocatalysis, which tend to pose a very significant impact on the technological advancement of fuel-cell in order to mitigate the effects of global warming and the limited existence of fossil fuels, are possible easily thanks to nanomaterials. The combination of superb properties possessed by these materials has piqued interest in nanostructured materials in recent years. Not only do they lower the energy barrier when they act as electrocatalysts but they also improve the charge transfer of electrons happening on the specific surface region. Generally, they are divided into different groups such as wires, docs, and sheets, but based on its confinement, they are divided into 4 categories: zero-dimensional (spherical or nearly spherical shapes), one-dimensional (long length nanowire or nanotubes), two-dimensional (well-aligned sheet-like structure called nanosheets or nanoplates), and three-dimensional (3D). Each dimension has its impact and advantages. Even though several signs of progress have been claimed for morphologically enhancing the nanomaterials, the recent novel approaches have slightly been insufficient. However, it is believed that, in the times to come, the production of nanostructured electrocatalysts is bound to increase, along with the fulfillment of the cost being reduced drastically. This will make it more appealing to curbing environmental issues and addressing global energy levels [152].

Two reliable approaches, namely, bottom-up and top-down approaches, have emerged to be ideal for their potential to synthesize nanomaterials with the asked requirements. The bottom-up method is applied based on the information accumulated by atom and molecule by molecule. Physiochemical methods may be used to combine basic structures into a larger stable structure. Atomic deposition causes atoms, molecules, and clusters to self-assemble. By self-assembly, a single layer of these molecular clusters is informed, for example, quantum dots. On the other hand, top-down methods involve breaking material of larger size into smaller parts with the help of mechanical forms of energy, mainly mechanical, chemical, or other energy. Any bulk content is taken in the top-down method, subjected to carving and cutting before the desired shape is reached. Several methods are used to convert bulk materials to nanomaterials, such as ball milling, electrospinning, sputtering, and etching, to name a few [152].

In the case of zero-dimensional nanomaterials, a peculiar morphology is followed. Because of their excellent performance in cathode oxygen reduction reactions (ORRs) and anode fuel oxidation reactions, platinum-based electrocatalyst materials have gained a lot of attention as an efficient catalyst in fuel-cell to monitor the shape of $0 \mathrm{D}$ nanostructures. Over the years, it was found that metal alloy catalysts can be incorporated with the metal being copper, thereby controlling the binding energy and reducing the use of the expensive. Moreover, the hydrogen atoms which were adsorbed on the surface of palladium nanomaterials can be utilized as a reducing agent to enhance reaction yield by reducing copper, thereby forming thin copper layers just to be replaced by platinum to form a platinum-palladium catalytic core [152].

In the case of $1 \mathrm{D}$ nanomaterials, they rise quite smoothly against 0 -D materials primarily due to their enhanced anisotropy, unique structure, and superb electron transport features. Many of the problems with 0D-based 
electrocatalysts can be solved with this technology. As a result, $1 \mathrm{D}$ nanostructures have made significant progress in electrocatalysis, with improved electrocatalytic behavior, utilization performance, and durability. The galvanic replacement reaction is the go-to mechanism where high noble ions of metal replace a less noble nanowire via redox mechanism due to the difference in their reduction potentials. A galvanic substitution reaction involving silver nanowires and a precursor of platinum may be used to make platinum nanotubes. The hydrothermal treatment of polycrystalline platinum nanotubes prepared by the abovementioned galvanic replacement reaction yielded single-crystalline platinum nanotubes containing dominant facets. In comparison with polycrystalline platinum nanotubes, the single-crystalline platinum nanotubes produced had better methanol oxidation reactivity and oxygen reduction reaction stability [152].

The characteristic structural dimensions and the electronic features of the catalyst surface provide the $2 \mathrm{D}$ nanomaterials with several catalytic properties. Since $2 \mathrm{D}$ ultrathin nanomaterials possess a single or few layers, they are thought to be a perfect catalyst in many important electrochemical catalytic reactions. Since the thickness of the material is really less, it significantly improves its efficiency when analyzed against its counterpart bulk materials. Hydrogen evolution reaction (HER), oxygen evolution reaction (OER), oxygen reduction reaction (ORR), carbon dioxide reduction reaction $\left(\mathrm{CO}_{2} \mathrm{RR}\right)$, and nitrogen reduction reaction (NRR) are some of the immediate reactions in which electrocatalysts with high catalytic activities and stability are seen to increase the catalytic efficiency [152].

3D nanomaterials are ideal for electrochemical energy conversion devices. In general, an electrocatalyst's kinetic studies are linked to the structural morphology of the active phase, whereas the structure also influences its typical characteristic properties. Reduction in the catalyst size causes more active sites to be engaged to the electrolyte and can take part in reactions having a higher surface-to-volume ratio, causing an increase in its mass activity. However, shrinking the component size may harm efficiency, thus increasing its resistivity to charge transfer [152].

4.2.7. Nanocatalysts in Heterogeneous Hydrogenation and Hydrogenolysis. The activity of the hydrogen atoms presents on the reactant, along with the rate of adsorption of reactants onto the surface, can be improved by using heterogeneous catalysts. The catalytic activity and the synergetic interaction of the heterogeneous catalyst can be strengthened by using highly reduced titanium oxide ( $\mathrm{TiO})$ metal oxide support. The vacancies present inside the sublattices of titanium and oxygen atom in $\mathrm{TiO}$ improve the titanium monoxide polymorph, making it an excellent catalyst support and reaction promoter. As a result, the vacancies present within the sublattices of the heterogeneous catalyst improve the density of the donor state and the rate of hydrogen and substrate adsorption. Therefore, the dissociation process of hydrogen atoms from the catalyst surface requires less activation energy [335].
Consider $\mathrm{Pd} / \mathrm{TiO}$ nanocatalyst used for heterogeneous hydrogenation process. Titanium dioxide metal oxide support alters the physical properties of the nanocatalyst, thus creating unique sites that affect the behavior of the catalyst. The number of oxygen vacancies in titanium dioxide can be improved by introducing other metal atoms in the catalyst through doping. Titanium monoxide ( $\mathrm{TiO})$, when compared to titanium dioxide, contains a more significant number of vacancies within the sublattices of oxygen and metal atoms. Being a nonstoichiometric interstitial compound, $\mathrm{TiO}$ consists of a large fraction of catanionic and anionic vacancies. The density fraction of the vacancies present in the catalyst varies according to the number of oxygen atoms inside the catalyst and heat treatment. As long as these vacancies are randomly distributed or in a disorganized manner, it forms a polymorphically disordered cubic phase. Contrarily, when these vacancies are present in an organized manner, it forms a low-temperature polymorph that can be utilized as support for palladium loadings [335]. In these structures, most of the palladium particles are impregnated onto the skin of titanium monoxide, whereas the rest of the palladium particles are covered by titanium monoxide thin shells. During the hydrogenation reaction, the ions of palladium atoms, $\mathrm{Pd}^{2+}$, are initially reduced into $\mathrm{Pd}(0)$ by hydrogen molecules and loaded onto titanium monoxide. As the reaction proceeds, the hydrogen molecule dissociates on the surface of the palladium atom creating active species of hydrogen. These active species diffuse onto the catalyst's surface to interact with the lattices in titanium monoxide, thus producing hydrogenated titanium monoxide with a disordered surface. During this hydrogenation process, the surface of titanium monoxide undergoes surface reconstruction. As a result, the titanium monoxide thin shells spill over the palladium nanoparticles, improving the interaction of the metal with its support [335]. In reactions like the Fischer-Tropsch process, olefine synthesis, heterogeneous nanocatalysts containing iron metal support find many applications. The iron particles get deposited over the surface of the catalyst support, thus improving the selective dechlorination and hydro-dechlorination of organochlorine compounds, etc. During the hydrogenation process, iron nanoparticles conduct the reaction in hydrogen molecules. However, these metal nanoparticles undertake complete hydrogenation of alkynes to the alkanes under unsupported conditions [336].

Bimetallic nanomaterials are utilized for selective oxidation, selective hydrogenation, coupling reactions, hydrochlorination, amidation, reductive amination, hydrogenolysis, etc. [337]. Two mechanisms have been figured out to understand how bimetallic nanomaterials should be studied. The first mechanism is to prioritize understanding the chemistry involved and establish effective methods for attaining a definite control over the formation process during which the individual atoms convert to bimetallic nanomaterial particles. The second mechanism is to study the kinetics, theoretical modeling to design more effective catalysts. Even though several vital influencing factors like composition and structure have been analyzed, specific scientific issues like why adding the second metal 
increase the catalytic activity, which morphological feature is the most advantageous for the properties of the bimetallic nanomaterials remain unanswered. Given that the usage of bimetallic nanomaterial in asymmetric organic synthesis is limited, there is a significant opportunity to develop a better understanding of these materials' fundamental properties and practical applications [337].

During hydrogenolysis reaction, the single bond present between carbon atoms (C-C) or a carbon atom and a heteroatom (primarily oxygen) is broken down by hydrogen. During this process, hydrogen atoms are simultaneously added to the reactant, causing molecular fragmentation. Bimetallic nickel-iron catalysts are produced by coincidently impregnating the catalyst carrier with active metal salt precursors. The calcination follows this step under a noble gas atmosphere, followed by hydrogen reduction. During the hydrogenolysis reaction, the bimetallic $\mathrm{Ni}-\mathrm{Fe}$ nanocatalyst enters an alloy phase. During the alloy phase, the rate of adsorption of the reactant on the catalyst's surface is optimized, thus strengthening the activity of the catalytic material. Within the bimetallic surface, those sites contain iron adsorb reactant species such as the hydroxyl group, whereas the nickel-containing sites activate the hydrogen atom. The catalytic activity is improved within the iron-nickel bimetallic system by enchaining the synergetic interaction between the metal support and iron particles [336]. During $\mathrm{C}=\mathrm{O}$ hydrogenation and $\mathrm{C}=\mathrm{O}$ hydrogenolysis, the iron atoms present in the catalyst suppress the decarbonylation catalytic activity of nickel atoms. As the reaction proceeds, the synergetic metal-support interaction is improved by the bimetallic characteristic of the catalyst.

The nanocatalyst's electronic character and its microstructure affect the metal-to-metal or metal-to-carrier transfer of electrons. In $\mathrm{Ni}-\mathrm{Fe}$ bimetallic catalyst, the electronic density within the $\mathrm{d}$ orbitals of the iron atom varies according to the transfer of electrons from an iron atom to a noble metal with higher electronegativity. The rate of electron transfer can be improved by using an inert carrier like $\mathrm{SiO}_{2}$, thus improving the movement of metal precursors. As a result, reduction takes place, forming a metallic alloy. Additionally, within the bimetallic catalyst, the binding energies shift between the two metals, causing a transfer of electrons from iron to palladium atom. As the interaction between the metal atom increases, the reactivity increases, thus affecting the reaction route and the distribution of reaction products [336]. The magnetic properties of the metal carriers influenced the activity of the reaction and the products formed during the reaction, resulting in the rapid removal of the catalytic particles from the reaction medium. Moreover, it was observed that the amount of platinum loading influenced the hydrogenation reaction. Lesser the amount of platinum particles in spatial separation of the active sites during the dissociation of the hydrogen molecule, the lesser the contact between nitroarenes and platinum. This helps in preventing undesirable hydrogenation functional groups present in the reactant [336].
4.2.8. Advanced Oxidation Water Treatment Using Catalytic Nanomaterials. In most of the major metropolitan cities and towns, the drinking water undergoes a centralized treatment and is transported over a sizeable pipe-based network. But some challenges come with this method, some of them being limited resiliency against factors like climate change and population growth, as well as unsuitability in deserted communities. Therefore, a small-sized decentralized scheme is set up near the water-consuming communities to minimize the cost and energy demands. Moreover, advanced oxidation processes come in handy during unpleasant situations like these. Advanced oxidation processes treat drinking water as a polishing source to eliminate permeable pollutants, which can pass through the membrane, which are not treated by traditional processes otherwise. To address the fundamental and functional challenges posed by it, engineered nanomaterials (ENMs) are analyzed as heterogeneous catalysts because of their novel optical properties and electrical attributes, along with their suitability to fit within compact modular systems [338].

Advanced oxidation processes date back to the 1890s when the Fenton process was first introduced, which was a homogenous reaction involving the activation of $\mathrm{H}_{2} \mathrm{O}_{2}$, but it had a couple of problems, like the range of $\mathrm{pH}$ and the accumulation of iron sludge after neutralization. The heterogeneous Fenton process was thus developed to solve the limitations posed by the homogenous Fenton process. Efforts were then taken to utilize a weaker oxidant which led to using different species such as ozone and persulphates. Ozone was found to be quite an excellent oxidant for destroying pollutant particles because of its selective nature for electron-rich groups. Persulphates such as $\mathrm{HSO}_{5}$ (peroxymonosulphate) were found to be inexpensive as compared to their counterparts $\mathrm{H}_{2} \mathrm{O}_{2}$ as well as ozone. Despite the appeal of using the abovementioned processes, engineered nanomaterials (ENMs) based heterogeneous catalyst is a cost-effective alternative to homogenous municipal water treatment [338]. Considering Figure 23, photocatalytic advanced oxidation processes system containing reactor with suspended photocatalysts, UV-lamp (light source) for $\mathrm{TiO}_{2}$, and membrane filter that recovers the used catalyst. The photocatalytic semiconductor is excited by the photons, creating electrons in the conduction band and holes in the valence band. This is followed by a redox reaction at the catalyst surface using electron acceptors and electron donors.

The light-driven process involves photoexcitation of the semiconductor nanomaterial with the help of radiation from a lamp or sunlight to form a hole in the valence band and an electron in the conduction band. As a result, continuous administration of redox agents is no longer needed, making photocatalysis a novel technological application and an area of research on engineered nanomaterial in the field of environmental studies. Additional considerations, including budget and effects of life-cycle, are to be weighed in both of these advances in order to determine the true benefit of catalytic activity enhancement. Electrochemical advanced oxidation processes (EAOPs) pave the path from energy input 


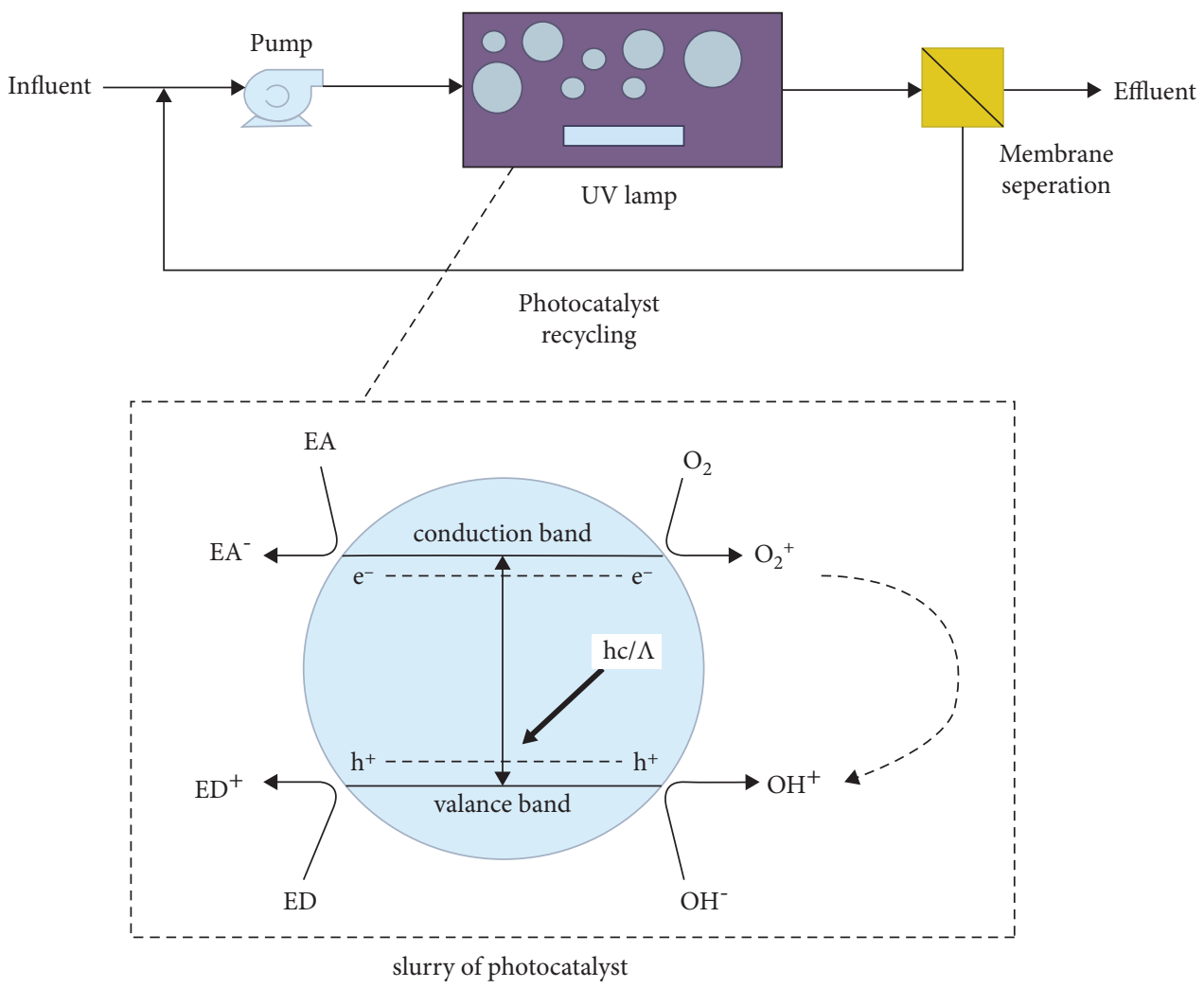

FIGURE 23: Photocatalytic advanced oxidation process system containing a reactor with suspended photocatalysts, UV-lamp (light source) for $\mathrm{TiO}_{2}$, and membrane filter that recovers the used catalyst. Diagram reproduced from Hodges et al. [338]. Challenges and prospects of advanced oxidation water treatment processes using catalytic nanomaterials. Nature Nanotechnology [338].

to contaminant oxidation. The process is carried out by applying a potential difference between the anode and cathode located inside a reactor, contained within the electrolyte, influent water. Organic compounds can be oxidized directly at the electrode surface via charge transfer or indirectly via reaction with electrochemically generated oxidants [338].

Some hurdles exist in the advanced oxidation processes. During the course of municipal water treatment, natural organic matter such as humic substances found from the decomposition of plant-based substances is found to be larger than anticipated, thereby reducing the effectiveness of this process due to radical scavenging and unnecessary adsorption onto the ENM surfaces. Another challenge that usually seems to be overlooked but might affect the industry largely is the presence of alkalinity and turbidity. Although rapid-mixed slurry systems were observed to have an efficient contaminant-to-surface mass transfer, the loss of engineered nanomaterials to the treated effluent stream may affect the environment. Membrane filtration is frequently used to separate engineered nanomaterial catalysts, which can shoot up the reaction cost and reaction complexity. Albeit gravity separation and magnetic separation strategies have been analyzed, their practical feasibility remains doubtful [338].

4.2.9. Transition Metal Catalyzed C-C Bond Formation. Recent years have got the researchers to extensively explore the world of transition metal-catalyzed carbon-carbon bond formation and carbon-nitrogen bond formation via carbonhydrogen activation and oxidative amination. Palladiumcatalyzed carbon-hydrogen activation reactions have emerged to be an effective method for synthesizing carboncarbon and carbon-nitrogen bonds since they provide an environment-friendly and more atom-efficient method, thereby reducing waste and improving economic feasibility. In this type of activation coupling and oxidative amination reactions, heterogeneous catalysts such as supported palladium nanoparticles would be a significant step toward going green. Moreover, they can also be recycled multiple times, making them more useful. A heterogeneous palladiumbased catalyst for the formation of carbon-carbon single bond, activation of carbon-hydrogen bond, and the formation of carbon-nitrogen bond via oxidative amination using $\mathrm{O}_{2}$ as the terminal oxidant under relatively mild conditions was formulated. Different temperature and pressure conditions were also checked thoroughly. Nanomaterials were found to be effective mainly because they are simple and no additional reagents are required [339].

4.2.10. Metal Nanoframes for Plasmonic and Catalytic Reaction. Nanoframes are those nanocrystals that have ridges without side faces. Generally, the synthesis of metal nanoframes is divided into two stages. The first stage of the process includes nucleation of the solid particles, followed by their growth. The interiors and side faces are hollowed out in 
the second stage, which can be accomplished through galvanic replacement reactions or chemical etching. But at the same time, etching can pose quite a few challenges. During corrosion, if the process is weak, it can lead to an incomplete hollowing, creating a partially open structure. If the corrosion is too strong, nanoframes may be over corroded and break apart into separate pieces. Nanoframes have high chemical stability, larger specific surface area, high thermal stability, and unorthodox surface atomic structures. All of the aforementioned reasons make metal nanoframes far better than their nanoparticulate counterparts. This is also why they have gained significant attention for catalytic applications. Additionally, since these nano frames have distinct and adaptable LSPR (localized surface plasmon resonances) properties and a high sensitivity factor, they have superior potential in optical sensing [340].

Five significant reactions have been discussed to synthesize metal nanoframes based on the mechanisms applied. The first mechanism is Galvanic replacement reaction oxidation of the template, followed by its dissolution, and finally reduction of the metal ions. The atoms that are formed get deposited on the template surface. Gold $(\mathrm{Au})$ based nanoframes are synthesized via galvanic replacement reactions between silver nanocubes and $\mathrm{HAuCl}_{2}$. Etching is the second mechanism in which the less stable elements present in the nanostructure undergo selective dissolution with an ideal etchant. Some of the common etchants used are $\mathrm{Fe}\left(\mathrm{NO}_{3}\right)_{3}$, $\mathrm{NH}_{4} \mathrm{OH}$, and sometimes $\mathrm{O}_{2}$ [340]. Etchant plays a critical role in synthesizing nanoframes mainly because we have to make sure that the power of corrosion is neither fragile to achieve dealloying, nor strong, thereby completely wrecking the product into pieces. The third mechanism is the Kirkendall effect, which mainly works on filling vacancies. Kirkendall effect is analyzed based on a balance of the mass flow of a faster diffusing species and the opposing flow of vacancies. This mechanism is applied to several processes aiming toward synthesizing hollow and porous nanocrystals. The fourth method includes the usage of electrodeposition, wherein the substance is deposited on the substrate with the help of an electric current. Electrodeposition can be coupled with etching to increase the efficiency of the nanoframe produced. The fifth method applies template-assisted growth wherein the template is used as a model structure in which the desired materials can be added. After the material has been added, an additional reaction takes place to remove the template and to let the newly formed nanoframe have the same size and shape corresponding to the parent template [340].

\subsubsection{Catalyst Self-Reconstruction in Electrocatalysis.} Recent advances in in situ and operando characterization techniques have revealed that most catalysts reconstruct their structure caused by electro-derived redox processes at a given potential, which is more than often followed by an increment or decrement in the activity of the catalyst. These self-changes to the structural morphology of the material make it challenging to truly identify the catalytically activated reaction sites, hampering the analysis of the actual catalytic mechanism [341].
Precatalysts act as a precursor or prerequisite for the selfreconstruction processes to originate. The morphological features of precatalysts play a massive role in determining whether or not the self-reconstruction process will occur. Reaction conditions should also be examined to determine the efficiency of the development of the product. When compared between ex situ and in situ operations, it was deduced that in situ operations combined with operando techniques are ideal for obtaining in-depth information about the structure. Even though some novel techniques concerning identifying the variations of the oxidation states and coordination structure have been developed, it still has a decent margin of error. Since the error has not been reduced for a while now, extracting the structural information of the precatalysts to facilitate the understanding of self-reconstruction becomes quite challenging. The development of structural self-reconstructing electrocatalysts can not only be used for quintessential catalytic reduction reactions but can also be used for new purposes to understand traditional heterogeneous catalysts. More research is required toward developing an understanding of ideal reaction conditions, morphological and mechanistic studies to make the selfreconstructing electrocatalysts succeed [341].

4.2.12. Nanozymes and Applications. Nanostructures have been quite helpful for several applications, one of them being their active employment in the field of biotechnology, chemical assays, medical imaging, and therapeutics. Fascinatingly, some nanomaterials have properties that resemble enzymes under physiological conditions. Such enzymes are called nanozymes. Nanozymes can thus replace natural enzymes and act as a primary low-cost alternative to the latter. Nanomaterials having enzyme-like properties have been used in many areas, including tissue regeneration, disruption of biofilms, and treatment of tumors. Ceria-based nanomaterials were observed to replicate superoxide dismutase, which showcased anti-inflammatory activities. Similarly, an FDAapproved iron oxide nanoparticle, ferumoxytol, was used for the treatment of iron deficiency which prevented the growth of tumors in mice. Many such developments are taking place right now, which has paved the way for newly found applications for catalytically active inorganic materials, in which their property of multifunctionality is utilized to treat infections in the biofilm, to locally trigger medications, serve as enzyme replacements, generate targeted cancer imaging and therapy, or prevent oxidative stress [342]. Nanozymes can be engineered in various ways to change their physicochemical characteristics and increase the activity of the catalyst. There are several methods for synthesizing various types of nanozymes in different morphologies and compositions, doping, surface alteration, and functionalization to refine the mechanism of the catalyst, biological functions, and biocompatibility as we want. Nanozymes may have one or more catalytic activities. Moreover, multiple catalytic activities can be achieved by assembling different nanozymes into one unit. In addition to that, hybrid materials utilized for multifunctional devices can be formed by integrating them with natural enzymes [342]. 
Nanozymes possess high stability and are cost-efficient as opposed to natural enzymes, which pave the way into several applications and developments in the future. Given that these nanozymes have a small size, nanoparticles are absorbed by cells or penetrated into tissues by moving in between cells and using the process of diffusion through the extracellular matrix. One of the most well-studied applications of catalytic nanoparticles is analyte detection. Ammonia detection was possible by $\mathrm{LaCoO}_{3}$ nanoparticles, which catalytically enhanced the oxidation process where ammonia converts into $\mathrm{NO}_{x}$ molecules which, when reacted with luminol, can be detected by light emission. This process is used to detect several other compounds such as hydrogen peroxide as well as glucose. These methods are used to identify Ebola, tumor, cancer, and anti-biofilm, etc. It was found that nanoparticles bound to biofilms (bacterial clusters which adhere to a surface, making it difficult to remove) are catalytically active. These nanoparticles support the generation of free radicles from $\mathrm{H}_{2} \mathrm{O}_{2}$, which destroys the components and removes the bacteria in the biofilm. These nanoparticles were found to have great disease-preventing powers in both in vivo and in vitro [342].

Albeit there is a great potential for enzyme-like nanomaterials, some parameters act as hurdles and barriers, one of them being that the enzymatic properties and kinetics cannot be controlled. The change of surface chemistry can pose a challenge in the long run. The design of such nanomaterials must be carefully done to avoid the potential for cytotoxicity. Nonetheless, given the distinct characteristics of nanocatalysis-based technology, additional research may result in a unique multifunctional platform for the production of sustainable as well as economically feasible therapeutics for human use [342].

4.2.13. Biomimetic Catalysis. Chiral nanomaterial and nanoparticle-based biomimetic catalysts are refined enzymebased nanomaterials that essentially work by simulating naturally occurring enzymes by including various artificial nanomaterials that possess higher catalytic activity. These nanoparticle-based enzymes are better than conventional enzymes due to their lower cost, stability, easy handling and storage, purification, more catalytically active sites, and easier mass processing, resulting in their widespread use. For potential catalytic interaction to take place in the near future, recent research has revealed that the appropriate size, morphology, and precise exposure of biomimetic nanomaterials are needed. All of the methods used in biomimetic catalysis have great significance in improving the selectivity of the substrate. High output at the same time is also possible, courtesy of the assistance of external driving forces. These concepts play a pivotal role in finding structures that are versatile and relatively easy to tune, which in turn becomes easy to cope with the current complex biological environment [343].

Chiral nanomaterials have been in the limelight in terms of their efficiency and stability. They contain individual nanoparticles arranged into a larger structure via external and internal forces. Many chiral superstructures can be formed using the abovementioned mechanism of rational assembly of several different fundamental blocks of nanoparticles. The critical points for synthesizing chiral nanoparticles and nanoassemblies for biomimetic catalysis in this study and some principles for controlling their bioactivity that is either internal or external factors were demonstrated. Despite the fact that chiral nanomaterials can be precisely regulated, there are currently no specific artificial methods to replicate natural active sites for improving biological interactions. Nonetheless, it is essential to test new methodologies for improving the activity of chiral nanomaterial catalysts. Since single component nanomaterials are not that useful in the field of biocatalysis, biomimetic nanomaterials' multifunctionality is utilized for biogenic cascading reactions. Other than the typical applications, they can also be used for DNA cutting, cellulose degradation, immunological therapy, etc. Albeit the advantages of chiral nanomaterials, there still exists a certain scope of improvement that the researchers can exploit, and it is to be made sure that these challenges can be defeated in the years to come [343].

\subsubsection{Electrocatalysis Using Pyrite Type Nanomaterials.} Noble metal-based catalysts are emerging as a source of adoption for carrying out various electrode reactions, such as anodic oxidation of $\mathrm{H}_{2}$, cathodic reduction of $\mathrm{O}_{2}$ for fuel cells, and many more. However, whether the noble metals are being utilized, recent developments in the manufacturing of catalysts for fuel cells can be differentiated. The majority of emerging noble-metal catalyst strategies focus on adjusting the structure and composition of the surface to allow improved catalytic activity with less expensive noble metals. Even with the significant progress, partial replacement of noble metals does not fully resolve the high expenditure of money, given demand/price fluctuations. So, the advancement of nonnoble metal catalysts is prevalent right now, which has excellent properties, thereby phasing out the noble metal catalysts and reducing the costs. These catalysts include many nanomaterials being used in them, which made the researchers realize that nanostructured items could be of great use when it comes to advanced electrolysis reactions [344].

In particular, pyrite structured nanomaterials are very promising given that they could be made at the nanoscale, having various properties. Nanostructured pyrites are nowadays generating many possibilities in electrocatalysis. Production of pyrites with regulated sizes, parts, and modifications of structure should be done according to the requirement, which makes pyrites quite flexible. Material grafting can be one such process that significantly improves pyrite nanostructures' catalytic performances due to optimized electronic structures and combined catalytic proficiencies. Albeit the progress is clearly shown, we cannot deny that the research and development of said pyrites have recently begun and needs some time and hard work to come into the spotlight. A better form of development for having acute control over the pyrite structure, dimensions, and their component analysis are still desperately required. Materials grafting, electronic structures, active sites, defects, and 
doping, and how these affect catalytic properties are fundamental aspects of pyrite modification developments that are still poorly understood. Future experimental works may be able to shed light on the aforementioned issues. In the years to come, it is believed that pyrite-based nanostructures will take over the catalyst market and act as a cheaper and more reliable alternative to noble metal electrocatalysts [344].

\subsection{Nanocatalysts in Hydrocarbon Processing}

4.3.1. Carbon Nanotubes. CNTs are known to have higher stability and good physicochemical properties. The addition of $\mathrm{sp}^{3}$ bonds resulted in higher reactivity. CNTs have highly functionalized surface properties and homogeneous microstructures. However, it was found that CNTs decompose at temperatures higher than $550^{\circ} \mathrm{C}$, and hence, for catalytic cracking operations, they need to be shielded with metallic oxides for resistance. It was found that, by covering CNTs with $\mathrm{SiO}_{\mathrm{x}}$ tetraethoxysilane (TEOS), CNTs get better thermal resistance and can be used at higher temperatures. When the catalytic cracking of naphtha was carried out in a fixed bed reactor using modified CNTs (by adding silicon layers for better stability), ethylene and propylene's yield reached a maximum of only $26.5 \%$ wt. at $680^{\circ} \mathrm{C}$. However, when one gram of CNT was increased, the total yield of olefins increased to $36.31 \%$ wt. When the silicon coating was varied (from $5 \%$ to $15 \%$ by wt.), in another experiment, the total yields were $18 \%$ for ethylene and $25 \%$ for propylene. This is $280 \%$ higher than what is obtained as yield in thermal cracking without catalysts. CNTs with $10 \%$ Si showed to have the best yields compared to others [30].

One of the studies synthesized a novel mesoporous solid acid catalyst (HPMo-Ni/ CNT). Basically, Ni/phosphomolybdic acid is immobilized on carbon nanotubes and then used for the catalytic cracking of Jatropha oil. A significant amount of catalytic cracking activity was observed. The biodiesel yield reached about $86.7 \%$ wt., and the conversion reached about $98.2 \%$. The conversion was at $86.7 \%$ even after the fifth cycle of recycling. This reaction required the formation of $<\mathrm{C} 15$ alkanes. This was accelerated due to the CNT's favorable environment for diffusion and electroconductivity. Coke resistance was noticed in this catalyst, and it is attributed to the repulsive forces of the carbon atoms [4].

4.3.2. Mechanochemically Produced Nanocatalysts. Wang et al. also used a simple mechanochemical solid-state reaction with $\mathrm{SrCO}_{3}$ and $\mathrm{TiO}_{2}$ in a stoichiometric ratio at $1100^{\circ} \mathrm{C}$ to make fluorine-doped $\mathrm{SrTiO}_{3} 85$ particles. In the following phase, different fluorine sources such as polytetrafluoroethylene (PTFE), $\mathrm{SrF}_{2}$, and $\mathrm{LiF}$ were utilized to obtain F-doped materials [229].

${ }^{90}$ Fluorine doping caused a redshift in $\mathrm{SrTiO}_{3}$ 's absorption edge, narrowing the bandgap. Under visible light $(>400 \mathrm{~nm})$ and near-ultraviolet light $(>290 \mathrm{~nm})$ irradiation, the photocatalytic activity of $\mathrm{SrTiO}_{3}$ and F-doped $\mathrm{SrTiO}_{3}$ in the oxidative breakdown of $\mathrm{NO}$ was investigated. When compared to undoped $\mathrm{SrTiO}_{3}$, F-doped $\mathrm{SrTiO}_{3}$ had three times the activity. $\mathrm{TiO}_{2}$ can be organically functionalized via a reactive ball milling approach in addition to doping. Using a high-energy planetary ball mill, a new approach for manufacturing phenyl phosphonic acid-functionalized titania particles has recently been developed. This technology could provide a simple and advanced way for producing inorganic NPs and surface functionalizing them in situ for various catalytic applications. The most often utilized photocatalysts are $\mathrm{ZnO}$-type compounds. The photocatalytic destruction of resorcinol in water was demonstrated using $\mathrm{ZnO}$ catalysts produced by grinding zinc oxide and oxalic acid in an agate mill followed by thermal decomposition. Because of agglomeration, a higher calcination temperature increased the crystallite size, lowering the photocatalytic activity [229].

In one of the studies, a simple one-step pyrolysis technique was used to prepare $\mathrm{Ni} / \mathrm{Fe}$ nanofibers/porous carbon catalyst for high-grade $\mathrm{H}_{2}$-rich syngas generation from pyrolysis-gasification of biomass and plastic wastes. The bimetallic Ni/Fe CNF/PCs catalyst produced the best yield and had better selectivity of $\mathrm{H}_{2}$ and $\mathrm{CO}$ when compared to monometallic NiCNF/PCs and FeCNF/PCs catalysts. With the outstanding dispersibility of the $\mathrm{Fe}_{0.64} \mathrm{Ni}_{0.36}$ alloy nanoparticles and the nanofibers/porous carbon composite structure, the bimetallic $\mathrm{Ni} / \mathrm{Fe} \mathrm{CNF} / \mathrm{PCs}$ catalyst appeared to be the best balance between catalytic activity and stability, according to the cycle stability test. Bimetallic $\mathrm{Au} /$ $\mathrm{Ag}$ and trimetallic Pd-Au/Ag core-shelled NPs with welldefined bimetallic $\mathrm{Au} / \mathrm{Ag}$ and $\mathrm{Pd}-\mathrm{Au} / \mathrm{Ag}$ core-shelled NPs were recently produced and applied to the electro-oxidation of glycerol. Surprisingly, both $\mathrm{Au}-\mathrm{Ag}$ and $\mathrm{Pd}-\mathrm{Au} / \mathrm{Ag} \mathrm{NPs}$ are active in alkaline solutions but not in acidic solutions. The $\mathrm{Pd}-\mathrm{Au} / \mathrm{Ag}$ catalyst has higher selectivity than Au-Ag NPs, yielding dihydroxyacetone 70.1 percent of the time. The Pd$\mathrm{Au} / \mathrm{Ag}$ core-shell catalyst prevents the cleavage of CC bonds, allowing dihydroxyacetone to develop [229].

4.3.3. Core-Shell Nanomaterials. Bimetallic gold-silver coreshell NPs catalyzed biodiesel production, resulting in $86.9 \%$ yield. Core-shell NP catalyst such as Ag encapsulated by Pd shell and supported by $\mathrm{ZnO}$ nanorods enhances the selectivity of hydrogenation of $\mathrm{CO}_{2}$ to methanol over the production of CO. A Pd centered core-shell catalyst, made by encapsulating Pd NPs inside silicate zeolite crystals ( $\mathrm{Pd} / \mathrm{Sil})$, has unusual activity in the hydrogenation of biomass-derived furfural, with selectivity to furan of up to $98.7 \%$. After the reaction, the catalyst can be easily regenerated by calcination at $550.8^{\circ} \mathrm{C}$. The regenerated $\mathrm{Pd} /$ Sil catalyst activity is comparable to that of the virgin catalyst, and no aggregation or degradation was detected. The core-shell catalyst has the advantage of combining very stable Pd NPs with active sites and zeolite micropores, which controls reactant and product diffusion to and from the active Pd core [230].

4.3.4. Layered Double Hydroxides (LDH). Xinjiang and coworkers prepared a Ni/ $\mathrm{Al}_{2} \mathrm{O}_{3}$ core-shell catalyst by the calcination of supported $\mathrm{Ni}$-Al layered double hydroxides 
on the surface of activated carbon. The metallic Ni core forms with an amorphous aluminum oxide shell formed by the AlIII species in the $\mathrm{LDH}$ during calcination. The synthetic method prevents Ni/NPs from aggregating, resulting in highly scattered Ni/NPs with $14-15 \mathrm{~nm}$ diameters. In the reductive catalytic fractionation of lignin to phenolic monomers, the $\mathrm{Ni} / \mathrm{Al}_{2} \mathrm{O}_{3}$ core-shell catalyst demonstrated good catalytic activity. At $190.8^{\circ} \mathrm{C}$, oak wood, for example, is converted to phenolic monomers at a 23.4 percent conversion rate. Indeed, the yield of phenolic monomers obtained with the $\mathrm{Ni} / \mathrm{Al}_{2} \mathrm{O}_{3}$ core-shell catalyst, such as 4-n-propyl-guaiacol, 4-n-propenyl-guaiacol, 4-n-propyl-syringol, and 4-n-propenyl-syringol, is substantially higher than that obtained with $\mathrm{Ni}$ immobilized on activated carbon without a core-shell structure. Note that, in reductive catalytic fractionation, solvolysis breaks down the lignin structure into small fragments, sometimes referred to as lignin oil. The catalyst is responsible for hydrogenolysis. The hydrogenation of these fragments is small enough to pass through the mesoporous catalyst shell [231].

4.3.5. Gamma- $\mathrm{Al}_{2} \mathrm{O}_{3}$ Doped with $\mathrm{MnO}_{2}$. The $\mathrm{SO}_{2}$ absorption activities by blank $\gamma-\mathrm{Al}_{2} \mathrm{O}_{3}$ and different metal loaded catalyst-sorbents were evaluated and shown by Wang et al. The $\mathrm{SO}_{2}$ concentration curve via Blank $/ \mathrm{Al}_{2} \mathrm{O}_{3}$ shows the physical adsorption of $\mathrm{SO}_{2}$ on the surface of $\mathrm{Al}_{2} \mathrm{O}_{3}$. The breakthrough time is substantially shorter than the others, at only 21 minutes, and the outlet $\mathrm{SO}_{2}$ continues to rise after that. The sorbents all perform better with metal oxides loaded than with blank $/ \mathrm{Al}_{2} \mathrm{O}_{3}$, indicating that the selected metal oxides have some catalytic impact in the $\mathrm{O}_{3}$ enriched atmosphere [345].

Overall, $\mathrm{MnO}_{2} / \mathrm{Al}_{2} \mathrm{O}_{3}$ has the best $\mathrm{SO}_{2}$ adsorption capability, with a breakthrough time of 67 minutes, three times that of pure- $\mathrm{Al}_{2} \mathrm{O}_{3}$. The explanation for this can be linked to the more efficient breakdown of $\mathrm{O}_{3}$ and the generation of highly active atomic oxygen on the catalyst surface with the help of $\mathrm{MnO}_{2}$. The breakthrough time for $\mathrm{Cr}_{2} \mathrm{O}_{3} / \mathrm{Al}_{2} \mathrm{O}_{3}$ is roughly 8 minutes, even faster than pure $\mathrm{Al}_{2} \mathrm{O}_{3}$. However, the rate of increase in outlet $\mathrm{SO}_{2}$ following breakthrough is slow enough. Some factors (preparation factors and operating conditions) which could have affected the catalysts are (1) calcination temperature, (2) $\mathrm{MnO}_{2}$ loaded, (3) reaction temperature, and (4) NO in the flue gas [345].

Calcination temperature is one of the most critical elements affecting catalyst activity, as it has a significant impact on the final states of loaded metal crystals on the surface of catalyst sorbents and catalyst characteristics. The breakthrough time is reduced from 80 minutes to 67 minutes and 66 minutes, when the calcination temperature is increased from $573 \mathrm{~K}$ to $673 \mathrm{~K}$ and $773 \mathrm{~K}$. Similarly, the $\mathrm{SO}_{2}$ removal efficiency has decreased from 93.7 percent to 90.3 percent and 88.9 percent, respectively. One of the critical reasons for catalyst deactivation will be a slight decrease in pore volume and surface area induced by the high-temperature calcination procedure. The aggregation of loaded metals caused by partial melting will also significantly damage the active sites on the catalyst-sorbent surface [345].

However, at high temperatures, such as $773 \mathrm{~K}(57.7 \%)$, the $\mathrm{SO}_{3} / \mathrm{SO}_{x}$ ratio was larger than at $673 \mathrm{~K}(45.9 \%)$ and $573 \mathrm{~K}$ (44.9\%). The mass fraction of $\mathrm{Mn}$ was utilized to calculate the amount of $\mathrm{MnO}_{2}$ loaded. Clearly, the number of loaded metals affects the performance of the catalyst sorbents. The breakthrough time is around 71 minutes with 4 percent Mn loaded, and the $\mathrm{SO}_{2}$ removal efficiency is $87.8 \%$ at 120 minutes. The breakthrough time increases to 80 minutes for the $8 \%$ condition, resulting in a 93.8 percent $\mathrm{SO}_{2}$ removal efficiency. When the loaded amount is increased to 12 percent, the breakthrough time and efficiency drop to 61 minutes and 85.4 percent, respectively; by increasing the amount of $\mathrm{Mn}$ loaded in the loaded metal monolayer, the dispersion of the loaded metal monolayer can be enhanced. However, aggregation of active metals and blockage of some porous structures may cause catalyst-sorbents with large amounts of metals to deactivate. As a result, the loaded metal oxides will have an optimal amount. For $\mathrm{MnO}_{2}$, an amount of 8 percent for the catalyst-sorbents should suffice. The results show that the ability of $\mathrm{MnO}_{2} / \mathrm{Al}_{2} \mathrm{O}_{3}$ to collect $\mathrm{SO}_{2}$ has a nonlinear relationship with reaction temperature. The breakthrough time increases from $30 \mathrm{~min}-$ utes to 40 minutes as the reaction temperature rises from $373 \mathrm{~K}$ to $393 \mathrm{~K}$, and the $\mathrm{SO}_{2}$ removal efficiency rises from 80 percent to 80.6 percent. Increasing the reaction temperature to $413 \mathrm{~K}$, the breakthrough time is extended to 80 minutes, more than double the period at $393 \mathrm{~K}$. The $\mathrm{SO}_{2}$ removal efficiency increases to 93.8 percent. The flue gas temperature behind the air preheater is always below $423 \mathrm{~K}$ in a conventional coal-fired power station to improve thermal efficiency [346].

In most cases, the combustion exhaust contains a considerable amount of $\mathrm{NO}_{x}$ in addition to $\mathrm{SO}_{2}$. Also, in the simultaneous removal of $\mathrm{NO}_{x}$ and $\mathrm{SO}_{2}, \mathrm{NO}_{x}$ will invariably coexist with $\mathrm{SO}_{2}$ during the oxidation process. Because $\mathrm{NO}$ is the most common $\mathrm{NO}_{x}$ component in combustion flue gas, the influence of $\mathrm{NO}$ on the oxidation of $\mathrm{SO}_{2}$ was studied here. We employed a $2 \mathrm{~g} \mathrm{MnO} \mathrm{O}_{2} / \mathrm{Al}_{2} \mathrm{O}_{3}$ catalyst sorbent loaded with $8 \% \mathrm{Mn}$ and calcined at $573 \mathrm{~K}$. The effect of $\mathrm{NO}$ on the removal of $\mathrm{SO}_{2}$ was xcompared with the amount of ozone delivered to the reaction system, according to the stoichiometric ratios $\left[\mathrm{O}_{3}\right] /\left[\mathrm{SO}_{2}\right]=1$ and $\left[\mathrm{O}_{3}\right] /\left[\mathrm{SO}_{2}+\mathrm{NO}\right]=1$ for the circumstances without $\mathrm{NO}$ and with $\mathrm{NO}$ in the flue gas [347]. With NO's presence in the reaction system, the breakthrough time increases from 80 to 100 minutes. Additionally, during 120 minutes, the $\mathrm{SO}_{2}$ removal effectiveness increases from 93.8 percent to 96.7 percent. NO in the flue gas can be easily converted to $\mathrm{NO}_{2}$ via homogeneous reactions with $\mathrm{O}_{3}$ in the presence of $\mathrm{NO}$ and $\mathrm{SO}_{2}$, as previously investigated. The presence of $\mathrm{NO}_{2}$ can further oxidize $\mathrm{SO}_{2}$ to produce $\mathrm{SO}_{3}$, which was formerly used to produce sulfuric acid ( $\mathrm{Li}$ et al., 2002). There will be the following reactions:

$$
\begin{aligned}
& \mathrm{NO}+\mathrm{O}_{3} \longrightarrow \mathrm{NO}_{2}+\mathrm{O}_{2} \\
& \mathrm{SO}_{2}+\mathrm{NO}_{2} \longrightarrow \mathrm{SO}_{3}+\mathrm{NO}
\end{aligned}
$$

4.3.6. Highly-Dispersed $\mathrm{Cr}_{2} \mathrm{O}_{3}-\mathrm{ZrO}_{2}$ Binary Oxide Nanomaterials. $\mathrm{Cr}_{2} \mathrm{O}_{3}-\mathrm{ZrO}_{2}$ binary oxide catalyst was 
prepared using a coprecipitation method, and different variants were produced ( $\mathrm{Zr}$ composition at $0 \%, 25 \%, 50 \%$, $75 \%$, and $100 \%)$. The catalyst's reusability was also studied by regenerating it after the experiment. Ethylene and diethyl ether were formed in the range of $200-400^{\circ} \mathrm{C}$ via dehydration. In the meanwhile, acetaldehyde was formed via dehydrogenation. The conversion was the highest for the catalyst having Zr 75\% (CZ75) throughout various reaction temperatures. The next best variant was CZ25. Even in the yield \% of ethylene, CZ75 had the highest at about more than $70 \%$. CZ100 had the next best yield \% for ethylene. However, for acetaldehyde production, the selectivity was CZ0 $>$ CZ25 $>$ CZ50 $>$ CZ75 $>$ CZ100. This shows contrasting trends over different processes. These things show a high dependence on the $\mathrm{Cr} / \mathrm{Zr}$ proportion by the catalyst. The highest specific rate was also observed for CZ75, which showed homogeneous pore distribution. CZ75 also had one of the best reusability features and percentages compared to the other variants [232].

The 2,3-position of geraniol is preferentially epoxidized, generating 2,3-epoxygeraniol as the primary product. The $\mathrm{PW} 11 \mathrm{Fe} / \mathrm{SiO}_{2}$ nanocatalytic system provided the highest conversion percentage and selectivity among the several polyoxotungstates used. Pereira et al. immobilized oxidovanadium IV acetylacetonate $\left[\mathrm{VO}(\mathrm{acac})_{2}\right]$ onto silica NPs and then functionalized it with 3-aminopropyltriethoxysilane to develop superior inorganic-organic hybrid nanocatalysts that combine high substrate conversion and selectivity with low reaction time. They then investigated its catalytic application in the allylic epoxidation of geraniol. The nanocatalyst had a conversion rate of $100 \%$ and a selectivity of $99 \%$ for the 2,3-epoxygeraniol product, and it was stable after four cycles of use. Liu et al. have investigated the prospect of employing gold NPs (GNPs) as suitable transition-metal catalysts in a one-pot synthesis of a gold-silica nanosphere catalyst for styrene epoxidation. 58 silica nanospheres with symmetrical mesopores and distributed silanol groups may be able to ensure excellent GNP dispersion. The performance of the Au-silica catalyst (epoxidation selectivity in styrene oxidation) was compared to that of previously reported gold-based catalysts for the procedure.

The $\mathrm{Au} / \mathrm{SiO}_{2}$ catalyst outperformed metal-oxide-supported $\mathrm{Au} / \mathrm{MgO}$ and $\mathrm{Au} / \mathrm{Al}_{2} \mathrm{O}_{3}$ catalysts in terms of styrene conversion and epoxidation selectivity.

4.3.7. $\mathrm{TiO}_{2}$ Nanoparticles. The $\mathrm{TiO}_{2} \mathrm{NFs}$ showed the highest conversion $\%$ of about $66 \%$ for the cyclohexane and also the highest selectivity on average. Others showed $58 \%$, while $\mathrm{TiO}_{2}$ NTs showed only $37 \%$. Under the test circumstances, no hydroperoxide products were found. Due to the conformational nature of the substrate, there is no evidence for a link between the number of acidic sites on the catalyst and the product's selectivity in this situation. $\mathrm{TiO}_{2} \mathrm{NFs}$ also showed about $71 \%$ conversion in the oxidation of indane, which was the highest compared to others. It also showed about $82 \%$ selectivity for the desired product in the oxidation of indane. In the allylic oxidation of toluene using molecular oxygen, the $\mathrm{TiO}_{2}$ nanocatalysts did not demonstrate considerable activity, consistent with prior findings. However, when using $\mathrm{TiO}_{2}$ NPS as the catalyst and TBHP (30 mol\%) as an initiator, 34 percent conversion was achieved with total selectivity for benzaldehyde. This discrepancy in allylic oxidation between indane and toluene could be due to a secondary benzylic carbon in indane, which oxidizes more easily in radical chain reactions than toluene's primary benzylic carbon. When compared to the other catalysts examined, $\mathrm{TiO}_{2}$ NFs performed somewhat better as promoters for oxidation reactions. $\mathrm{TiO}_{2} \mathrm{NFs}$ also showed better catalyst recyclability (running for 5 cycles) [235].

As a result, it appears that activities are not only dependent on the catalysts' precise surface area. The type of crystalline structure and surface shape discovered in each catalyst can potentially explain the reported catalytic activity. At ambient temperatures, rutile is recognized to be the most stable form of $\mathrm{TiO}_{2}$. Previously, atomistic simulations of $\mathrm{TiO}_{2}$ polymorph surface structures revealed that the rutile phase had more exposed faces with high energy than the anatase phase. Furthermore, the exposed rutile faces $\left(\begin{array}{lll}0 & 1 & 1\end{array}\right)$ and $\left(\begin{array}{lll}1 & 1 & 1\end{array}\right)$ were identified as highly active oxidation surface faces. Due to the dominance of the rutile phase in $\mathrm{TiO}_{2} \mathrm{NFs}$ in our research, this catalyst has exhibited higher activity for oxidation processes than other nanomaterials. The cyclohexene oxidation process with P25 containing pure rutile (verified by XRD) yielded an 86 percent conversion rate with a $90 \%$ selectivity.

4.3.8. Esterification Reaction. The conversion rates of alcohols were found to be near to $100 \%$, indicating that just a tiny amount of catalyst was required to catalyze the process, especially when compared to the previously studied catalytic systems. Zhang and coworkers recently published another remarkable example of a solid-supported catalyst. They devised a cost-effective method for producing hybrid hollow nanospheres (HNs) with many sulfonic groups uniformly dispersed in mesoporous silica channels. The sulfonation of silica HNs with polystyrene (PS), which was highly dispersed in the mesoporous shell and had been prepared by cocondensing a mixture of TEOS and alkoxysilyl-functionalized poly (methyl acrylate) (PMA) around PS nanospheres in a basic medium using CTAB as a structural directing agent followed by the addition of THF was involved in the fabrication procedure. In acid-catalyzed liquid processes, such as the esterification of lauric acid with ethanol, the hybrid HNs could be effective solid acid catalysts [233].

4.3.9. In Situ Catalytic Upgrading. For in situ heavy oil upgrading, Hart and coworkers compared bionanoparticles ( $\mathrm{Pd} /$ biomass) to many different catalysts, including $\mathrm{Pd} /$ $\mathrm{Al}_{2} \mathrm{O}_{3}, \mathrm{Pd} / \mathrm{C}, \mathrm{Al}_{2} \mathrm{O}_{3}$, and Co-Mo/ $/ \mathrm{Al}_{2} \mathrm{O}_{3}$. The Pd/biomass catalyst increased the produced oil gravity by 7.8 API while lowering the viscosity to $7 \mathrm{mPa}$.s. Pd/biomass catalyst also had a lower coke yield (3.87 wt.\%) than $\mathrm{Al}_{2} \mathrm{O}_{3}$ (13.65 wt.\%), $\mathrm{Pd} / \mathrm{Al}_{2} \mathrm{O}_{3}$ (9.55 wt.\%), and $\mathrm{Pd} / \mathrm{C}$ (9.55 wt.\%) catalysts (6.85 wt. percent). Furthermore, as compared to conventional 
thermal cracking and upgrading utilizing other catalysts, the $\mathrm{Pd} /$ biomass catalyst demonstrated a considerable reduction in coke production, which could greatly improve deactivation resistance in the field [234].

The use of Ni-Co modified g-alumina $\left(\mathrm{NiCo} / \mathrm{g}-\mathrm{Al}_{2} \mathrm{O}_{3}\right)$ as a catalyst for heavy oil upgrading was described by Avbenake et al. The API gravity, hydrogen/carbon ratio, and light oil yields were somewhat more significant for the reaction under nitrogen atmosphere without a liner than for the reaction under a hydrogen atmosphere with liner, according to the results of the catalytic performance test.

Despite this, the catalytic reaction under nitrogen produced $0.2 \mathrm{wt}$. percent coke, whereas hydrogen produced no coke. The viscosity and sulfur concentration of the former reaction was lower than those of the latter reaction. In the toe-to-head air injection (THAI) process, a pelletized Ni$\mathrm{Mo} / \mathrm{Al}_{2} \mathrm{O}_{3}$ catalyst was also introduced for in situ catalytic upgrading of heavy oil and bitumen. The improved oil had a viscosity reduction of up to 5 times that of the feed oil, according to the catalytic activity evaluation (0.49 Pa.s).

The API gravity of the feed oil increased by about 2 to 5 , while the gasoline output increased by 2.5 to 13 weight percent. Sulfur and metals ( $\mathrm{Ni}$ and $\mathrm{V}$ ) levels were reduced by $2-8 \%$ and $1.3-9.2$ percent, respectively [234].

4.3.10. Catalytic Cracking. Khalil and coworkers recently reported on the successful use of nontoxic and low-cost hematite nanoparticles for heavy oil aquathermolysis. Using thiophene as a molecular model, the performance and catalytic mechanism of the nanocatalyst were investigated in the presence and absence of tetralin as a hydrogen donor. According to the findings, the conversion of thiophene to maleic acid, $\mathrm{SO}_{2}$, and $\mathrm{CO}_{2}$ were caused by oxidative desulfurization of thiophene at the surface of hematite nanoparticles. The catalytic activity of the nanocatalyst was also found to increase with the length of the aquathermolysis reaction time and temperature, as well as the number of catalysts used. Nonetheless, the inclusion of tetralin as a hydrogen donor was found to be responsible for a modest decrease in hematite nanoparticles' catalytic activity. According to the paper, such a phenomenon is most likely due to hydrogen donors' ability to occupy certain portions of the catalyst surface and obstruct the active catalytic site. The potential of hematite nanoparticles to facilitate the aquathermolysis reaction of heavy crude oil was further elaborated in another work by surface modification with oleic acid. Due to the change in particle hydrophobicity from hydrophilic to slightly hydrophobic, the performance of surface-modified hematite nanoparticles was shown to be superior to that of untreated ones. Surface-modified hematite nanoparticles were able to reduce the viscosity of heavy oil by 74.33 percent in such a mild reaction situation, according to the findings.

A comparison of macro-ZSM-5 (2 mm) and nano-ZSM-5 $(100 \mathrm{~nm})$ in the catalytic cracking of naphtha was carried out by Konno et al. The nano-ZSM- 5 was more effective $(h=0.99)$ than the macro-ZSM-5 $(h=0.21)$, indicating that the nano-ZSM-5 had more accessible sites.
As a result, the nano-ZSM-5's reaction rate constant was 4.7 times higher than the macro-ZSM-5's. Furthermore, the stability test revealed that the activity of macro-ZSM- 5 decreased with time on stream, whereas nano-ZSM-5's high activity could be maintained for up to 250 hours. Taufiqurrahmi et al. used a diluted and viscous gel to prepare nanosized zeolite $\mathrm{Y}$ and beta. Tetramethylammonium (TMA) ion directs the former, whereas tetraethylammonium (TEA) ion aids in the creation of the latter. Both catalysts demonstrated higher conversion and selectivity toward the gasoline fraction in the catalytic cracking of palm oil than their bigger counterparts. These results were attributed to the nanosized zeolites' increased microporosity and active sites.

Wakihara and coworkers described a top-down method for the synthesis of nanosized ZSM-5, as opposed to the more conventional bottom-up method. They used a bead milling process on raw ZSM-5 with an average particle size of $3 \mathrm{~mm}$, which resulted in a considerable particle size reduction of around $100 \mathrm{~nm}$. Because of the high mechanical force used during bead milling, this smaller particle had a low crystallinity, with a crystalline center and amorphous outside half. After bead milling, the products were recrystallized in an alkaline solution using the hydrothermal technique. The crystalline core served as nuclei in this example, whereas the amorphous sections were eaten by crystal development. As a result, well-defined nanosized ZSM-5 with sizes ranging from 30 to $100 \mathrm{~nm}$ were produced. After the tenth pulse number, the nanosized ZSM-5 exhibited a consistent benzene production $(>90 \%)$, whereas the raw ZSM-5 had a lower benzene yield (70\%) with a significant fall at the third pulse number. Katja and coworkers have described a top-down strategy that is comparable to theirs. They used a surfactant, cetyltrimethylammonium bromide, to perform successive planetary ball milling and recrystallization (CTAB). Surfactants control crystal development and aggregation, resulting in nanosized ZSM-5 crystals. The nanosized ZSM-5 is very active in LDPE catalytic cracking, with observed activation energy (Jobs) of $132 \mathrm{~kJ} \cdot \mathrm{mol}^{-1}$ which is lower than the Jobs of raw ZSM-5 catalyzed LDPE cracking $\left(224 \mathrm{~kJ} \cdot \mathrm{mol}^{-1}\right)$ [234].

4.3.11. Hydroprocessing of Crude Oil. The catalytic performance of supported $\mathrm{Ni}_{2} \mathrm{P}$ and $\mathrm{Pt}$ catalysis for the hydroprocessing of vegetable oils into normal paraffin was reported by Jeong and coworkers. The catalysts with the most active performance were 10 weight percent $\mathrm{Ni}_{2} \mathrm{P} / \mathrm{SiO}_{2}$ and 1 weight percent $\mathrm{Pt} / \mathrm{Al}_{2} \mathrm{O}_{3}$. Due to the decrease of $\mathrm{Pt}$ sites caused by $\mathrm{Pt}$ sintering and the presence of carbon deposits, the activity of $1 \mathrm{wt}$. percent $\mathrm{Pt} / \mathrm{Al}_{2} \mathrm{O}_{3}$ varied. Due to the reduced production of carbon deposits, $10 \mathrm{wt}$. percent $\mathrm{Ni}_{2} \mathrm{P} / \mathrm{SiO}_{2}$ displayed a more consistent performance. Escalona and coworkers reported on the use of a sulfided $\mathrm{Ni}-\mathrm{Mo} /$ $\mathrm{SiO}_{2}-\mathrm{Al}_{2} \mathrm{O}_{3}$ catalyst for the hydroprocessing reaction of light cycle oil in a fixed bed reactor. The catalyst might reduce polyaromatic hydrocarbon compounds from $64 \%$ to $5 \%$. They observed the conversion of 3-ring polyaromatic 
compounds to alkyl-substituted tetralin compounds using GC-MS studies. Also, because of the higher-octane number, the production of the aromatic increased by roughly 4-6 times, which may be appropriate for the gasoline pool. The increased octane number was also attributable to a $35-55 \%$ monoaromatic hydrocarbon concentration in hydroprocessed products under all operating conditions. The catalyst's advantage was owing to its mesoporous characteristics' resistance to pore-blocking throughout the reaction [234].

4.4. Process Parameters. Optimum conditions that led to the high efficiency of the nanocatalysts used in the three domains have been explained in the following section to guide the researchers in their future endeavors.

Supports with large pores and multiple active sites have been reported to increase the efficiency of the catalysts. The surface of supports can be functionalized and modified to act as cocatalyst, making it more cost-efficient to combat the additional cost of loading nanoparticles onto the support. Optimum results are observed to take place when green chemistry conditions are followed. The catalyst should be recyclable, easy to recover, and reuse. A catalyst that can have multiple distinct active sites is ideal since it can be applied in a one-pot synthesis. If one catalyst is used that catalyzes multiple reactions taking place in the synthesis process, then there will be no problems arising due to differences in the coordination chemistry of multiple catalysts being used in one pot, driving up the efficiency. Stable catalysts requiring a mild and benign environment are preferred since they reduce or prevent the corrosion of the production equipment. The catalysts must have good thermal and hydrolytic stability to avoid losing active sites due to leaching. Loss of active sites reduces the catalytic activity and ultimately causes catalyst deactivation [348].

MOFs can be modified with ligands that improve stereocontrol and hydrolytic and thermal stability to avoid leaching in the presence of acids or bases, which reduces the number of catalyst sites and deactivates catalysts. Leaching also increases the amount of metal in pharmaceutical products, which should otherwise strictly lie between the allowed limits for metal contamination [164]. MOFs modified with chiral ligands can be used in asymmetric synthesis since they have enantioselectivities as high as $99 \%$ with appropriate conditions and ligands. Research can be done to upscale these reactions and use them in industrial reactions, along with fixing some of their drawbacks, including leaching, improving their TON and stability, increasing their pore size, and finally, improving the versatility of their applications [349].

Mott-Schottky effect taking place in N-doped mesoporous carbon optimized their efficiency, and the reaction had a 93.2\% conversion rate. Hydrotalcite is a suitable substitute for alkaline homogeneous bases in organic synthesis. They enable environmentally benign processes with easy reuse of the catalyst. They can be assisted with ultrasonic or microwave processes to promote a benign, polar solvent-free reaction. They are cheap, have good selectivity, and have versatile applications. Mg-Al hydrotalcite has been reported by Basahel et al. [14] to be a better catalyst than other solid bases. They can be assisted with ultrasonic or microwave irradiations for a polar solvent-free reaction under mild conditions. Hydrotalcite reduces the corrosion but does not eliminate it.

It can be useful to note that using ultrasonic radiationassisted catalytic reactions saves the energy that would be used for heating for increasing the temperature. They also reduce the reaction time, saving a lot of time. Ultrasonic irradiation of catalysts improves their mass transfer rates and increases the surface area of the catalysts available for the reactants by ultrasonic dispersion. There have been cases where this irradiation also initiated reactions that do not take place in silent conditions [14]. Microwave-assisted catalytic reactions allow faster, solvent-free reactions with higher yields. They can be used in green reactions using water as a solvent to improve the solubility of hydrophobic reactants and products [350].

\section{Summary}

Nanomaterials possess dominant optical, electrical, and mechanical properties. As a result, they find extensive functions in various fields, including electronic, magnetic, and optoelectronic, biomedical, pharmaceutical, cosmetic, energy, catalytic, and materials. Nanomaterials are finding a large number of applications in catalytic reactions owing to a great number of surface atoms and several other unique characteristics. In the case of metal nanoparticles, preferential binding between clusters of metal particles and edge structures in carbon nanofiber changes the particle strain, thus tuning the electronic characteristics of the metallic nanoparticles. Additionally, defect engineering is utilized for increasing the number of reaction sites during electrocatalysis by creating active grain boundaries in TMO (transition metal oxides).

Fine chemicals are pure substances with high added value manufactured in comparatively small amounts that can be classified into active pharmaceutical ingredients (API), agrochemicals, and high-purity specialty chemicals. Fine chemical synthesis industries produce a lot of waste, and their production is small. Catalysts with a higher yield, selectivity, and activity are to be used to reduce the amount of waste produced. Homogeneous metal nanomaterial catalysts can be loaded onto supports which can be functionalized to increase the catalytic performance. However, they have a negative effect on the environment, which is why more fine chemical production industries are switching to greener heterogeneous catalysts. Solid catalysts are replacing organic and mineral solvents in organic synthesis reactions because of the possibility of reuse, reduction in waste, and there is no need to neutralize the products. Synthesis and application of certain catalysts used in fine chemical synthesis are provided along with their green substitutes. The wide application of palladium and its use in one-pot synthesis has been elucidated; however, since they are an expensive and precious metal, better alternatives have been found. MOFs can also be functionalized to improve the properties of the catalyst. Metal-organic frameworks 
modified with chiral ligands have good stability, accessibility of active sites, and porous structure. They have been studied and experimented with for the past twenty years, and high enantioselectivities of $99 \%$ have been achieved. However, using them in a practical scenario has not been successful in catalyzing efficient reactions because of leaching and stability issues. Green chemistry and its relationship with nanomaterials have been elucidated.

Although nanomaterials can be of various sizes and shapes, only a few can give us the desired product with all the requisite parameters. Various types of nanoparticles such as carbon nanotubes, $\mathrm{Ag} / \mathrm{Al}_{2} \mathrm{O}_{3}$ nanomaterials, $\mathrm{SrCO}_{3}$ and $\mathrm{TiO}_{2}$, $\mathrm{Ni} / \mathrm{Fe}$ nanofibers/porous carbon catalysts, monometallic $\mathrm{NiCNF} / \mathrm{PCs}$ and FeCNF/PCs catalysts, bimetallic Au/Ag and trimetallic $\mathrm{Pd}-\mathrm{Au} / \mathrm{Ag}$ core-shelled NPs, Pd-Au/Ag NPs, layered double hydroxides $(\mathrm{LDH}), \mathrm{Ni} / \mathrm{Al}_{2} \mathrm{O}_{3}$ core-shell catalysts, gamma- $\mathrm{Al}_{2} \mathrm{O}_{3}$ doped with $\mathrm{MnO}_{2}$, nano-SAPO-3 catalysts, highly-dispersed $\mathrm{Cr}_{2} \mathrm{O}_{3}-\mathrm{ZrO}_{2}$ binary oxide nanomaterials, fibrous nanosilica (KCC-1)-supported ruthenium nanoparticles, mesoporous nanoparticles, $\mathrm{TiO}_{2}$ nanofibers, HAIMSN, and many more types of catalysts were covered in this review paper. This study has emphasized more on the performance of each catalyst and its potential use in the future. The mechanisms through which the catalysts work have also been mentioned for easier understanding. It should be noted that the various catalysts analyzed in this paper have been tested in various reactions used in the hydrocarbon processing industry. Hence, a result shown by one catalyst in one particular type of reaction might not be the same for the same catalysts in other reactions. It is expected that hydrocarbon processing will become more and more dependent on nanocatalysts for its daily processes, and hence, this is a field having good research opportunities. Given the current interest in nanomaterials and the potential applications they have in the hydrocarbon processing, we are hopeful that sooner or later, we would be having efficient nanocatalysts, which would help the hydrocarbon industry greatly.

Catalytical research in the field of novel photocatalysts, electrocatalysts, electrochemical, and photoelectrochemical catalysts has been addressed in this paper, starting from reducing $\mathrm{CO}_{2}$ emissions, sequestering them, and utilizing the same in different processes for synthesizing value-added chemicals. This was followed by water splitting. Water splitting, as an innovative energy conversion technique powered by renewable energy, has gained popularity for producing clean hydrogen fuel from water. To complete the energy transfer process, HER and OER are involved, and cost-effective catalysts are necessary. Environmental treatment was the next subject of concern which mainly focused on the abatement of VOC using different catalysts. Moreover, remediation of wastewater was another important parameter that was looked upon. Given the increased dangers posed by global warming caused by the use of fossil fuels, our world must adopt new ways to harness the limitless sources of energy, which may be accomplished partially through hydrogen uptake. Furthermore, fuel cells were found to be devices that use electrochemical processes, thereby behaving as one of the major enabling technologies for establishing a transition to a renewable-based future.
Albeit there are advances in chemical production and this leading to the environmental impact reducing, there still exists this issue of sustainability. To solve this as result, a lot of progress has been made to utilize fossil fuels for energy production as well as to behave like a raw material. It is very vital to enhance the usage of renewable energy (RE) while also keeping in mind methods to valorize alternative raw materials for the same. In order words, strategies and technologies for the upcoming energy-efficient process industry are much needed.

\section{Conclusion and Future Directions}

Fine chemical synthesis requires highly selective and efficient catalysts to catalyze its reactions because of their high $\mathrm{E}$ factor. It is crucial to come up with catalysts that have practical applications, is scalable, and follows the 12 conditions set by green chemistry. A lot of progress has been made in this area. However, there is a lot of scope for improving the efficiency of the reactions by using heterogeneous catalysts in the industry instead of homogeneous catalysts and using green solvents instead of the traditional stoichiometric solvents. The research conducted in the area of fullerene and its application in fine chemical synthesis is inadequate, and more research could be allocated in this direction. There could be more efforts into scaling up asymmetrical synthesis using chiral ligand modified MOFs and overcoming some of its drawbacks. Although various catalysts have been explored in this paper, most nanocatalysts will only work or show the promised results to only one type of conversion, which has been mentioned. However, since the mechanisms, properties of these nanocatalysts, and their operating conditions have been explained, researchers can use these and try to come up with similar production techniques and come up with novel nanocatalysts for other processes. The authors of this paper believe that the combinations are endless, and hence, researchers should feel free to produce catalysts through methods that might feel unconventional for that particular process but have been successful for other processes. Nanocatalysts, in many instances, not only showed better conversion rates and selectivity but also good recyclability. Ergo, there should be more research in the field of hydrocarbon processing to unleash the maximum potential of the said catalysts.

The evolution of life on our planet is dependent on energy. However, the circumstances and availability of many energy sources have varied drastically over Earth's history. Catalysis is a crucial foundation of today's chemical industry, allowing for substantial advances in processes and technologies for the benefit of humanity in recent years. Catalyst creation is, in fact, a critical step in the development of efficient catalytic processes capable of maximizing the value of starting materials while reducing waste production and energy needs. This paper was mainly concerned with establishing mechanisms facilitating the optimum production of renewable energy. This could either be done by fabricating renewable energy from scratch, indirectly working for it by reducing toxic emissions, or sequestering 
hydrogen or carbon dioxide by different methods and employing them, in turn, for producing valuable chemicals.

\section{Data Availability}

All data are included within the manuscript.

\section{Conflicts of Interest}

The authors declare no conflicts of interest.

\section{References}

[1] M. Alle, S.-H. Lee, and J.-C. Kim, "Ultrafast synthesis of gold nanoparticles on cellulose nanocrystals via microwave irradiation and their dyes-degradation catalytic activity," Journal of Materials Science \& Technology, vol. 41, pp. 168-177, 2020.

[2] P. V. Adhyapak, S. P. Meshram, V. Tomar, D. P. Amalnerkar, and I. S. Mulla, "Effect of preparation parameters on the morphologically induced photocatalytic activities of hierarchical zinc oxide nanostructures," Ceramics International, vol. 39, no. 7, pp. 7367-7378, 2013.

[3] U. S. Rashid, T. K. Das, T. S. Sakthivel, S. Seal, and A. N. Bezbaruah, "GO-CeO 2 nanohybrid for ultra-rapid fluoride removal from drinking water," Science of the Total Environment, vol. 793, Article ID 148547, 2021.

[4] X. Cui, W. Li, P. Ryabchuk, K. Junge, and M. Beller, "Bridging homogeneous and heterogeneous catalysis by heterogeneous single-metal-site catalysts," Nature Catalysis, vol. 1, no. 6, pp. 385-397, 2018.

[5] Z. Edris and N. Mohamad, "Comparison studies on lauric acid ethoxylated using homogenous and heterogenous catalysts," Sains Malaysiana, vol. 39, no. 5, pp. 765-768, 2010.

[6] I. W. Davies, L. Matty, D. L. Hughes, and P. J. Reider, "Are heterogeneous catalysts precursors to homogeneous catalysts?" Journal of the American Chemical Society, vol. 123, no. 41, pp. 10139-10140, 2001.

[7] D. Astruc, F. Lu, and J. R. Aranzaes, "Nanoparticles as recyclable catalysts: the frontier between homogeneous and heterogeneous catalysis," Angewandte Chemie International Edition, vol. 44, no. 48, pp. 7852-7872, 2005.

[8] N. Sharma, H. Ojha, A. Bharadwaj, D. P. Pathak, and R. K. Sharma, "Preparation and catalytic applications of nanomaterials: a review," RSC Advances, vol. 5, no. 66, pp. 53381-53403, 2015.

[9] J. Choudhury, "Recent developments on artificial switchable catalysis," Tetrahedron Letters, vol. 59, no. 6, pp. 487-495, 2018.

[10] R. Francke and R. D. Little, "Redox catalysis in organic electrosynthesis: basic principles and recent developments," Chemical Society Reviews, vol. 43, no. 8, p. 2492, 2014.

[11] R. Rochat, M. J. Lopez, H. Tsurugi, and K. Mashima, "Recent developments in homogeneous organomagnesium catalysis," ChemCatChem, vol. 8, no. 1, pp. 10-20, 2015.

[12] H. Yang, H. Gao, and R. J. Angelici, "Hydrogenation of arenes under mild conditions using rhodium pyridylphosphine and bipyridyl complexes tethered to a silicasupported palladium heterogeneous catalyst," Organometallics, vol. 19, no. 4, pp. 622-629, 2000.

[13] P. Barbaro, V. D. Santo, and F. Liguori, "Emerging strategies in sustainable fine-chemical synthesis: asymmetric catalysis by metal nanoparticles," Dalton Transactions, vol. 39, no. 36, p. 8391, 2010.
[14] S. N. Basahel, S. A. Al-Thabaiti, K. Narasimharao, N. S. Ahmed, and M. Mokhtar, "Nanostructured Mg-Al hydrotalcite as catalyst for fine chemical synthesis," Journal of Nanoscience and Nanotechnology, vol. 14, no. 2, pp. 1931-1946, 2014.

[15] H. Du, F. Zhou, W. Pang, and Y. Yue, "Synthesis and characterization of titanium silicate molecular sieves with zorite-type structure," Microporous Materials, vol. 7, no. 2-3, pp. 73-80, 1996.

[16] A. Rosales, I. Rodríguez-García, J. Muñoz-Bascón et al., “The Nugent reagent: a formidable tool in contemporary radical and organometallic chemistry," European Journal of Organic Chemistry, vol. 2015, no. 21, pp. 4567-4591, 2015.

[17] S. Cheng, X. Wang, and S.-Y. Chen, "Applications of aminefunctionalized mesoporous silica in fine chemical synthesis," Topics in Catalysis, vol. 52, no. 6-7, pp. 681-687, 2009.

[18] M. Manßen and L. L. Schafer, "Titanium catalysis for the synthesis of fine chemicals-development and trends," Chemical Society Reviews, vol. 49, 2020.

[19] S. Sadjadi and M. M. Heravi, "Recent advances in applications of POMs and their hybrids in catalysis," Current Organic Chemistry, vol. 20, no. 13, pp. 1404-1444, 2016.

[20] A. Severino, J. Vital, and L. S. Lobo, "Isomerization of $\alpha$-pinene over $\mathrm{TiO}_{2}$ : kinetics and catalyst optimization," Studies in Surface Science and Catalysis, Elsevier, Amsterdam, Netherlands, pp. 685-692, 1993.

[21] S. M. Landge, M. Berryman, and B. Török, "Microwaveassisted solid acid-catalyzed one-pot synthesis of isobenzofuran-1(3H)-ones," Tetrahedron Letters, vol. 49, no. 29-30, pp. 4505-4508, 2008.

[22] B. Török, M. Abid, and O. De Paolis, "A novel one-pot synthesis of $\mathrm{N}$-acylindoles from primary aromatic amides," Synlett, vol. 2008, no. 3, pp. 410-412, 2008.

[23] Y. Zhou, L. K. Woo, and R. J. Angelici, "Solid acid catalysis of tandem isomerization-lactonization of olefinic acids," Applied Catalysis A: General, vol. 333, no. 2, pp. 238-244, 2007.

[24] R. G. Jacob, L. G. Dutra, C. S. Radatz, S. R. Mendes, G. Perin, and E. J. Lenardão, "Synthesis of 1,2-disubstitued benzimidazoles using $\mathrm{SiO}_{2} / \mathrm{ZnCl}_{2}$," Tetrahedron Letters, vol. 50, no. 13, pp. 1495-1497, 2009.

[25] G. Kuriakose, J. B. Nagy, and N. Nagaraju, "One pot synthesis of phenyl acetate and o-hydroxyacetophenone via esterification and fries rearrangement over zeolite catalysts," in Studies in Surface Science and Catalysis, vol. 154, pp. 2803-2808, Elsevier, Amsterdam, Netherlands, 2004.

[26] K. V. N. S. Srinivas and B. Das, "Microwave assisted convenient and facile regeneration of carbonyl compounds from oximes, semicarbazones and phenylhydrazones using silica supported ceric ammonium nitrate," Journal of Chemical Research, vol. 2002, no. 11, pp. 556-557, 2002.

[27] J. X. Liu, N. He, C. Y. Liu, G. R. Wang, Q. Xin, and H. C. Guo, "Engineering the porosity and acidity of $\mathrm{H}$-beta zeolite by dealumination for the production of 2-ethylanthraquinone via 2-(4'-ethylbenzoyl)benzoic acid dehydration," RSC Advances, vol. 8, no. 18, pp. 9731-9740, 2018.

[28] M. Bandini, P. G. Cozzi, M. Giacomini, P. Melchiorre, S. Selva, and A. Umani-Ronchi, "Sequential one-pot InBr3catalyzed 1,4- then 1,2-nucleophilic addition to enones," The Journal of Organic Chemistry, vol. 67, no. 11, pp. 3700-3704, 2002.

[29] A. Corma and M. Renz, "A general method for the preparation of ethers using water-resistant solid lewis acids," Angewandte Chemie International Edition, vol. 46, no. 1-2, pp. 298-300, 2007. 
[30] S. M. Sadrameli, "Thermal/catalytic cracking of liquid hydrocarbons for the production of olefins: a state-of-the-art review II: catalytic cracking review," Fuel, vol. 173, pp. 285-297, 2016.

[31] I. E. Maxwell and W. H. J. Stork, "Chapter 17 hydrocarbon processing with zeolites," in Studies in Surface Science and Catalysis, vol. 137, pp. 747-819, Elsevier, Amsterdam, Netherlands, 2001.

[32] A. Klerk, "Zeolites as catalysts for fuels refining after indirect liquefaction processes," Molecules, vol. 23, no. 1, p. 115, 2018.

[33] T. Yashima, Z. B. Wang, A. Kamo, T. Yoneda, and T. Komatsu, "Isomerization of n-hexane over platinum loaded zeolite catalysts," Catalysis Today, vol. 29, no. 1-4, pp. 279-283, 1996.

[34] A. V. Abramova, A. A. Panin, G. A. Kliger, and S. N. Khadzhiev, "Zeolite catalysts for production of synthetic highly-octane gasoline by method of FischerTropsch," 2006, https://www.researchgate.net/publication/ 289144303_Zeolite_catalysts_for_production_of_synthetic_ highly-octane_gasoline_by_method_of_Fischer-Tropsch.

[35] S. Hajimirzaee, "Preparation, modification and characterisation of selective zeolite based catalysts for petrochemical applications," Doctoral dissertation, University of Birmingham, Birmingham, UK, 2015.

[36] A. L. Lapidus, "The mechanism of hydrocarbon synthesis from $\mathrm{CO}$ and $\mathrm{H}_{2}$ on cobalt catalysts," Solid Fuel Chemistry, vol. 47, no. 6, pp. 315-328, 2013.

[37] C. P. Nicholas, "Applications of light olefin oligomerization to the production of fuels and chemicals," Applied Catalysis A: General, vol. 543, pp. 82-97, 2017.

[38] J. H. Coetzee, T. N. Mashapa, N. M. Prinsloo, and J. D. Rademan, "An improved solid phosphoric acid catalyst for alkene oligomerization in a Fischer-Tropsch refinery," Applied Catalysis A: General, vol. 308, pp. 204-209, 2006.

[39] B. M. Antunes, A. E. Rodrigues, Z. Lin, I. Portugal, and C. M. Silva, "Alkenes oligomerization with resin catalysts," Fuel Processing Technology, vol. 138, pp. 86-99, 2015.

[40] D. McGuinness, "Alkene oligomerisation and polymerisation with metal-NHC based catalysts," Dalton Transactions, vol. 35, pp. 6915-6923, 2009.

[41] G. Centi, E. A. Quadrelli, and S. Perathoner, "Catalysis for $\mathrm{CO}_{2}$ conversion: a key technology for rapid introduction of renewable energy in the value chain of chemical industries," Energy \& Environmental Science, vol. 6, no. 6, 2013.

[42] M. Selva and R. Luque, "Benign-by design advanced nanomaterials for environmental and energy-related applications," Current Opinion in Green and Sustainable Chemistry, vol. 15, 2019.

[43] P. Gómez-López, A. Puente-Santiago, A. Castro-Beltrán et al., "Nanomaterials and catalysis for green chemistry," Current Opinion in Green and Sustainable Chemistry, vol. 24, pp. 48-55, 2020.

[44] X. Zhang, P. Zhang, C. Chen et al., "Fabrication of 2D metalorganic framework nanosheets with tailorable thickness using bio-based surfactants and their application in catalysis," Green Chemistry, vol. 21, no. 1, pp. 54-58, 2019.

[45] H. Koempel and W. Liebner, "Lurgi's methanol to propylene (MTP) report on a successful commercialisation," in Studies in Surface Science and Catalysis, vol. 167, pp. 261-267, Elsevier, Amsterdam, Netherlands, 2007.

[46] X.-M. Liu, G. Q. Lu, Z.-F. Yan, and J. Beltramini, "Recent advances in catalysts for methanol synthesis via hydrogenation of $\mathrm{CO}$ and $\mathrm{CO}_{2}$," Industrial \& Engineering Chemistry Research, vol. 42, no. 25, pp. 6518-6530, 2003.
[47] P. H. Dixneuf, “A bridge from $\mathrm{CO}_{2}$ to methanol," Nature chemistry, vol. 3, no. 8, pp. 578-579, 2011.

[48] R. W. Dorner, D. R. Hardy, F. W. Williams, and H. D. Willauer, "C2-C5+ olefin production from $\mathrm{CO}_{2}$ hydrogenation using ceria modified $\mathrm{Fe} / \mathrm{Mn} / \mathrm{K}$ catalysts," $\mathrm{Ca}$ talysis Communications, vol. 15, no. 1, pp. 88-92, 2011.

[49] F. Tihay, A. C. Roger, G. Pourroy, and A. Kiennemann, "Role of the alloy and spinel in the catalytic behavior of $\mathrm{Fe}-\mathrm{Co} /$ cobalt magnetite composites under $\mathrm{CO}$ and $\mathrm{CO}_{2}$ hydrogenation," Energy \& Fuels, vol. 16, no. 5, pp. 1271-1276, 2002.

[50] S. Tada, T. Shimizu, H. Kameyama, T. Haneda, and R. Kikuchi, "Ni/CeO 2 catalysts with high $\mathrm{CO}_{2}$ methanation activity and high $\mathrm{CH}_{4}$ selectivity at low temperatures," International Journal of Hydrogen Energy, vol. 37, no. 7, pp. 5527-5531, 2012.

[51] P. G. Jessop, T. Ikariya, and R. Noyori, "Homogeneous catalytic hydrogenation of supercritical carbon dioxide," Nature, vol. 368, no. 6468, pp. 231-233, 1994.

[52] C. Y. Lin, D. Zhang, Z. Zhao, and Z. Xia, "Covalent organic framework electrocatalysts for clean energy conversion," Advanced Materials, vol. 30, no. 5, Article ID 1703646, 2018.

[53] Q. Wang, T. Hisatomi, Q. Jia et al., "Scalable water splitting on particulate photocatalyst sheets with a solar-to-hydrogen energy conversion efficiency exceeding 1\%," Nature Materials, vol. 15, no. 6, pp. 611-615, 2016.

[54] R. Ma, J. Sun, D. H. Li, and J. J. Wei, "Review of synergistic photo-thermocatalysis: mechanisms, materials and applications," International Journal of Hydrogen Energy, vol. 45, 2020.

[55] F. Janssen, "Catalysis for renewable energy and chemicals, the thermal conversion of biomass," Environmental Catalysis, vol. 1, pp. 15-36, 1999.

[56] X. Zou and Y. Zhang, "Noble metal-free hydrogen evolution catalysts for water splitting," Chemical Society Reviews, vol. 44, no. 15, pp. 5148-5180, 2015.

[57] J.-W. Wang, W.-J. Liu, D.-C. Zhong, and T.-B. Lu, "Nickel complexes as molecular catalysts for water splitting and $\mathrm{CO}_{2}$ reduction," Coordination Chemistry Reviews, vol. 378, pp. 237-261, 2019.

[58] C. Jiang, D. Li, P. Zhang, J. Li, J. Wang, and J. Yu, "Formaldehyde and volatile organic compound (VOC) emissions from particleboard: identification of odorous compounds and effects of heat treatment," Building and Environment, vol. 117, pp. 118-126, 2017.

[59] K. N. Harish, H. S. Bhojya Naik, P. N. Prashanth Kumar, and R. Viswanath, "Optical and photocatalytic properties of solar light active Nd-substituted $\mathrm{Ni}$ ferrite catalysts: for environmental protection," ACS Sustainable Chemistry \& Engineering, vol. 1, no. 9, pp. 1143-1153, 2013.

[60] K. Fujiwara, K. Okuyama, and S. E. Pratsinis, "Metal-support interactions in catalysts for environmental remediation," Environmental Science: Nano, vol. 4, no. 11, pp. 2076-2092, 2017.

[61] Y. Fang and Y. Guo, "Copper-based non-precious metal heterogeneous catalysts for environmental remediation," Chinese Journal of Catalysis, vol. 39, no. 4, pp. 566-582, 2018.

[62] U. Chadha, S. K. Selvaraj, S. V. Thanu et al., "A review of the function of using carbon nanomaterials in membrane filtration for contaminant removal from wastewater," Materials Research Express, in Press, 2021.

[63] R. Bashyam and P. Zelenay, "A class of non-precious metal composite catalysts for fuel cells," in Materials for Sustainable Energy, pp. 247-250, Nature Publishing Group, Berlin, Germany, 2011. 
[64] P. Serp and J. L. Figueiredo, Carbon Materials for Catalysis, Wiley, Hoboken, NJ, USA, 2009.

[65] J. Zhu, A. Holmen, and D. Chen, "Carbon nanomaterials in catalysis: proton affinity, chemical and electronic properties, and their catalytic consequences," ChemCatChem, vol. 5, no. 2, pp. 378-401, 2013.

[66] K. L. Klein, A. V. Melechko, T. E. McKnight et al., "Surface characterization and functionalization of carbon nanofibers," Journal of Applied Physics, vol. 103, no. 6, Article ID 061301, 2008.

[67] N. M. Rodriguez, "A review of catalytically grown carbon nanofibers," Journal of Materials Research, vol. 8, no. 12, pp. 3233-3250, 1993.

[68] L. Radovic and F. Rodriguez-Teinoso, Chemistry, Physics of Carbon, Vol. 25, Marcel Dekker, , New York, NY, USA, 1997.

[69] P. Serp, "Carbon nanotubes and nanofibers in catalysis," Applied Catalysis A: General, vol. 253, no. 2, pp. 337-358, 2003.

[70] I. Kvande, J. Zhu, T.-J. Zhao et al., "Importance of oxygenfree edge and defect sites for the immobilization of colloidal Pt oxide particles with implications for the preparation of CNF-supported catalysts," The Journal of Physical Chemistry C, vol. 114, no. 4, pp. 1752-1762, 2010.

[71] A. Valtanen, M. Huuhtanen, A.-R. Rautio, T. Kolli, K. Kordás, and R. L. Keiski, "Noble metal/CNT based catalysts in $\mathrm{NH}_{3}$ and EtOH assisted SCR of NO," Topics in Catalysis, vol. 58, no. 14-17, pp. 984-992, 2015.

[72] M. Toebes, Y. Zhang, J. Hájek et al., "Support effects in the hydrogenation of cinnamaldehyde over carbon nanofibersupported platinum catalysts: characterization and catalysis," Journal of Catalysis, vol. 226, no. 1, pp. 215-225, 2004.

[73] H. Vu, F. Goncalves, R. Philippe et al., "Bimetallic catalysis on carbon nanotubes for the selective hydrogenation of cinnamaldehyde," Journal of Catalysis, vol. 240, no. 1, pp. 18-22, 2006.

[74] C.-H. Li, Z.-X. Yu, K.-F. Yao, S.-f. Ji, and J. Liang, "Nitrobenzene hydrogenation with carbon nanotube-supported platinum catalyst under mild conditions," Journal of Molecular Catalysis A: Chemical, vol. 226, no. 1, pp. 101-105, 2005.

[75] G. Ovejero, J. L. Sotelo, M. D. Romero et al., "Multiwalled carbon nanotubes for liquid-phase oxidation. functionalization, characterization, and catalytic activity," Industrial \& Engineering Chemistry Research, vol. 45, no. 7, pp. 2206-2212, 2006.

[76] D. B. Thakur, R. M. Tiggelaar, T. M. C. Hoang, J. G. E. Gardeniers, L. Lefferts, and K. Seshan, "Ruthenium catalyst on carbon nanofiber support layers for use in siliconbased structured microreactors, part I: preparation and characterization," Applied Catalysis B: Environmental, vol. 102, no. 1-2, pp. 232-242, 2011.

[77] G. Wu and B.-Q. Xu, "Carbon nanotube supported Pt electrodes for methanol oxidation: a comparison between multi- and single-walled carbon nanotubes," Journal of Power Sources, vol. 174, no. 1, pp. 148-158, 2007.

[78] J. Zhu, T. Zhao, I. Kvande, D. Chen, X. Zhou, and W. Yuan, "Carbon nanofiber-supported Pd catalysts for heck reaction: effects of support interaction," Chinese Journal of Catalysis, vol. 29, no. 11, pp. 1145-1151, 2008.

[79] A. B. Garćia, A. Cuesta, M. A. Montes-Morán, A. MartínezAlonso, and J. M. D. Tascón, "Zeta potential as a tool to characterize plasma oxidation of carbon fibers," Journal of Colloid and Interface Science, vol. 192, no. 2, pp. 363-367, 1997.
[80] F. Rodríguez-reinoso, "The role of carbon materials in heterogeneous catalysis," Carbon, vol. 36, no. 3, pp. 159-175, 1998.

[81] W. A. Spieker and J. R. Regalbuto, "A fundamental model of platinum impregnation onto alumina," Chemical Engineering Science, vol. 56, no. 11, pp. 3491-3504, 2001.

[82] D. C. Elliott, L. J. Sealock, and E. G. Baker, "Chemical processing in high-pressure aqueous environments. 2. Development of catalysts for gasification," Industrial \& Engineering Chemistry Research, vol. 32, no. 8, pp. 1542-1548, 1993.

[83] P.-X. Hou, H. Orikasa, T. Yamazaki et al., "Synthesis of nitrogen-containing microporous carbon with a highly ordered structure and effect of nitrogen doping on $\mathrm{H}_{2} \mathrm{O}$ adsorption," Chemistry of Materials, vol. 17, no. 20, pp. 5187-5193, 2005.

[84] A. Zamudio, A. L. Elías, J. A. Rodríguez-Manzo et al., "Efficient anchoring of silver nanoparticles on $\mathrm{N}$-doped carbon nanotubes,” Small, vol. 2, no. 3, pp. 346-350, 2006.

[85] Z. Zhao, M. Li, L. Zhang, L. Dai, and Z. Xia, "Design principles for heteroatom-doped carbon nanomaterials as highly efficient catalysts for fuel cells and metal-air batteries," Advanced Materials, vol. 27, no. 43, pp. 6834-6840, 2015.

[86] M. Scardamaglia and C. Bittencourt, "Metal-free catalysis based on nitrogen-doped carbon nanomaterials: a photoelectron spectroscopy point of view," Beilstein Journal of Nanotechnology, vol. 9, pp. 2015-2031, 2018.

[87] C. Zhu and S. Dong, "Recent progress in graphene-based nanomaterials as advanced electrocatalysts towards oxygen reduction reaction," Nanoscale, vol. 5, no. 5, p. 1753, 2013.

[88] R. Xiao, Z. Luo, Z. Wei et al., "Activation of peroxymonosulfate/persulfate by nanomaterials for sulfate radical-based advanced oxidation technologies," Current Opinion in Chemical Engineering, vol. 19, pp. 51-58, 2018.

[89] I. Suarez-Martinez, C. Bittencourt, X. Ke et al., "Probing the interaction between gold nanoparticles and oxygen functionalized carbon nanotubes," Carbon, vol. 47, no. 6, pp. 1549-1554, 2009.

[90] T.-J. Zhao, D. Chen, Y.-C. Dai, W.-K. Yuan, and A. Holmen, "The effect of graphitic platelet orientation on the properties of carbon nanofiber supported Pd catalysts prepared by ion exchange," Topics in Catalysis, vol. 45, no. 1-4, pp. 87-91, 2007.

[91] I. Efremenko and M. Sheintuch, "Carbon-supported palladium catalysts. Molecular orbital study," Journal of Catalysis, vol. 214, no. 1, pp. 53-67, 2003.

[92] A. Chambers, T. Nemes, N. M. Rodriguez, and R. T. K. Baker, "Catalytic behavior of graphite nanofiber supported nickel particles. 1. Comparison with other support media," The Journal of Physical Chemistry B, vol. 102, no. 12, pp. 2251-2258, 1998.

[93] S. Latil, S. Roche, D. Mayou, and J. C. Charlier, "Mesoscopic transport in chemically doped carbon nanotubes," Physical Review Letters, vol. 92, no. 25, Article ID 256805, 2004.

[94] C. Fang, L. Liu, J. Weng et al., "Modifiers vs. channels: creating shape-selective catalysis of metal nanoparticles/ porous-nanomaterials," Angewandte Chemie International Edition, vol. 133, 2020.

[95] V. V. Chesnokov, I. P. Prosvirin, N. A. Zaitseva, V. I. Zaikovskii, and V. V. Molchanov, "Effect of the structure of carbon nanofibers on the state of an active component and on the catalytic properties of $\mathrm{Pd} / \mathrm{C}$ catalysts in the selective hydrogenation of 1,3-butadiene," Kinetics and Catalysis, vol. 43, no. 6, pp. 838-846, 2002. 
[96] M. Ruta, N. Semagina, and L. Kiwi-Minsker, "Monodispersed Pd nanoparticles for acetylene selective hydrogenation: particle size and support effects," The Journal of Physical Chemistry C, vol. 112, no. 35, pp. 13635-13641, 2008.

[97] E. Asedegbega-Nieto, B. Bachiller-Baeza, D. G. Kuvshinov et al., "Effect of the carbon support nano-structures on the performance of $\mathrm{Ru}$ catalysts in the hydrogenation of paracetamol," Carbon, vol. 46, no. 7, pp. 1046-1052, 2008.

[98] C. Wang, J. Qiu, C. Liang, L. Xing, and X. Yang, "Carbon nanofiber supported $\mathrm{Ni}$ catalysts for the hydrogenation of chloronitrobenzenes," Catalysis Communications, vol. 9, no. 8, pp. 1749-1753, 2008.

[99] F. Salman, C. Park, and R. T. K. Baker, "Hydrogenation of crotonaldehyde over graphite nanofiber supported nickel," Catalysis Today, vol. 53, no. 3, pp. 385-394, 1999.

[100] S. Takenaka, T. Iguchi, E. Tanabe, H. Matsune, and M. Kishida, "Catalytic performance of Pt metal particles at the tips of carbon nanotubes," Catalysis Letters, vol. 141, no. 6, pp. 821-825, 2011.

[101] A. Solhy, B. F. Machado, J. Beausoleil et al., "MWCNT activation and its influence on the catalytic performance of $\mathrm{Pt} /$ MWCNT catalysts for selective hydrogenation," Carbon, vol. 46, no. 9, pp. 1194-1207, 2008.

[102] A. J. Plomp, H. Vuori, A. O. I. Krause, K. P. de Jong, and J. H. Bitter, "Particle size effects for carbon nanofiber supported platinum and ruthenium catalysts for the selective hydrogenation of cinnamaldehyde," Applied Catalysis A: General, vol. 351, no. 1, pp. 9-15, 2008.

[103] Z.-T. Liu, C.-X. Wang, Z.-W. Liu, and J. Lu, "Selective hydrogenation of cinnamaldehyde over Pt-supported multiwalled carbon nanotubes: insights into the tube-size effects," Applied Catalysis A: General, vol. 344, no. 1-2, pp. 114-123, 2008.

[104] X. Li, J. Liu, Y. Zhang et al., "High concentration nitrogen doped carbon nanotube anodes with superior $\mathrm{Li}+$ storage performance for lithium rechargeable battery application," Journal of Power Sources, vol. 197, pp. 238-245, 2012.

[105] B. Ni and X. Wang, "Face the edges: catalytic active sites of nanomaterials," Advanced Science, vol. 2, no. 7, Article ID $1500085,2015$.

[106] J. Zhu, Y. Huang, W. Mei et al., "Effects of intrinsic pentagon defects on electrochemical reactivity of carbon nanomaterials," Angewandte Chemie, vol. 58, 2019.

[107] A. S. Nia, S. Rana, D. Döhler et al., "Carbon-supported copper nanomaterials: recyclable catalysts for Huisgen [3+2] cycloaddition reactions," Chemistry-A European Journal, vol. 21, no. 30, pp. 10763-10770, 2015.

[108] B. Zhang, Z. Qi, Z. Wu et al., "Defect-rich 2D material networks for advanced oxygen evolution catalysts," ACS Energy Letters, vol. 4, no. 1, pp. 328-336, 2018.

[109] R. Zhang, K. Lu, L. Zong et al., "Control synthesis of $\mathrm{CeO}_{2}$ nanomaterials supported gold for catalytic oxidation of carbon monoxide," Molecular Catalysis, vol. 442, pp. 173-180, 2017.

[110] Z. Wang, Y. Zou, Y. Li, and Y. Cheng, "Metal-containing polydopamine nanomaterials: catalysis, energy, and theranostics," Small (Weinheim an der Bergstrasse, Germany), vol. 16, no. 18, Article ID e1907042, 2020.

[111] J. Zhang and C. M. Li, "Nanoporous metals: fabrication strategies and advanced electrochemical applications in catalysis, sensing and energy systems," Chemical Society Reviews, vol. 41, no. 21, p. 7016, 2012.

[112] D. Yu, E. Nagelli, F. Du, and L. Dai, "Metal-free carbon nanomaterials become more active than metal catalysts and last longer," The Journal of Physical Chemistry Letters, vol. 1, no. 14, pp. 2165-2173, 2010.

[113] H. Yin and Z. Tang, "Ultrathin two-dimensional layered metal hydroxides: an emerging platform for advanced catalysis, energy conversion and storage," Chemical Society Reviews, vol. 45, no. 18, pp. 4873-4891, 2016.

[114] Z.-P. Wu, S. Shan, S.-Q. Zang, and C.-J. Zhong, "Dynamic core-shell and alloy structures of multimetallic nanomaterials and their catalytic synergies," Accounts of Chemical Research, vol. 53, 2020.

[115] J. Wang and C.-j. Liu, "Preparation of $2 \mathrm{D} \mathrm{WO}_{3}$ nanomaterials with enhanced catalytic activities: current status and perspective," ChemBioEng Reviews, vol. 2, no. 5, pp. 335-350, 2015.

[116] O. V. Vodyankina, G. V. Mamontov, V. V. Dutov, T. S. Kharlamova, and M. A. Salaev, "Ag-containing nanomaterials in heterogeneous catalysis: advances and recent trends," Advanced Nanomaterials for Catalysis and Energy, Elsevier, Amsterdam, Netherlands, pp. 143-175, 2019.

[117] P. Sudarsanam, B. Hillary, B. Mallesham et al., "Designing $\mathrm{CuO}_{\mathrm{x}}$ nanoparticle-decorated $\mathrm{CeO}_{2}$ nanocubes for catalytic soot oxidation: role of the nanointerface in the catalytic performance of heterostructured nanomaterials," Langmuir, vol. 32, no. 9, pp. 2208-2215, 2016.

[118] K. Skrodczky, M. M. Antunes, X. Han et al., "Niobium pentoxide nanomaterials with distorted structures as efficient acid catalysts," Communications Chemistry, vol. 2, no. 1, 2019.

[119] R. Bhandari and M. R. Knecht, "Effects of the material structure on the catalytic activity of peptide-templated Pd nanomaterials," ACS Catalysis, vol. 1, no. 2, pp. 89-98, 2011.

[120] Y. Zou, J. M. Wheeler, H. Ma, P. Okle, and R. Spolenak, "Nanocrystalline high-entropy alloys: a new paradigm in high-temperature strength and stability," Nano Letters, vol. 17, no. 3, pp. 1569-1574, 2017.

[121] X. Li, Y. Pan, H. Yi et al., "Mott-Schottky effect leads to alkynes semi-hydrogenation over Pd-nanocubes@N-doped carbon," ACS Catalysis, vol. 9, 2019.

[122] Z.-F. Jiao, Y.-M. Tian, B. Zhang et al., "High photocatalytic activity of a $\mathrm{NiO}$ nanodot-decorated $\mathrm{Pd} / \mathrm{SiC}$ catalyst for the Suzuki-Miyaura cross-coupling of aryl bromides and chlorides in air under visible light," Journal of Catalysis, vol. 389, pp. 517-524, 2020.

[123] K. Wang, X. Tian, J. H. Jordan, K. Velmurugan, L. Wang, and X. Y. Hu, "The emerging applications of pillararene architectures in supramolecular catalysis," Chinese Chemical Letters, vol. 33, pp. 86-95, 2021.

[124] M. J. Muñoz-Batista, D. Rodríguez-Padrón, A. R. P. Santiago, and R. Luque, "Mechanochemistry: toward sustainable design of advanced nanomaterials for electrochemical energy storage and catalytic applications," ACS Sustainable Chemistry \& Engineering, vol. 6, 2018.

[125] M. Al-Naji, A. M. Balu, A. Roibu et al., "Mechanochemical preparation of advanced catalytically active bifunctional Pdcontaining nanomaterials for aqueous phase hydrogenation," Catalysis Science \& Technology, vol. 5, no. 4, pp. 2085-2091, 2015.

[126] T. Chen and V. O. Rodionov, "Controllable catalysis with nanoparticles: bimetallic alloy systems and surface adsorbates," ACS Catalysis, vol. 6, no. 6, pp. 4025-4033, 2016.

[127] K. Lakshmi and R. Rangasamy, "Synthesis of Au nanoparticles using magnetite cored polyamine dendrimer 
template and its green catalysts for selective alcohol oxidation of alcohol," Materials Letters, vol. 301, Article ID 130257, 2021.

[128] X. Liu, B. Tang, J. Long, W. Zhang, X. Liu, and Z. Mirza, “The development of MOFs-based nanomaterials in heterogeneous organocatalysis," Science Bulletin, vol. 63, no. 8, pp. 502-524, 2018.

[129] H. Konnerth, B. M. Matsagar, S. S. Chen, M. H. G. Prechtl, F.-K. Shieh, and K. C.-W. Wu, "Metal-organic framework (MOF)-derived catalysts for fine chemical production," Coordination Chemistry Reviews, vol. 416, Article ID 213319, 2020.

[130] J. A. Widegren and R. G. Finke, “Anisole hydrogenation with well-characterized polyoxoanion- and tetrabutylammonium-stabilized $\mathrm{Rh}(0)$ nanoclusters: effects of added water and acid, plus enhanced catalytic rate, lifetime, and partial hydrogenation selectivity," Inorganic Chemistry, vol. 41, no. 6, pp. 1558-1572, 2002.

[131] X. Wu, X. Li, W. Hems, F. King, and J Xiao, "Accelerated asymmetric transfer hydrogenation of aromatic ketones in water," Organic \& Biomolecular Chemistry, vol. 2, no. 13, pp. 1818-1821, 2004.

[132] Z. Li, S. Wu, C. Yang et al., "Nano- $\mathrm{CO}_{3} \mathrm{O}_{4}$ supported on magnetic $\mathrm{N}$-doped graphene as highly efficient catalyst for epoxidation of alkenes," Molecular Catalysis, vol. 432, pp. 267-273, 2017.

[133] S. Andreescu, J. Njagi, C. Ispas, and M. T. Ravalli, "JEM spotlight: applications of advanced nanomaterials for environmental monitoring," Journal of Environmental Monitoring, vol. 11, no. 1, pp. 27-40, 2009.

[134] C. V. Galliford and K. A. Scheidt, "Pyrrolidinyl-spirooxindole natural products as inspirations for the development of potential therapeutic agents," Angewandte Chemie International Edition, vol. 46, no. 46, pp. 8748-8758, 2007.

[135] M. L. Kantam, S. Laha, J. Yadav, B. M. Choudary, and B. Sreedhar, "Nanocrystalline titanium (IV) oxide as an efficient heterogeneous catalyst for Tandem Michael and nucleophilic 1, 2-addition to enones," Advanced Synthesis \& Catalysis, vol. 348, no. 7-8, pp. 867-872, 2006.

[136] M. E. Mahmoud, M. S. Abdelwahab, and E. M. Fathallah, "Design of novel nano-sorbents based on nano-magnetic iron oxide-bound-nano-silicon oxide-immobilized-triethylenetetramine for implementation in water treatment of heavy metals," Chemical Engineering Journal, vol. 223, pp. 318-327, 2013.

[137] R. Palos, A. Gutiérrez, J. M. Arandes, and J Bilbao, "Catalyst used in fluid catalytic cracking (FCC) unit as a support of NiMoP catalyst for light cycle oil hydroprocessing," Fuel, vol. 216, pp. 142-152, 2018.

[138] C. Karthikeyan, K. Varaprasad, S. K. Venugopal, S. Shakila, B. R. Venkatraman, and R. Sadiku, "Biocidal (bacterial and cancer cells) activities of chitosan/CuO nanomaterial, synthesized via a green process," Carbohydrate Polymers, vol. 259, Article ID 117762, 2021.

[139] X. Chen, Y.-S. Jun, K. Takanabe et al., "Ordered mesoporous SBA-15 type graphitic carbon nitride: a semiconductor host structure for photocatalytic hydrogen evolution with visible light," Chemistry of Materials, vol. 21, no. 18, pp. 4093-4095, 2009.

[140] X.-H. Li and M. Antonietti, "Metal nanoparticles at mesoporous N-doped carbons and carbon nitrides: functional Mott-Schottky heterojunctions for catalysis," Chemical Society Reviews, vol. 42, no. 16, p. 6593, 2013.

[141] A. Vinu, "Two-dimensional hexagonally-ordered mesoporous carbon nitrides with tunable pore diameter, surface area and nitrogen content," Advanced Functional Materials, vol. 18, no. 5, pp. 816-827, 2008.

[142] T. L. Pavlovska, R. G. Redkin, V. V. Lipson, and D. V. Atamanuk, "Molecular diversity of spirooxindoles. Synthesis and biological activity," Molecular Diversity, vol. 20, no. 1, pp. 299-344, 2015.

[143] L. Moradi and Z. Ataei, "Efficient and green pathway for onepot synthesis of spirooxindoles in the presence of $\mathrm{CuO}$ nanoparticles," Green Chemistry Letters and Reviews, vol. 10, no. 4, pp. 380-386, 2017.

[144] B. Karmakar, A. Nayak, and J. Banerji, "A clean and expedient synthesis of spirooxindoles in aqueous media catalyzed over nanocrystalline MgO," Tetrahedron Letters, vol. 53, no. 37, pp. 5004-5007, 2012.

[145] G. Bhaskar, Y. Arun, C. Balachandran, C. Saikumar, and P. T. Perumal, "Synthesis of novel spirooxindole derivatives by one pot multicomponent reaction and their antimicrobial activity," European Journal of Medicinal Chemistry, vol. 51, pp. 79-91, 2012.

[146] G. Wu, L. Ouyang, J. Liu et al., "Synthesis of novel spirooxindolo-pyrrolidines, pyrrolizidines, and pyrrolothiazoles via a regioselective three-component [3+2] cycloaddition and their preliminary antimicrobial evaluation," Molecular Diversity, vol. 17, no. 2, pp. 271-283, 2013.

[147] F. Alemi-Tameh, J. Safaei-Ghomi, M. Mahmoudi-Hashemi, and $\mathrm{M}$ Monajjemi, "Amino functionalized nano $\mathrm{Fe}_{3} \mathrm{O}_{4} @$ $\mathrm{SiO}_{2}$ as a magnetically green catalyst for the one-pot synthesis of spirooxindoles under mild conditions," Polycyclic Aromatic Compounds, vol. 38, no. 3, pp. 199-212, 2018.

[148] A.-H. Lu, E. L. Salabas, and F. Schüth, "Magnetic nanoparticles: synthesis, protection, functionalization, and application," Angewandte Chemie International Edition, vol. 46, no. 8, pp. 1222-1244, 2007.

[149] S. V. Jadhav, K. M. Jinka, and H. C Bajaj, "Nanosized sulfated zinc ferrite as catalyst for the synthesis of nopol and other fine chemicals," Catalysis today, vol. 198, no. 1, pp. 98-105, 2012.

[150] L. Wu, Y.-M. He, and Q.-H. Fan, "Controlled reversible anchoring of $\eta 6$-Arene/TsDPEN- ruthenium(II) complex onto magnetic nanoparticles: a new strategy for catalyst separation and recycling," Advanced Synthesis \& Catalysis, vol. 353, no. 16, pp. 2915-2919, 2011.

[151] Z. Cai, Q. Yao, X. Chen, and X. Wang, "Nanomaterials with different dimensions for electrocatalysis," in Novel Nanomaterials for Biomedical, Environmental, and Energy Applications, pp. 435-464, Elsevier, Amsterdam, Netherlands, 2019.

[152] F. Liu, K. Huang, A. Zheng, F.-S. Xiao, and S. Dai, "Hydrophobic solid acids and their catalytic applications in green and sustainable chemistry," ACS Catalysis, vol. 8, no. 1, pp. 372-391, 2018.

[153] B. Jarrais, A. Guedes, and C. Freire, "Heteroatom-doped carbon nanomaterials as metal-free catalysts for the reduction of 4-nitrophenol," ChemistrySelect, vol. 3, no. 6, pp. 1737-1748, 2018.

[154] A. C. Kresge, M. E. Leonowicz, W. J. Roth, J. C. Vartuli, and J. S Beck, "Ordered mesoporous molecular sieves synthesized by a liquid-crystal template mechanism," Nature, vol. 359, no. 6397, pp. 710-712, 1992.

[155] J. W. Ko, J. Li, A. Suzuki, K. Takai, and W. B. Ko, "Reduction of 2-nitrophenol using a hybrid $\mathrm{C}$-Ni nanocomposite as a catalyst," Micro \& Nano Letters, vol. 13, no. 9, pp. 1310-1314, 2018.

[156] N. Zhang, M.-Q. Yang, S. Liu, Y. Sun, and Y.-J. Xu, "Waltzing with the versatile platform of graphene to 
synthesize composite photocatalysts," Chemical Reviews, vol. 115, no. 18, pp. 10307-10377, 2015.

[157] V. Farina, J. T. Reeves, C. H. Senanayake, and J. J. Song, "Asymmetric synthesis of active pharmaceutical ingredients," Chemical Reviews, vol. 106, no. 7, pp. 2734-2793, 2006.

[158] H. U. Blaser and H. J. Federsel, Asymmetric Catalysis on Industrial Scale: Challenges, Approaches and Solutions, Wiley-VCH, Weinheim, Germany, 2nd edition, 2010.

[159] V. Hornillos, M. Pérez, M. Fañanás-Mastral, and B. L. Feringa, "Copper-catalyzed enantioselective allyl-allyl cross-coupling," Journal of the American Chemical Society, vol. 135, no. 6, pp. 2140-2143, 2013.

[160] K. P. Bryliakov, "Catalytic asymmetric oxygenations with the environmentally benign oxidants $\mathrm{H}_{2} \mathrm{O}_{2}$ and $\mathrm{O}_{2}$," Chemical reviews, vol. 117, no. 17, pp. 11406-11459, 2017.

[161] Z. Zhang, Y. Shao, J. Tang, J. Jiang, L. Wang, and S. Li, "Supramolecular asymmetric catalysis mediated by crown ethers and related recognition systems," Green Synthesis and Catalysis, vol. 2, 2021.

[162] E. S. M. El-Sayed, K. Su, P. He, and D Yuan, "Ratiometric fluorescence detection of tetracycline antibiotic based on a polynuclear lanthanide metal organic framework," Sensors and Actuators B: Chemical, vol. 330, Article ID 129314, 2021.

[163] C. J. Murphy, "Sustainability as an emerging design criterion in nanoparticle synthesis and applications," Journal of Materials Chemistry, vol. 18, no. 19, p. 2173, 2008.

[164] S. V. Jadhav, K. M. Jinka, and H. C. Bajaj, "Nanosized sulfated zinc ferrite as catalyst for the synthesis of nopol and other fine chemicals," Catalysis Today, vol. 198, no. 1, pp. 98-105, 2012.

[165] D. Yadav, K. Sandeep, D. Pandey, and R. K. Dutta, "Liposomes for drug delivery," Journal of Biotechnology \& Biomaterials, vol. 7, no. 4, 2017.

[166] J. Li, X. Wang, T. Zhang et al., "A review on phospholipids and their main applications in drug delivery systems," Asian Journal of Pharmaceutical Sciences, vol. 10, no. 2, pp. 81-98, 2015.

[167] R. Ghosh Chaudhuri and S. Paria, "Core/shell nanoparticles: classes, properties, synthesis mechanisms, characterization, and applications," Chemical Reviews, vol. 112, no. 4, pp. 2373-2433, 2011.

[168] C. Caddeo, K. Teskač, C. Sinico, and J. Kristl, "Effect of resveratrol incorporated in liposomes on proliferation and UV-B protection of cells," International Journal of Pharmaceutics, vol. 363, no. 1-2, pp. 183-191, 2008.

[169] A. Omri, Z. E. Suntres, and P. N. Shek, "Enhanced activity of liposomal polymyxin B against Pseudomonas aeruginosa in a rat model of lung infection," Biochemical pharmacology, vol. 64, no. 9, pp. 1407-1413, 2002.

[170] P. K. Angra, C. Oettinger, S. Balakrishna Pai, and M. J. D’Souza, “Amphotericin B microspheres: A therapeutic approach to minimize toxicity while maintaining antifungal efficacy," Journal of Microencapsulation, vol. 26, no. 7, pp. 580-587, 2009

[171] X.-B. Xiong, Y. Huang, W.-l. Lu, H. Zhang, X. Zhang, and Q. Zhang, "Enhanced intracellular uptake of sterically stabilized liposomal doxorubicin in vitro resulting in improved antitumor activity in vivo," Pharmaceutical Research, vol. 22, no. 6, pp. 933-939, 2005.

[172] A. Cabanes, F. Reig, J. M. Garcia-Anton, and M. Arboix, "Evaluation of free and liposome-encapsulated gentamycin for intramuscular sustained release in rabbits," Research in Veterinary Science, vol. 64, no. 3, pp. 213-217, 1998.
[173] H. Xing, K. Hwang, and Y. Lu, "Recent developments of liposomes as nanocarriers for theranostic applications," Theranostics, vol. 6, no. 9, pp. 1336-1352, 2016.

[174] E. Blanco, C. W. Kessinger, B. D. Sumer, and J. Gao, "Multifunctional micellar nanomedicine for cancer therapy," Experimental Biology and Medicine, vol. 234, no. 2, pp. 123-131, 2009.

[175] S. Shirsand, K. Kanani, D. Keerthy, D. Nagendrakumar, and M. Para, "Formulation and evaluation of ketoconazole niosomal gel drug delivery system," International Journal of Pharmaceutical Investigation, vol. 2, no. 4, p. 201, 2012.

[176] A. A. W. Elzainy, X. Gu, F. E. R. Simons, and K. J. Simons, "Hydroxyzine from topical phospholipid liposomal formulations: evaluation of peripheral antihistaminic activity and systemic absorption in a rabbit model," AAPS PharmSci, vol. 5, no. 4, pp. 41-48, 2003.

[177] L. Sercombe, T. Veerati, F. Moheimani, S. Y. Wu, A. K. Sood, and S. Hua, "Advances and challenges of liposome assisted drug delivery," Frontiers in Pharmacology, vol. 6, p. 286, 2015.

[178] M. Alavi, N. Karimi, and M. Safaei, "Application of various types of liposomes in drug delivery systems," Advanced Pharmaceutical Bulletin, vol. 7, no. 1, pp. 3-9, 2017.

[179] A. Dwivedi, A. Mazumder, L. du Plessis, J. L. du Preez, R. K. Haynes, and J. du Plessis, "In vitro anti-cancer effects of artemisone nano-vesicular formulations on melanoma cells," Nanomedicine: Nanotechnology, Biology and Medicine, vol. 11, no. 8, pp. 2041-2050, 2015.

[180] D. Paolino, D. Cosco, R. Muzzalupo, E. Trapasso, N. Picci, and M. Fresta, "Innovative bola-surfactant niosomes as topical delivery systems of 5-fluorouracil for the treatment of skin cancer," International Journal of Pharmaceutics, vol. 353, no. 1-2, pp. 233-42, 2008.

[181] R. P. Gude, M. G. Jadhav, S. G. A. Rao, and A. G. Jagtap, "Effects of niosomal cisplatin and combination of the same with theophylline and with activated macrophages in murine B16F10 melanoma model," Cancer Biotherapy and Radiopharmaceuticals, vol. 17, no. 2, pp. 183-192, 2002.

[182] D. Kerr, A. Rogerson, G. Morrison, A. Florence, and S. Kaye, "Antitumour activity and pharmacokinetics of niosome encapsulated adriamycin in monolayer, spheroid and xenograft," British Journal of Cancer, vol. 58, no. 4, pp. 432-436, 1988.

[183] S. Y. Gaikwad, A. G. Jagtap, A. D. Ingle, S. G. A. Ra, and R. P. Gude, "Antimetastatic efficacy of niosomal pentoxifylline and its combination with activated macrophages in murine B16F10 melanoma model," Cancer Biotherapy and Radiopharmaceuticals, vol. 15, no. 6, pp. 605-615, 2000.

[184] P. Mpungose, Z. Vundla, G. Maguire, and H. Friedrich, "The current status of heterogeneous palladium catalysed heck and Suzuki cross-coupling reactions," Molecules, vol. 23, no. 7, p. 1676, 2018.

[185] A. H. Labulo, B. S. Martincigh, B. Omondi, and V. O. Nyamori, "Advances in carbon nanotubes as efficacious supports for palladium-catalysed carbon-carbon crosscoupling reactions," Journal of Materials Science, vol. 52, no. 16, pp. 9225-9248, 2017

[186] M. Pagliaro, V. Pandarus, R. Ciriminna, F. Béland, and P. Demma Carà, "Heterogeneous versus homogeneous palladium catalysts for cross-coupling reactions," ChemCatChem, vol. 4, no. 4, pp. 432-445, 2012.

[187] M. Gruber, S. Chouzier, K. Koehler, and L. Djakovitch, "Palladium on activated carbon: a valuable heterogeneous 
catalyst for one-pot multi-step synthesis," Applied Catalysis A: General, vol. 265, no. 2, pp. 161-169, 2004.

[188] H. Brunner, N. L. C. de Courcy, and J. P. Genêt, "Heck reactions using aryldiazonium salts towards phosphonic derivatives," Synlett, vol. 2000, no. 2, pp. 201-204, 2000.

[189] R. H. Taylor and F.-X. Felpin, "Suzuki-Miyaura reactions of arenediazonium salts catalyzed by $\mathrm{Pd}(0) / \mathrm{C}$. One-pot chemoselective double cross-coupling reactions," Organic letters, vol. 9, no. 15, pp. 2911-2914, 2007.

[190] F.-X. Felpin and E. Fouquet, "Efficient and practical crosscoupling of arenediazonium tetrafluoroborate salts with boronic acids catalyzed by palladium(0)/barium carbonate," Advanced Synthesis \& Catalysis, vol. 350, no. 6, pp. 863-868, 2008.

[191] F.-X. Felpin, O. Ibarguren, L. Nassar-Hardy, and E. Fouquet, "Synthesis of oxindoles by tandem Heck-reduction-cyclization (HRC) from a single bifunctional, in situ generated $\mathrm{Pd} / \mathrm{C}$ catalyst," The Journal of Organic Chemistry, vol. 74, no. 3, pp. 1349-1352, 2009.

[192] O. Ibarguren, C. Zakri, E. Fouquet, and F.-X. Felpin, "Heterogeneous palladium multi-task catalyst for sequential Heck-reduction-cyclization (HRC) reactions: influence of the support," Tetrahedron Letters, vol. 50, no. 36, pp. 5071-5074, 2009.

[193] Y. Fujii, H. Furugaki, E. Tamura, S. Yano, and K. Kita, "A convenient catalytic method for the synthesis of ethers from alcohols and carbonyl compounds," Bulletin of the Chemical Society of Japan, vol. 78, no. 3, pp. 456-463, 2005.

[194] O. Roy, M. Diekmann, A. Riahi, F. Hénin, and J. Muzart, "Access to optically active linear ketones by one-pot catalytic deprotection, decarboxylation, asymmetric tautomerization from racemic benzyl $\beta$-ketoesters," Chemical Communications, no. 6, pp. 533-534, 2001.

[195] M. S. Kwon, S. Kim, S. Park, W. Bosco, R. K. Chidrala, and J. Park, "One-pot synthesis of imines and secondary amines by Pd-catalyzed coupling of benzyl alcohols and primary amines," The Journal of Organic Chemistry, vol. 74, no. 7, pp. 2877-2879, 2009.

[196] H.-B. Yu, Q.-S. Hu, and L. Pu, "The first optically active BINOL-BINAP copolymer catalyst: highly stereoselective tandem asymmetric reactions," Journal of the American Chemical Society, vol. 122, no. 27, pp. 6500-6501, 2000.

[197] R. S. Robinson and R. J. Taylor, "Quinoxaline synthesis from $\alpha$-hydroxy ketones via a tandem oxidation process using catalysed aerobic oxidation," Synlett, vol. 2005, no. 6, pp. 1003-1005, 2005.

[198] S. Sithambaram, R. Kumar, Y. Son, and S. Suib, "Tandem catalysis: direct catalytic synthesis of imines from alcohols using manganese octahedral molecular sieves," Journal of Catalysis, vol. 253, no. 2, pp. 269-277, 2008.

[199] N. Ravasio, F. Zaccheria, M. Guidotti, and R. Psaro, "Monoand bifunctional heterogeneous catalytic transformation of terpenes and terpenoids," Topics in Catalysis, vol. 27, no. 1, pp. 157-168, 2004.

[200] L. Veum, S. R. Pereira, J. C. van der Waal, and U. Hanefeld, "Catalytic hydrogenation of cyanohydrin esters as a novel approach to $\mathrm{N}$-acylated $\beta$-amino alcohols-reaction optimisation by a design of experiment approach," European Journal of Organic Chemistry, vol. 2006, 2006.

[201] A. R. Mouat, C. L. Whitford, B.-R. Chen et al., "Synthesis of Supported Pd0 nanoparticles from a single-site Pd2+ surface complex by alkene reduction," Chemistry of Materials, vol. 30, no. 3, pp. 1032-1044, 2018.
[202] S. N. Jadhav, A. S. Kumbhar, C. V. Rode, and R. S. Salunkhe, "Ligand-free Pd catalyzed cross-coupling reactions in an aqueous hydrotropic medium," Green Chemistry, vol. 18, no. 7, pp. 1898-1911, 2016.

[203] H. U. Blaser, B. Pugin, F. Spindler, and B. Cornils, Applied Homogeneous Catalysis with Organometallic Compounds, Wiley, Hoboken, NJ, USA, 2000.

[204] M. E. Davis, Aqueous-Phase Organometallic Catalysis: Concepts and Applications, Wiley-VCH, Weinheim, Germany, 1998.

[205] B. M. Bhanage, S.-i. Fujita, T. Yoshida, Y. Sano, and M. Arai, "Simultaneous application of two or more supported liquidphase organometallic catalysts: heterogeneous multifunctional reaction systems," Tetrahedron Letters, vol. 44, no. 17, pp. 3505-3507, 2003.

[206] A. Molnár, "Efficient, selective, and recyclable palladium catalysts in carbon-carbon coupling reactions," Chemical Reviews, vol. 111, no. 3, pp. 2251-2320, 2011.

[207] M. Lamblin, L. Nassar-Hardy, J.-C. Hierso, E. Fouquet, and F.-X. Felpin, "Recyclable heterogeneous palladium catalysts in pure water: sustainable developments in Suzuki, Heck, Sonogashira and Tsuji-Trost reactions," Advanced Synthesis \& Catalysis, vol. 352, no. 1, pp. 33-79, 2010.

[208] E. Auer, A. Freund, J. Pietsch, and T. Tacke, "Carbons as supports for industrial precious metal catalysts," Applied Catalysis A: General, vol. 173, no. 2, pp. 259-271, 1998.

[209] D. B. Mawhinney, V. Naumenko, A. Kuznetsova, J. T. Yates, J. Liu, and R. E. Smalley, "Infrared spectral evidence for the etching of carbon nanotubes: ozone oxidation at $298 \mathrm{~K}$," Journal of the American Chemical Society, vol. 122, no. 10, pp. 2383-2384, 2000.

[210] Z. Qian, J. Ma, J. Zhou et al., "Facile synthesis of halogenated multi-walled carbon nanotubes and their unusual photoluminescence," Journal of Materials Chemistry, vol. 22, no. 41, Article ID 22113, 2012.

[211] U. Dettlaff-Weglikowska, V. Skákalová, R. Graupner et al., "Effect of $\mathrm{SOCl}_{2}$ treatment on electrical and mechanical properties of single-wall carbon nanotube networks," Journal of the American Chemical Society, vol. 127, no. 14, pp. 5125-5131, 2005.

[212] J. Chen, H. Liu, W. A. Weimer, M. D. Halls, D. H. Waldeck, and G. C. Walker, "Noncovalent engineering of carbon nanotube surfaces by rigid, functional conjugated polymers," Journal of the American Chemical Society, vol. 124, no. 31, pp. 9034-9035, 2002.

[213] H. Yoon, S. Ko, and J. Jang, "Nitrogen-doped magnetic carbon nanoparticles as catalyst supports for efficient recovery and recycling," Chemical Communications, vol. 14, no. 14, pp. 1468-1470, 2007.

[214] S. Rana, S. Maddila, K. Yalagala, S. Maddila, and S. B. Jonnalagadda, "Covalent modification of organofunctionalized graphene oxide and its scope as catalyst for one-pot pyrazolo-pyranopyrimidine derivatives," ChemistryOpen, vol. 4, no. 6, pp. 703-707, 2015.

[215] B. Lakshminarayana, L. Mahendar, P. Ghosal, G. Satyanarayana, and C. Subrahmanyam, "Nano-sized recyclable PdO supported carbon nanostructures for Heck reaction: influence of carbon materials," ChemistrySelect, vol. 2, no. 9, pp. 2700-2707, 2017.

[216] M. L. Kantam, R. Chakravarti, V. R. Chintareddy, B. Sreedhar, and S. Bhargava, "Palladium-catalyzed heck coupling-hydrogenation: highly efficient one-pot synthesis of dibenzyls and alkyl phenyl esters," Advanced Synthesis \& Catalysis, vol. 350, no. 16, pp. 2544-2550, 2008. 
[217] B. D. Adams and A. Chen, "The role of palladium in a hydrogen economy," Materials Today, vol. 14, no. 6, pp. 282-289, 2011.

[218] V. L. Budarin, P. S. Shuttleworth, J. H. Clark, and R. Luque, "Industrial applications of C-C coupling reactions," Current Organic Synthesis, vol. 7, no. 6, pp. 614-627, 2010.

[219] T. Kamal, "Aminophenols formation from nitrophenols using agar biopolymer hydrogel supported $\mathrm{CuO}$ nanoparticles catalyst," Polymer Testing, vol. 77, Article ID 105896, 2019.

[220] T. Ishida and M. Haruta, "N-formylation of amines via the aerobic oxidation of methanol over supported gold nanoparticles," ChemSusChem, vol. 2, no. 6, pp. 538-541, 2009.

[221] L. L. Santos, P. Serna, and A. Corma, "Chemoselective synthesis of substituted imines, secondary amines, and $\beta$-amino carbonyl compounds from nitroaromatics through cascade reactions on gold catalysts," Chemistry - A European Journal, vol. 15, no. 33, pp. 8196-8203, 2009.

[222] H. Sun, F.-Z. Su, J. Ni, Y. Cao, H.-Y. He, and K.-N. Fan, "Gold supported on hydroxyapatite as a versatile multifunctional catalyst for the direct tandem synthesis of imines and oximes," Angewandte Chemie International Edition, vol. 48, no. 24, pp. 4390-4393, 2009.

[223] A. Grirrane, A. Corma, and H. Garcia, "Highly active and selective gold catalysts for the aerobic oxidative condensation of benzylamines to imines and one-pot, two-step synthesis of secondary benzylamines," Journal of Catalysis, vol. 264, no. 2, pp. 138-144, 2009.

[224] M. Ventura, A. Marinas, and M. E. Domine, "Catalytic processes for biomass-derived platform molecules valorisation," Topics in Catalysis, vol. 63, no. 9, pp. 846-865, 2020.

[225] B. Zhu, M. Lazar, B. G. Trewyn, and R. J. Angelici, “Aerobic oxidation of amines to imines catalyzed by bulk gold powder and by alumina-supported gold," Journal of Catalysis, vol. 260, no. 1, pp. 1-6, 2008.

[226] G. Budroni and A. Corma, "Gold and gold-platinum as active and selective catalyst for biomass conversion: synthesis of $\gamma$-butyrolactone and one-pot synthesis of pyrrolidone," Journal of Catalysis, vol. 257, no. 2, pp. 403-408, 2008.

[227] M. Pristovsek, M. Zorn, and M. Weyers, "In situ study of GaAs growth mechanisms using tri-methyl gallium and triethyl gallium precursors in metal-organic vapour phase epitaxy," Journal of Crystal growth, vol. 262, no. 1-4, pp. 78-83, 2004.

[228] R. Juárez, P. Concepción, A. Corma, V. Fornés, and H. García, "Gold-catalyzed phosgene-free synthesis of polyurethane precursors," Angewandte Chemie International Edition, vol. 49, no. 7, pp. 1286-1290, 2010.

[229] C. Xu, S. De, A. M. Balu, M. Ojeda, and R. Luque, "Mechanochemical synthesis of advanced nanomaterials for catalytic applications," Chemical Communications, vol. 51, no. 31, pp. 6698-6713, 2015.

[230] M. Zhou, C. Chen, P. Liu et al., "Catalytic hydrotreatment of $\beta$-O-4 ether in lignin: cleavage of the $\mathrm{C}-\mathrm{O}$ bond and hydrodeoxygenation of lignin-derived phenols in one pot," ACS Sustainable Chemistry \& Engineering, vol. 8, no. 38, pp. 14511-14523, 2020.

[231] B. Zümreoglu-Karan and A. Ay, "Layered double hydroxides-multifunctional nanomaterials," Chemical Papers, vol. 66, no. 1, pp. 1-10, 2012.

[232] H. R. Mahmoud, "Highly dispersed $\mathrm{Cr}_{2} \mathrm{O}_{3}-\mathrm{ZrO}_{2}$ binary oxide nanomaterials as novel catalysts for ethanol conversion," Journal of Molecular Catalysis A: Chemical, vol. 392, pp. 216-222, 2014.
[233] R. K. Sharma, S. Sharma, S. Dutta, R. Zboril, and M. B. Gawande, "Silica-nanosphere-based organic-inorganic hybrid nanomaterials: synthesis, functionalization and applications in catalysis," Green Chemistry, vol. 17, no. 6, pp. 3207-3230, 2015.

[234] M. Khalil, G. T. Kadja, and M. M. Ilmi, “Advanced nanomaterials for catalysis: current progress in fine chemical synthesis, hydrocarbon processing, and renewable energy," Journal of Industrial and Engineering Chemistry, vol. 93, 2020.

[235] M. I. Qadir, J. D. Scholten, and J. Dupont, " $\mathrm{TiO}_{2}$ nanomaterials: highly active catalysts for the oxidation of hydrocarbons," Journal of Molecular Catalysis A: Chemical, vol. 383-384, pp. 225-230, 2014.

[236] X. Cui, A. P. van Muyden, and P. J. Dyson, "Frontispiece: utility of core-shell nanomaterials in the catalytic transformations of renewable substrates," Chemistry-A European Journal, vol. 27, no. 1, 2021.

[237] A. Liu and X. Ren, "Power ready for driving catalysis and sensing: nanomaterials designed for renewable energy storage," in Advanced Nanomaterials for Pollutant Sensing and Environmental Catalysis, pp. 307-346, Elsevier, Amsterdam, Netherlands, 2020.

[238] P. Sathishkumar, R. Sweena, J. J. Wu, and S. Anandan, "Synthesis of $\mathrm{CuO}-\mathrm{ZnO}$ nanophotocatalyst for visible light assisted degradation of a textile dye in aqueous solution," Chemical Engineering Journal, vol. 171, no. 1, pp. 136-140, 2011.

[239] S. Chaturvedi, P. N. Dave, and N. K. Shah, "Applications of nano-catalyst in new era," Journal of Saudi Chemical Society, vol. 16, no. 3, pp. 307-325, 2012.

[240] M. Anjum, R. Miandad, M. Waqas, F. Gehany, and M. A. Barakat, "Remediation of wastewater using various nano-materials," Arabian Journal of Chemistry, vol. 12, no. 8, pp. 4897-4919, 2019.

[241] P. Bhanja, A. Modak, and A. Bhaumik, "Supported porous nanomaterials as efficient heterogeneous catalysts for $\mathrm{CO}_{2}$ fixation reactions," Chemistry-A European Journal, vol. 24, no. 29, pp. 7278-7297, 2018.

[242] P. Ríos, A. Rodríguez, and J. López-Serrano, "Mechanistic studies on the selective reduction of $\mathrm{CO}_{2}$ to the aldehyde level by a bis(phosphino)boryl (PBP)-supported nickel complex," ACS Catalysis, vol. 6, no. 9, pp. 5715-5723, 2016.

[243] Y. Kuwahara, Y. Fujie, and H. Yamashita, "Poly(ethyleneimine)-tethered Ir complex catalyst immobilized in titanate nanotubes for hydrogenation of $\mathrm{CO}_{2}$ to formic acid," ChemCatChem, vol. 9, no. 11, pp. 1906-1914, 2017.

[244] O. Martin and J. Pérez-Ramírez, "New and revisited insights into the promotion of methanol synthesis catalysts by $\mathrm{CO}_{2}$," Catalysis Science \& Technology, vol. 3, no. 12, pp. 3343-3352, 2013.

[245] K. Takeishi, "Dimethyl ether and catalyst development for production from syngas," Biofuels, vol. 1, no. 1, pp. 217-226, 2010.

[246] D. M. Brown, B. L. Bhatt, T. H. Hsiung, J. J. Lewnard, and F. J. Waller, "Novel technology for the synthesis of dimethyl ether from syngas," Catalysis Today, vol. 8, no. 3, pp. 279-304, 1991.

[247] M. A. A. Aziz, A. A. Jalil, S. Triwahyono, R. R. Mukti, Y. H. Taufiq-Yap, and M. R. Sazegar, "Highly active Nipromoted mesostructured silica nanoparticles for $\mathrm{CO}_{2}$ methanation," Applied Catalysis B: Environmental, vol. 147, pp. 359-368, 2014. 
[248] G. Zhou, T. Wu, H. Xie, and X. Zheng, "Effects of structure on the carbon dioxide methanation performance of Cobased catalysts," International Journal of Hydrogen Energy, vol. 38, no. 24, pp. 10012-10018, 2013.

[249] B. Karimi, F. Mansouri, and H. Vali, "A highly water-dispersible/magnetically separable palladium catalyst: selective transfer hydrogenation or direct reductive $\mathrm{N}$-formylation of nitroarenes in water," ChemPlusChem, vol. 80, no. 12, pp. 1750-1759, 2015.

[250] X.-F. Liu, C. Qiao, X.-Y. Li, and L.-N. He, "Carboxylatepromoted reductive functionalization of $\mathrm{CO}_{2}$ with amines and hydrosilanes under mild conditions," Green Chemistry, vol. 19, no. 7, pp. 1726-1731, 2017.

[251] F. Bastan, M. Kazemeini, A. Larimi, and H. Maleki, "Production of renewable hydrogen through aqueous-phase reforming of glycerol over $\mathrm{Ni} / \mathrm{Al}_{2} \mathrm{O}_{3} \mathrm{MgO}$ nano-catalyst," International Journal of Hydrogen Energy, vol. 43, no. 2, pp. 614-621, 2018.

[252] P. A. Berseth, A. G. Harter, R. Zidan et al., "Carbon nanomaterials as catalysts for hydrogen uptake and release in $\mathrm{NaAlH}_{4}$," Nano Letters, vol. 9, no. 4, pp. 1501-1505, 2009.

[253] M. Hu, Z. Yao, and X. Wang, "Graphene-based nanomaterials for catalysis," Industrial \& Engineering Chemistry Research, vol. 56, no. 13, pp. 3477-3502, 2017.

[254] A. Tiwari, A. Bhatia, U. Chadha, S. Kandregula, and S. K. Selvaraj, "Nanomaterials for electromagnetic interference shielding applications: a review," Nano, Article ID 2230001, 2022.

[255] M. Yoshida, K. Takanabe, K. Maeda et al., "Role and function of noble-metal/Cr-layer core/shell structure cocatalysts for photocatalytic overall water splitting studied by model electrodes," The Journal of Physical Chemistry C, vol. 113, no. 23, pp. 10151-10157, 2009.

[256] Z. Wu, X. Wang, J. Huang, and F. Gao, "A Co-doped Ni-Fe mixed oxide mesoporous nanosheet array with low overpotential and high stability towards overall water splitting," Journal of Materials Chemistry A, vol. 6, no. 1, pp. 167-178, 2018.

[257] J. Soldat, G. W. Busser, M. Muhler, and M. Wark, " $\mathrm{Cr}_{2} \mathrm{O}_{3}$ nanoparticles on $\mathrm{Ba}_{5} \mathrm{Ta}_{4} \mathrm{O}_{15}$ as a noble-metal-free oxygen evolution co-catalyst for photocatalytic overall water splitting," ChemCatChem, vol. 8, no. 1, pp. 153-156, 2016.

[258] T. Zhao, J. Gao, J. Wu, P. He, Y. Li, and J. Yao, "Highly active cobalt/tungsten carbide@N-doped porous carbon nanomaterials derived from metal-organic frameworks as bifunctional catalysts for overall water splitting," Energy Technology, vol. 7, 2019.

[259] H. Yi, D. Huang, L. Qin et al., "Selective prepared carbon nanomaterials for advanced photocatalytic application in environmental pollutant treatment and hydrogen production," Applied Catalysis B: Environmental, vol. 239, pp. 408-424, 2018.

[260] Y. Yang, M. Wu, X. Zhu et al., "2020 roadmap on two-dimensional nanomaterials for environmental catalysis," Chinese Chemical Letters, vol. 30, 2019.

[261] S. Li, Z. Zhao, J. Zhao, Z. Zhang, X. Li, and J. Zhang, "Recent advances of ferro-, piezo-, and pyroelectric nanomaterials for catalytic applications," ACS Applied Nano Materials, vol. 3, no. 2, pp. 1063-1079, 2020.

[262] H. Jin, C. Guo, X. Liu et al., "Emerging two-dimensional nanomaterials for electrocatalysis," Chemical Reviews, vol. 118, no. 13, pp. 6337-6408, 2018.

[263] S. Vijaikumar and K. Pitchumani, "Efficient microwave activation of hydrotalcite clays in Michael addition under solvent-free conditions," 2010, http://hdl.handle.net/ $123456789 / 7927$.

[264] U. R. Pillai, E. Sahle-Demessie, and R. S. Varma, "Ultrasound-assisted epoxidation of olefins and $\alpha, \beta$-unsaturated ketones over hydrotalcites using hydrogen peroxide," Synthetic Communications, vol. 33, no. 12, pp. 2017-2027, 2003.

[265] A. Biffis, M. Zecca, and M. Basato, "Metallic palladium in the heck reaction: active catalyst or convenient precursor?" European Journal of Inorganic Chemistry, vol. 2001, no. 5, pp. 1131-1133, 2001.

[266] Y. Li, X. Zheng, and Q. Chu, "Bio-based nanomaterials for cancer therapy," Nano Today, vol. 38, Article ID 101134, 2021.

[267] M. L. Steigerwald and L. E. Brus, "Semiconductor crystallites: a class of large molecules," Accounts of Chemical Research, vol. 23, no. 6, pp. 183-188, 1990.

[268] Z. A. Peng and X. Peng, "Formation of high-quality CdTe, $\mathrm{CdSe}$, and CdS nanocrystals using CdO as precursor," Journal of the American Chemical Society, vol. 123, no. 1, pp. 183-184, 2001.

[269] L. Qu, Z. A. Peng, and X. Peng, "Alternative routes toward high quality CdSe nanocrystals," Nano Letters, vol. 1, no. 6, pp. 333-337, 2001.

[270] S. Al-thabaiti, M. Malik, A. Al-youbi, Z. Khan, and J. I. Hussain, "Effects of surfactant and polymer on the morphology of advanced nanomaterials in aqueous solution," International Journal of Electrochemical Science, vol. 8, pp. 204-218, 2013.

[271] S. Kanaoka, N. Yagi, Y. Fukuyama et al., "Thermosensitive gold nanoclusters stabilized by well-defined vinyl ether star polymers: reusable and durable catalysts for aerobic alcohol oxidation," Journal of the American Chemical Society, vol. 129, no. 40, pp. 12060-12061, 2007

[272] M. D. Hughes, Y.-J. Xu, P. Jenkins et al., "Tunable gold catalysts for selective hydrocarbon oxidation under mild conditions," Nature, vol. 437, no. 7062, pp. 1132-1135, 2005.

[273] J. A. Dahl, B. L. S. Maddux, and J. E. Hutchison, "Toward greener nanosynthesis," Chemical Reviews, vol. 107, no. 6, pp. 2228-2269, 2007.

[274] P. Raveendran, J. Fu, and S. L. Wallen, "Completely "green" synthesis and stabilization of metal nanoparticles," Journal of the American Chemical Society, vol. 125, no. 46, pp. 13940-13941, 2003.

[275] S. S. Shankar, A. Rai, B. Ankamwar, A. Singh, A. Ahmad, and M. Sastry, "Biological synthesis of triangular gold nanoprisms," Nature Materials, vol. 3, no. 7, pp. 482-488, 2004.

[276] S. S. Shankar, A. Rai, A. Ahmad, and M. Sastry, "Controlling the optical properties of lemongrass extract synthesized gold nanotriangles and potential application in infrared-absorbing optical coatings," Chemistry of Materials, vol. 17, no. 3, pp. 566-572, 2005.

[277] B. Liu, J. Xie, J. Y. Lee, Y. P. Ting, and J. P. Chen, "Optimization of high-yield biological synthesis of single-crystalline gold nanoplates," The Journal of Physical Chemistry B, vol. 109, no. 32, pp. 15256-15263, 2005.

[278] R. Brayner, M.-J. Vaulay, F. Fiévet, and T. Coradin, “Alginate-mediated growth of $\mathrm{Co}, \mathrm{Ni}$, and $\mathrm{CoNi}$ nanoparticles: influence of the biopolymer structure," Chemistry of $\mathrm{Ma}$ terials, vol. 19, no. 5, pp. 1190-1198, 2007.

[279] N. Vigneshwaran, A. A. Kathe, P. V. Varadarajan, R. P. Nachane, and R. H. Balasubramanya, "Silver-protein (core-shell) nanoparticle production using spent 
mushroom substrate," Langmuir, vol. 23, no. 13, pp. 7113-7117, 2007.

[280] F. Liu, L. Wang, Q. Sun, L. Zhu, X. Meng, and F.-S. Xiao, "Transesterification catalyzed by ionic liquids on superhydrophobic mesoporous polymers: heterogeneous catalysts that are faster than homogeneous catalysts," Journal of the American Chemical Society, vol. 134, no. 41, pp. 16948-16950, 2012.

[281] P. A. Zapata, J. Faria, M. P. Ruiz, R. E. Jentoft, and D. E. Resasco, "Hydrophobic zeolites for biofuel upgrading reactions at the liquid-liquid interface in water/oil emulsions," Journal of the American Chemical Society, vol. 134, no. 20, pp. 8570-8578, 2012.

[282] E. Rafiee and S. Shahebrahimi, "Nano silica with high surface area from rice husk as a support for 12-tungstophosphoric acid: an efficient nano catalyst in some organic reactions," Chinese Journal of Catalysis, vol. 33, no. 7-8, pp. 1326-1333, 2012.

[283] Y. Tao, H. Kanoh, L. Abrams, and K. Kaneko, "Mesoporemodified zeolites: preparation, characterization, and applications," Chemical Reviews, vol. 106, no. 3, pp. 896-910, 2006.

[284] X. Meng and F.-S. Xiao, "Green routes for synthesis of zeolites," Chemical Reviews, vol. 114, no. 2, pp. 1521-1543, 2014.

[285] K. Möller and T. Bein, "Mesoporosity-a new dimension for zeolites," Chemical Society Reviews, vol. 42, no. 9, pp. 3689-3707, 2013.

[286] D. P. Serrano, J. M. Escola, and P. Pizarro, "Synthesis strategies in the search for hierarchical zeolites," Chemical Society Reviews, vol. 42, no. 9, pp. 4004-4035, 2013.

[287] J. A. Melero, R. van Grieken, and G. Morales, "Advances in the synthesis and catalytic applications of organosulfonicfunctionalized mesostructured materials," Chemical Reviews, vol. 106, no. 9, pp. 3790-3812, 2006.

[288] D. Margolese, J. A. Melero, S. C. Christiansen, B. F. Chmelka, and G. D. Stucky, "Direct syntheses of ordered SBA-15 mesoporous silica containing sulfonic acid groups," Chemistry of Materials, vol. 12, no. 8, pp. 2448-2459, 2000.

[289] X. Wang, R. Liu, M. M. Waje et al., "Sulfonated ordered mesoporous carbon as a stable and highly active protonic acid catalyst," Chemistry of Materials, vol. 19, no. 10, pp. 2395-2397, 2007.

[290] R. Xing, Y. M. Liu, Y. Wang et al., “Active solid acid catalysts prepared by sulfonation of carbonization-controlled mesoporous carbon materials," Microporous Mesoporous Materials, vol. 105, pp. 41-48, 2007.

[291] M. Toda, A. Takagaki, M. Okamura et al., "Biodiesel made with sugar catalyst," Nature, vol. 438, no. 7065, p. 178, 2005.

[292] S. Suganuma, K. Nakajima, M. Kitano et al., "Hydrolysis of cellulose by amorphous carbon bearing $\mathrm{SO}_{3} \mathrm{H}, \mathrm{COOH}$, and $\mathrm{OH}$ groups," Journal of the American Chemical Society, vol. 130, no. 38, pp. 12787-12793, 2008.

[293] W. Wang, X. Zhuang, Q. Zhao, and Y. Wan, "Self-assembly synthesis of a high-content sulfonic acid group functionalized ordered mesoporous polymer-based solid as a stable and highly active acid catalyst," Journal of Materials Chemistry, vol. 22, no. 31, Article ID 15874, 2012.

[294] L. Wang, H. Wang, F. Liu et al., "Selective catalytic production of 5-hydroxymethylfurfural from glucose by adjusting catalyst wettability," ChemSusChem, vol. 7, no. 2, pp. 402-406, 2014.

[295] B. Zhao, M. Chen, Q. Guo, and Y. Fu, "Electrocatalytic hydrogenation of furfural to furfuryl alcohol using platinum supported on activated carbon fibers," Electrochimica Acta, vol. 135, pp. 139-146, 2014.

[296] M. M. Villaverde, T. F. Garetto, and A. J. Marchi, "Liquidphase transfer hydrogenation of furfural to furfuryl alcohol on $\mathrm{Cu}-\mathrm{Mg}-\mathrm{Al}$ catalysts," Catalysis Communications, vol. 58, pp. 6-10, 2015.

[297] D. Vargas-Hernández, J. M. Rubio-Caballero, J. SantamaríaGonzález et al., "Furfuryl alcohol from furfural hydrogenation over copper supported on SBA-15 silica catalysts," Journal of Molecular Catalysis A: Chemical, vol. 383-384, pp. 106-113, 2014.

[298] Q. Yuan, D. Zhang, L. v. Haandel et al., "Selective liquid phase hydrogenation of furfural to furfuryl alcohol by $\mathrm{Ru} / \mathrm{Zr}$ MOFs," Journal of Molecular Catalysis A: Chemical, vol. 406, pp. 58-64, 2015.

[299] B. Chen, F. Li, Z. Huang, and G. Yuan, "Tuning catalytic selectivity of liquid-phase hydrogenation of furfural via synergistic effects of supported bimetallic catalysts," Applied Catalysis A: General, vol. 500, pp. 23-29, 2015.

[300] M. Audemar, C. Ciotonea, K. De Oliveira Vigier et al., "Selective hydrogenation of furfural to furfuryl alcohol in the presence of a recyclable cobalt/SBA-15 catalyst," ChemSusChem, vol. 8, no. 11, pp. 1885-1891, 2015.

[301] M. J. Taylor, L. J. Durndell, M. A. Isaacs et al., "Highly selective hydrogenation of furfural over supported $\mathrm{Pt}$ nanoparticles under mild conditions," Applied Catalysis B: Environmental, vol. 180, pp. 580-585, 2016.

[302] G. Y. Jeong, A. K. Singh, S. Sharma, K. W. Gyak, R. A. Maurya, and D. P. Kim, "One-flow syntheses of diverse heterocyclic furan chemicals directly from fructose via tandem transformation platform," NPG Asia Materials, vol. 7, pp. 1-8, 2015.

[303] T. Mitsudome, A. Noujima, T. Mizugaki, K. Jitsukawa, and K. Kaneda, "Supported gold nanoparticles as a reusable catalyst for synthesis of lactones from diols using molecular oxygen as an oxidant under mild conditions," Green Chemistry, vol. 11, no. 6, pp. 793-797, 2009.

[304] Q. Hou, B. Zheng, C. Bi et al., "Liquid-phase cascade acylation/dehydration over various zeolite catalysts to synthesize 2-methylanthraquinone through an efficient one-pot strategy," Journal of Catalysis, vol. 268, no. 2, pp. 376-383, 2009.

[305] B. Zong, B. Sun, S. Cheng et al., "Green production technology of the monomer of nylon-6: caprolactam," Engineering, vol. 3, no. 3, pp. 379-384, 2017.

[306] I. Delidovich and R. Palkovits, "Catalytic versus stoichiometric reagents as a key concept for green chemistry," Green Chemistry, vol. 18, no. 3, pp. 590-593, 2016.

[307] V. R. Ruiz, A. Corma, and M. J. Sabater, "New route for the synthesis of benzimidazoles by a one-pot multistep process with mono and bifunctional solid catalysts," Tetrahedron, vol. 66, no. 3, pp. 730-735, 2010.

[308] W. Bonrath and T. Netscher, "Catalytic processes in vitamins synthesis and production," Applied Catalysis A: General, vol. 280, no. 1, pp. 55-73, 2005.

[309] G. Centi and S. Perathoner, "Catalysis: role and challenges for a sustainable energy," Topics in Catalysis, vol. 52, no. 8, pp. 948-961, 2009.

[310] Z. Liu and J. Liang, "Methanol to olefin conversion catalysts," Current Opinion in Solid State and Materials Science, vol. 4, no. 1, pp. 80-84, 1999.

[311] C. Hao, S. Wang, M. Li, L. Kang, and X. Ma, "Hydrogenation of $\mathrm{CO}_{2}$ to formic acid on supported ruthenium catalysts," Catalysis Today, vol. 160, no. 1, pp. 184-190, 2011. 
[312] S. Bera, J. E. Lee, S. B. Rawal, and W. I. Lee, "Size-dependent plasmonic effects of $\mathrm{Au}$ and $\mathrm{Au} @ \mathrm{SiO}_{2}$ nanoparticles in photocatalytic $\mathrm{CO}_{2}$ conversion reaction of $\mathrm{Pt} / \mathrm{TiO}_{2}$," Applied Catalysis B: Environmental, vol. 199, pp. 55-63, 2016.

[313] M. Miyano, H. Zhang, M. Yoshiba, and Y. Izumi, "Selective photoconversion of carbon dioxide into methanol using layered double hydroxides at $0.40 \mathrm{MPa}$," Energy Technology, vol. 5, no. 6, pp. 892-900, 2017.

[314] K. Sordakis, A. Tsurusaki, M. Iguchi, H. Kawanami, Y. Himeda, and G. Laurenczy, "Carbon dioxide to methanol: the aqueous catalytic way at room temperature," Chemistry - A European Journal, vol. 22, no. 44, pp. 15605-15608, 2016.

[315] H.-G. Kim, C.-S. Lim, D.-W. Kim, D.-H. Cho, D.-K. Lee, and J. S. Chung, "Multifunctional alkanolamine as a catalyst for $\mathrm{CO}_{2}$ and propylene oxide cycloaddition," Molecular Catalysis, vol. 438, pp. 121-129, 2017.

[316] X. Feng, J. D. Sloppy, T. J. LaTempa et al., "Synthesis and deposition of ultrafine Pt nanoparticles within high aspect ratio $\mathrm{TiO}_{2}$ nanotube arrays: application to the photocatalytic reduction of carbon dioxide," Journal of Materials Chemistry, vol. 21, no. 35, pp. 13429-13433, 2011.

[317] B. White, M. Yin, A. Hall et al., "Complete CO oxidation over $\mathrm{Cu}_{2} \mathrm{O}$ nanoparticles supported on silica gel," Nano Letters, vol. 6, no. 9, pp. 2095-2098, 2006.

[318] S. Gao, Y. Lin, X. Jiao et al., "Partially oxidized atomic cobalt layers for carbon dioxide electroreduction to liquid fuel," Nature, vol. 529, no. 7584, pp. 68-71, 2016.

[319] R. Reske, H. Mistry, F. Behafarid, B. Roldan Cuenya, and P. Strasser, "Particle size effects in the catalytic electroreduction of $\mathrm{CO}_{2}$ on $\mathrm{Cu}$ nanoparticles," Journal of the American Chemical Society, vol. 136, no. 19, pp. 6978-6986, 2014.

[320] R. L. Cook, R. C. MacDuff, and A. F. Sammells, "Photoelectrochemical carbon dioxide reduction to hydrocarbons at ambient temperature and pressure," Journal of the Electrochemical Society, vol. 135, no. 12, pp. 3069-3070, 1988.

[321] D. Qin, Y. Zhou, W. Wang et al., "Recent advances in twodimensional nanomaterials for photocatalytic reduction of $\mathrm{CO}_{2}$ : insights into performance, theories and perspective," Journal of Materials Chemistry A, vol. 8, no. 37, pp. 19156-19195, 2020.

[322] S. Zhang, P. Kang, S. Ubnoske et al., "Polyethylenimineenhanced electrocatalytic reduction of $\mathrm{CO}_{2}$ to formate at nitrogen-doped carbon nanomaterials," Journal of the American Chemical Society, vol. 136, no. 22, pp. 7845-7848, 2014.

[323] X. Duan, J. Xu, Z. Wei et al., "Metal-free carbon materials for $\mathrm{CO}_{2}$ electrochemical reduction," Advanced Materials, vol. 29, no. 41, Article ID 1701784, 2017.

[324] H. Li, X. Zhou, W. Zhai et al., "Phase engineering of nanomaterials for clean energy and catalytic applications," Advanced Energy Materials, vol. 10, no. 40, Article ID 2002019, 2020.

[325] D. Li, H. Liu, and L. Feng, "A review on advanced FeNibased catalysts for water splitting reaction," Energy \& Fuels, vol. 34, no. 11, pp. 13491-13522, 2020.

[326] J. Joy, J. Mathew, and S. C. George, "Nanomaterials for photoelectrochemical water splitting-review," International Journal of Hydrogen Energy, vol. 43, no. 10, pp. 4804-4817, 2018.

[327] Y. Hou, Z. Wen, S. Cui, S. Ci, S. Mao, and J. Chen, "An advanced nitrogen-doped graphene/cobalt-embedded porous carbon polyhedron hybrid for efficient catalysis of oxygen reduction and water splitting," Advanced Functional Materials, vol. 25, no. 6, pp. 872-882, 2014.

[328] B. K. Ghosh, S. Hazra, B. Naik, and N. N. Ghosh, "Preparation of $\mathrm{Cu}$ nanoparticle loaded SBA-15 and their excellent catalytic activity in reduction of variety of dyes," Powder Technology, vol. 269, pp. 371-378, 2015.

[329] Y. Li, Z. Liu, Y. Wu et al., "Carbon dots- $\mathrm{TiO}_{2}$ nanosheets composites for photoreduction of $\mathrm{Cr}(\mathrm{VI})$ under sunlight illumination: favorable role of carbon dots," Applied Catalysis B: Environmental, vol. 224, pp. 508-517, 2018.

[330] G. L. M. Léonard, S. Remy, and B. Heinrichs, "Doping $\mathrm{TiO}_{2}$ films with carbon nanotubes to simultaneously optimise antistatic, photocatalytic and superhydrophilic properties," Journal of Sol-Gel Science and Technology, vol. 79, no. 3, pp. 413-425, 2016.

[331] J. Wang, L. Tang, G. Zeng et al., “ $0 \mathrm{D} / 2 \mathrm{D}$ interface engineering of carbon quantum dots modified $\mathrm{Bi}_{2} \mathrm{WO}_{6}$ ultrathin nanosheets with enhanced photoactivity for full spectrum light utilization and mechanism insight," Applied Catalysis B: Environmental, vol. 222, pp. 115-123, 2018.

[332] A. Le Goff, V. Artero, B. Jousselme et al., "From hydrogenases to noble metal-free catalytic nanomaterials for $\mathrm{H}_{2}$ production and uptake," Science, vol. 326, no. 5958, pp. 1384-1387, 2009.

[333] M. U. Niemann, S. S. Srinivasan, A. R. Phani, A. Kumar, D. Y. Goswami, and E. K. Stefanakos, "Nanomaterials for hydrogen storage applications: a review," Journal of Nanomaterials, vol. 2008, Article ID 950967, 9 pages, 2008.

[334] H. Shimada, "Support effect on the catalytic activity and properties of sulfided molybdenum catalysts," Journal of Catalysis, vol. 110, no. 2, pp. 275-284, 1988.

[335] M. Imran, A. B. Yousaf, X. Zhou et al., "Pd/TiO nanocatalyst with strong metal-support interaction for highly efficient durable heterogeneous hydrogenation," The Journal of Physical Chemistry C, vol. 121, no. 2, pp. 1162-1170, 2017.

[336] A. A. Shesterkina, L. M. Kustov, A. A. Strekalova, and V. B. Kazansky, "Heterogeneous iron-containing nanocatalysts-promising systems for selective hydrogenation and hydrogenolysis," Catalysis Science \& Technology, vol. 10, no. 10, pp. 3160-3174, 2020.

[337] S. Cai, D. Wang, Z. Niu, and Y. Li, "Progress in organic reactions catalyzed by bimetallic nanomaterials," Chinese Journal of Catalysis, vol. 34, no. 11, pp. 1964-1974, 2013.

[338] B. C. Hodges, E. L. Cates, and J.-H. Kim, "Challenges and prospects of advanced oxidation water treatment processes using catalytic nanomaterials," Nature Nanotechnology, vol. 13, no. 8, pp. 642-650, 2018.

[339] L. L. Chng, J. Zhang, J. Yang, M. Amoura, and J. Y. Ying, "C$\mathrm{C}$ bond formation via $\mathrm{C}-\mathrm{H}$ activation and $\mathrm{C}-\mathrm{N}$ bond formation via oxidative amination catalyzed by palladiumpolyoxometalate nanomaterials using dioxygen as the terminal oxidant," Advanced Synthesis \& Catalysis, vol. 353, no. 16, pp. 2988-2998, 2011.

[340] Z. Fang, Y. Wang, C. Liu et al., "Rational design of metal nanoframes for catalysis and plasmonics," Small, vol. 11, no. 22, pp. 2593-2605, 2015.

[341] H. Jiang, Q. He, Y. Zhang, and L. Song, "Structural selfreconstruction of catalysts in electrocatalysis," Accounts of Chemical Research, vol. 51, 2018.

[342] D. P. Cormode, L. Gao, and H. Koo, "Emerging biomedical applications of enzyme-like catalytic nanomaterials," Trends in Biotechnology, vol. 36, no. 1, pp. 15-29, 2018. 
[343] H. Zhang, A. Qu, S. Li et al., "Engineering of chiral nanomaterials for biomimetic catalysis," Chemical Science, vol. 11, 2020.

[344] M.-R. Gao, Y.-R. Zheng, J. Jiang, and S.-H. Yu, "Pyrite-type nanomaterials for advanced electrocatalysis," Accounts of Chemical Research, vol. 50, no. 9, pp. 2194-2204, 2017.

[345] Z. H. Wang, X. Zhang, Y. Q. Zhu et al., "Development of catalyst-sorbents for simultaneous removal of $\mathrm{SO}_{2}$ from flue gas by low temperature ozone oxidation," Ozone: Science \& Engineering, vol. 34, no. 3, pp. 204-212, 2012.

[346] M. Mokhtar, T. S. Saleh, N. S. Ahmed, S. A. Al-Thabaiti, and R. A. Al-Shareef, "An eco-friendly N-sulfonylation of amines using stable and reusable $\mathrm{Zn}$-Al-hydrotalcite solid base catalyst under ultrasound irradiation," Ultrasonics Sonochemistry, vol. 18, 2011.

[347] K. Virmani, C. Deepak, S. Sharma, U. Chadha, and S. K. Selvaraj, "Nanomaterials for automotive outer panel components: a review," The European Physical Journal Plus, vol. 136, no. 9, pp. 1-29, 2021.

[348] S. K. Selvaraj, R. Ramesh, T. Narendhra et al., "New developments in carbon-based nanomaterials for automotive brake pad applications and future challenges," Journal of Nanomaterials, vol. 2021, Article ID 6787435, 24 pages, 2021.

[349] K. Kulkarni, U. Chadha, S. Yadav et al., "Latest trends and advancement in porous carbon for biowaste organization and utilization," ECS Journal of Solid State Science and Technology, 2021.

[350] U. Chadha, P. Bhardwaj, S. Padmanaban et al., "Carbon electrodes in magnesium sulphur batteries: performance comparison of electrodes and future directions," Journal of the Electrochemical Society, vol. 168, Article ID 120555, 2021.

[351] U. Chadha, P. Bhardwaj, S. Padmanaban et al., "Contemporary progresses in carbon-based electrode material in Li-S batteries," Journal of the Electrochemical Society, 2022, In Press. 